NBSIR 76-980

\title{
NBS Space Processing Research
}

E. Passaglia, R. L. |

Institute for Materia National Bureau of : Washington, D. C. : Phone: (301) 921-:

February 1976

Annual Report

For the Period 1 November 1974 - 31 December 1975

NASA Contract W-13,475, Mod. 4

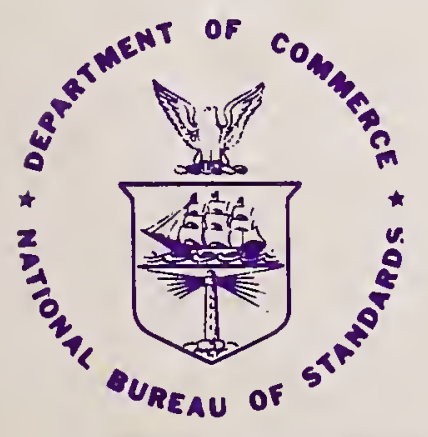

U.S. DEPARTMENT OF COMMERCE

NATIONAL BUREAU OF STANDARDS 



\section{NBSIR 76}

\section{$\underline{\text { NBS } S_{\mid}}$}

E. Passaglia, R. L. Parker

Institute for Materials Research

National Bureau of Standards

Washington, D. C. 20234

Phone: (301) 921-2811

February 1976

Annual Report

For the Period 1 November 1974 - 31 December 1975

NASA Contract W-13,475, Mod. 4

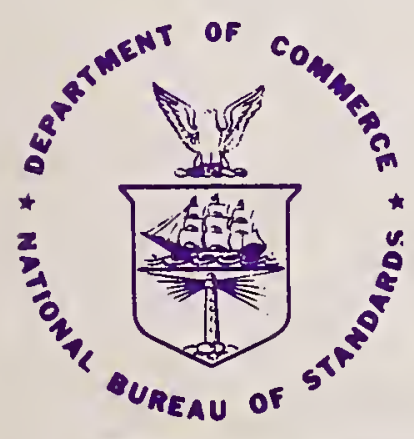

U.S. DEPARTMENT OF COMMERCE

NATIONAL BUREAU OF STANDARDS 



\section{NBS SPACE PROCESSING RESEARCH}

E. Passaglia, R. L. Parker

Institute for Materials Research

National Bureau of Standards

Washington, D. C. 20234

Phone: (301) 921-2811

February 1976

Annual Report

For the Period 1 November 1974 - 31 December 1975

NASA Contract W-13,475, Mod. 4

This report was prepared by the National Bureau of Standards under Contract W-13,475, Mod 4 - NBS Space Processing Research, for the Advanced Missions Program of the National Aeronautics and Space Administration.

U.S. DEPARTMENT OF COMMERCE, Elliot L. Richardson, Secretary James A. Baker, III, Under Secretary

Dr. Betsy Ancker-Johnson, Assistant Secretary for Science and Technology

NATIONAL BUREAU OF STANDARDS, Ernest Ambler, Acting Director 


$$
=-1=
$$


TABLE OF CONTENTS

$\underline{\text { Page }}$

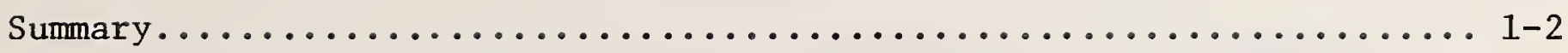

Task 1 - CRYSTAL PERFECTION IN CZOCHRALSKI GROWTH by M. Kuriyama, W. J. Boettinger and H. E. Burdette......... 3-2.j

Task 2 - EVAPORATIVE PURIFICATION OF ULTRA-HIGH PURITY MATERIALS by R. C. Paule, W. J. Boettinger and F. S. Biancaniello...... 22-55

Task 3 - VACUUM EFFECTS IN THE PREPARATION OF COMPOSITE MATERIALS by H. Yakowitz........................... 56-98

Task 4 - MELT SHAPE IN WEIGHTLESS CRYSTAL GROWTH by S. R. Corlell, S. C. Hardy and M. R. Cordes............. 99-119

Task 5 - VAPOR TRANSPORT SYNTHESIS AND CRYSTAL GROWTH by H. S. Parker............................. 120 130

Task 6 - SURFACE TRACTION AND OTHER SURFACE PHENOMENA by A. L. Dragoo............................... 131-141

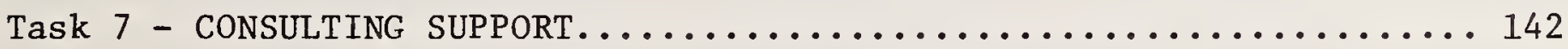

Distribution List.................................... 143-144 



\section{Summa ry}

This report describes NBS work for NASA in support of NASA's Space Processing Program covering the period November 1,1974 to December 31 , 1975. The objectives of the NBS program are to perform ground-based studies (and, where appropriate, space-based studies) of those aspects of space that could possibly provide a unique environment for making materials more perfect or more pure. The approach taken deals primarily with experimental and theoretical studies of the possible effects of the absence of gravitational forces on those materials preparation processes where the presence of gravity may be important in reducing perfection or purity.

The materials preparation processes studied comprise six tasks in the areas of crystal growth, purification and chemical processing, and the preparation of composites. There is an additional seventh task on consulting support.

The results obtained for each task are given in detailed summaries in the body of the report. Briefly, in Task 1 - Crystal Perfection in Czochralski Growth - sizable nickel single crystals have been grown and sliced and the crystal perfection has been assessed by $x$-ray dynamical diffraction techniques. Some preliminary evidence of the effect of growth parameters on crystal perfection has been obtained. Magnetic domain configurations in the nickel slices have been observed with the x-ray technique. In Task 2 - Evaporative Preparation of Ultra-High Purity Materials - in anticipation of possible evaporative purification experiments with $\mathrm{Al}_{2} \mathrm{O}_{3}$ in space, we have measured the relative 
abundances of the various vapor species from molten $\mathrm{Al}_{2} \mathrm{O}_{3}$ and have studied the effects of non-equilibrium radiative heating of the specimen. We have also determined the purification rate of $\mathrm{Fe}$ impurity in molten levitated Mo. The Fe impurity was rapidly reduced from $50 \mathrm{ppm}$ to $50 \mathrm{ppb}$. In Task 3 - Vaccum Effects in the Preparation of Composite Materials - a simple analytical expression is given of the distribution in depth of electronbeam produced $\mathrm{x}$-rays, and the expression's use to analyze layer structures or film composites of particles embedded in a matrix is given. In Task 4 Melt Shape in Weightless Crystal Growth - experimental and theoretical investigations of the floating zone process of crystal growth and purification have been carried out with particular emphasis on microgravity conditions. Both numerical and analytical techniques have been used, and we have experimentally measured liquid zone shape and stability. In Task 5 - Vapor Transport Synthesis and Crystal Growth - seeded growth experiments were undertaken and improvements in the transport tube geometry and technique are described. Growth of mercurous chloride at temperatures in the $350-390^{\circ} \mathrm{C}$ range has resulted in good quality growth of 0.6 O.D. $\times 1 \mathrm{~cm}$ boules. In Task 6 - Surface Traction and Other Surface Phenomena - ground-based experiments and apparatus were developed for studies of convection in drops, including necessary photographic techniques. In Task 7 - Consulting Support - we have continued our rather wide range of meetings, consultations and talks with NASA and NASA contractors. 
Crystal Perfection in Czochralski Growth

M. Kuriyama, W. J. Boettinger and H. E. Burdette

Metallurgy Division

Institute for Materials Research

\section{Summary}

As a continuation and expansion of our previous work (NASA Contract W-13,475 非, Task 1), nickel single crystal growth has been chosen for a study of the relationship between crystal growth zonditions and resultant crystalline perfection in melt-grown crystals. Several interesting crystallographic effects have been found in the resultant crystalline perfection of copper single crystals studied previously. However, the previous work alone is not sufficient to draw the conclusion that the observed crystallographic effects take place during growth of most metal crystals. Nickel has a face-centered cubic crystal structure like copper, while the material properties such as dislocation mobility, stacking fault energy and dislocation velocities are considerably different from copper. In addition, nickel is ferromagnetic. Magnetic domain configurations in the interior of sizable highly perfect nickel single crystals have not been observed before. Possible observation of these domain configurations would be extremely interesting, not only from an academic point of view, but also from an industrial point of view. No one has previously succeeded in producing sizable, highly perfect nickel single crystals. No one has established proper procedures of handling grown crystals chemically and mechanically without destroying the crystalline perfection in the as-grown state. Therefore, emphasis in the present work 
is placed on developing the most suitable technique for the preparation of nickel single crystals. This report should be considered to be a preliminary report to more systematic and complete future investigations.

In this report, the most suitable chemical processing method has been established experimentally. These methods will enable us to seek the relationship between crystal growth conditions and resultant crystalline perfection systematically with reasonable reproducibility in future work. The crystal perfection has been assessed by non-destructive methods of $x$-ray dynamical diffraction as in the previous work. Preliminary trends of data have indicated that the growth condition parameters seem to affect the resultant crystalline perfection. This observation is discussed in comparison with the previous work on copper, although it would be premature to form any conclusion. The entire procedures have not yet become routine, nor perfect.

A remarkable achievement of the present work is the observation of magnetic domain arrangements in the interior of nickel single crystals. If one can systematically produce nickel single crystals which would be better in perfection than those obtained in this report, one can study the magnetic properties related to magnetic domain motions in an idealized condition in order to improve electromagnetic performance for possible industrial purposes.

In view of the progress made in this preliminary study, it is strongly recommended that this study should be continued in an effort to find whether an optimum choice of growth conditions improves the resultant crystal perfection, and, if so, to evaluate the role of space in producing nickel single crystals of better perfection. 


\section{Introduction}

Space has provided mankind with another dimension, which future industries may find unique and useful for the production of new materials. The lack of gravity and the availability of a high vacuum will be significant factors in the progress of technology in many areas. It is learned from experiences in the past that new technology produces new materials, such as silicon or germanium single crystals free of crystal imperfections. These single crystals have shown their great usefulness in semiconductor industries through the developments of communication devices: computer elements, visual display elements and many other products.

Although it may not be recognized explicitly, the usefulness of single crystals or textured materials has been demonstrated, for example, through the use of Fe-Si alloys for increased performance of transformers and motors. In the days of energy conservation, this aspect may have great significance. No matter what methods will be used to produce energy in the future, it will very likely be distributed to the users in the form of electricity. In this sense, the production of new materials of superior performance should be sought in all possible ways.

At present, Fe-Si alloys are unique, because of their desirable performance in cyclic magnetization processes, their easiness of being rolled in a commercial size and their rather good level of crystal perfection. In contrast, most other fundamental ferromagnetic materials have not so far satisfled high performance conditions for many reasons. For example, no one has succeeded in producing sizable, highly perfect nickel single crystals, which might be the precursor of commercial textures materials. These perfect magnetic single crystals provide an idealized condition for a study of 
magnetic domain behavior, which will assist in gaining control over magnetic properties under cyclic magnetization processes for possible industrial applications .

It has been established in previous reports* that crystal growth conditions play a significant role in producing sizable copper single crystals of high perfection from the melt by the Czochralski technique. The crystal growth conditions have been described in terms of directly controllable process parameters, such as the rotational speed of the seed crystal and of the melt. These parameters represent the fluid flow conditions in the melt during crystal growth. As discussed in Report III, the reproducible relationship between the crystal growth conditions and the resultant crystalline perfection has considerable practical significance in space manufacturing processes, since the lack of gravity and, in turn, the lack of thermal convection may make the resultant fluid flow in the melt more controllable. It has been concluded further in Report III that, although an optimum growth condition for the preparation of highly perfect copper crystals is, as a rule, characterized by a physical quantity controlling the fluid flow in the melt, more crucial factors influencing the ultimate level of crystal perfection are found in some crystallographic effects during growth: the growth direction and the formation of a particular set of sessile dislocations. It is worthwhile mentioning that this second factor may be related directly to the effect of gravity. Are these crystallographic effects more prominent or more likely to happen in copper single crystals than in other crystals?

* The previous reports will, hereafter, be referred to as Report I (Government Order H-84832A, National Aeronautics and Space Administration, NBS report 10873), Report II (Contract W-13,475 No. 1 - NBS Materials Science and Manufacturing in Space Research for the Advanced Missions Program of the National Aeronautics and Space Administration; NBS report NBSIR 73-402) and Report III (Contract W-13,475 Mod. 3 - NBS Materials Science and Manufacturing in Space Research for the Advanced Missions Program of the National Aeronautics and Space Administration; NBSIR 74-611). 
The previous work lacks evidence on this aspect. While nickel also has a face-centered cubic crystal structure, its material properties such as dislocation mobility, stacking fault energy and dislocation velocities are considerably different from copper. In view of these crystallographic, mechanical and magnetic properties, we have chosen nickel single crystals in the present work to study the relationship between the crystal growth conditions and the resultant crystal perfection. Since there is, at present, not much previous information available for the growth of sizable, highly perfect nickel crystals, emphasis in the present report is placed on developing the most suitable technique for the preparation of nickel crystals. This report is, therefore, considered to be a preliminary report to more systematic and complete future investigations.

\section{Experimental Procedures}

As described in previous reports, a vital part of research of this type is the non-destructive assessment of crystal perfection in the interior of crystals by dynamical diffraction. The anomalous Borrmann transmission effect serves, to lowest degree, as a measure indicating whether crystals are of a sufficient quality to be investigated further. When a crystal is imperfect, ordinary $\mathrm{x}$-rays cannot penetrate farther than $10 \mu \mathrm{m}$ of the crystal even when a Bragg diffraction condition is met. On the other hand, these $x$-rays can pass through a crystal one millimeter thick under Bragg diffraction conditions (the anomalous transmission effect), when the crystal attains a certain level of perfection. Above this level of perfection, crystals begin to display their special characteristics unobtainable in imperfect crystals or polycrystalline materials. It is, therefore, important to find a method which is capable of producing crystals above this level, and to establish a method of characterizing the crystal perfection without further damaging the crystals in the evaluation processes. 
There have been two reports ${ }^{1,2}$ in the past, of which we are aware, of $x$-ray topographic observation of nickel crystals. One ${ }^{1}$ dealt with nickel crystals in plate form prepared from nickel bromide by chemical reduction, and the other ${ }^{2}$ with nickel sheets treated by a strain anneal (secondary crystallization) method. The first group obtained diffraction topographs in the transmission geometry from large crystal grains in the nickel plate. However, the crystal was very thin (in addition, with a hard radiation). Consequently, the anomalous transmission effect was not prominent. The latter used a multi-grain sample $0.5 \mathrm{~mm}$ thick, and observed the anomalous transmission effect in one of the grains. However, there have been no systematic studies on the production of nickel crystals of high perfectior. In particular, no one has yet succeeded in growing sizable nickel single crystals of high perfection from the melt.

In view of this, particular efforts were placed during this contract year on the following aspects:

(I) development of a method of growing nickel crystals from the melt by the Czochralski technique, and

(II) development of chemical processing methods for obtaining samples from grown crystal boules for $x$-ray topographic examination.

Since we had not anticipated obtaining crystals of good quality at the onset of this work, an additional effort was made on

(III) modification of the asymmetric (double) crystal topographic (ACT) camera for such imperfect crystals.

\section{Crystal Growth}

One of the objectives for the present contract year is to grow sizable nickel single crystals by the Czochralski method using the same equipment used for the previous copper work. A considerable amount of time has been 
spent to achieve this objective with limited resources. First, the entire furnace and vacuum system was completely disassembled, so that the system could be cleaned chemically to remove all deposits and traces of copper. Wherever possible, parts were etched with various acid solutions. The system was then reassembled with new seals, and all the parts contaminated heavily by copper were replaced with new ones up to the limit of commercial. availability.

It was found, after testing the system, that the melting point of nickel was at the extreme limit of the furnace capability and that the system could not hold a good vacuum at this high temperature. These facts necessitated some modifications in thermal shields and for a supply of various atmospheres. Several atmospheres were tested, but all of them accentuated heat loss. Finally, it was concluded that an Ar gas could be tolerated under the present conditions because of its less efficient heat transfer property than other inert gases.

It is extremely desirable to maintain visual contact with the meniscus area during crystal growth. This condition imposed severe constraints on the arrangements of thermal shields and on the choices of crucible shapes. Several crucible shapes of different materials were tested, including the dual crucible combination utilizing a graphite outer crucible. Cylindrical alumina crucibles have been selected as the most convenient at present. After each use, the crucible was discarded to avoid possible cracking during subsequent crystal growth.

For this year's purpose, $99.99 \%$ commercially available nickel stock was obtained in the form of $6 \mathrm{~mm}$ diameter rods. We have not yet been concerned with the purity of nickel in the present work which is still at the preliminary stage of establishing a proper procedure of nickel single crystal growth. 
Large nickel crystals were grown with this arrangement in a fashion similar to that used previously for copper single crystal growth. Either a polycrystalline or a single crystal seed was used. Both the seed and the melt were rotated around a common axis independently with different rotational speeds. The pulling speed was chosen to be $0.10 \mathrm{~cm} \mathrm{~min}^{-1}$ for convenience. During the crystal growth, attempts were always made to narrow the diameter of a crystal boule initially at one region (bottlenecking). Bottle-necking was not successful most of the time, because of the lack of temperature control, as described later. The grown crystals were about $12 \mathrm{~cm}$ long with diameters between 1.5 and $2.5 \mathrm{~cm}$. When crystals were bottlenecked successfully, the neck diameter was always slightly less than $1 \mathrm{~mm}$. On removing the grown boules with a bottleneck from the furnace, the waxing technique was used as in the copper work described in Report II.

\section{Crystal Cutting and Polishing}

For the assessment of crystal perfection it is necessary to prepare smooth single crystal surfaces of desired crystallographic orientation without introducing further damage into the crystals during the preparation. It is usually desirable to obtain samples of different crystallographic orientation in a disc form from the same grown boule. The conventional methods of cutting and polishing introduce dislocations to a considerable depth below the crystal surface. There have been two reports ${ }^{3,4}$ in the past concerning the chemical cutting and polishing of nickel crystals. However, these reported techniques were found to be unsatisfactory when the crystal perfection in original boules was improved. During this contract year, extensive work has been carried out to develop the chemical procedures for cutting and polishing nickel single crystals. 
We used the same cutter which was developed for copper single crystals in Report III. The following chemical compositions were found to be most effective for the strain-free cutting of samples from the grown nickel boules:

$\begin{array}{lr}\text { nitric acid } & 45 \text { volume \% } \\ \text { hydrochloric acid } & 33 \text { volume \% } \\ \text { acetic acid } & 20 \text { volume \% } \\ \text { ethyl alcohol } & 2 \text { volume \% }\end{array}$

Among these, ethyl alcohol plays an important role in maintaining the solution in a highly volatile state. $\Lambda$ freshly prepared solution should be fed into the cutting baths (acid trays) at regular intervals. It is also found to be necessary to heat the crystal boule to about $90^{\circ} \mathrm{C}$ in order to keep the cutting time reasonable. An infrared heat lamp with an aperture was used to heat the boule so that heating could be concentrated in the cutting areas to avoid softening of the glue which was used to hold the boule in a desired orientation.

This new highly volatile cutting solution and the addition of heat made it necessary to establish a new way of mounting the oriented boules without straining them. A silicone-rubber compound was found to withstand the conditions and was easily removed afterwards. It rapidly became obvious that any solution which would cut nickel effectively would also attack stainless steel, plastic and most other materials. This necessitated several modifications to the acid saw which was described in Report III. The motor was removed to a remote location and a drive train was installed. The stainless steel rollers in the acid trays were replaced with precision-made quartz rollers. A high grade tungsten wire was found to perform adequately for reasonable amounts of time in carrying acid solutions. Since our acid saw was made of stainless steel, a sprinkler system was installed to continuously wash the cutting solution and reaction products off the saw to prevent the saw from corroding. 
After the boule was sliced, samples should be chemically polished to obtain smooth surfaces without introducing dislocations in the samples. After various solutions had been examined under a variety of conditions, an optimum polishing solution and procedure were developed. The efficient strain-free polishing can be best achieved when the samples were polished with different solutions at several stages. The basic recipe consists of nitric, hydrochloric, acetic acids and ethyl alcohol. The compositions should be changed at each stage; ethyl alcohol again plays an active role in making the solutions volatile. The solutions should be prepared shortly before use. Because of the volatile nature of the solutions, the polisher previously described in Reports I and II had to be modified. The present polisher has several replacement parts made from teflon to enable it to withstand such vigorous polishing solutions.

Besides the new developments described above, the other procedures are almost the same as used for the previous copper work (Reports I and II). The boules are mounted on a goniometer stage to be oriented in desired crystallographic directions and are transferred to the acid saw which can accommodate the goniometer stage in precisely the same alignment. The slices are handled very carefully and mounted on bakelite cylinders with the same glue as developed for copper crystals for acid polishing. The sample discs are later removed from the cylinders by dissolving the glue and mounted on stainless steel rings which can attach to a goniometer head for x-ray topographic examination.

\section{Dynamical Diffraction Topography}

For the non-destructive assessment of the perfection of sample crystals, two different $\mathrm{x}$-ray optical arrangements were employed as in the previous copper work, namely an asymmetric (double) crystal topographic (ACT) camera 
and a high-resolution double-crystal scanning diffractometer (SCAD). The details of these systems have been described in Reports I, II and III and also in previous papers 5,6 . An $x$-ray image intensifying tube has been used as described in Report III.

At the onset of this work, a rocking device was added to the ACT camera to change the $\mathrm{x}$-ray glancing angle continuously during photographic exposure. Until one finds an optimum growth condition and chemical preparation methods, the perfection of grown crystals are usually anticipated to vary over a large range, possibly including a polycrystalline or multi-grained state. This rocking device enables us to obtain topographs from such imperfect crystals. From a view of x-ray diffraction optics, this device allows us to lower the optical quality of our ACT system essentially down to the Berg-Barrett method.

The thicknesses of the nickel discs were determined by the anomalous transmission effect in the same fashion as described in Reports I, II and III and previous papers 5,6 for copper crystals. The SCAD system was used to obtain the profiles and widths of rocking curves at various locations in the crystal. CuK $\alpha$ radiation was used throughout the present work. The product of the ordinary linear absorption coefficient, $\mu=43.9 \mathrm{~mm}^{-1}$, and thickness, L, ranged from 10 to 45 in the present work. This product plays an important role in dynamical diffraction to indicate the degree of crystal perfection in the interior of crystals. The higher the value of $\mu \mathrm{L}$ for which anomalous transmission is observed, the better the crystal perfection.

X-ray diffraction topographs were taken both in the surface reflection and in the transmission geometry with those two systems. The sample discs were prepared in the $\langle 110\rangle$ orientation to supply a maximum number of different lower index symmetric reflections; $2 \overline{2} 0$ surface reflection, $11 \overline{1}, 111$, 
002 and 220 transmission topographs can be obtained from the same nickel discs.

As mentioned before, the main efforts were concentrated on the development of the techniques. These are indispensable steps before the ralationship between the crystal growth conditions and the resultant crystal perfection is sought systematically with reasonable reproducibility. We undertook the present work as a preliminary step to the establishment of a systematic procedure to achieve our final goal in the future. In the present work, we therefore arranged various factors carefully to perform 24 nickel crystal growth experiments. All the stages of the nickel growth and preparations have taken much longer time than the previous copper procedures. For example, the pre-baking of crucibles and extensive preparation of the charge have created extra work which was not necessary for the copper work. The cutting and polishing are much slower with nickel than copper. This fact, combined with the highly volatile and corrosive nature of the solutions and their effect on equipment, has severely limited the quantity of samples for a given period.

\section{Experimental Results}

Large nickel single crystals were grown by the Czochralski technique from either a polycrystalline or a [100] oriented single crystal seed. The crystal boules grown with a polycrystalline seed are specified by the number 0 in the third digit of their sample number, for example, 170200, and the boules grown from the [100] oriented seed by the digit 4. The melt and seed were rotated in the same direction with approximate speeds of $10 \mathrm{r} . \mathrm{p} . \mathrm{m}$. ; the relative speed of rotation ranged from 0.2 to $0.6 \mathrm{r.p} . \mathrm{m}$. This growth con- 
dition is denoted by condition 2, which appears in the fourth digit of the sample number.

The growth directions of the crystal boules grown with a polycrystalline seed have been determined by the Laue method as shown in Fig. 1. The growth direction of the boules grown with the oriented single crystal seed has been confirmed to be the [100] direction, which is the crystallographic orientation of the seed. As described previously, the bottlenecking was not always achieved. An example of the boule shape with a successful bottleneck is shown in Fig. 2. The boules whose sample numbers are less than 120200 were exhausted during the process of collecting the necessary information for the development of nickel crystal growth method and chemical processing methods described in a previous section.

Fig. 3 shows a $2 \overline{2} 0$ diffraction topograph of nickel sample $\#_{12} 20223$ taken with the ACT in the surface reflection geometry. This sample was sliced out of boule \#120200 which was grown without a successful bottleneck. During the growth, the diameter along the length of the boule was not controlled satisfactorily. This topograph indicates that the sample consists of many small grains, whose orientations spread out over more than several minutes of arc. This sample should not be considered a single crystal. Some sample discs show a distribution of much larger subgrains in their surface reflection topographs as shown in Fig. 4, when they are sliced out of boules grown under a similar poor diameter control condition but with a reasonable bottleneck. No anomalous transmission was observed in sample crystals whose surface reflection topographs were as poor as those in Figs. 3 and 4. This observation was made without exception, in particular, for the boules of the 0-series grown from a polycrystalline seed. This is not true for sample crystals of the 4 series, as shown later. 
In some occasions crystal boules were grown with a narrow bottleneck and reasonable diameter control was achieved over the entire length. Surface reflection topographs of sample discs from these boules display a drastic improvement in their crystal perfection. The topographic images cover the entire exposed area of the sample discs, as shown in Figs. 5 and

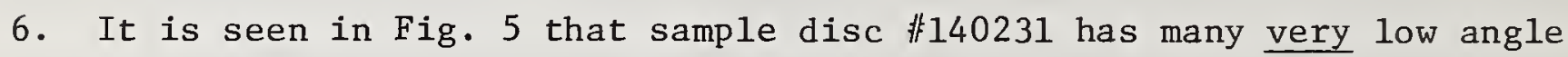
subgrain boundaries, while sample 170231 has very few of them as shown in Fig. 6. In the ACT system, the misorientations of these subgrains are judged by the image coverage to be much smaller than 30 seconds of arc. Horizontal line images in Fig. 6 correspond to scratches on the surface, which were created in handling. Crystals grown in conditions similar to those used for these two crystals produced surface topographs almost as good as (or even better than) those shown in Fig. 5 and 6 . All of these crystals demonstrate anomalous transmission from their entire volume, as shown, for example, in Fig. 7.

Fig. 7 shows $\overline{1} \bar{l} 1$ transmission topographs taken with the ACT in the as-grown state of a sample crystal $0.9 \mathrm{~mm}$ thick, \#140231. A topograph of

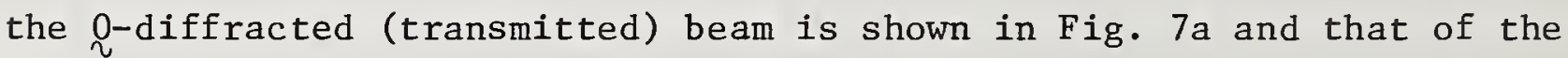
$\underset{\sim}{H}$-diffracted (Bragg-diffracted) beam is shown in Fig. 7b. A lighter (white) area indicates that more $\mathrm{x}$-ray photons have reached this area, while a dark (black) area implies that photons have been disrupted by the crystal. Most of the details in both topographs $a$ and $b$ are identical; in particular, the images caused by the presence of crystal imperfections such as dislocations, dislocation clusters and very small angle subgrain boundaries match perfectly with each other. It should be noted that the topographs have revealed the entire shape of the crystal disc, indicating that the prominent anomalous transmission effect has taken place all over the crystal. Fig. 8 a and b 
show $11 \overline{1}$ transmission topographs of the $\ell$-diffracted and the $H$-diffracted beams taken with the ACT system in the as-grown state of sample crystal \#170231. This crysta1 is more perfect than 1 140231, as there are fewer images caused by crystal imperfections in these topographs than in Fig. 7.

In addition to the crystal-imperfection images mentioned above, there are extremely straight line images throughout the crystal. Also visible are images of rectangular shape. Most of these straight line and rectangular images are nearly identical except for minor details in both topographs $\mathrm{a}$ and $\mathrm{b}$. However, as shown in the enclosed area A in Fig. 7, some of the images are different in contrast in the two topographs. When the crystal is magnetized by the application of a magnetic field of roughly $2.39 \times 10^{5}$ $\mathrm{A} / \mathrm{m}$ in one of the easy directions of magnetization, [11]], the straight line images and rectangular image arrangements disappear completely, except for one area at the right bottom corner, as shown in Fig. 9 a and b. In such magnetic fields, nickel single crystals are magnetized to a single magnetic domain.

The formation of more complicated patterns is shown in Fig. 10 a and $\mathrm{b}$, when a magnetic field of $1.43 \times 10^{4} \mathrm{~A} / \mathrm{m}$ is applied in one of the hard magnetization directions, [001]. It should be noted that the disruption images due to crystal imperfections have remained unchanged in the application of a magnetic field. After the magnetic field is removed, the straight line and rectangular images recovered as shown in Fig. 11 a and b. Almost all of these lines, including line segments, are parallel to either the [001] or [110] direction. All the topographs give evidence that those straight line and short line-segment images are indeed related to the magnetic domains in the interior of crystals and that these images very likely correspond to magnetic domain walls. A more detailed study 
of the magnetic domain images is presented elsewhere? An example of diffraction topographs taken from the $\underset{\sim}{0}$-diffracted and the $\underset{\sim}{H}$-diffracted beams with the SCAD system is shown in Fig. 12 a and b.

Next, we describe the topographs obtained from the crystals of the 4-series which were grown in the $\langle 100\rangle$ direction. So far we have not succeeded in narrowing the boules initially to a reasonable bottleneck diameter. Diameter control was achieved satisfactorily. An example of ACT $2 \overline{2} 0$ surface reflection topographs is shown in Fig. 13. This topograph indicates that the crystal consists of several large subgrains. Unlike the crystals of the 0 -series, anomalous transmission was obtained from the crystals of the 4-series, even though subgrains exist. An example of an

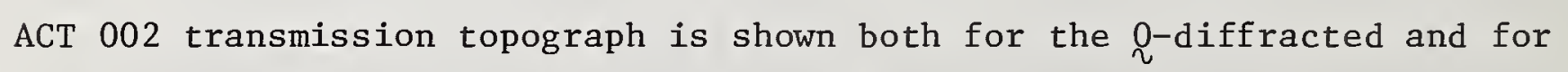
the $\underset{\sim}{H}$-diffracted beam in Fig. 14 a and b. Images caused by magnetic domains are visible, along with many disrupted images caused by crystal imperfections. Usually spectroscopic data - namely, rocking curve profiles and widths - alone would supply adequate information regarding the degree of crystal perfection in sample crystals, as demonstrated previously. However, nickel crystals grown so far are not perfect enough to be judged by their spectroscopic data. Here we only show those data obtained from the sample crystals above a certain level of perfection. The rocking curves were obtained from these sample discs at various locations with the SCAD for the $2 \overline{2} 0$ diffracted beam in the surface reflection geometry and the $11 \overline{1}$ in the transmission geometry. The full width at half maximum (FWHM) of the $2 \overline{2} 0$ rocking curve were 34 seconds of arc and 30 seconds of arc in average for $\# 140231$ and $\# 170231$, respectively, except for an extremely distorted area. The perfection is not uniform throughout 
the crystal. The local variations of rocking curve widths are quite significant. The severely distorted area showed a width up to 64 seconds of arc. An example of the $2 \overline{2} 0$ rocking curve profiles is shown in Fig. 15. For the $11 \overline{1}$ rocking curves in the transmission geometry, the FWHM values were, in the neighborhood of 12 seconds of arc. An example of the profiles is shown in Fig. 16a. The local variation of the profiles is dramatically demonstrated in the transmission geometry as shown in Fig. 16b.

\section{Conclusion and Discussion}

Major emphasis of this work was placed on the development of chemical processing procedures as well as the workable modification of the crystal grower for nickel crystals. In this work we have only begun the first step of the nickel crystal growth program. The entire procedures have not become routine, nor become perfected. In this type of research, it is extremely important to find the reproducibility of results under a given set of conditions. Admittedly, we have not started to collect our data along this desired line. We have even set a pulling speed at an unusually high value so that we can explore all the other possibilities most effectively to approach a final desired condition as quickly as possible. For this reason, this report is merely a preliminary study of the perfection of nickel crystals grown from the melt. The relationship between the growth conditions and the resultant perfection cannot be discussed sensibly in this report. We do not even know a reasonable set of controllable process parameters for the growth at this stage. It is extremely important to continue this effort to obtain optimum growth conditions for highly perfect nickel single crystals before we undertake any growth experiments of nickel crystals in space. 
Even though the present work is preliminary to more systematic investigations, we have obtained some facts which are compatible with those obtained in the previous work of copper. For example, diameter control over the entire length of grown crystals is quite desirable to obtain the crystal perfection above a certain level. Failure of diameter control usually results from poor temperature control which increases the chance of getting more dislocations into crystals through suddenly exposed new crystal surfaces. Another factor which is consistent with the previous results on copper is that bottlenecking is indispensable in obtaining crystals of good quality. The present results indicate that the crystals grown with a narrow neck have demonstrated the anomalous transmission effect. It was concluded in the previous copper work that some crystallographic effects influence the resultant perfection of copper single crystals. We have seen a similar effect on the crystal perfection of nicke1 in the present work, although the present data may not be enough to prove it. The crystals grown in a crystallographic direction, say, [100], have demonstrated the anomalous transmission effect even though the crystals consist of many subgrains whose misorientations are very sma11.

A remarkable achievement of the present work is the observation of magnetic domain arrangements in the interior of nickel single crystals. No one has succeeded previously in observing such magnetic domain arrangements spreading over the entire crystal volume in nickel crystals. The $\mathrm{x}$-ray image formation of magnetic domains in crystals is purely dynamical. The crystals are, therefore, required to be above a certain level of perfection. Fortunately, we have achieved this level of perfection in the present work. Since magnetic domain arrangements are closely related to the crystal perfection in the bulk, it is desirable to find a correlation between the 


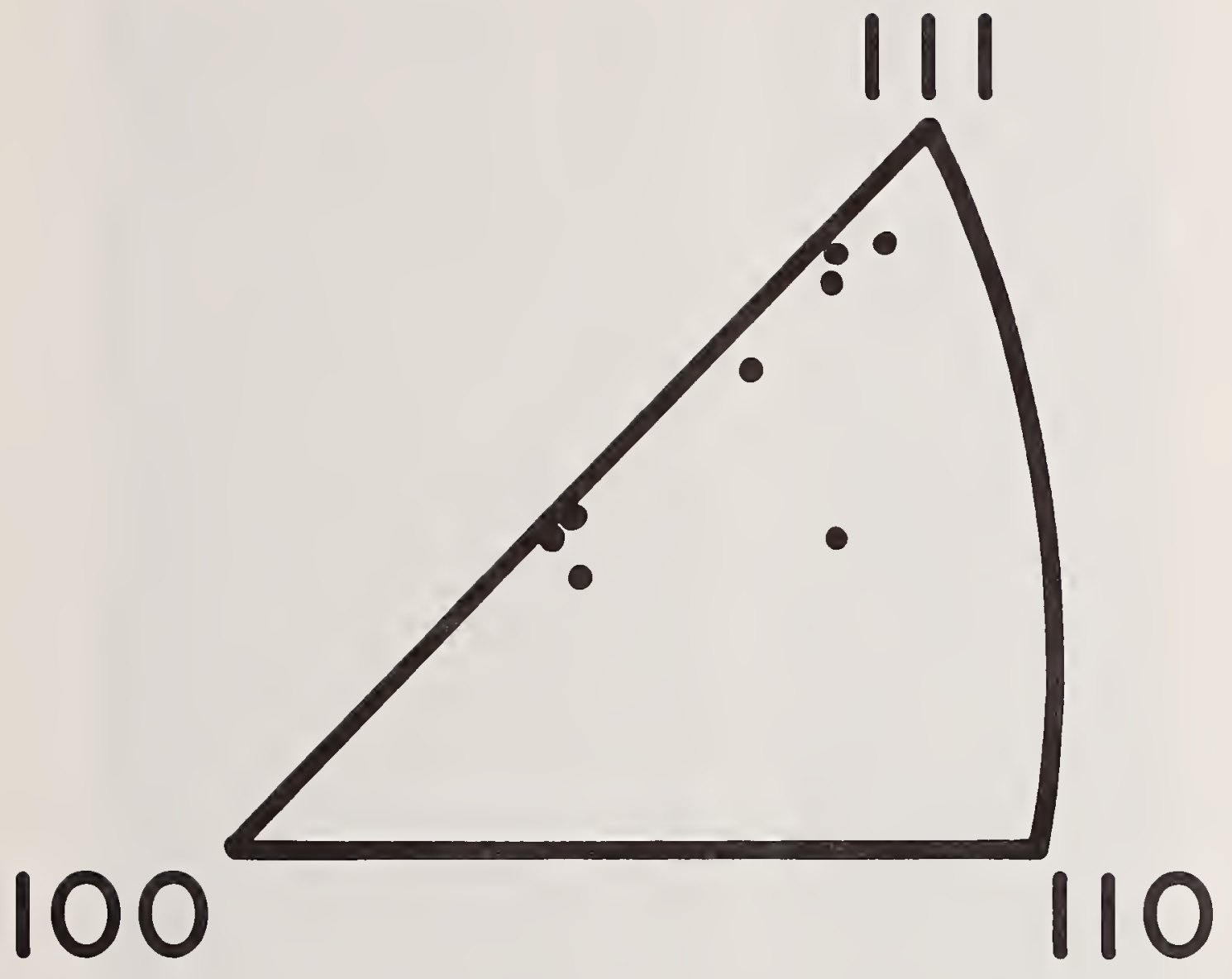

Fig. 1. Stereographic plot of the growth directions of crystal boules grown from polycrystalline seeds by the Czochralski technique. 



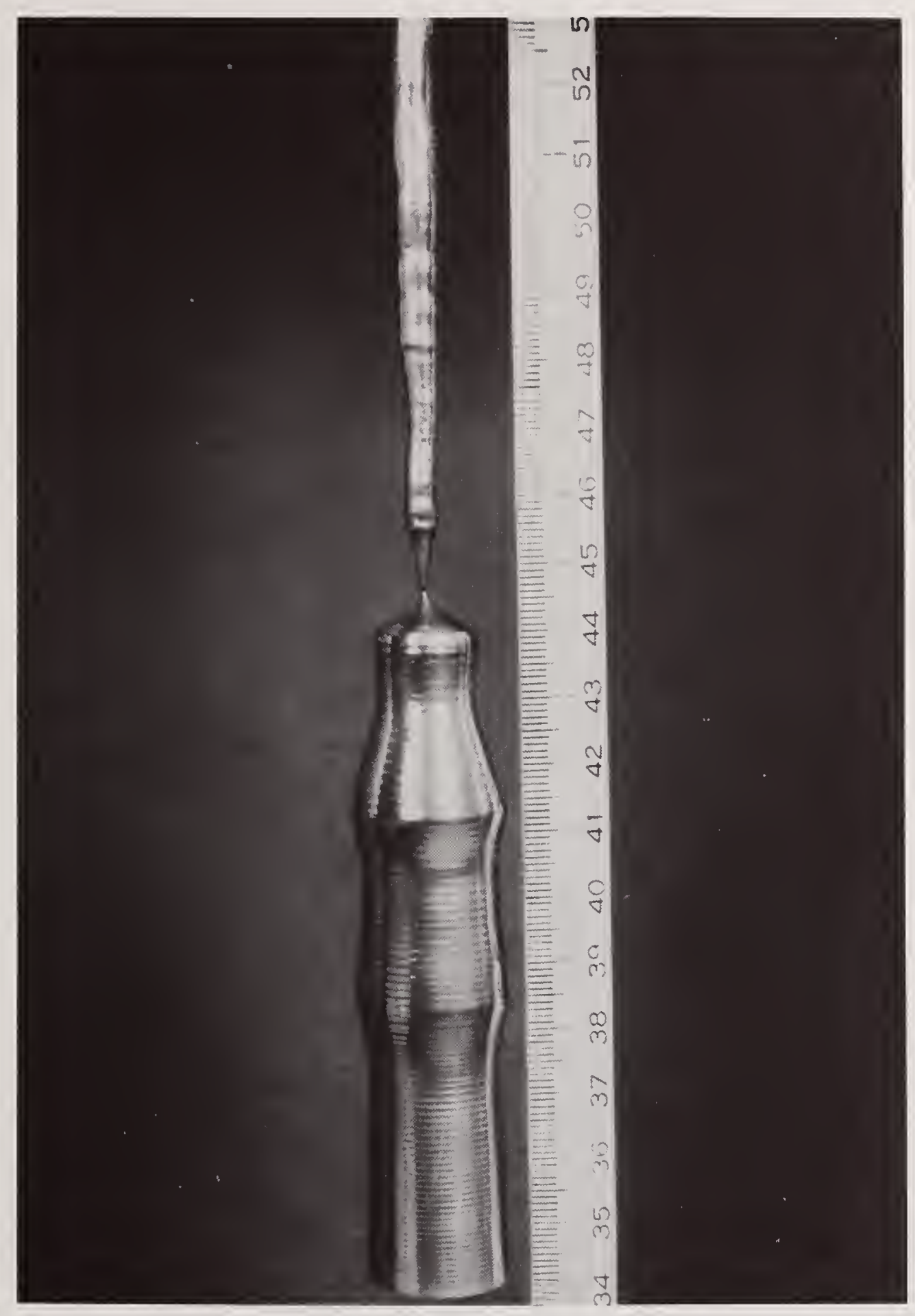

Fig. 2. Photograph of a $\mathrm{Ni}$ single crystal grown by the Czochralski technique with bottleneck diameter roughly $1 \mathrm{~mm}$. 



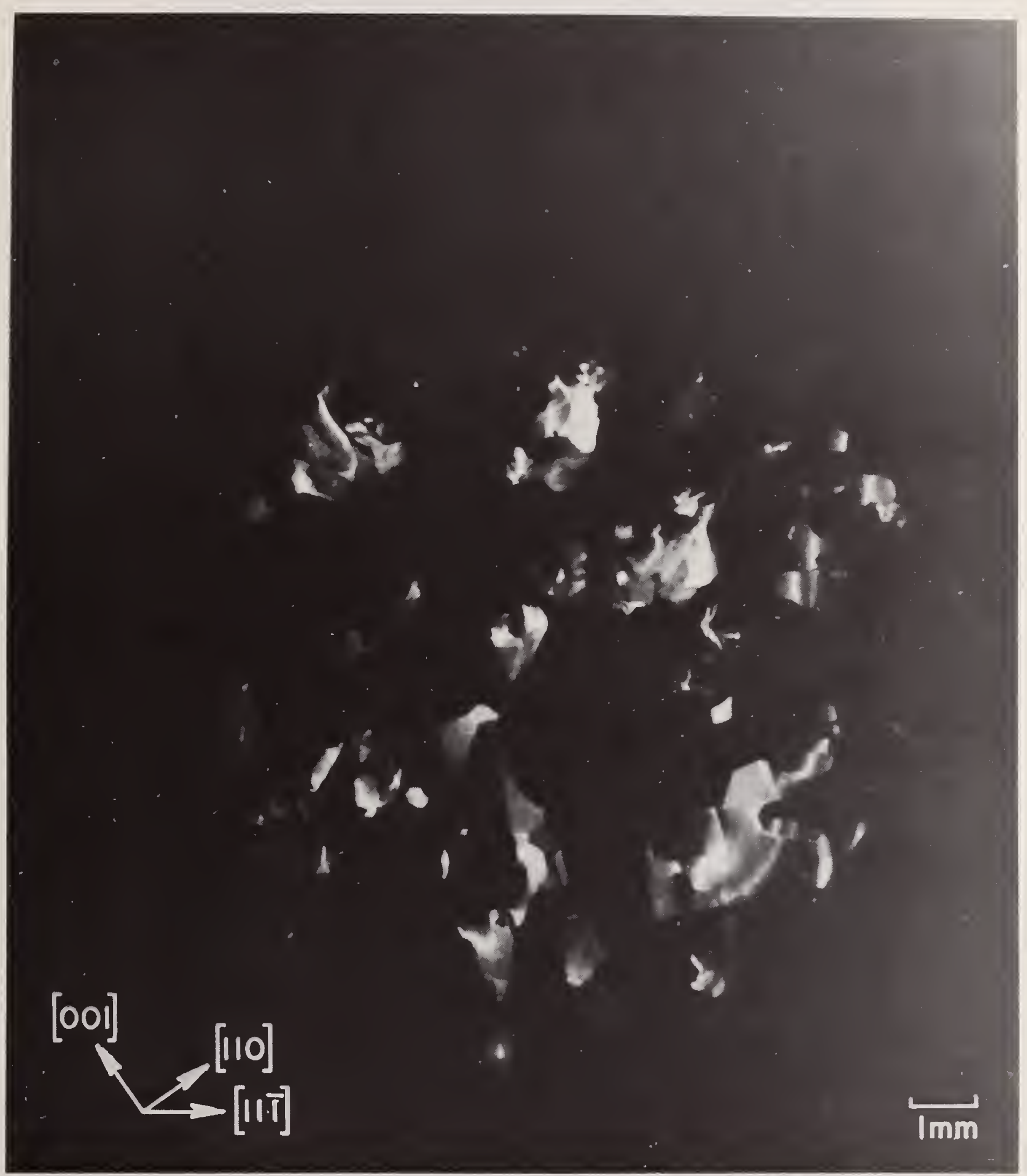

Fig. 3. ACT $2 \overline{2} 0$ surface reflection topograph of a very poor quality as-grown $\mathrm{Ni}$ crystal (非20223) grown without a bottleneck and with poor diameter control. Note the very small subgrains. 



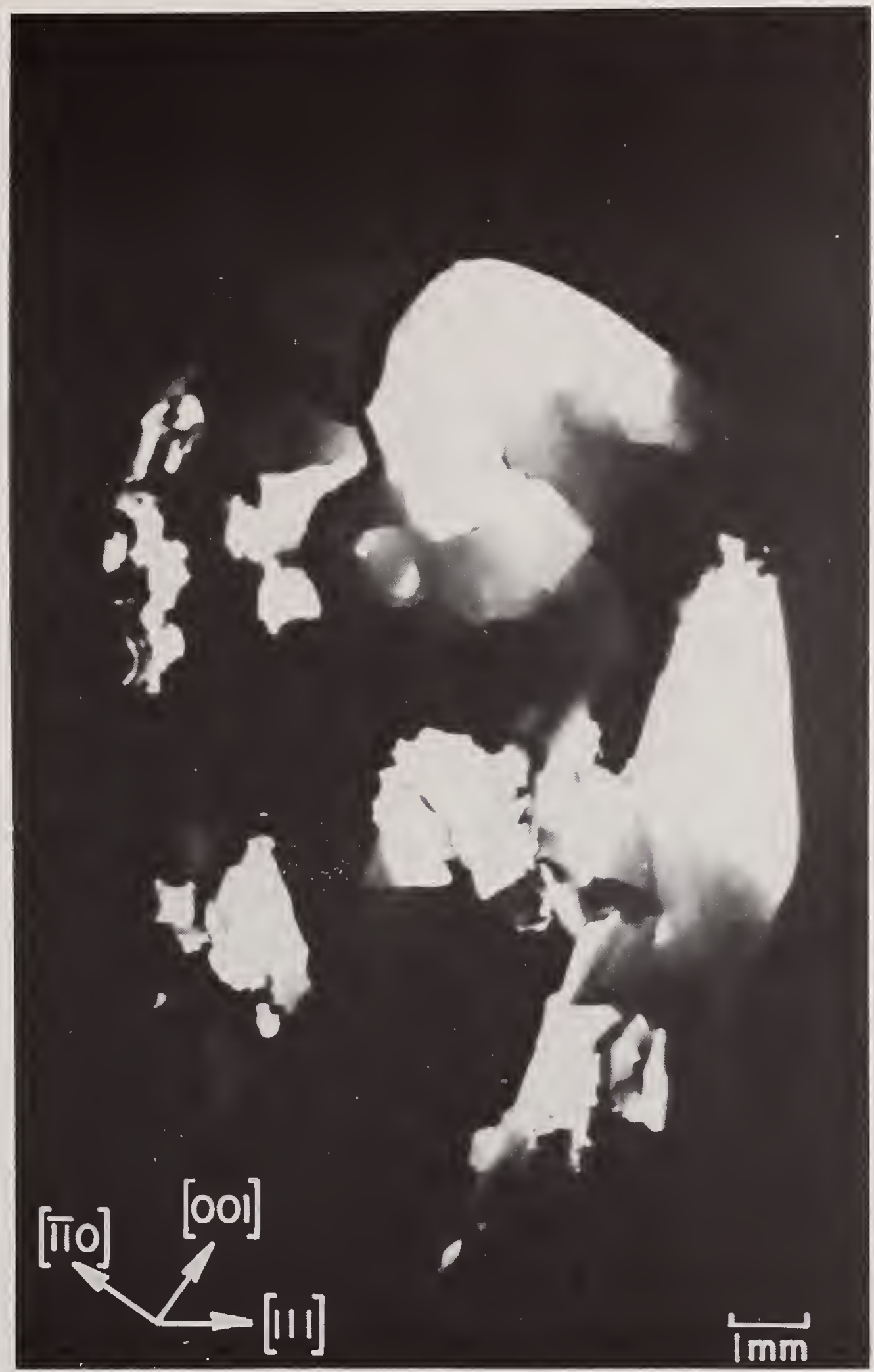

Fig. 4. ACT $2 \overline{2} 0$ surface reflection topograph of as-grown Ni crystal 非130221 grown with a bottleneck and with poor diameter control. Note the large sub-grains. 



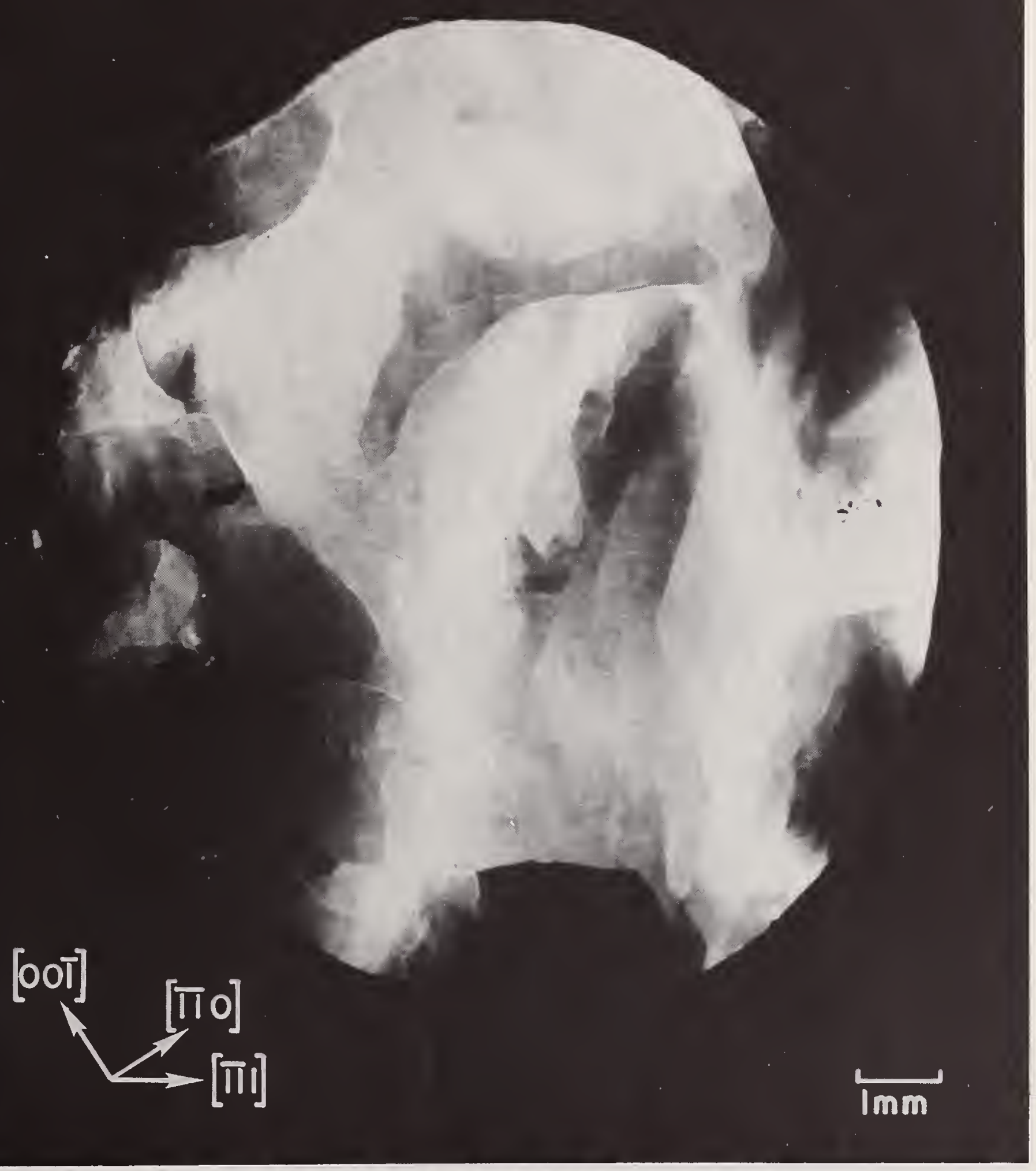

Fig. 5. ACT $\overline{22} 0$ surface reflection topograph of as-grown Ni crystal 非 140231 grown with a bottleneck and good diameter control. Note that diffraction from the entire area of the crystal indicates that misorientation of sub-grains is less than $30 \mathrm{sec}$. of arc. 



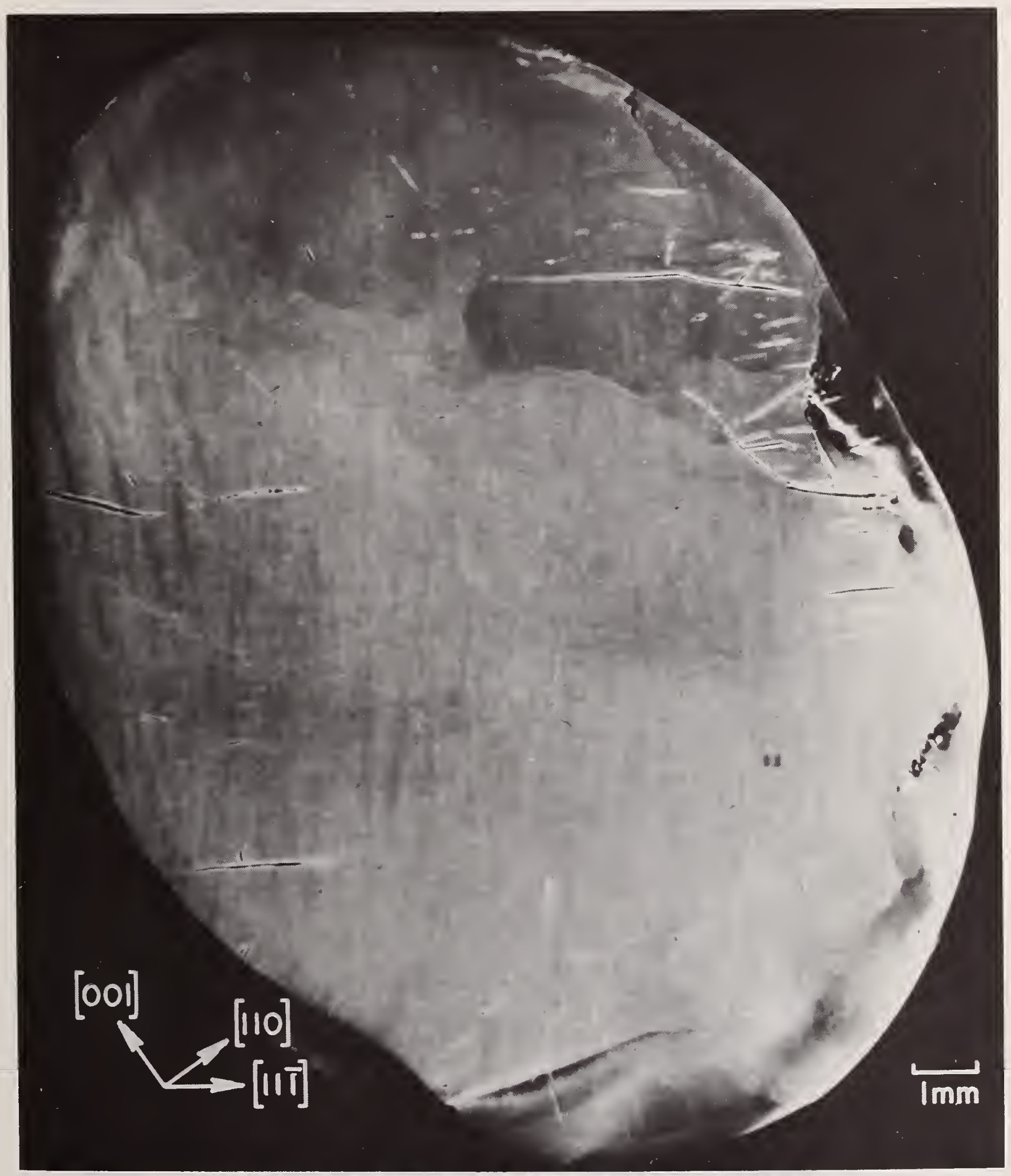

Fig. 6. ACT $2 \overline{2} 0$ surface reflection topograph of a good quality as-grown $\mathrm{Ni}$ crystal (非170231). 



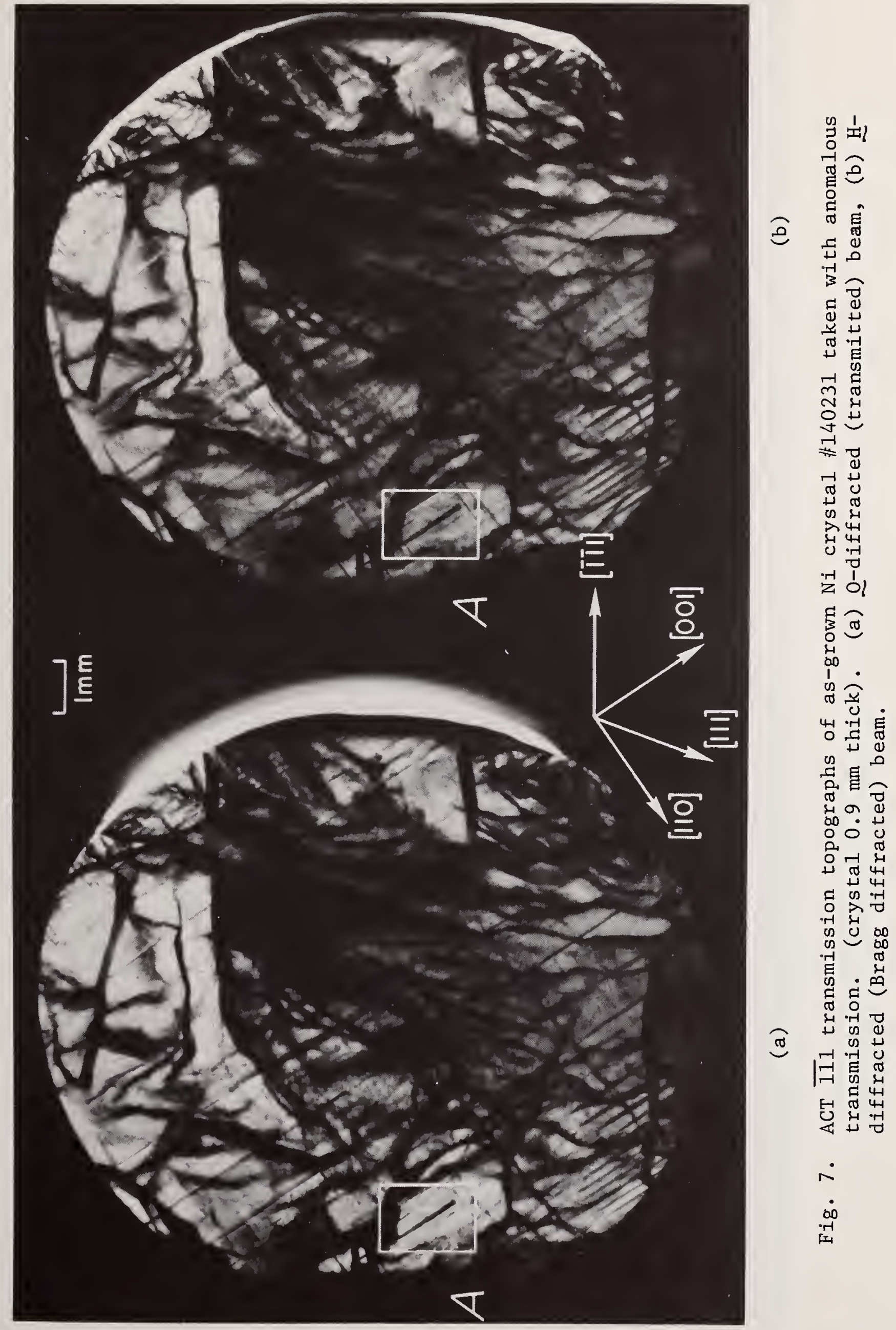





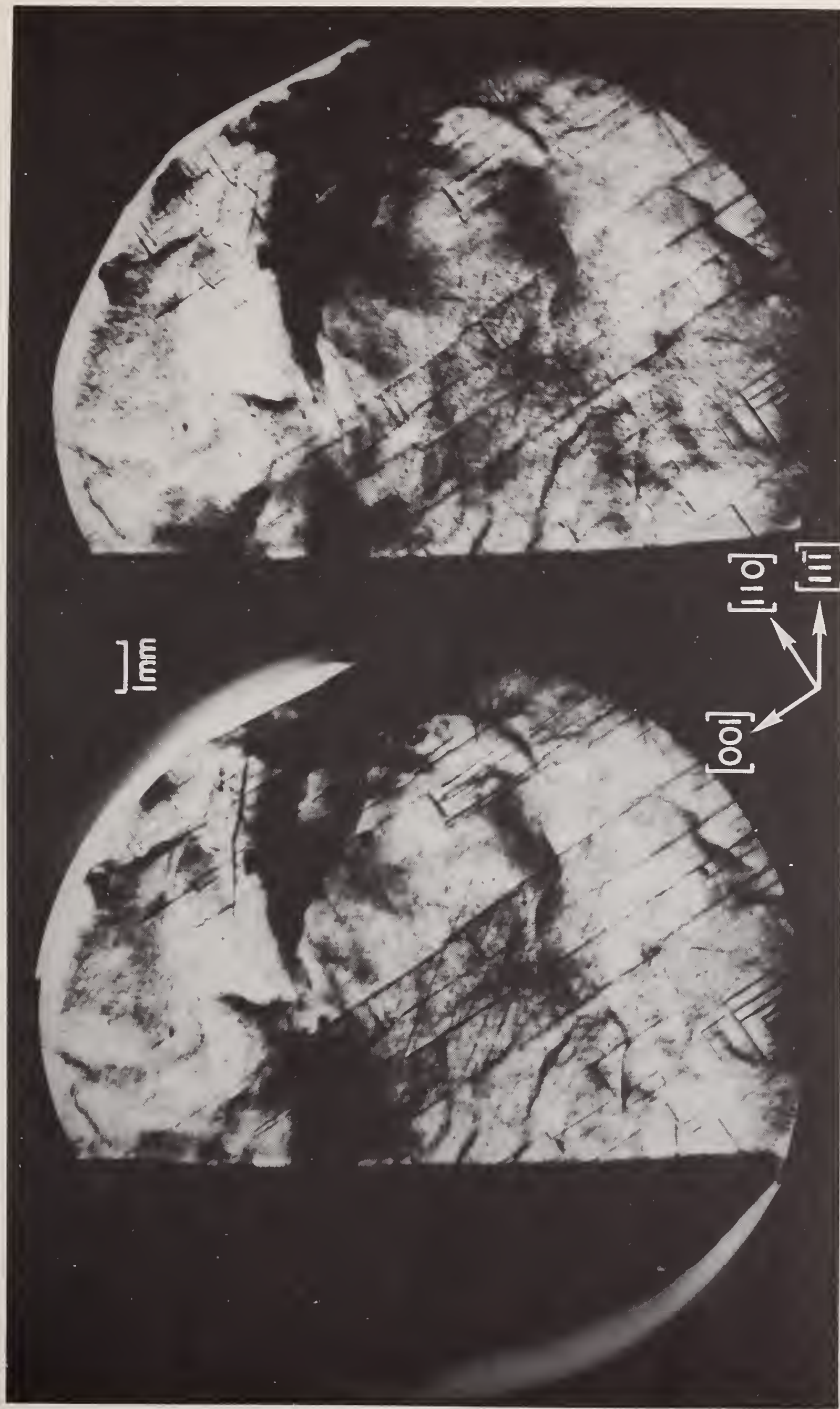





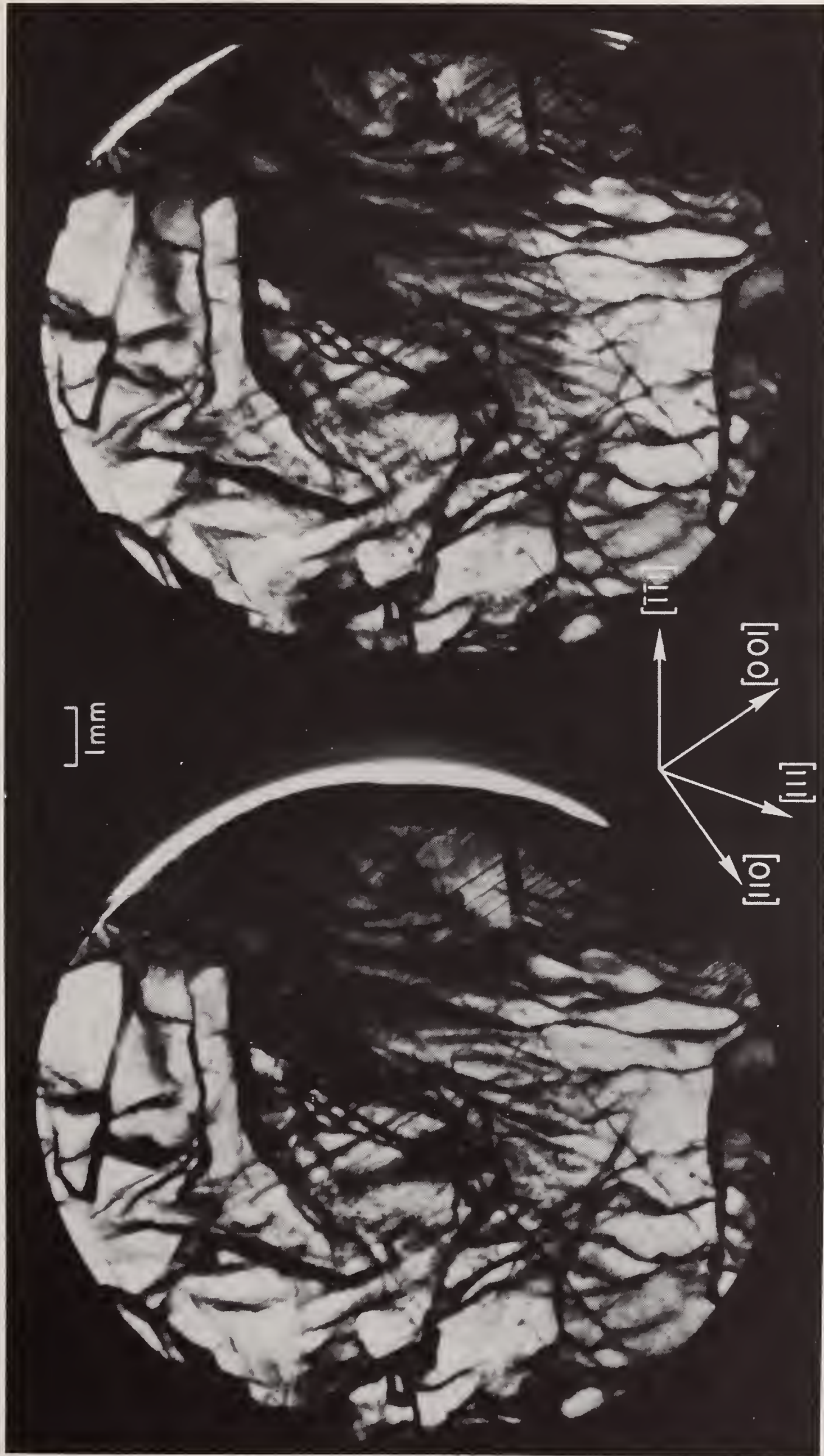

동

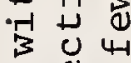

(1)

निम्न

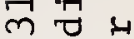

$\checkmark$ o

(2)

○ म

- 100

人

a

- $\rightarrow$ I

다 00

I त)

तु 4

हम क्ष

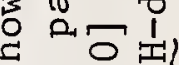

क

صृ

م

수

尔 10 ठ

U

강당

य 8

ᄂ

E

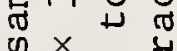

(1) $\Rightarrow$ 出

m $\mathrm{m} \cdot \mathrm{r}$

$\therefore$ त

4

$\circ$ 규

임 थ

م.

का 0

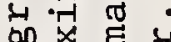

0 ० 0 (1)

م, ㄴ

$\circ$ م

म बि. 0

今

- 0 O 00

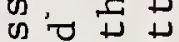

걸 건

है

घ 4

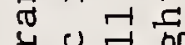

(ठ) मू

( $)$ म

年嵒

न बोन्ण

सु四

选 ه

i

in

- 



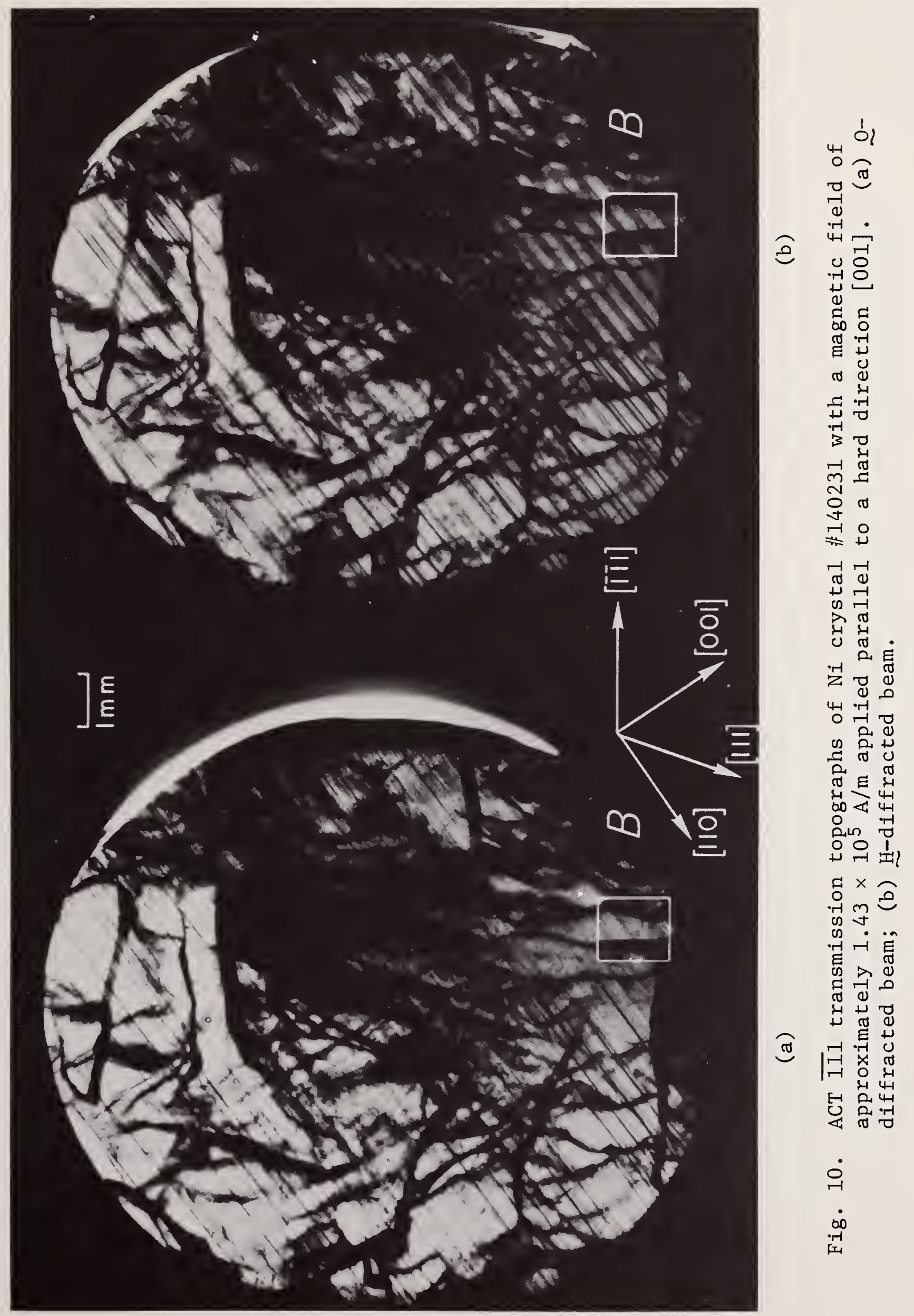





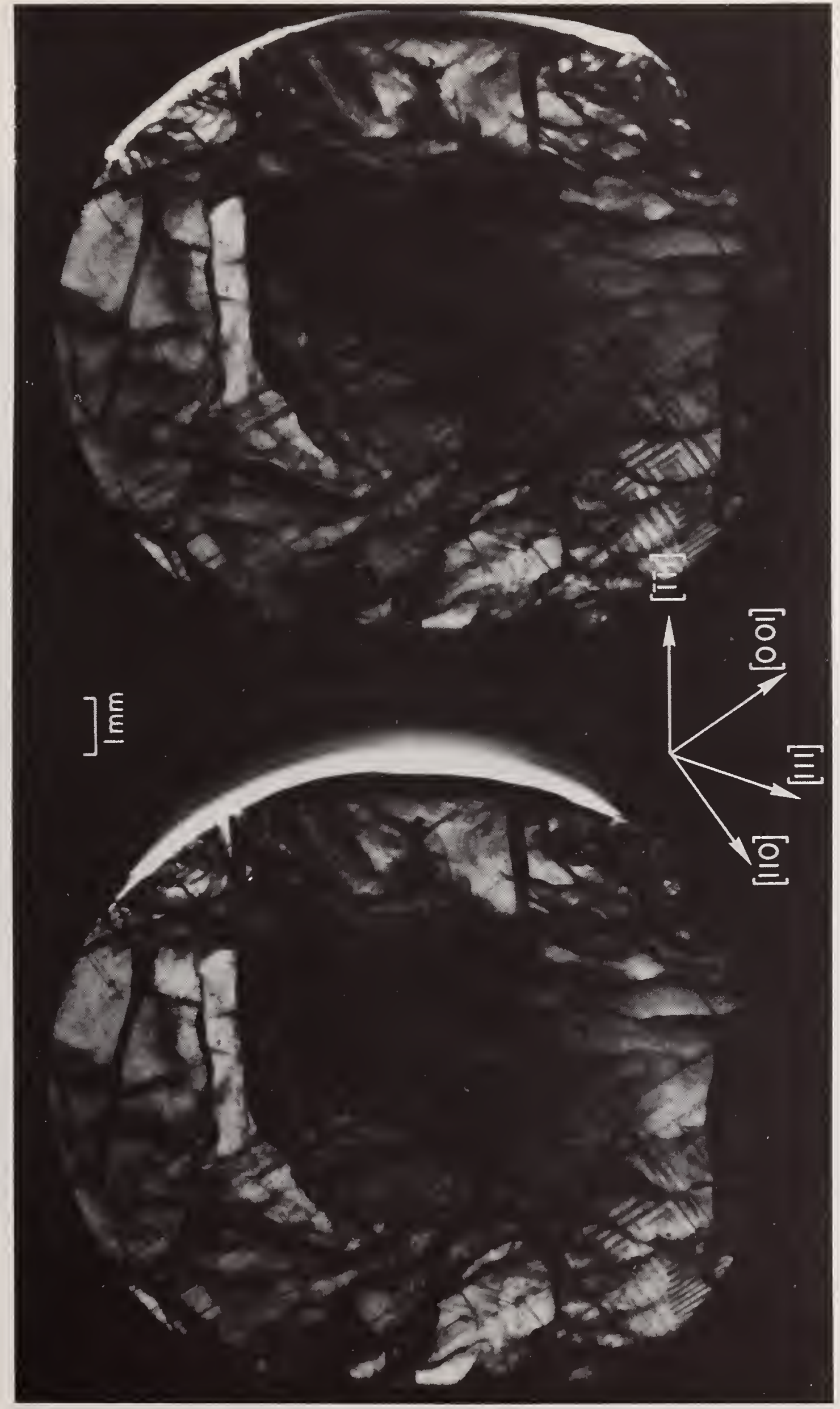

$$
\begin{aligned}
& \text { ن } \\
& \text { (1) } \\
& 3+ \\
& \text { 난 } \\
& \text { 넝 울 } \\
& \text { อ 뭉 } \\
& \text { 언 용 } \\
& \text { 捳 } 0 \\
& \text { ○ స } \\
& \text { - } \\
& \text { 诌 } \\
& \text { 동ㅇㅇㄷㅏ } \\
& \text { 亘红! } \\
& \text { 잉 } \\
& \sum_{-1+1}^{5}+2 \\
& \text { उ } \\
& \text { 다그 } \\
& \text { ก } \\
& \text { ช้ } \\
& \text { \& } \\
& \text { * } \\
& -10 \\
& 7 \text { on } \\
& \text { 雨范 } \\
& \text { o. } \\
& \text { 牙。 } \\
& \text { ० } \\
& \text { 따 } \text { ㅇ } \\
& 0 \text { म } \\
& \text { 匆 } \\
& \text { 요 겅 } \\
& 44 \\
& \text { 吅 } \\
& \text { o } 50 \\
& \text { 응 a0 단 } \\
& + \text { 든 } \\
& \text { 다요 } \\
& \text { 언 } \\
& \text { क } \\
& \text { D) } \\
& \text { - } 5 \\
& \text { ต } \\
& \text { E } \\
& \text { 넌 } \\
& \text { 岇 } \\
& \text { ङ } \\
& \text { - } 0 \text { 星 } \\
& \text { 넝 } \\
& \text { य兄 क } \\
& \stackrel{7}{-1} \\
& \infty_{\substack{\infty \\
-1}}^{\infty} \\
& \text { II }
\end{aligned}
$$





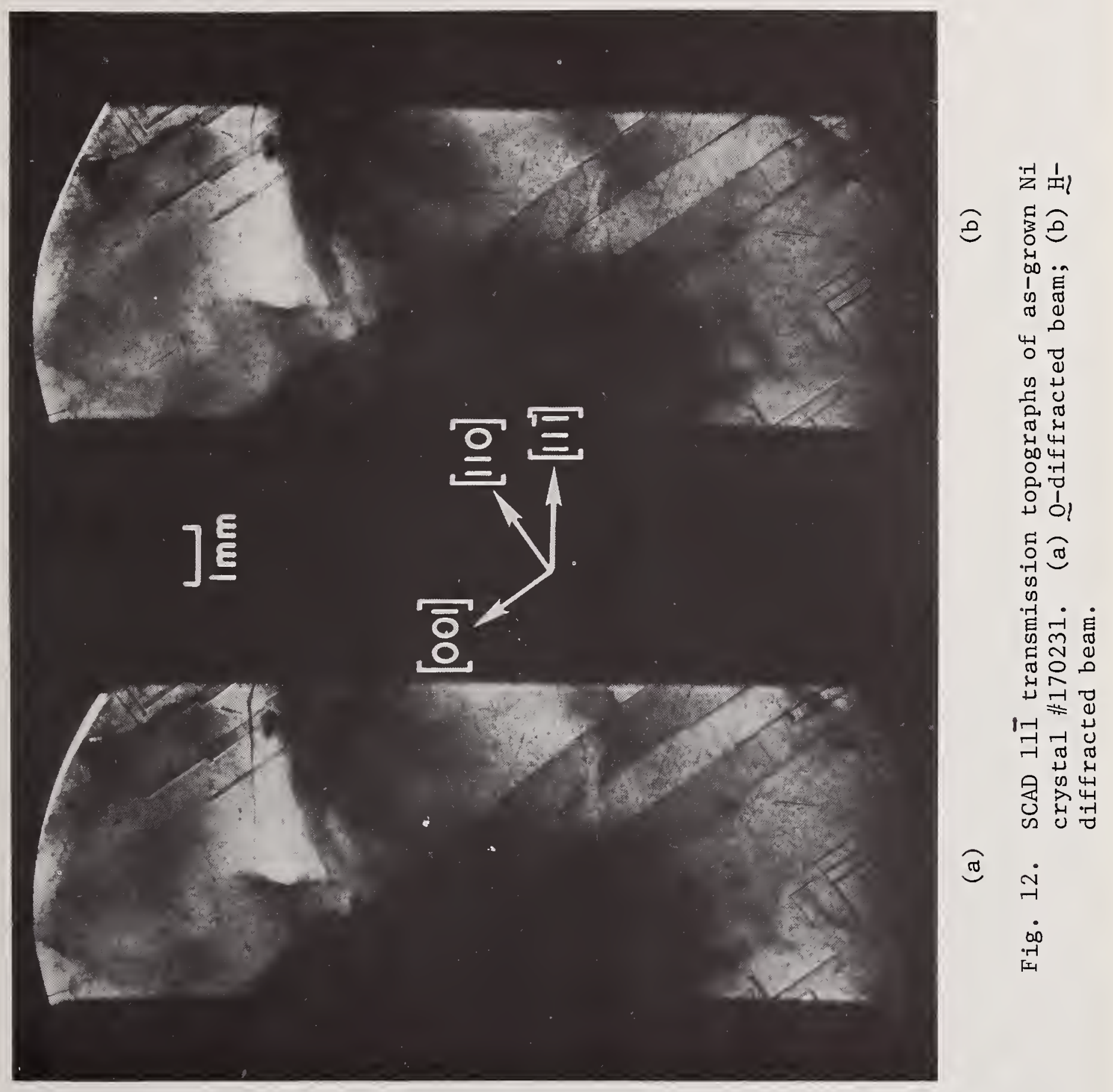



<smiles></smiles>

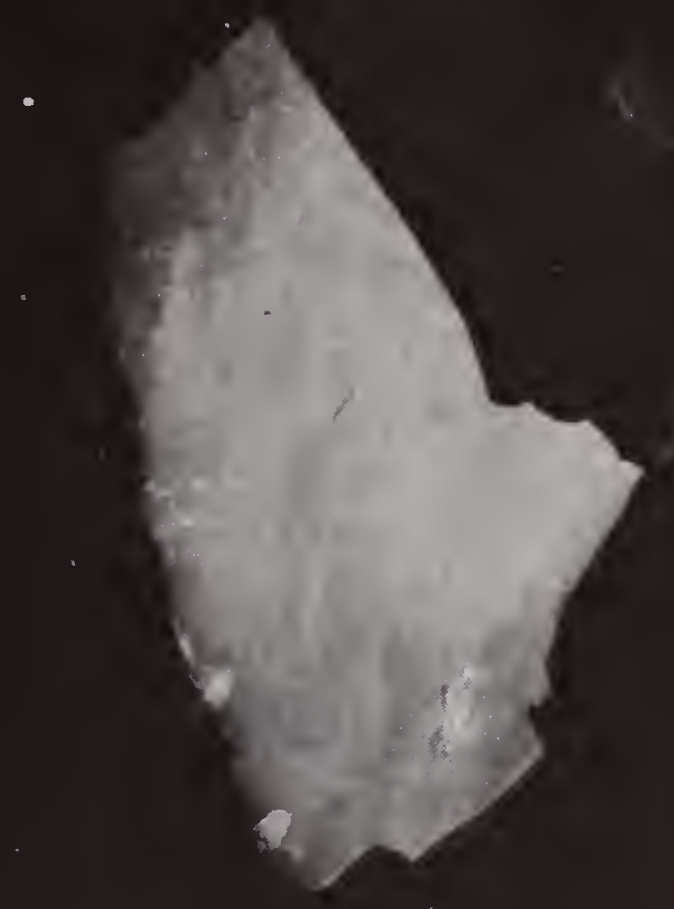

Fig. 13. ACT $2 \overline{2} 0$ surface reflection topograph of $\mathrm{Ni}$ crystal 非194231 grown in the $\langle 001\rangle$ direction. 




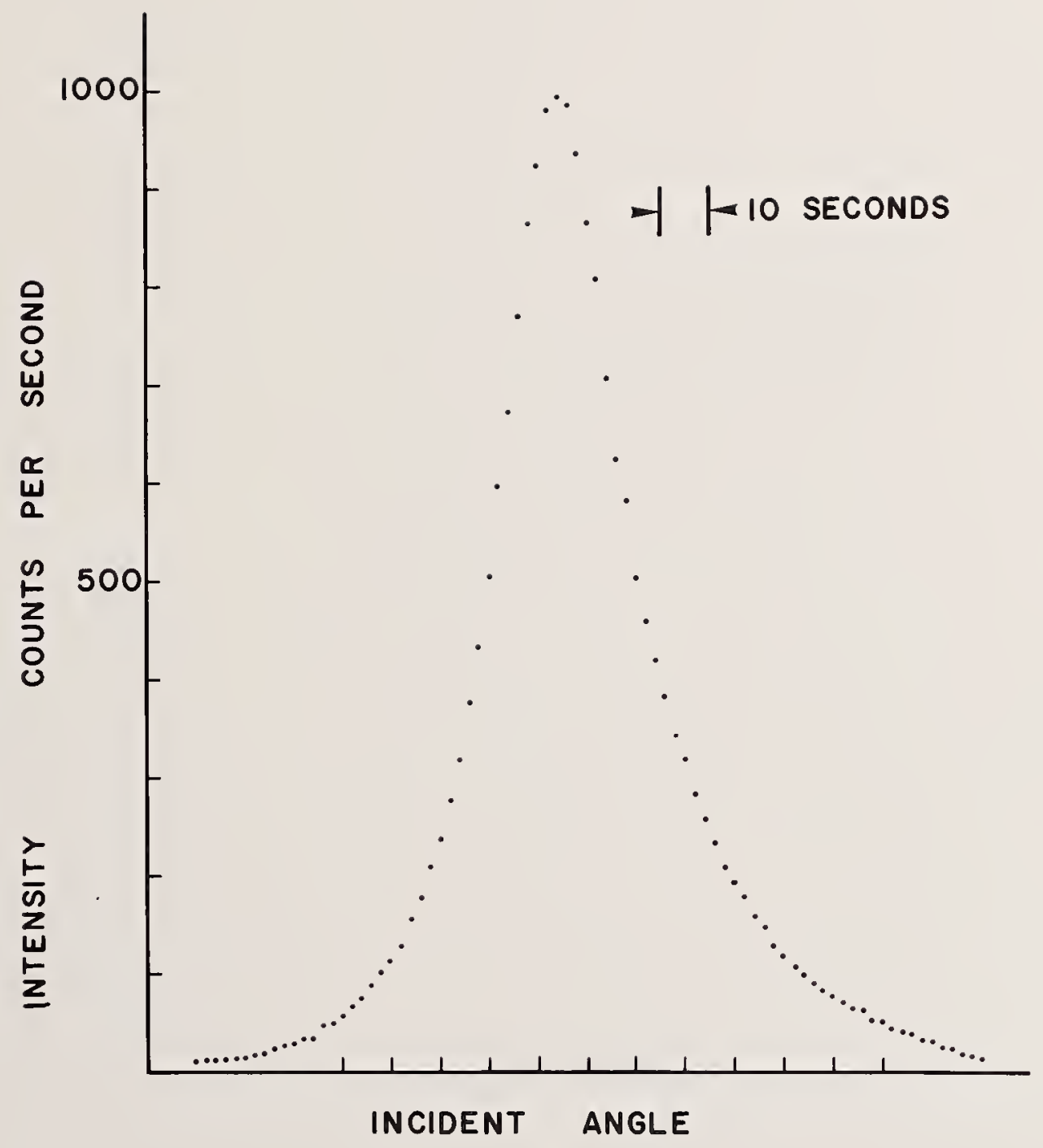

Fig. 15. Rocking curve for the $\overline{2} \overline{2} 0$ diffraction in the symmetric surface reflection geometry. 


\section{$+$}




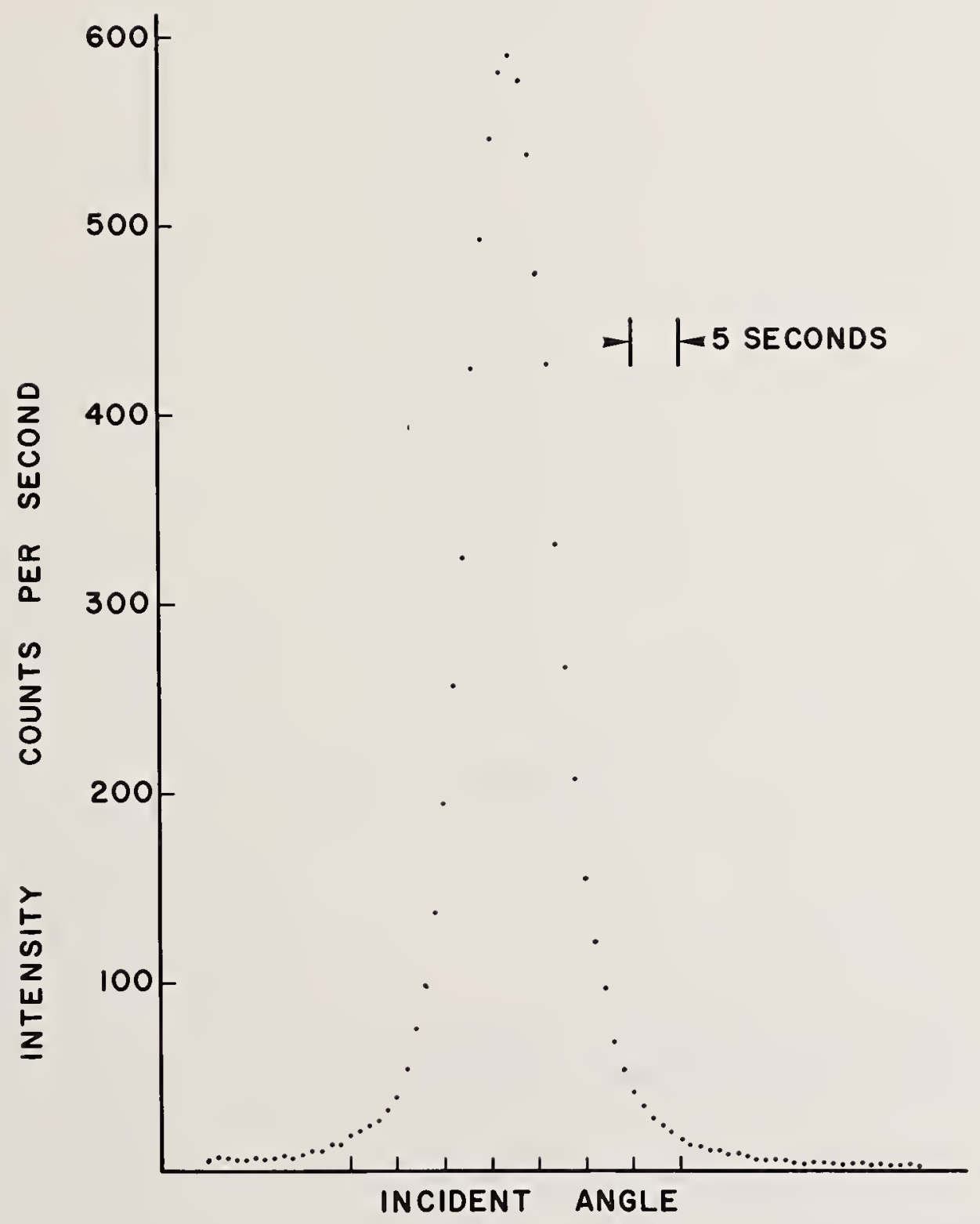

Fig. 16a. Rocking curve for the 111 diffraction in the symmetric transmission geometry. 



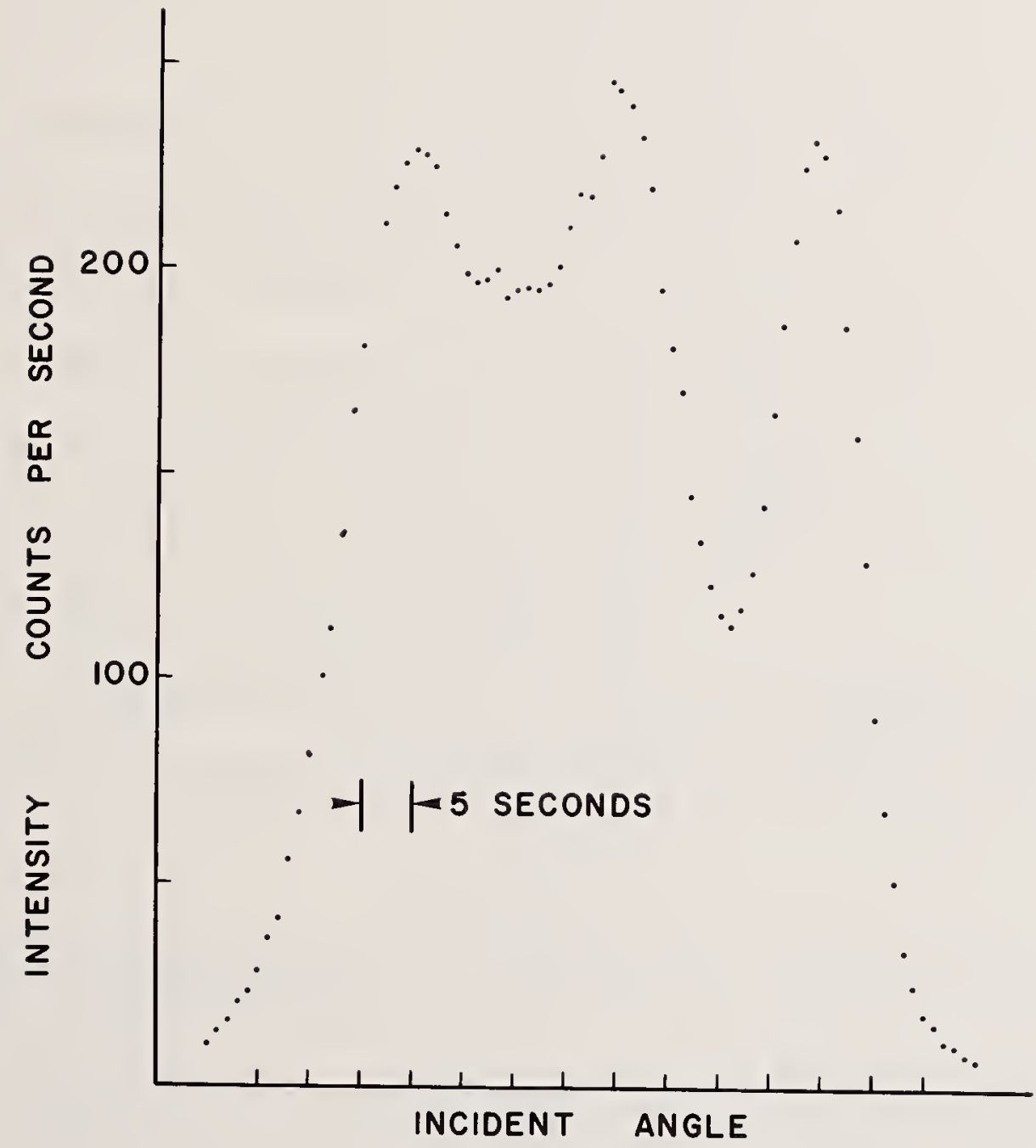

Fig. 16b. Rocking curve for the 111 diffraction in the symmetric transmission geometry for a poor region of the crystal. 

macroscopic arrangements of magnetic domains and the crystal perfection in a more systematic way. If $x$-ray intensities can be made stronger, dynamic behaviors of magnetic domain walls and their distribution can be studied in-situ by $\mathrm{x}$-ray diffraction topography. It is obviously necessary to have nickel single crystals of the present level of perfection or above in such a dynamic investigation. Powerful x-ray sources, such as rotational targets or synchrotron radiations, are available at present. It might not be so far in the future that one could study cyclic magnetization processes by dynamical diffraction topography, if one could systematically produce nickel single crystals better in perfection than those reported here.

In view of these promising important applications, it is extremely desirable to continue the present work in an effort to find whether an optimum choice of the crystal growth conditions improves the resultant crystal perfection, and if so, to evaluate the role of space in producing nickel single crystals of better perfection.

Before concluding this report, we would like to express our appreciation to Roberta M. Eaton and Deborah A. Lavin for their technical assistance.

\section{References}

1. Chikaura, Y., Fukumori, H., and Nagakura, S., Japan J. App1. Phys. 11, 1582-1583 (1972).

2. Alex, V., Tikhonov, L. V., and Brummer, O., Kristall u. Technik 9, 643-645 (1974).

3. Boah, J. K., Metal1, Trans. 4, 1432 (1973).

4. Guterman, M. B., and Trekalo, A. S., Zavodskaya Laboratoriya 34, 823 (1968).

5. Kuriyama, M., Early, J. G., and Burdette, H. E., Proc. of AIAA 12th Aerospace Sciences Meeting, Paper No. 74-204 (1974).

6. Kuriyama, M., Early, J. G., and Burdette, H. E., J. App1. Cryst. $\underline{\text {, }}$ 535-540 (1974).

7. Kurlyama, M., Boettinger, W. J., and Burdette, H. E., J. Appl. Cryst. (1976). 
Task 2

Evaporative Preparation of U1tra-High Purity Materials

\author{
R. C. Paule \\ Inorganic Materials Division \\ W. J. Boettinger and F. S. Biancaniello \\ Metallurgy Division \\ Institute for Materials Research
}

\title{
Summary
}

This Task involves the development of techniques for the ultrapurification of refractory ceramics and metals by high temperature evaporation of volatile contaminants. The near zero-gravity environment of space will be used to allow containerless melting and evaporative purification of relatively large samples, and to provide high vacuum. These ultra-high purity materials may provide future reference materials and materials with unique physical properties. The work reported here is a continuation of NASA contract W-13, 475 非, Task 2 .

Work on evaporative purification is divided into two separate but closely related research areas. One area deals with the mass spectrometric investigation of the detailed mechanisms of the purification process. Problems of measurement associated with purification experiments are also investigated. The second area of research consists of studying the extent of purification that can be obtained in the laboratory using vacuum RF-levitation and heating of small refractory metal samples. In many respects this latter work gives an overall check on the total evaporative purification process. The reporting of work under 
Task 2 has been broken into Parts A and B corresponding to the two general areas of research.

In anticipation of possible evaporative purification experiments with $\mathrm{Al}_{2} \mathrm{O}_{3}$ in space, we have checked the thermodynamically predicted relative abundances of the $\mathrm{Al}_{2} \mathrm{O}_{3}$ vapor species with a mass spectrometer. With the exception of the minor vapor species, $\mathrm{AlO}_{2}$, the predictions were verified.

In order to more accurately predict and control $\mathrm{Al}_{2} \mathrm{O}_{3}$ purification conditions, the effect of non-equilibrium radiative heating on Langmuir (open) vaporization of $\mathrm{Al}_{2} \mathrm{O}_{3}$ has been investigated. This has lead to the development of a radiative heating model to describe the purification experiments both in the laboratory and in space. The model allows us to anticipate moderate temperature measurement problems for $\mathrm{Al}_{2} \mathrm{O}_{3}$ space experiments. An alternate, mass spectrometric, method for the measurement of temperature is suggested. Future work will center on extending the mass spectrometric measurements and the radiative heating model to other chemical systems which show promise for evaporative purification. The goal of the R.F. levitation experiments is to determine the rate of purification of selected metallic systems under conditions which can be conveniently compared to theory. In many evaporative purification experiments reported previously, the rate of purification has been limited by transport of impurities to the evaporation surface rather than by the evaporation process itself. This transport is often impossible to describe quantitatively and leads to experimental data which are difficult to interpret. It is of ten assumed that the stirring induced 
by R.F. fields is sufficient to prevent transport limitations. However, for highly volatile impurities, this assumption has not been previously examined. In this report the purification rate of $\mathrm{Fe}$ from Mo samples has been measured. The Fe impurity was rapidly reduced from $50 \mathrm{ppm}$ to $50 \mathrm{ppb}$ and insight into the degree of mixing present in R.F. levitated melts has been obtained. Because of this insight, future experiments on less volatile impurities can be interpreted on the basis of evaporation processes and not on liquid transport processes. 
Part A: Mass Spectrometric Studies of Vaporization Processes

\section{Introduction}

It is anticipated that future vehicles such as the Space Shuttle will allow processing and manufacturing of unique materials in space. ${ }^{1,2}$ Greater isolation for processes such as evaporative purification of refractory materials will be possible. In vacuum and in near gravityfree space we should be able to conveniently levitate and purify materials such as molten $\mathrm{Al}_{2} \mathrm{O}_{3}$. This technique will eliminate two common sources of contamination, i.e., the ambient gas atmosphere and the container. Such isolation is highly advantageous, but it requires that one know the non-equilibrium radiative and evaporative properties of the material; one can no longer use conventional backscattering processes to produce effective unit emittance and unit evaporation coefficients. We have studied these non-equilibrium properties in order to better understand the control parameters needed for possible future space processing.

The evaporation of solid or molten $\mathrm{Al}_{2} \mathrm{O}_{3}$ under equilibrium conditions has been the subject of numerous investigations. ${ }^{3-9}$ For nonequilibrium conditions there have been fewer studies ${ }^{10-15}$ and the reported $\mathrm{Al}_{2} \mathrm{O}_{3}$ evaporation coefficients have varied from $<0.005$ to 1 . Upon melting the non-equilibrium vaporization of $\mathrm{Al}_{2} \mathrm{O}_{3}$ has been reported to increase discontinuously by a factor of about three. ${ }^{8,9}$ This increase in vaporization was attributed to an increase in the evaporation 
coefficient; the value was believed to discontinuously increase from $1 / 3$ to 1 . In our investigation of evaporative purification of molten $\mathrm{Al}_{2} \mathrm{O}_{3}$ we have looked at this discontinuous increase in vaporization and have studied the interaction of the radiative and the evaporative properties. Our investigation indicates, for the case of radiant heating of an $\mathrm{Al}_{2} \mathrm{O}_{3}$ sample, that the observed jump in vaporization is due primarily to the radiative rather than the evaporative properties of $\mathrm{Al}_{2} \mathrm{O}_{3}$. We find that the evaporation coefficient does not change during the melting of $\mathrm{Al}_{2} \mathrm{O}_{3}$.

Experimenta1

Our $\mathrm{Al}_{2} \mathrm{O}_{3}$ studies were made using a new modulated beam quadrupole mass spectrometric facility. The mass spectrometer and associated furnace are contained in a single, non-partitioned vacuum chamber. The stainless steel chamber is pumped by two liquid-nitrogen-trapped, 4" oil diffusion pumps (see Fig. 1). A tungsten coil resistance furnace is contained in the lower portion of the vacuum system and is surrounded by a water-cooled inner enclosure. The maintenance of cold walls for this inner enclosure has largely eliminated the common problem of furnace outgassing. A large annular opening is included in the bottom of the furnace enclosure so that the furnace can be pumped. Background gases can readily equilibrate between the furnace and the mass spectrometer regions, and ion gauges in the two regions show essentially identical readings. This equilibration minimizes the modulated beam detection of background gases. A $6 \mathrm{~mm}$ diameter opening in the top of the furnace enclosure allows direct effusion of vapors from the hot sample to the mass spectrometer. The effusing sample vapors can be shuttered from the 
mass spectrometer ion source by use of a small movable paddle. The sample vapors, after passing the region of the movable paddle, are chopped by a rotating 10-tooth, 50\% transmission disk. A synchronous motor containing commercial dry $\mathrm{MoS}_{2}$ bearings drives the disk. The dry $\mathrm{MoS}_{2}$ bearings were found to be very suitable for vacuum service.

The neutral molecular beam of vapor products, after being chopped, passes on to the mass spectrometer where the mass-selected AC signal is measured by a synchronized lock-in amplifier. The usual massselected DC signals from the background gases are also simultaneously measured. Background pressures during the experiments were normally about $1.3 \times 10^{-5} \mathrm{~Pa}\left(1 \times 10^{-7}\right.$ torr $)$. The mass spectrometer was usually operated using electron beam energies of 20-22 volts.

To measure the temperature, the sample is viewed by an optical pyrometer through a window in the top vacuum flange. This window looks directly through the ion source of the mass spectrometer and onto the hot sample. Sample brightness temperature measurements were made with the optical pyrometer and simultaneous $\mathrm{Al}_{2} \mathrm{O}_{3}$ vaporization measurements were made with the modulated-beam mass spectrometer.

Two types of solid and liquid sample containment systems were used in the furnace. In the first type, a solid $20 \mathrm{~mm}$ long $\times 3 \mathrm{~mm}$ diameter polycrystalline $\mathrm{Al}_{2} \mathrm{O}_{3}$ rod was positioned such that only the top of the rod was heated and melted, and the liquid $\mathrm{Al}_{2} \mathrm{O}_{3}$ was then contained as a sessile drop. The mass spectrometer could see the fixed area on the top surface of the sessile drop. For the second type of sample containment, a $12 \mathrm{~mm}$ long $\times 6 \mathrm{~mm}$ diameter Re rod with a $1 \times 1 \times 3 \mathrm{~mm}$ long 
slot machined into the top surface was used as the sample holder. This slot, which was tightly and fully packed with $\mathrm{Al}_{2} \mathrm{O}_{3}$ powder also presented a fixed area for vaporization. Both types of containment systems allowed for uninhibited (Langmuir) vaporization. In regard to radiation, both sample containment systems were far from equilibrium or black-body conditions.

\section{Results}

Several interesting radiation and evaporation phenomena occur when the top of an $\mathrm{Al}_{2} \mathrm{O}_{3}$ rod is melted and resolidified. The sample upon melting shows a large drop in its brightness temperature and upon resolidification a large increase in brightness temperature. The corresponding mass spectrometric measurements show a large increase in $\mathrm{Al}_{2} \mathrm{O}_{3}$ vaporization upon melting and an even larger decrease in vaporization upon resolidification (see Fig. 2). This behavior was observed for all vapor species ( $\mathrm{Al}, \mathrm{Al} 10, \mathrm{Al}_{2} \mathrm{O}, \mathrm{Al}_{2} \mathrm{O}_{2}$, and 0 ). The general qualitative features of these phenomena were reproduced upon repeated meltings and resolidifications.

These phenomena have previously been observed, and the factor of three change in vaporization was attributed to a discontinuous change in the evaporation coefficient in going through the melting point. 11,12 It has been claimed that, upon melting, the coefficient changes in value from $1 / 3$ to 1 . There is no a priori reason to question the possibility of such a discontinuous change in the evaporation coefficient. There are, however, a number of a posteriori reasons to question the 
evaporation coefficient explanation for the case of $\mathrm{Al}_{2} \mathrm{O}_{3}$.

The first evidence that the evaporation coefficient explanation is not correct is found in the observation that the vaporization increase upon melting is swaller than the decrease upon resolidification. The evaporation coefficient explanation would require symmetrical processes. Furthermore, the vaporization increase upon melting was found not to be constant for repeated sample meltings, but always increased. During repeated meltings it increased from an initial factor of about three to a factor of about ten.

The above observations, including the associated large changes in sample brightness temperatures, suggest that a rapidly changing sample temperature may be the primary causc of the vaporization "discontinuities." A slotted Re rod was therefore used to contain the $\mathrm{Al}_{2} \mathrm{O}_{3}$ and to act as a temperature moderator for the sample. Under these conditions where the sample temperature could not change rapidly, the vaporization of $\mathrm{Al}_{2} \mathrm{O}_{3}$ did not show a discontinuity (see Fig. 3). Identical results were obtained in repeated experiments. Melting was visually observed during the experiments. Free vaporization conditions were maintained with the $\mathrm{Re}$ cell since the liquid $\mathrm{Al}_{2} \mathrm{O}_{3}$ level was maintained near the top of the shallow slot.

The above measurements show that the evaporation coefficient of $\mathrm{Al}_{2} \mathrm{O}_{3}$ must be constant, and they also suggest that any rapid increase in vaporization is caused by a corresponding rapid increase in surface temperature of the melt due to a discontinuous increase in the emissi- 
vity. There is additional reported evidence to substantiate an increase in the emissivity. ${ }^{12,15,16}$ A greater emissivity of the melt would result in the sessile drop being more efficient in absorbing radiation from the hot furnace coil and this in turn can cause a large increase in the temperature of the melt. A temperature rise is possible since the sample is not in a black-body environment. It should be noted that Langmuir vaporizations, by their very nature, should be done in a relatively open furnace and are therefore not normally made under black-body conditions. The unusual observed sample brightness temperatures shown in Figs. 2 and 3 are due to the lack of black-body conditions. Further discussion of these temperatures, and of the probable value for the emissivity of liquid $\mathrm{Al}_{2} \mathrm{O}_{3}$, will be deferred until a later section.

Mass spectrometric measurements, using 70 volt electrons, were made of the relative pressures of species vaporizing from an $\mathrm{Al}_{2} \mathrm{O}_{3}$ sessile drop held at about $2450 \mathrm{~K}$. All vapor species signals were modulated and all showed a shutter (paddle) effect indicating that they were vaporization products. from the $\mathrm{Al}_{2} \mathrm{O}_{3}$ sample. The observed pressures relative to the $\mathrm{A} 1$ vapor species given in Table I were determined using Mann's cross sections 17 and the usual inverse square root relationship for the multiplier correction. Relative pressures calculated ${ }^{18}$ from the JANAF Tables ${ }^{19}$ are also listed. With the exception of $\mathrm{AlO}_{2}$, we obtained reasonable agreement between the observations and the 1iterature. Regarding $\mathrm{AlO}_{2}$, we saw no evidence for its existence. To minimize the possibility of ion fragmentation, the M.S. electron energy was reduced and a search was made using $10,12,14,16,18,20$, and 22 volt electrons. $\mathrm{A}_{10}$ was not 
observed. The species $\mathrm{Al}_{2} \mathrm{O}_{2}$ with a pressure $1 / 50$ th that reported for $\mathrm{AlO}_{2}$ was easily seen. With our mass spectrometer, it is believed that we could have detected $\mathrm{AlO}_{2}$ at pressures $1 / 500$ th that reported in the Iiterature. Earlier mass spectrometric workers $4,6,7$ have also not seen $\mathrm{AlO}_{2}$, but have seen $\mathrm{Al}_{2} \mathrm{O}_{2}$

\section{$\underline{\text { Table I }}$}

Relative Pressures for Vacuum Vaporization of $\mathrm{Al}_{2} \mathrm{O}_{3}$ at $2450 \mathrm{~K}$

\begin{tabular}{lll} 
& \multicolumn{2}{c}{$\mathrm{P}_{\text {species }} / \mathrm{P}_{\mathrm{AI}}$} \\
\cline { 2 - 3 } Vapor Species & $\underline{\text { Observed }}$ & JANAF \\
$\mathrm{A} 1$ & 1.0 & 1.0 \\
0 & 1.4 & 1.3 \\
$\mathrm{~A} 10$ & 0.17 & 0.36 \\
$\mathrm{Al}_{2}{ }^{0}$ & 0.036 & 0.094 \\
$\mathrm{Al}_{2}$ & -- & 0.012 \\
$\mathrm{Al}_{2}{ }_{2}$ & 0.00023 & 0.00026
\end{tabular}

Thermodynamic values for the vapor species $\mathrm{AlO}_{2}$ have only been estimated by JANAF, and $\mathrm{AlO}_{2}$ has been seen by only one laboratory. This laboratory used a vacuum enclosure with a partition between the furnace and the mass spectrometer regions and it appears that a mass 59 background gas species may have been misidentified as $\mathrm{AlO}_{2}$. If the furnace (mass 59) background pressure were higher than that in the mass spectrometer region, then this background gas would be chopped and detected as a modulated bean. Identification of $\mathrm{Al}_{2} \mathrm{O}_{3}$ vapor species is 
especially hazardous since there is no significant isotopic distribution for either of the elements, Al or 0 .

The absence of the vapor species $\mathrm{AlO}_{2}$ will have a small effect upon the total rate of $\mathrm{Al}_{2} \mathrm{O}_{3}$ vaporization since the reported $\mathrm{AlO}_{2}$ partial pressure was quite small (see Fig. 4).

In the current work, appearance potential measurements were made for the vapor species $A 1, C, \Lambda 10, \mathrm{Al}_{2} \mathrm{O}$, and $\mathrm{Al}_{2} \mathrm{O}_{2} \cdot$ The appearance potential values obtained were $6.0 \pm 1,14.2 \pm 1,10.3 \pm 1,8.1 \pm 1$, and $10.0 \pm 1$, respectively, and are in agreement with the literature. 7,20

Some evidence of evaporative purification was noted during the initial heating of the $\mathrm{Al}_{2} \mathrm{O}_{3}$ samples. Modulated beam signals were initially seen at 40,44 , and 56 amu and are attributed to $\mathrm{CaO}, \mathrm{SiO}_{2}$ and FeO impurities. Rough calculations indicate that the initial respective concentrations were about 200,3 , and $3 \mathrm{ppm}$. The samples were heated at $1900 \mathrm{~K}$ for several days and the impurities were vaporized. prior to melting the $\mathrm{Al}_{2} \mathrm{O}_{3}$. Discussion of Results

Emissive and radiative heat transfer calculations have been made to evaluate the sample and furnace environment. The Langmuir (open) vaporization of the $\mathrm{Al}_{2} \mathrm{O}_{3}$ was not under black-body conditions and as a result the calculations should be thought of as only semi-quantitative in nature. From the radiation viewpoint, the experimental furnace arrangement was complex and the effective emissive characteristics of the sample and furnace were not known with great accuracy. Nevertheless, several large and important thermal effects can clearly be seen to be operating during the melting of $\mathrm{Al}_{2} \mathrm{O}_{3}$. 
To help understand the cause of the thermal effects we have formulated a radiative heating model. For our system let us consider an energy balance per unit time for the top end section of the sample that is to be later melted. Energy to this volume is received only in the form of radiation, $\mathrm{R}$, from the hot tungsten furnace coil. Note that $\mathrm{R}$ is that part of the total furnace radiation that hits the sample volume under consideration. The energy losses from this volume can be grouped into three categories: (1) reflected radiation, $p R$, where $\rho$ is the total reflectance of the sample, (2) emitted radiation, $\epsilon \mathrm{r}_{\mathrm{bb}}$, where $\epsilon$ is the total emittance and $r_{b b}$ is the associated black-body radiation of the sample, and (3) energy lost by thermal conduction down the $\mathrm{Al}_{2} \mathrm{O}_{3}$ rod. The energy loss by thermal conduction is a function of the sample geometry and the sample temperature. For a relatively constant geometry and temperature, the thermal conduction losses can be expressed as a constant, $k$, times $r_{b b}$. By combining the three sources of energy loss we obtain the following energy balance equation:

$$
\mathrm{R}=\rho \mathrm{R}+\epsilon \mathrm{r}_{\mathrm{bb}}+\mathrm{kr}_{\mathrm{bb}} \text {. }
$$

Since the sample is normally either polycrystalline $\mathrm{Al}_{2} \mathrm{O}_{3}$ or a melt, it is essentially opaque and we can replace the total reflectivity by the term $1-\epsilon$.

$$
R=(1-\epsilon) R+\epsilon r_{b b}+k r_{b b}
$$


Rearranging the terms we obtain:

$$
R=\left(\frac{\epsilon+k}{\epsilon}\right) r_{b b} .
$$

The thermal conductivity for our solid $\mathrm{Al}_{2} \mathrm{O}_{3}$ rod results in a $\underline{k}$ value of about 0.11 .21 The total emissivity of solid $\mathrm{Al}_{2} \mathrm{O}_{3}$ just below the melting point is about $0.35^{22}$ while the value for molten $\mathrm{Al}_{2} \mathrm{O}_{3}$ is about $0.90 .15,16$ Equation (3) for solid $\mathrm{Al}_{2} \mathrm{O}_{3}$ just below the melting point becomes

$$
\mathrm{R}=\left(\frac{.35+.11}{.35}\right) \mathrm{r}_{\mathrm{bb}}=1.31 \mathrm{r}_{\mathrm{bb}} \text {. }
$$

When an infinitesimally small increment of radiation from the furnace is added, the $\mathrm{Al}_{2} \mathrm{O}_{3}$ melts and we obtain:

$$
\mathrm{R}+\mathrm{dR}=\left(\frac{.90+.11}{.90}\right) \mathrm{r}_{\mathrm{bb}}^{\prime}=1.12 \mathrm{r}_{\mathrm{bb}}^{\prime}
$$

where $r^{\prime}{ }_{b b}$ refers to the melt. (The heat of fusion required for melting the $\mathrm{Al}_{2} \mathrm{O}_{3}$ is a transient effect and is not considered in this development of the radiative heating model.) Equating the furnace input radiation from eqs. (4) and (5), and rearranging we obtain:

$$
\frac{r_{b b}^{\prime}}{r_{b b}}=\frac{1.31}{1.12}=1.17
$$

By using the Stefan-Boltzman law, and the melting point of $\mathrm{Al}_{2} \mathrm{O}_{3}{ }^{23}$ as a point of temperature calibration, we obtain:

$$
\frac{\mathrm{r}_{\mathrm{bb}}^{\prime}}{\mathrm{r}_{\mathrm{bb}}}=1.17=\left[\frac{\mathrm{T}^{\prime}}{2327 \mathrm{~K}}\right]^{4} \text {. }
$$


Solving:

$$
\mathrm{T}^{\prime}(\mathrm{melt})=2420 \mathrm{~K}
$$

and

$$
\Delta T=T^{\prime}-2327 \mathrm{~K}=93 \mathrm{~K} .
$$

Thus, the melt can be expected to spontaneously increase in temperature to about $90 \mathrm{~K}$ above the melting point. This increase is due to the combined effect of the thermal conductivity losses and the significantly higher total emissivity of the melt.

A $90 \mathrm{~K}$ temperature rise corresponds to a factor of three increase in the $\mathrm{Al}_{2} \mathrm{O}_{3}$ vapor pressure (see $\mathrm{Fig} .4$ ). This closely corresponds to the observed vapor pressure increase upon melting. A $90 \mathrm{~K}$ temperature rise should result in all $\mathrm{Al}_{2} \mathrm{O}_{3}$ vapor species increasing by nearly the same amount. Our observations agree with this conclusion.

At this point in the model experiment the molten $\mathrm{Al}_{2} \mathrm{O}_{3}$ is considerably above the melting point and the furnace power can be reduced somewhat without refreezing the $\mathrm{Al}_{2} \mathrm{O}_{3}$. One should be able to reduce the furnace power and the melt temperature until the freezing point temperature (and vapor pressure) are obtained. At that point the $\mathrm{Al}_{2} \mathrm{O}_{3}$ should refreeze and the temperature should drop considerably below the freezing point since the refrozen $\mathrm{Al}_{2} \mathrm{O}_{3}$ should have a lower radiation efficiency (lower total emissivity). In practice the general features of this description are followed (see Fig. 2). The commencement of freezing, however, appears to be somewhat early relative to the model in that it starts at too high a vapor pressure (temperature). This point of 
transition may involve a more sensitive energy balance and a more detailed picture is probably required. Furthermore, the actual refreezing process is not truly reversible in that a clear, transparent sapphire bead forms rather than the original polycrystalline $\mathrm{Al}_{2} \mathrm{O}_{3}$. The development of a more detailed energy balance to describe this more involved situation was not pursued since it requires significantly more emissivity information, much of which is not currently available.

Let us now examine some of the effects that the thermal conductivity constant, $k$, has upon the experiments. If there were no thermal conductivity $\left(\mathrm{k}=0\right.$ ) then Eq. (3) tells us that $\mathrm{R}=\mathrm{r}_{\mathrm{bb}}=\mathrm{r}_{\mathrm{bb}}{ }_{\mathrm{b}}$ and there would be no temperature jump upon melting. Conversely, if $\mathrm{k}$ should become larger then the temperature jump should become larger. During repeated remeltings (and vaporizations) the $\mathrm{Al}_{2} \mathrm{O}_{3}$ rods used in our experiments became shorter and as a result the $\mathrm{k}$ values became larger. In agreement with the model, the observed temperature (and vaporization) jumps did become larger with repeated remeltings. It should be noted, however, that the remeltings were of sapphire beads formed from the previous melts. While the partial transparency of sapphire complicates the analysis, it is believed that the general processes, reasoning and conclusions would be the same.

The current model, in addition to explaining the sample temperature jump, provides a qualitative explanation of the observed brightness temperatures. The observed brightness of the sample is due to two radiation sources, i.e., the emitted and the reflected radiation. The reflected radiation is from the tungsten furnace coil which is about $500 \mathrm{~K}$ hotter 
than the sample. The furnace coil must be much hotter than the sample since the furnace is quite open and there is relatively low efficiency in radiative heat transfer to the sample. One consequence of this higher temperature is that the spectra from the furnace is considerably richer in 6500 A radiation than that emitted from the sample. Therefore for a condition of equal total radiation, $\mathrm{e} \cdot \mathrm{g} \cdot, \mathrm{R}=\mathrm{r}_{\mathrm{bb}}$, an optical pyrometer operating at $6500 \AA$ will see considerably more radiation from the furnace than from the emitting sample. Equations (4) and (5) show that the condition of equal total radiation $\left(R=r_{b b}\right)$ will be approximately true for our experiments.

Let us now examine what happens when polycrystalline $\mathrm{Al}_{2} \mathrm{O}_{3}$ melts. The radiation measured by the pyrometer should be proportional to

$$
\rho R+\epsilon r_{b b}=(1-\epsilon) R+\epsilon r_{b b}
$$

where we now consider the $\rho, 1-\epsilon, R$, and $r_{b b}$ values to be evaluated at $6500 \AA$. The spectral emissivity values for polycrystalline $\mathrm{Al}_{2} \mathrm{O}_{3}$ and the melt are about $.45^{20}$ and $.90,13,14$ respectively. The 6500 A radiation to the pyrometer, except for the fixed window-prism correction and the fixed geometry factor, is therefore

$$
.55 \mathrm{R}+.45 \mathrm{r}_{\mathrm{bb}} \quad \text { (polycrystalline } \mathrm{Al}_{2} \mathrm{O}_{3} \text { ) }
$$

and

$$
.10 \mathrm{R}+.90 \mathrm{r}_{\mathrm{bb}}^{\prime} \quad\left(\mathrm{Al}_{2} \mathrm{O}_{3} \mathrm{melt}\right) .
$$

Since the $\mathrm{R}$ of the furnace at $6500 \stackrel{\mathrm{A}}{\mathrm{i}}$ is considerably larger than either $r_{b b}\left(\right.$ or $r_{b b}^{\prime}$ ) of the sample, relationships 6 and 7 show that the 
measured optical pyrometer brightness temperature for the melt will be less than that for the polycrystalline $\mathrm{Al}_{2} \mathrm{O}_{3}$. This is observed in all of our temperature measurements (see Figs. 2 and 3). The exact calculation of the predicted brightness temperature, however, is subject to moderate uncertainties since we can only estimate $R$ at $6500 \AA$ A. Equations (4) and (5) are of no direct aid to us in this regard since the $\mathrm{R}$ in these equations refer to the integrated (total) radiation. The direct observation of $\mathrm{R}$ at 6500 A was not possible in our experimental arrangement. Any reasonable estimate, however, for $\mathrm{R}$ at $6500 \AA$ will lead to a lower brightness temperature for the melt than for the polycrystalline $\mathrm{Al}_{2} \mathrm{O}_{3}$

Conclusions (Part A)

It is anticipated that the discontinuous nature of the emittance of $\mathrm{Al}_{2} \mathrm{O}_{3}$ will have several consequences in any space manufacturing venture using this material. Three of the more obvious consequences are:

(1) For an $\mathrm{Al}_{2} \mathrm{O}_{3}$ sample levitated in space, thermal conduction will be absent and there will not be a temperature jump. The observed spectral radiation from the sample at $6500 \stackrel{\mathrm{A}}{\text {, however, }}$ will still depend on both the spectral emittance of the sample and on the spectra of the input (heating) radiation. This will cause difficulties in accurately measuring the temperature with an optical pyrometer.

(2) Mass spectrometric measurement of the $\mathrm{Al}_{2} \mathrm{O}_{3}$ vapor pressure should be a reliable alternate method for continuously monitoring the temperature of the sample since these measurements 
are not affected by the furnace or sample radiation. Since the evaporation coefficient of $\mathrm{Al}_{2} \mathrm{O}_{3}$ is constant, the M.S. vapor pressure measurements can be calibrated at the melting point.

(3) For non-radiative heating of $\mathrm{Al}_{2} \mathrm{O}_{3}$ there will not be a temperature jump. There will, however, still be emissivity effects. For the non-radiative heating case we have the energy balance relationship, energy in = energy out $=\varepsilon \mathrm{r}_{\mathrm{bb}}$, and we still need to accurately know the spectral emissivity to measure the temperature with an optical pyrometer. Again, M.S. vapor pressure measurements should be a useful alternate method for continuously monitoring the temperature of the sample.

Additional conclusions from this work are that the thermodynamic literature is reasonably reliable for predicting the relative abundances of the $\mathrm{Al}_{2} \mathrm{O}_{3}$ vapor species. The literature for the vapor species $\mathrm{AlO}_{2}$, however, is a minor exception to this conclusion.

Finally, our observations have shown that $\mathrm{CaO}, \mathrm{SiO}_{2}$, and $\mathrm{FeO}$ impurities can be vaporized from $\mathrm{Al}_{2} \mathrm{O}_{3}$. 


\section{Introduction}

The purification of metals and alloys by evaporation of volatile impurities is a well-known technique of materials processing and is employed extensively in industry and in the laboratory. The basic principles of evaporative purification and distillation have been known since the nineteenth century. ${ }^{24,25}$ More recently these techniques have been applied to the refining of refractory metals such as $\mathrm{Nb}, \mathrm{Mo}, \mathrm{Ta}$, and $\mathrm{W}$ by various methods of vacuum melting. These refinings are frequently performed in configurations which are convenient but not simple in terms of basic purification processes. Often, transport of the impurities to the evaporating surface (or transport of the vaporized impurity away from the evaporating surface in the case of refining in a poor vacuum or an atmosphere) is the limiting process in the purification rather than the evaporation process itself. Much data has been accumulated for electronbeam zone-refined materials. These data have the additional complications that impurities are removed by evaporation from a non-isothermal sample as well as by zone refining. Even for induction stirred melts in vacuum it has been reported ${ }^{26}$ that there may be insufficient mixing to prevent depletion of the impurity in the liquid near the evaporating surface and this will prevent optimum purification rates. Because of these experimental complexities, rates of purification reported in the literature are difficult to interpret in terms of basic evaporation processes. Yet the success of any evaporative purification scheme, regardless of the details, 
is based on these evaporation processes. Only two extremes of impurity transport in the liquid lead without difficulty to a comparison of evaporation theory to experiment--the case of no impurity transport limitation (completely stirred) and the case of impurity transport by diffusion only. The general approach of this research has been to measure the rate of loss of selected metallic impurities from refractory metals processed by R.F. levitation melting in vacuum. Advantages of levitation melting for this purpose include good stirring, rapid heating to and cooling from temperature, a nearly spherical evaporation surface and the absence of contamination from containers. In addition, this technique is quite similar to proposed methods of positioning and heating refractory materials in a low gravity environment. Purification rates determined with this method should avoid some of the complexities inherent in other evaporative purification configurations and provide an overall check on the total evaporation process.

The primary effort of last year's work ${ }^{2}$ was to develop specific laboratory procedures for the use of R.F. levitation melting for the measurement of purification rates for Mo. In this report, experiments are described in which Mo samples with about 50 ppm Fe were levitation melted in vacuum for various lengths of time and initial and final chemical analysis were used to measure the rate of purification. The impurity $\mathrm{Fe}$ was chosen because it is the most abundant impurity in commercial purity Mo and because its extreme volatility with respect 
to Mo should give insight into whether evaporation or transport of impurity in the liquid is the rate limiting process for the purification of R.F. levitated melts. The determination of this fact is essential before quantitative comparisons of theory and experiment can be performed.

\section{Purification Rate}

In a previous report ${ }^{1}$, calculations for the purification of materials where complex interactions between species are present has been performed. This computer program can be used (in a trivial fashion) to predict the rate of evaporation into vacuum of a well-stirred isothermal ideal binary solution of two metals.* However, in this simple case the rate can be obtained analytically. Analytical solutions are advantageous because they immediately show the effect of parameters on the solution and in this case provide a convenient means of planning and interpreting simple purifications experiments.

Let $n_{A}(t)$ and $n_{B}(t)$ be the number of moles of $A$ and $B$ present in the liquid at time $t$. Assuming Knudsen evaporation conditions apply the relevant equations describing the purification process are:

$$
\left\{\begin{array}{c}
\mathrm{p}_{A} \frac{\mathrm{n}_{A}}{n_{A}+n_{B}}=-k_{A} \frac{d n_{A}}{d t} \\
p_{B} \frac{n_{B}}{n_{A}+n_{B}}=-k_{B} \frac{d n_{B}}{d t}
\end{array}\right.
$$

\footnotetext{
"It should be noted that for Mo-Fe evaporation, the computer solution converged to the analytical solution when the time intervals were short enough. This provided an overall check of the iterative techniques used in the computer program.
} 
where $k_{i}=\frac{\left(2 \pi R \mathrm{Rm}_{i}\right)^{1 / 2}}{A}$ and

$$
\begin{aligned}
& \mathrm{p}_{\mathrm{i}}=\text { vapor pressure of pure } \mathrm{i} \text { at temperature } \mathrm{T} \\
& \mathrm{m}_{\mathrm{i}}=\text { molecular weight of component } \mathrm{i} \\
& \mathrm{A}=\text { area of sample available for evaporation } \\
& \mathrm{R}=\text { universal gas constant } \\
& \mathrm{T}=\text { absolute temperature. }
\end{aligned}
$$

The solution* when A is a constant is given by

$$
\left\{\begin{array}{c}
\frac{\mathrm{n}_{A}}{\mathrm{~N}_{A}}=\left(\frac{\mathrm{n}_{B}}{\mathrm{~N}_{B}}\right)^{\alpha} \\
t=\frac{\mathrm{k}_{\mathrm{B}} \mathrm{N}_{A}}{\mathrm{p}_{\mathrm{B}}^{\circ}}\left\{\frac{\mathrm{N}_{B}}{\mathrm{~N}_{A}}\left(1-\frac{\mathrm{n}_{B}}{\mathrm{~N}_{B}}\right)-\frac{1}{\alpha}\left[\left(\frac{\mathrm{n}_{B}}{\mathrm{~N}_{B}}\right)^{\alpha}-1\right]\right\}
\end{array}\right.
$$

where $\mathrm{N}_{\mathrm{A}}$ and $\mathrm{N}_{\mathrm{B}}$ are the initial number of moles of $\mathrm{A}$ and $\mathrm{B}$ respectively and $\alpha=\left(\mathrm{p}_{A} / \mathrm{p}_{B}\right)\left(\mathrm{m}_{B} / \mathrm{m}_{A}\right)^{1 / 2}$. For a given number of moles of $B$ remaining, the

*Equation (10) is valid even if $\mathrm{A}$ is not constant and even if there are other components in the solution. When $\alpha$ and $\mathrm{N}_{\mathrm{B}} / \mathrm{N}_{\mathrm{A}} \ll 1$, eqs. (10) and (11) are approximated by $\mathrm{n}_{A}=\mathrm{N}_{A}$ and $\mathrm{n}_{\mathrm{B}}=\mathrm{N}_{\mathrm{B}} \exp \left\{-\mathrm{p}_{\mathrm{B}} \mathrm{t} / \mathrm{k}_{\mathrm{B}} \mathrm{N}_{\mathrm{A}}\right\}$. 
number of moles of A remaining is given by Eq. (10) and the time, $t$, required to achieve these levels is given by Eq. (11). From this the concentration can then be simply calculated for any time. As has been known since the nineteenth century, component A can be purified of component B when $\alpha$ is less than one. It must be re-emphasized that this type of solution even extended to multicomponent systems is only valid when there is no chemical reaction of species. For the case where reactions are present, the calculation developed for complex evaporation must be used.

\section{Experimental Procedures}

It was the purpose of previous work ${ }^{2}$ to develop specific procedures suitable for the measurement of purification rates of refractory metals using R.F. levitation melting in vacuum and most of the details are presented in that report. A schematic of the equipment used is shown in Fig. 5. A product of last year's work was the development of a levitation coil which produces high gradients of the magnetic field in the vertical direction. Use of this type of coil enables a modest degree of temperature control of levitated samples.

At the start of the present work, modifications were made to the R.F. generator which increased the maximum power and permitted smoother power control. The increase in power permits experiments to be run for longer periods of time. At constant power setting, the temperature of a levitated sample decreases as the sample gets smaller. Because previously samples were levitated and melted at the maximum power setting, there was only a finite time before the sample would freeze due to this 
temperature decrease. With the added power, we have doubled the length of time that Mo-based alloys can be processed in the liquid state in earth gravity.

A second important improvement has been the use of an automatic optical pyrometer and a vacuum window which prevents the accumulation of metallic vapor. Hence, the temperature of levitated samples is monitored continuously during melting, purification and freezing. The vacuum window consists simply of a transparent teflon tape which moves across a glass vacuum window. The window is $15 \mathrm{~mm}$ in diameter and the tape moves at $15 \mathrm{~mm} / \mathrm{sec}$. The output of the pyrometer along with that of an ionization gauge in the levitation chamber is recorded on a dual channel recorder. The effects of sample emissivity and absorption of the window are calibrated for each experiment using the melting point of Mo as a reference.

A third improvement which is attributed to the coil design and the improved power control of the R.F. generator is the ability to melt and refreeze samples while levitated in vacuum. Much work performed in the past by others required the introduction of a gas into the system to solidify the sample. Currently $4 \mathrm{~g}$ Mo samples can be stably levitated between the temperatures of $2600 \mathrm{~K}$ and $3100 \mathrm{~K}$ (M.P. Mo $\sim 2896 \mathrm{~K}$ ). These accomplishments are very sensitive to sample size. Although the increase in power permits the levitation and melting of Mo samples down to $3 \mathrm{~g}$, the optimum conditions are achieved with samples initially $4 \mathrm{~g}$. Larger samples tend to drip or pour while molten. This limitation will not be present in low gravity environments and much larger quantities of material can be processed. It should be noted here that it was not the purpose of 
this work to engineer the most versatile levitation system for use on earth but rather to employ readily available technology to perform evaporative purification experiments.

A11 purification experiments reported here were performed on etched $4 \mathrm{~g}$ samples taken from a single rod $(8.4 \mathrm{~mm}$ diameter) of commerical purity Mo. Three samples were taken from the rod at different positions along the length and were chemically analyzed to determine the variation of the initial composition of the samples.

Samples for purification were placed in the levitation coil and rested directly on the lower turns of the R.F. coil, and the system was evacuated to less than $7 \times 10^{-5} \mathrm{~Pa}\left(\sim 5 \times 10^{-7}\right.$ torr $)$. The coil was energized rapidly causing the sample to levitate. After melting, the temperature was adjusted nanually to about $3000 \mathrm{~K}$ and held for various lengths of time. Power was reduced causing the sample to freeze and cool to about $2600 \mathrm{~K}$. Then the power was cut off and the sample fell into a copper dish. A typical temperature-time, vacuum-time record (test 3-24) is shown in Fig. 6. After cooling to room temperature, samples were removed from the vacuum and prepared for chemical analysis.

In order to avoid the effects of micro and/or macrosegregation of impurities caused by solidification, chemical analysis was performed on one entire half of the purified samples. The solidified samples were cut in half through the geometrical symmetry axis which these samples generally possess. This axis was vertical in the levitated sample at the instant the sample surface solidified. The sawing was performed using a 
$\mathrm{Al}_{2} \mathrm{O}_{3}$ water-cooled wheel to minimize $\mathrm{Fe}$ contamination. The chemical analyses reported here were performed by the Analytical Chemistry Division of the National Bureau of Standards. Unpurified samples and four of the purified samples were analyzed for $\mathrm{Fe}$ using Extraction-Spectrophotometric analysis. The details of the technique are presented in Appendix I. The sensitivity of this technique is 2 ppm by mass (weight). Because all of the purified samples had $\mathrm{Fe}$ levels below $2 \mathrm{ppm}$, the other halves of these samples as well as an additional nine purified samples were analyzed using Isotope Dilution Mass Spectroscopy*. The details of this technique are given in Appendix II. The sensitivity of this technique (twice the blank Fe concentration) is $50 \mathrm{ppb}$ by weight. This is currently the most accurate technique available for analysis at these levels and it is especially suitable for analysis of bulk samples.

Equipment failures and time required to perform chemical analysis greatly reduced the number of samples processed below the desired number. Three months of experimentation were lost due to failures of the R.F. generator and failure of the optical pyrometer. Additionally, one month was required for the careful chemical analysis reported here. Experimental Results

Table II shows the Fe concentration determined in duplicate for three samples from the starting material. The average iron value is 48.4 ppm by weight. Table II also summarizes the experiments performed and

\footnotetext{
*Four samples were analyzed using both techniques and the results are consistent. (see Appendix I and II.)
} 
TABLF. II - SLYLARY OF EVAPORATIVE FURIFICATION EXFERIMENTS ON MLYBDENLY.

INITIAL MAIER IAL

\begin{tabular}{|c|c|}
\hline $\begin{array}{c}\text { Sample } \\
\text { Number }\end{array}$ & $\begin{array}{c}\text { Fe } \\
\text { Content }\end{array}$ \\
\cline { 2 - 2 } & $\begin{array}{c}\text { npm } \\
\text { (by mass) }\end{array}$ \\
\hline A & 54.0 \\
& 54.2 \\
B & 46.9 \\
& 47.5 \\
C & 43.8 \\
\hline
\end{tabular}

PUR IFIED MATER IAL

\begin{tabular}{|c|c|c|c|c|c|c|c|c|c|c|}
\hline \multirow[t]{2}{*}{$\begin{array}{l}\text { Sample } \\
\text { Number }\end{array}$} & \multirow{2}{*}{$\begin{array}{c}\begin{array}{c}\text { Inftial } \\
\text { Mass }\end{array} \\
8\end{array}$} & \multirow{2}{*}{$\begin{array}{c}\begin{array}{l}\text { Final } \\
\text { Ylass }\end{array} \\
8\end{array}$} & \multicolumn{2}{|c|}{$\begin{array}{c}\text { Init } 1 \text { la } \\
\text { Vacuum }\end{array}$} & \multirow{2}{*}{$\frac{\begin{array}{c}\text { Yaximun } \\
\text { Temp. }\end{array}}{\mathrm{K}}$} & \multirow{2}{*}{$\frac{\begin{array}{c}\text { Super- } \\
\text { Cool 1ng }\end{array}}{K}$} & \multirow{2}{*}{$\begin{array}{l}\text { Iime on } \\
\text { Yelting } \\
\text { Isotinerm } \\
s\end{array}$} & \multirow{2}{*}{$\begin{array}{c}\begin{array}{c}\text { Iime } \\
\text { Completely } \\
\text { Nolten }\end{array} \\
5\end{array}$} & \multirow{2}{*}{$\begin{array}{l}\text { Tlme on } \\
\text { Freezing } \\
\text { Isotherm } \\
s\end{array}$} & \multirow{2}{*}{$\begin{array}{c}\text { Fe } \\
\text { Fraction } \\
\text { ppb } \\
\text { (by cass) }\end{array}$} \\
\hline & & & $\mathrm{Pa} \times 10^{5}$ & $\operatorname{tor} 5 \times 10^{7}$ & & & & & & \\
\hline $2-3$ & 4.0190 & 3.8425 & 8.0 & 6.0 & 2950 & 0 & 23 & 57 & 18 & $22.9 *$ \\
\hline $2-7$ & 4.0182 & 3.7839 & 7.3 & 5.5 & 2959 & 41 & 33 & 88 & 11 & $26.3 *$ \\
\hline $2-13$ & 3.9378 & 3.8043 & 6.4 & 4.8 & 2936 & 19 & 38 & 6 & 3 & $45.0 *$ \\
\hline $3-21$ & 4.1634 & 4.0684 & 4.0 & 3.0 & 2896 & - & 54 & 0 & - & 1340.2 \\
\hline $3-24$ & 4.2271 & 3.6780 & 8.0 & 6.0 & 3035 & 62 & 18 & 198 & 6 & 350.3 \\
\hline $4-2$ & 4.1542 & 4.0651 & 1.3 & 1.0 & 2989 & 7 & 12 & 8 & 18 & $31.8 *$ \\
\hline $4-9$ & 4.2500 & 4.0665 & 4.8 & 3.6 & 3016 & 114 & 14 & 44 & 18 & $32.9 *$ \\
\hline $4-14$ & 4.2575 & 4.1792 & 2.7 & 2.0 & 2896 & - & 21 & 0 & - & 483.4 \\
\hline $4-16$ & 4.0815 & 3.8979 & 2.7 & 2.0 & 3013 & 0 & 21 & 41 & 21 & 78.0 \\
\hline $5-2$ & 4.1078 & 3.7487 & 4.8 & 4.0 & 2944 & 54 & 51 & 195 & 21 & 20.1 * \\
\hline $5-12$ & 4.0769 & 3.5617 & 7.3 & 5.5 & 2983 & 0 & 36 & 306 & 32 & 175.2 \\
\hline $5-15$ & 4.1294 & 3.5394 & 24.0 & 18.0 & -2950 & 235 & 23 & 309 & 15 & 314.5 \\
\hline $7-1$ & 4.2350 & 3.4388 & 7.3 & 5.5 & 3016 & 0 & 30 & 470 & 15 & $33.7 *$ \\
\hline
\end{tabular}

+Based on melting point of Mo of $2896 \mathrm{~K}$.

*The sensitivity of this chemical analysis (as determined by the blank Fe lerel) 1 s 50 ppb. Hence, these values should be considered as $<50 \mathrm{ppb}$. 
includes the initial and final mass, the maximum temperature while molten, the amount of supercooling before solidification*, the time on the melting isotherm, time completely molten, time on freezing isotherm, and the final $\mathrm{Fe}$ content. The change in mass of the samples has no real meaning due to excessive spitting that occurs initially during melting. Because of the rapid purification rate expected, two experiments (3-21 and 4-14) were performed in which the samples were not quite completely molten (or possibly were just molten) and were promptly solidified. The iron level of these two samples is $1.3 \mathrm{ppm}$ and $483.4 \mathrm{ppb}$ which are the higlest levels of any of the processed samples. Figure 7 shows the Fe content plotted as a function of time completely molten. The results are summarized as follows:

1. Very rapid evaporation of Fe was observed with levels being reduced to $<50 \mathrm{ppb}$ in less than 6 seconds completely molten.

2. Samples which were not completely melted showed Fe levels far below the starting level but above the levels obtained for samples which were completely melted.

3. The Fe content of most of the completely melted samples was beneath the sensitivity of the chemical analysis and, hence, might be much lower than $50 \mathrm{ppb}$. However, these samples showed some evidence of a small but consistent level above the blank which was not correlated with time processed. This may be indicative of contamination after purification.

\footnotetext{
The amount of supercooling is included primarily for information only.
} 
4. Three of eleven experiments completely melted have comparatively high levels of $\mathrm{Fe}$ and it is assumed that contamination of these samples occurred during cooling in the copper dish and handling after purification. Conclusions and Discussion (Part B)

Although Mo with extremely low Fe content has been obtained, a major goal of this year's work was to try to obtain reproducible purification data for the impurity Fe from Mo. This goal has certainly not been met as even a casual glance at Fig. 7 will show. Many precautions have been taken to eliminate scatter in the data. Surface cleaning and sawing was performed carefully. Chemical analysis was performed on bulk samples rather than small samples. Vacuum and temperature during experiments was continuously monitored. However, two facts seem to be causing the difficulty. First, the extremely rapid vaporization of Fe is such that time required for heating to and cooling from the test temperature is larger than times required to purify the sample. It was previously thought that surface depletion of impurity might have greatly slowed the purification process. Second, the extremely low level of $\mathrm{Fe}$ present in most samples makes the effects of handling much more severe. Nevertheless, a few conclusions can be drawn from this data. Metallographic examination of several samples indicate that freezing of the levitated samples occurs from the outside surface to the center. For example, the shrinkage cavity is always in the interior of the sample. Hence, purification ceases for all. practical purposes as soon as solidification starts. Metallographic examination of sample 4-14 shows that the 
sample was completely molten. Since the temperature of this sample did not rise above the melting isotherm even though the power input of the sample was high enough to do so, it is assumed that this sample was just barely completely molten when freezing was initiated. Hence, the Fe content of this sample (483 ppb) will be taken to represent the amount of $\mathrm{Fe}$ left in samples after the melting stage. Two specimens, 2-13 and 4-2, were completely molten for 6 and 8 seconds, respectively, and their final Fe levels were less than $50 \mathrm{ppb}$. So we conclude that the Fe level in completely molten samples is reduced from $483 \mathrm{ppb}$ to at least $50 \mathrm{ppb}$ in 6 seconds or less.

Figure 8 shows the calculated change in Fe level versus time using Eq. (10) and (11) for data representing the experimental configuration. This is very closely approximated by the exponential form of this solution given in the footnote to Eq. (10). Hence it is valid to use this curve to find the time interval to change from $483 \mathrm{ppb}$ to $50 \mathrm{ppb}$. The calculated time for this is 0.54 seconds. So we conclude that at the least, the experimental purification rate is $9 \%$ of the calculated rate. This is considered reasonable agreement for this impurity considering the experimental difficulty of measuring such rapid evaporation rates.

Conclusions about the detailed assumptions of the purification model cannot be made at this point. The assumptions about ideal solution behavior, non-reacting species, and evaporation coefficients cannot be examined based on this data. However, some insight can be gained into the degree of mixing present in R.F. levitated samples. As was described in the introduction, the rate of purification is often limited by transport of impurities to the evaporation surface (i.e., lack of complete mixing) 
rather than by the evaporation process itself. Under these circumstances the surface of the evaporating sample becomes depleted in the impurity and purification is retarded. Because we want to measure purification rates limited by basic evaporation processes and not by transport of impurities in the liquid phase, it is important to assess the degree of mixing in R.F. levitated samples. This is also important for space processing because the case of R.F. stirring is probably the best mixing that can be currently obtained.

The rate of mixing required in a sample to prevent surface depletion of the impurity is a function of the volatility of the impurity. A highly volatile impurity $(\alpha<<1)$ requires more mixing than does a less volatile one $(a<1)$. It has been shown here for the impurity $\mathrm{Fe}$ in Mo $\left(a \sim 10^{-4}\right.$ ), that the purification rate is very rapid. At the slowest the iron content has been reduced by a factor of 10 in less than six seconds. This indicates very rapid circulation times and the probable absence of dead zones in the liquid. Hence, limitations of impurity transport to the evaporation surface are not large. Certainly for less volatile impurities like $\operatorname{Zr}\left(\alpha \sim 10^{-1}\right)$, it can be stated that there will be no measurable limitation to the rate of purification caused by impurity transport in the liquid using levitation melting under the same conditions. Next year's work will measure purification rates for less volatile impurities from Mo and conclusions about the evaporation process itself can be made. 
Acknowledgment

The authors would like to thank I. L. Barnes, R. L. Burke,

B. I. Diamondstone, L. P. Dunstan, E. L.Garner, and E. J. Maienthal of the Analytical Chemistry Division of the National Bureau of Standards for the chemical analysis reported here. 
[1] Paule, R. C., NBSIR 73-402, 33 (1973).

[2] Paule, R. C., Boettinger, W. J., Biancaniello, F.S., NBSIR 74-611, 24 (1974).

[3] Brewer, L. and Searcy, A. W., J. Am. Chem, Soc. 73, 5308 (1951).

[4] Porter, R. F., Schisse1, P., Inghram, M. G., J. Chem. Phys. 23, 339 (1955).

[5] Ackermann, R. J. and Thorn, R. J., J. Am. Chem. Soc. 78, 4169 (1956).

[6] DeMaria, G., Drowart, J. and Inghram, M. G., J. Chem. Phys. 30, 318 (1959).

[7] Drowart, J., DeMaria, G., Burns, R. P., and Inghram, M. G., J. Chem. Phys. 32, 1366 (1960).

[8] Farber, M., Srivastava, R. D., and Uy, 0. M., J. Chem. Phys. 55, 4142 (1971).

[9] Farber, M., Srivastava, R. D., and Uy, O. M., J. Chem. Soc., Faraday Trans. 1, 68, 249 (1972).

[10] Sears, G. W. and Navias, L., J. Chem. Phys. 30, 1111 (1959).

[11] Burns, R. P., Jason, A. J., and Inghram, M. G., J. Chem. Phys. 40, 2739 (1964).

[12] Burns, R. P., J. Chem. Phys. 44, 3307 (1966).

[13] Alcock, C. B. and Peleg, M., Trans. Brit. Cer. Soc. 66, 217 (1967).

[14] Peleg, M. and Alcock, C. B., High Temp. Sci. 6, 52 (1974).

[15] Diamond, J. J. and Schneider, S. J., J. Am. Ceram. Soc. 43, 1 (1960).

[16] Gryvnak, D. A. and Burch, D. E., J. Opt. Soc. Am. 55, 625 (1965).

[17] Mann, J. B., J. Chem. Phys. 46, 1646 (1967).

[18] Paule, R. C., High Temp. Sci. $\underline{6}, 267$ (1974).

[19] JANAF Thermochemical Tables, Dow Chemical Co., Midland, MI (1975). 
[20] Franklin, J. L., et al, Ionization Potentials, Appearance Potentials, and Heat, of Formation of Gaseous Positive Ions, NSRDS-NBS 26 (1969).

[21] Goldsmith, A, Waterman, T. E., and Hirschhorn, H. J., Handbook of Thermophysical Properties of Solid Materials, Vol. 3, pp 36-7, Macmij.1an Company, New York (1961).

[22] Touloukian, Y. S. and DeWitt, D. P., Thermophysical Properties of Matter, Vol. 8, Thermal Radiative Properties Nonmetallic Solids, IFI/Plenum Press, New York-Washington (1972).

[23] Schneider, S. J., Pure \& Applied Chem. 21 [1], 115 (1970).

[24] Brown, F. E., Trans. Chem. Soc. 35, 550 (1879).

[25] Lord Rayleigh, Phil. Mag. ㅇ, 534 (1904).

[26] Obradovic, N. D. and Bennett, G. H. J., J. Inst. Metals 97, 186 (1969).

55 


$$
\text { , }
$$




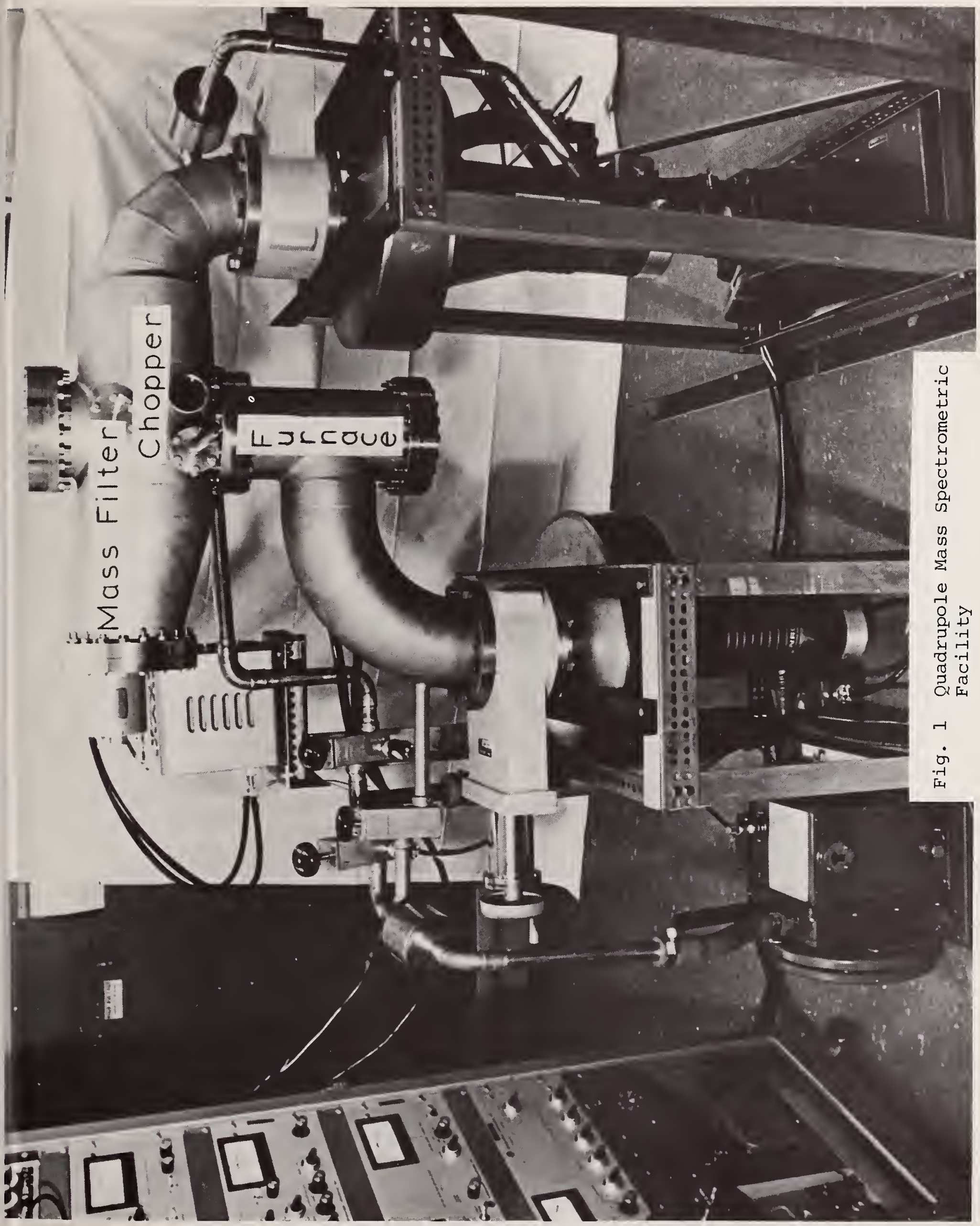





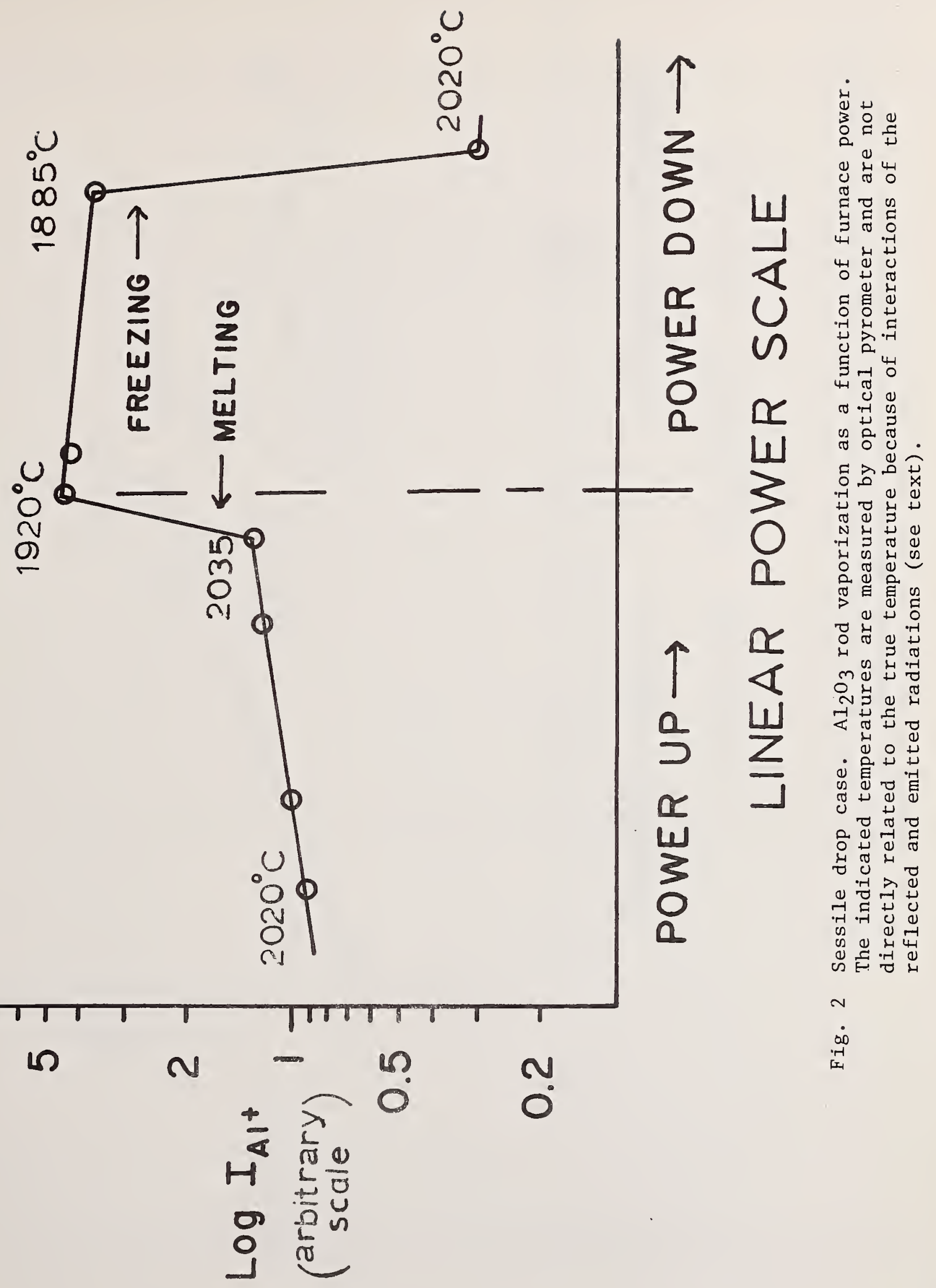





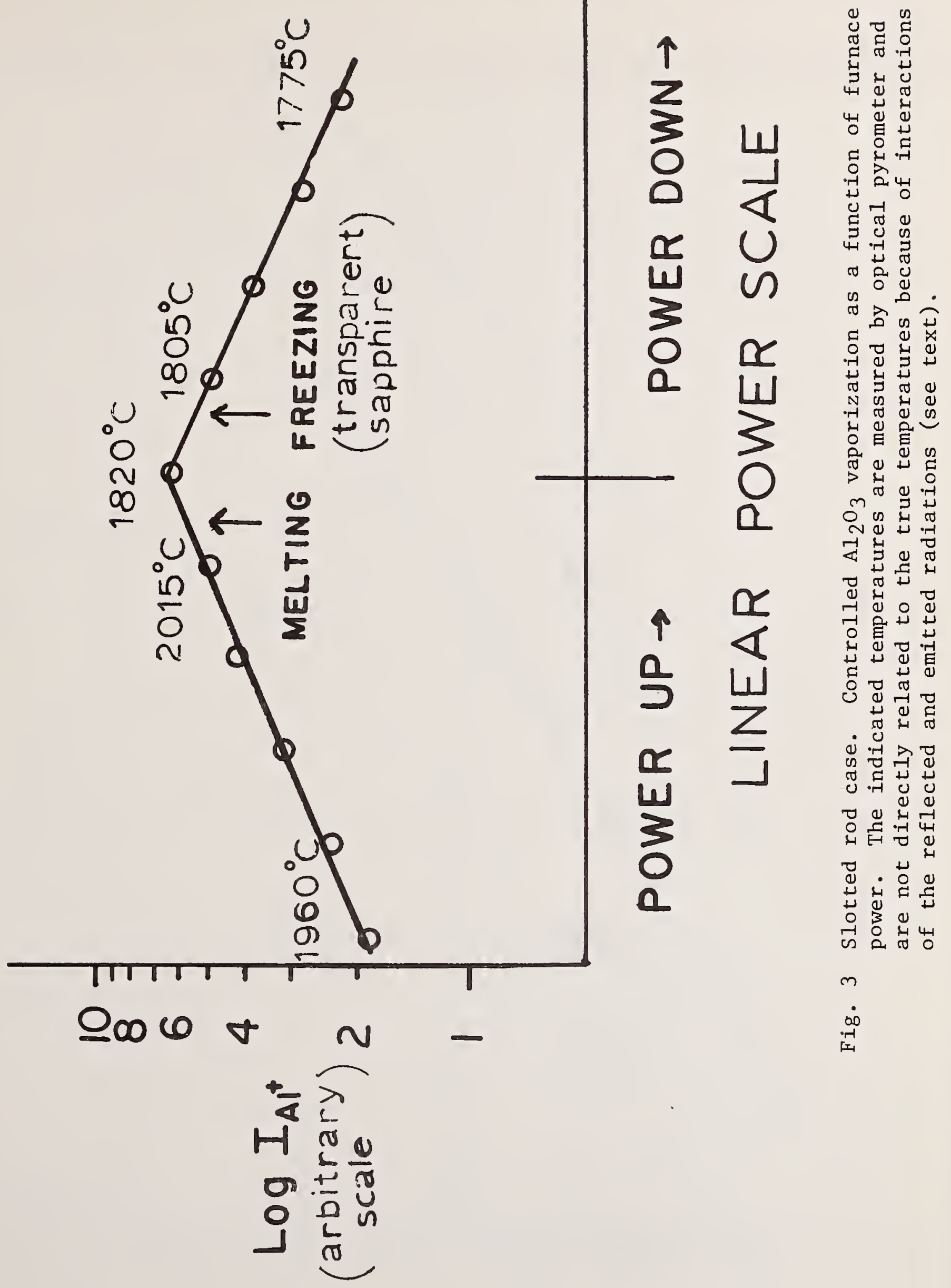





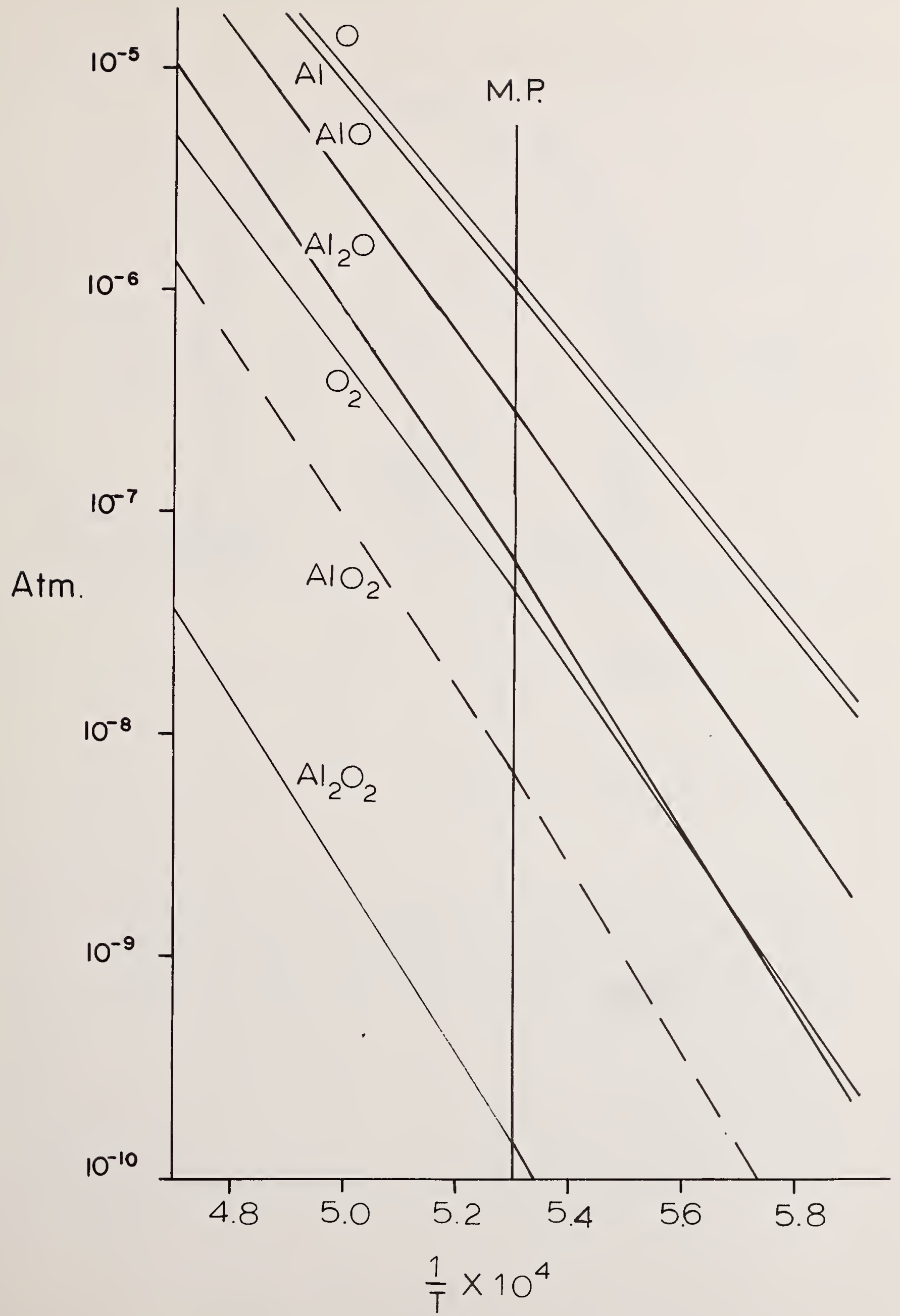
Fig. $4 \mathrm{Al}_{2} \mathrm{O}_{3}$ Equilibrium Vaporization
Pressures into Vacuum 



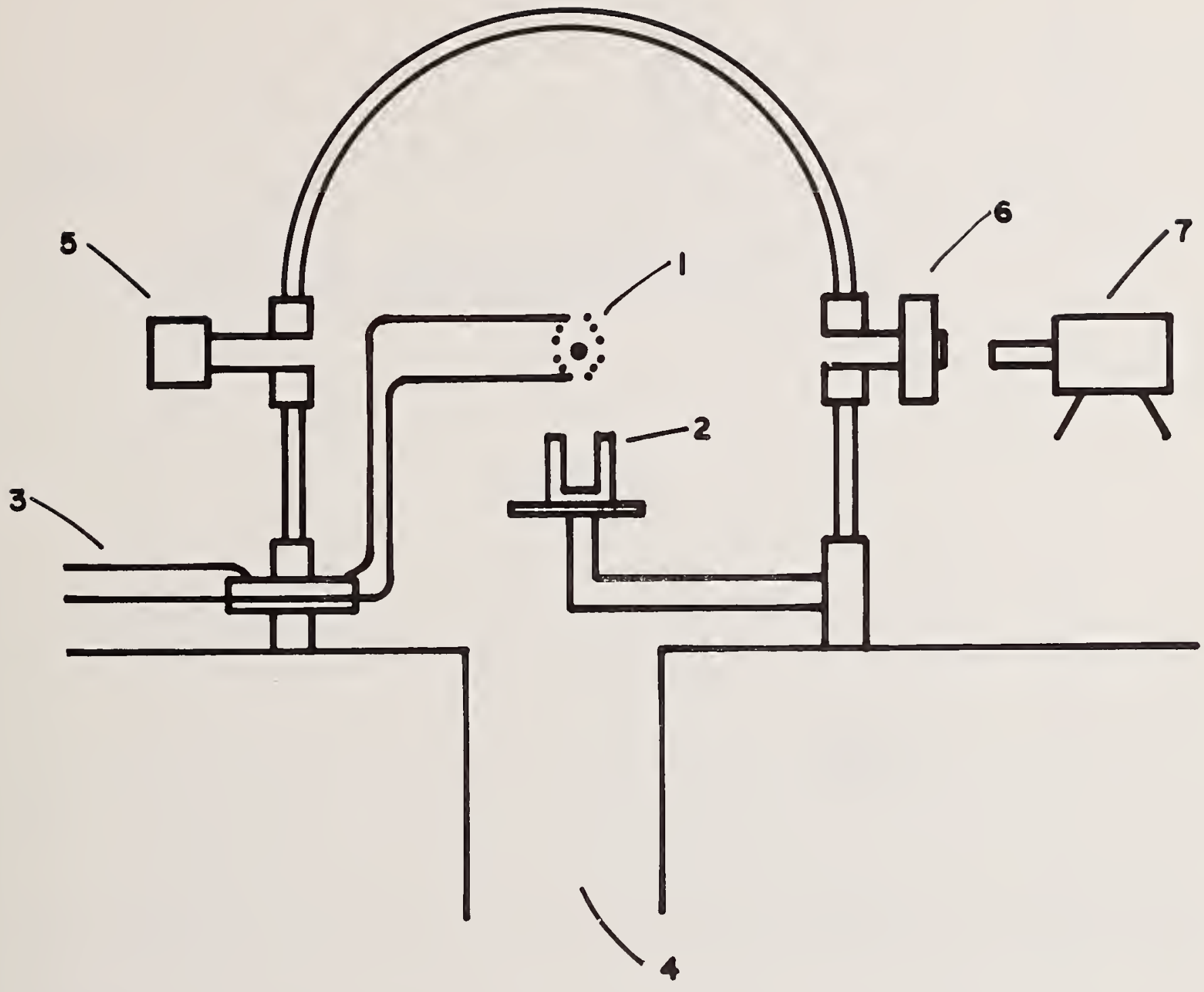

Fig. 5. Schematic of the levitation melting facility. 1) Sample and levitation coil; 2) copper cup to catch solidified sample; 3) R.F. leads to load-coil transformer and generator; 4) to vacuum system; 5) ionization gauge; 6) window to prevent accumulation of metallic vapor; 7) automatic optical pyrometer. 



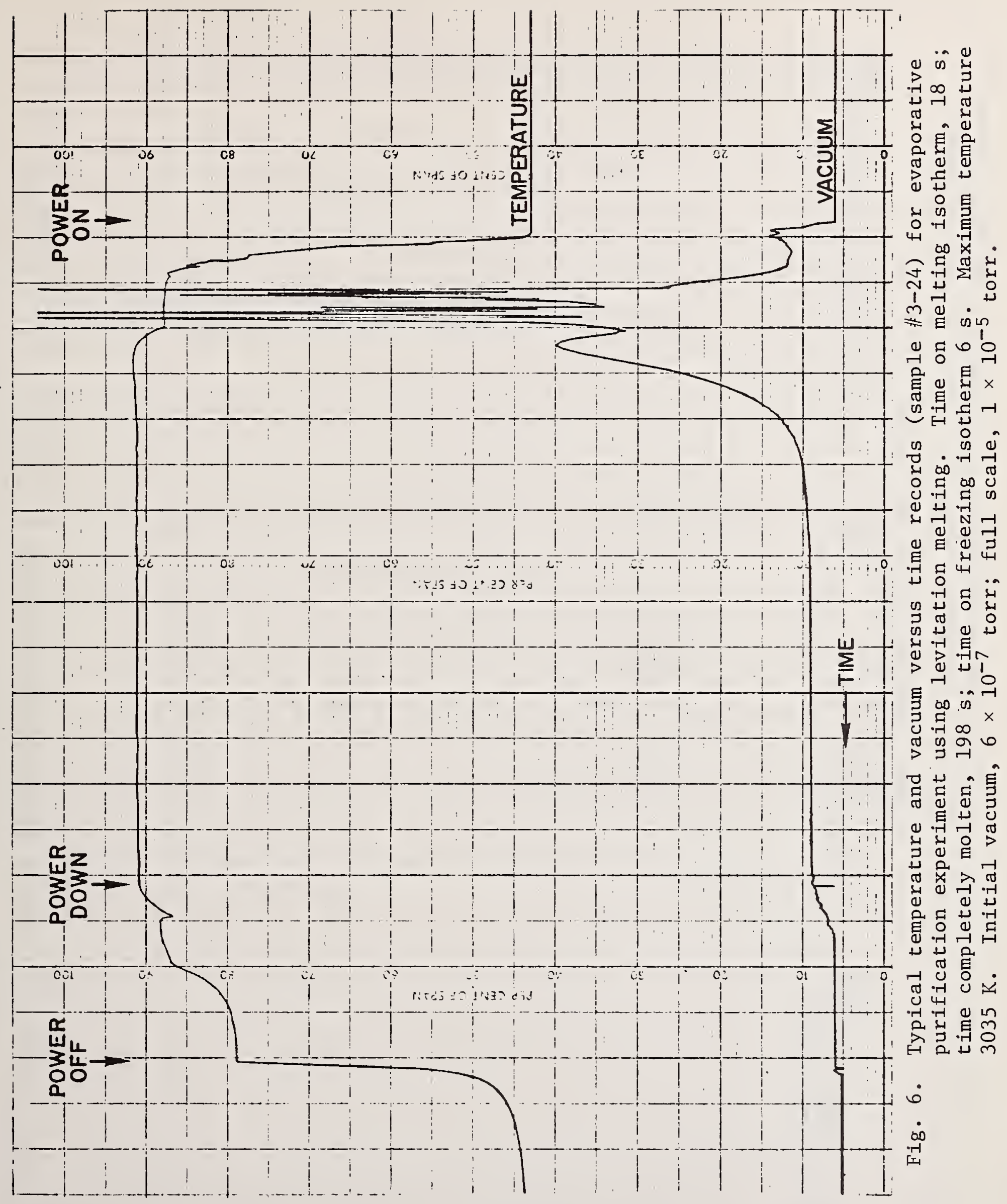





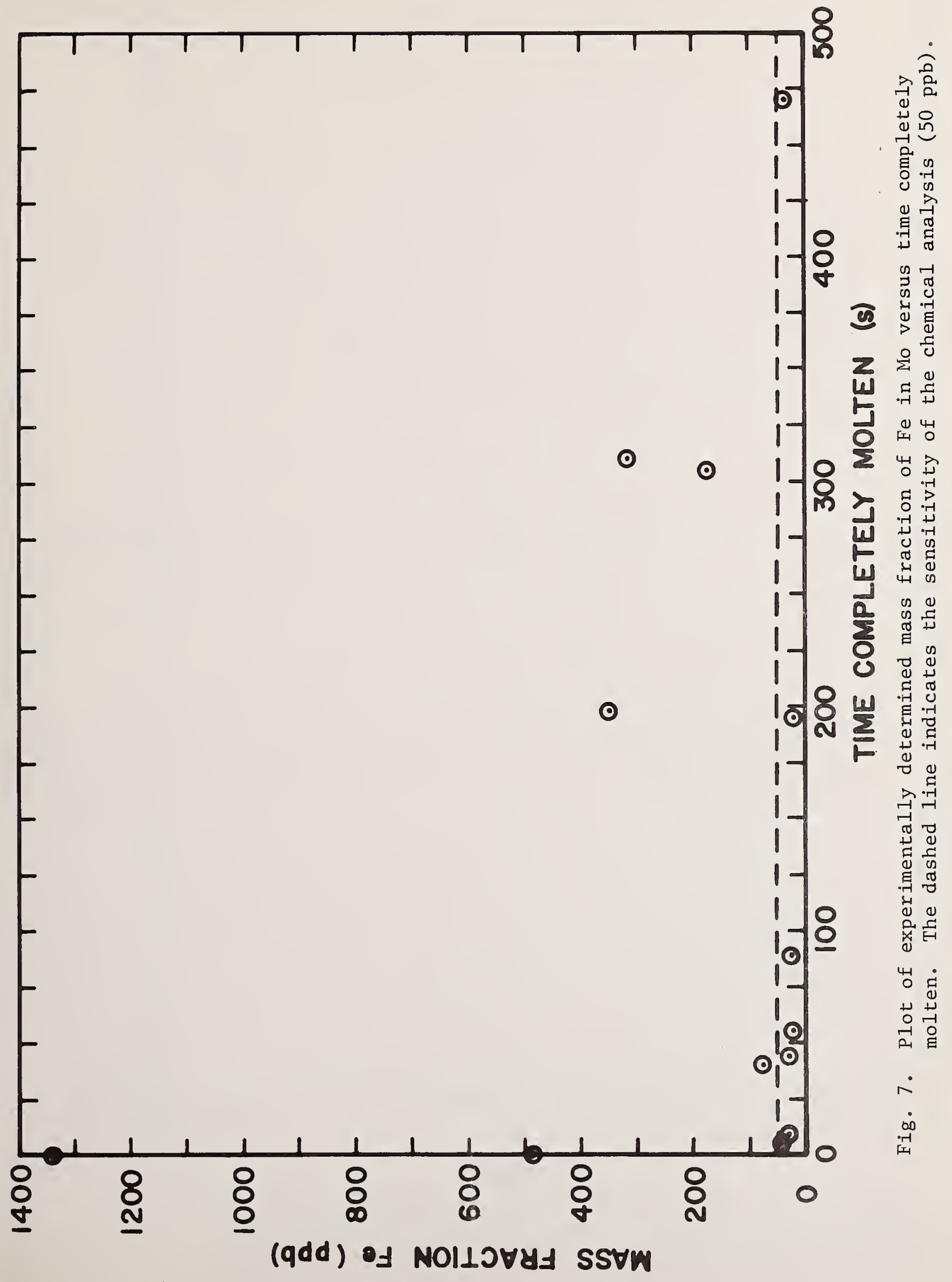





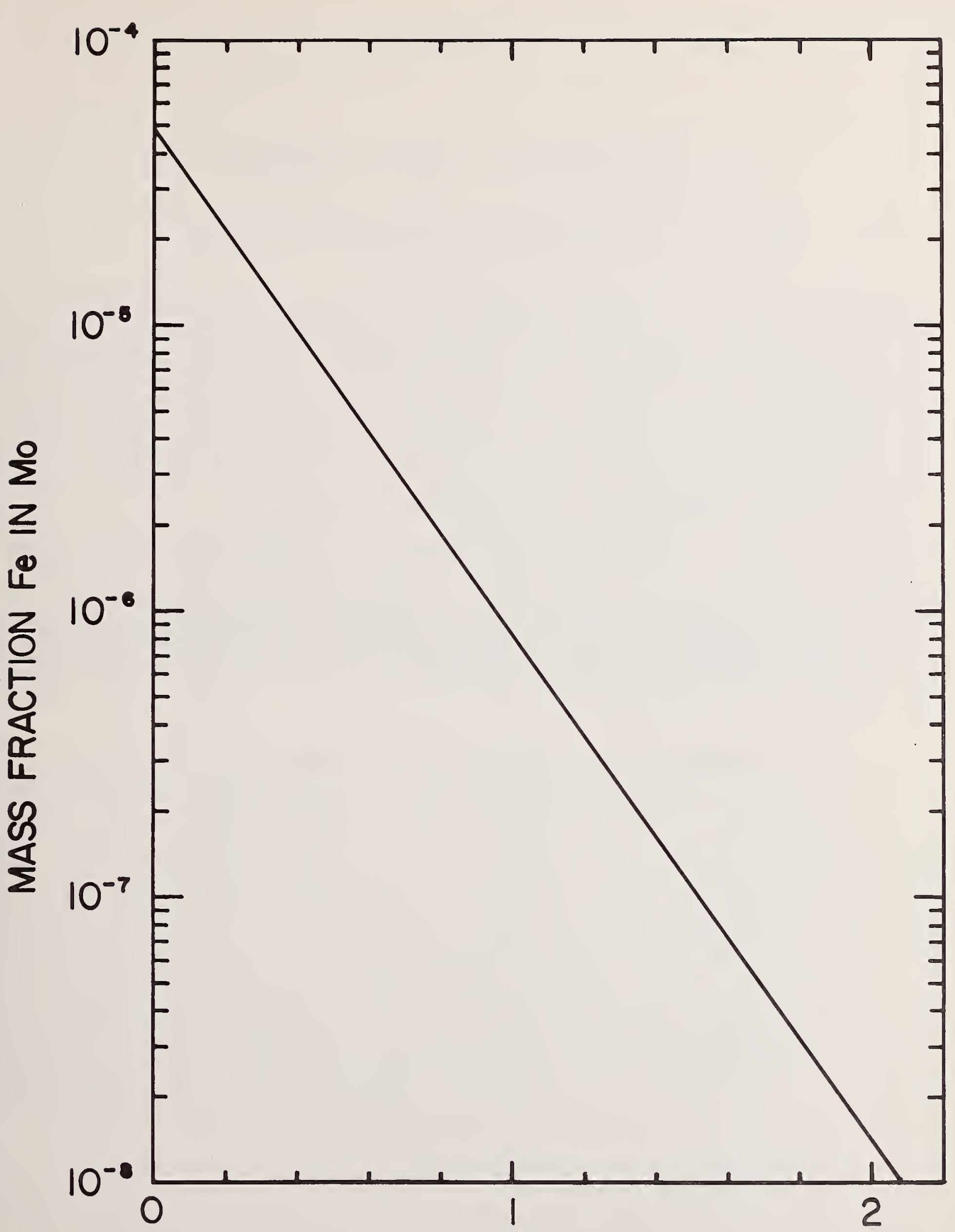

TIME (s)

Fig. 8. Plot of calculated rate of purification for a $4 \mathrm{~g}$ Mo sample containing $48.4 \mathrm{ppm} \mathrm{Fe}$ (by mass) at $3000 \mathrm{~K}$. Based on remaining number of moles calculated using Eqs.10 \& 11 with $\mathrm{P}_{\mathrm{Mo}}=$ $1.002 \times 10^{-4}$ atm., $\mathrm{p}_{\mathrm{Fe}}=0.521 \mathrm{~atm} ., \mathrm{A}=3.0484 \mathrm{~cm}^{2}, \quad(\alpha=$ $1.4674 \times 10^{-4}$ ). 
Determination of Iron in Molybdenum

Method: Extraction-spectrophotometric determination of iron as the Fe(II)-Bathophonanthroline complex.

Procedure: The molybdenum disks were cut in half to obtain samples weighing 0.7-1.l g. These samples were etched with dilute hydrochlor:ic acid to remove any surface iron contamination. Subsequentiy, the samples were dissolved in 100-ml Teflon beakers using a nitric acid/sulfuric acid mixturc. The nitric acid was removed by adcition of hydrochloric acid followed by preconcentration. After acicition of appropriate masking agents and adjustment of $\mathrm{pH}$, the samples were dilutca to volume. Duplicate aliquots were taken for spectrophotometric analysis, using Bathophenanthroline as the chromogenic reagent and l-hexanol as the extraction solvert.

NBS high purity, sub-boiling acids were used throughout this study and al.i reagents, (i.e. ammonium tartrate, ascorbic acid, hydroxylamine hydrochloride, boric acid, and Bathophenanthroline) wore freed of trace iron contamination by repeated extractions with Bathophenanthroline-hexanol. To minimize the environmental blark, all sanple dissolutions were performed in the clean room facilities of the Analytical Chemistry Division.

\begin{tabular}{rcc} 
Sample & Weight, $g$ & Iron, ppm \\
\cline { 2 - 2 } & 0.7 & $54.0 ; 54.2$ \\
B & 1.0 & $46.9 ; 47.5$ \\
C & 1.1 & $43.8 ; 43.9$ \\
$2-3$ & 0.7 & $<2$ \\
$2-7$ & 1.0 & $<2$ \\
$2-13$ & 0.8 & $<2$ \\
$3-2]$ & 1.1 & $<2$
\end{tabular}


Appendix II

Iron Concentration of High Purity Molybdenum Metal

by

Isotope Dilution Mass Spectrometry

The concentration of iron in high purity molybdenum metal was determined by isotope dilution mass spectrometry. Samples ranging in weight from $0.8 \mathrm{~g}$ to $1.6 \mathrm{~g}$ were etched in $\mathrm{HNO}_{3}-\mathrm{H}_{2} \mathrm{SO}_{4}$ and then $\mathrm{HCl}$ to remove surface contamination. The samples were weighed, spiked with ${ }^{5} \mathrm{Fe}$, dissolved in a $\mathrm{HNO}_{3}-\mathrm{H}_{2} \mathrm{SO}_{4}-\mathrm{H}_{2} \mathrm{O}$ solution, and evaporated to dryness. The residue was taken into solution using $\mathrm{NH}_{4} \mathrm{OH}$ and the solution again evaporated to dryness. The iron was then separated from its matrix using anion exchange chromatography. Reagents purified by sub-boiling distillation were used throughout the analysis.

A triple filament rhenium ribbon ion source was used for mass spectrometric analysis. $\mathrm{FeCl}_{3}$ in nitric acid solution was evaporated to dryness on each sample filament and then partically reduced in a hydrogen atmosphere to a mixture of iron oxides and metallic iron.

The results of the iron concentration determination and the analytical blank determination are shown in Tables 1 and 2 , respectively. Columi 4 of Table 1 shows the ratio of the total iron (sample + blank) to the average blank. Since the iron concentration of most of the samples is less than $100 \mathrm{ng}$ and yields total iron to blank ratios $<3$, it is recommended that an upper limit statement be used for these samples. For example, it is recommended that samples $1-5,7$, and 15 be assigned a value $<50 \mathrm{ng}$. The remaining samples have total iron to blank ratios which are sufficiently large to quote the values reported in Table 1 . For these samples $(6,8-12)$, the estimated accuracy of $\pm 30 \mathrm{ng}$ is the sum of the error terms due to isotopic ratio measurement, spike calibration, variability of the analytical blank and ${ }^{56} \mathrm{Fe}$ background contribution from the ionizing filament. 
Table 1. Concentration of Iron in High Purity Molybdenum Metal. Sample No. ng/g of $\mathrm{Fe}^{\mathrm{a}}$ Total $\mathrm{Fe}$ (umols) Total Fe/Blank
(1) $2-3$
$22.9^{b}$
0.001095
2.4
(2) $4-9$
$32.9^{\mathrm{b}}$
0.0009862
2.2
(3) $4-2$
$31.8^{\mathrm{b}}$
0.0009064
2.0
(4) $2-7$
$26.3^{\mathrm{b}}$
0.001158
2.5
(5) $5-2$
$20.1^{b}$
0.0008607
1.9
(6) $5-12$
175.2
0.002908
6.4
(7) $2-13$
$45.0^{\mathrm{b}}$
0.001433
3.1
(8) $5-15$
314.5
0.006468
14.2
(9) $4-14$
483.4
0.011767
25.8
(10) $4-16$
78.0
0.002100
4.6
(11) $3-21$
1340.2
0.039728
87.2
(12) $3-24$
350.3
0.009944
21.8
(13) $7-1$
$33.7^{\mathrm{b}}$
0.001394
3.1

a Corrected for blank contribution of $0.0004554 \mu \mathrm{mols}$ (25.4 ng). b Upper limit value of $<50 \mathrm{ng}$ recommended. 
Table 2. Iron Analytical Blank Determinations.

Blank No.

1

2

3

4

5

6 ng

19.96

22.07

24.15

24.56

22.21

39.53

Average $=25.4$ $\mu \mathrm{mols}$

0.0003574

0.0003952

0.0004342

0.0004399

0.0003978

0.0007080

0.0004554 


\title{
Task 3
}

Vacuum Effects in the Preparation of

Composite Materials

Harvey Yakowitz

Metallurgy Division

Institute for Materials Research

\begin{abstract}
Summary
A method for determining the description in-depth of $X$-rays within a material has been deduced. This method is applicable to composite materials both conventional and laminates. The method is described in detail along with examples. Other analytical tools for composites such as energy dispersive analysis are described. A brief description of the computer program for carrying out these analyses is given. Some specific results on SiC-Al matrix composites supplied by Marshall Space Flight Center are included.
\end{abstract}


Introduction

In order to characterize composite materials by means of electron beam produced $\mathrm{X}$-rays, the distribution in depth of these $\mathrm{X}$-rays must be determined. This distribution can be obtained experimentally but only with extreme difficulty; the experimental errors are potentially very large. Attempts to fit the distribution by means of Monte Carlo techniques can be carried out but this method is cumbersome, expensive, and not particularly sensitive. This report will describe for the first time a simple analytical expression describing this distribution function. Furthermore, the way in which the function can be used to analyze layered structures or film composites and composites of cylindrical or spherical particles embedded in a matrix will be given in detail.

Since many such analyses must be carried out with energy dispersive $\mathrm{X}$-ray microanalysis, this technique will be outlined briefly. Special emphasis will be placed on characterization of the continuum X-ray background produced during energy dispersive X-ray microanalysis. A computer data reduction scheme incorporating this continuum background correction will be described briefly as well.

Some further studies on the SiC filaments in Al matrix composite provided by Marshall Space Flight Center will be discussed. Specifically, the number and distribution of phases will be considered.

Finally, some by-products of the derivation of the X-ray distribution function will be reported. These include a new absorption effect correction and atomic number correction for conventional X-ray microanalysis. These new correction formulae can be used not only in the analysis of composite materials but also are applicable to general X-ray microanalysis. 
Quantitative evaluation of thin film specimens, either unsupported or upon a substrate, poses difficulties for the X-ray microanalyst. The chief problem is that the electron beam is not completely stopped within the thin film so that conventional quantitation schemes cannot be safely applied. The purpose of this report is to describe the difficulties encountered in thin film analysis in detail and to propose a simple method for obtaining quantitative information from thin film specimens.

Nature of the Problem

An electron striking a thin unsupported film has three possible interactions: backscatter, transmission or total energy loss within the film, i.e., absorption. If the film is supported on a substrate, the situation is more complex. The possibilities for this case are shown schematically in Fig. (1). Any method for evaluating thin films must account for reductions in $\mathrm{X}$-ray emission due to electron transmission and interactive effects due to the substrate, if any. The analyst usually measures intensity ratios, i.e., the intensity from the thin film constituents with respect to bulk standards. Therefore, what is needed is a means to predict this intensity ratio as a function of sample thickness and composition -- both of which are usually unknown. In addition, the method should also be applicable for non-normal electron beam incidence since many scanning electron microscopes (SEM's) require the specimen to be tilted for X-ay analysis.

There have been a number of approaches taken toward obtaining a thin film analytical procedure. For the unsupported specimen, there is 
1ittle electron-solid interaction so that the X-rays produced escape the specimen after suffering negligible absorption and fluorescence effects. Then observed intensity and concentration are linearly related. A number of investigators have proposed techniques for determining the form of equations taking advantage of this property of thin-film, X-ray interactions [1]-[6]. These equations range from empirical relations in which the proportionality factor is determined from intermediate standards to complex theoretical schemes requiring fairly extensive computation facilities. For thin films mounted on substrates, two distinct approaches have been taken. The first consists of attempting to determine the number of ionizations produced by each group of electrons which follow any of the paths delineated in Fig. (1). These contributions are summed to obtain the total number of ionizations in the film; the cotal number of ionizations from a bulk standard can be obtained [7]. The ratio of these ionizations will be the observed intensity ratio. This method requires knowledge of the $X$-ray range, the backscatter correction factor, $R$, the ionization cross-section, Q, and the electron stopping power, S. Each of these parameters is used in conventional electron probe microanalysis, and each has been reviewed in detail in terms of derivation and uncertainties [8]. A further assumption of this method is that the substrate does not contain any of the constituents found in the film. The final result is a set of integral equations which are solved by normal equation theory using matrix methods [9], [10]. Hence, fairly extensive computation facilities are needed and the method is subject to all of the uncertainties associated with conventional X-ray microanalysis. Nevertheless, this approach has proved valuable in practice and is in current use $[9],[10]$. 
The other approach is to attempt to determine the number of $\mathrm{X}$-rays generated as a function of depth in the specimen and in the standard. The distribution in depth of $\mathrm{X}$-ray generation is commonly referred to as $\phi(z)$, with $z$ being the mass thickness of the specimen, following the concept introduced by Castaing [11]. A plot of $\phi(z)$ against $z$ is shown in Fig. (2); normal electron beam incidence was used [12]. The shape of this $\phi(z)$ plot is typical of all elements bombarded with electrons at normal incidence. In effect, $\phi(z)$ represents the number of X-rays generated at depth $z$ with respect to the number of X-rays generated from a "free surface" of the same material, i.e., a layer of vanishingly small thickness. The peak occurs because electrons penetrate some distance before producing ionizations and because electrons scatter back toward the surface. If the specimen is tilted, the $\phi(z)$ curves change shape since the electrons remain nearer the surface; the effect has been studied by means of Monte Carlo simulations [13]. Figure (3) shows some results for a copper target.

The great value of the $\phi(z)$ curve is that the integral of the curve yields the total number of X-ray photons generated per incident electron. Knowing this, one need only correct for absorption and fluorescence effects in order to obtain the number of emitted X-rays from the specimen. For thin film analysis, the $\phi(z)$ curve for the thin film is needed as well as that for the standard. If a bulk pure element is used as the standard, the only correction needed in principle is for absorption; fluorescence effects are nonexistent in the standard and negligible in the unknown. 
Relatively few $\phi(z)$ curves have been determined experimentally; the experiment is tedious and difficult [11] - [15]. Attempts have been made to derive expressions enabling $\phi(z)$ to be calculated. Analytical expressions have been discussed by Criss and Birks [16] and by Reuter [17]. Many calculated $\phi(z)$ curves have been obtained by Monte Carlo methods [13], [18], [19]. The $\phi(z)$ approach has been applied to thin film analysis both from the point of view of measuring the thickness of pure element films [17], [20]-[22] and actual analysis [22]. In this report, we describe a simple method which can be used to obtain $\phi(z)$ for bulk specimens with any electron beam incidence angle; a procedure based on this calculated $\phi(z)$ to obtain quantitative thin film analysis will then be described.

Thin Film Analysis with $\phi(z)$ Data

In principle, one needs only to obtain the $\phi(z)$ curves for the specimen and standard. For the thin film specimen, the $\phi(z)$ curve is affected by the nature of the substrate or lack thereof. Hence, the $\phi(z)$ curve for the thin film case will depend upon the electron scattering properties of the substrate; when these factors are taken into account, an appropriate $\phi(z)$ relation can be obtained for the thin film.

In order to obtain the $X$-ray intensity emitted by the standard, the integral of $\phi(z)$ for the standard over the range of $z$ from 0 to the $X$-ray range, $z_{r}$, must be corrected for absorption in the bulk specimen [8]. The ratio of the two integrals gives the expected intensity ratio, $k_{r}$, for a film of thickness $z$ with respect to a pure bulk standard. Thus, one can prepare a curve of $k_{r}$ versus film thickness for pure element films. 
In practice then, one requires a means to formulate $\phi(z)$ for the bulk standard as a function of incident electron beam energy, incidence angle, and atomic number as well as a means to obtain the integral of this expression. Furthermore, in order to obtain the required information from the film, the effect of the substrate must be taken into account including the case where the atomic number of the substrate, $\mathrm{Z}_{\mathrm{SS}}$, is zero.

Formulation of $\phi(z)$ for Bulk Material

Theoretically, the LaPlace transform of the absorption correction yields the $\phi(z)$ function equation in electron probe microanalysis. Recently, direct investigation of the absorption effect resulted in a proposed relation for the absorption correction factor, $f(x)$, such that

$$
\frac{1}{f(x)}=\left(1+1.2 \times 10^{-6} x y\right)^{2}
$$

where $x=\frac{\mu}{\rho} \csc \psi, \frac{\mu}{\rho}$ is the $X$-ray mass attenuation coefficient, $\psi$ is the $X$-ray emergence angle from the specimen and $\gamma=E_{0}^{1.65}-E_{q}^{1.65}$, where $E_{o}$ is the incident electron beam energy and $E_{q}$ is the critical excitation potential for the electron shell of interest, viz., $q=K, L$, or $M$ [23]. The inverse LaPlace transformation of Eqn (1) should yield a valid expression of $\phi(z)$. However, this procedure does not provide an entirely satisfactory result because the value of $\phi(z)$ when $z$ is zero, called $\phi_{0}$, is zero. All experimental determinations of $\phi_{0}$ indicate that this quantity is, in fact, greater than unity [11],[12], [14],[15]. Furthermore the effect of varying the electron beam incidence angle on $\phi(z)$ derived in this way is not at all clear. 
In view of this state of affairs, we examined both experimental and Monte Carlo generated $\phi(z)$ curves in the hope that a rational and consistent analytical $\phi(z)$ formulation could be inferred. Consider Fig. (4) In which the major features of a typical normal-incidence $\phi(z)$ curve are noted. Our observations based on all such $\phi(z)$ results we could obtain are that the function increases parabolically from $\phi_{0}$ through the peak at $(h, k)$, continues to follow the same parabola to about a $z$ value of $1.5 \mathrm{~h}$ and then drops off exponentially to the $\mathrm{X}$-ray range, ${ }_{\mathrm{r}}{ }^{\cdot}$ In particular, for the region of $0 \leq z \leq 1.5 \mathrm{~h}$

$$
\phi(z)_{z \leq 1.5 h} \simeq h^{-2}(z-h)^{2}\left(\phi_{0}-k\right)+k
$$

while for the region $1.5 \mathrm{~h} \leq \mathrm{z} \leq \mathrm{z}_{\mathrm{r}}$

$\phi(z)_{z \geq 1.5 h}=0.25\left(\phi_{0}+3 k\right)\left\{\exp \left[3 h \sec \theta /\left(z_{r}-1.5 h\right)\right]\right\}\left\{\exp -\left[2 z \sec \theta /\left(z_{r}-1.5 h\right)\right]\right\}$

where $\theta$ is the electron beam incidence angle. The major problems were to obtain $\phi_{0}, h, k$ and $z_{r}$ as a function of incident beam energy, electron shell and atomic number.

Reuter has discussed $\phi_{0}$ in detail and following Duncumb and Melford formulated an expression [17] as

$$
\phi_{0}=1+2.8 n(1-0.9 W)
$$

where $n$ is the backscattering coefficient for electrons which has been measured over a wide range of conditions [24] - [26] and $W$ is $E_{q} / E_{0}$. One can fit $n$ as a function of atomic number, $z$, with a polynomial.

The X-ray range, $z_{r}$, has been investigated by a number of authors [11], [27] - [30]. Figure (5) shows that results from these expressions may 
vary considerably with respect to one another. Hence, apparently there will be an uncertainty introduced depending upon which expression for ${ }_{r}$ is chosen. When our results were compared with experimental findings, Heinrich's expression for $z_{r}$ seemed to compare best:

$$
z_{r}=0.007\left(E_{o}^{1.65}-E_{q}^{1.65}\right)\left(\mathrm{mg} / \mathrm{cm}^{2}\right)
$$

However, we are by no means certain that Eqn (4) is completely accurate.

The next task was to determine the values $h$ and $k$. Examination of the available evidence for normal electron beam incidence indicated that the ratio $\left(\mathrm{h} / \mathrm{z}_{\mathrm{r}}\right)$ was a decreasing function of atomic number and virtually independent of beam energy. Apparently, the peak in the $\phi(z)$ curve moves deeper into the material as a function of the parameter $\left(E_{0}^{1.65}-E_{q}^{1.65}\right)$, $E$ values in $\mathrm{keV}$, while the backscattering propensity of the material $n$ opposes this tendency. Thus, the ratio $\mathrm{h} / \mathrm{z}_{\mathrm{r}}$ could be expressed in terms of either $n$ or for normal beam incidence, atomic number $z$, since $n$ depends only weakly on beam energy:

$$
\begin{aligned}
& \mathrm{h} / \mathrm{z}_{\mathrm{r}}=0.487-1.60 n+2.44 n^{2}-1.33 n^{3} \\
& \mathrm{~h} / \mathrm{z}_{\mathrm{r}}=\mathrm{z}^{-1 / 2}+10^{-5} \mathrm{z}^{2}
\end{aligned}
$$

Equation ( $5 a$ ) may be used with any electron beam incidence angle while Eqn (5b) may only be used in the normal incidence case. The value of $n$ increases as a function of electron beam incidence angle, approaching unity at grazing incidence [26]. If $n$ in Eqn (5a) approaches unity then $h$ tends to zero in accord with Fig. (3).

Finally, the value of $k$ was estimated by fitting available data to the relation 


$$
k=\phi_{0}(I+0.35 \cos \theta \ln U)
$$

where $U=E_{0} / E_{q}$ and $\theta$ is the electron beam incidence angle. While the reasons for the exact form of the multiplier for $\phi_{0}$ in Eqn (6) are not readily apparent, the dependence on a slowly varying function of $U$ is not altogether surprising. As $U$ increases, the depth at which the peak occurs increases, i.e., $h$ increases. But, the average electron is steadily losing energy as it proceeds along its path. Apparently then, the number of ionizations produced at depth $h$ follows a roughly similar relation as does the ionization cross-section [31] which also increases with In U. Results from Eqn (2) are shown in Figs. (2) and (3) for comparison purposes; the agreement is typical of many such tests.

The integration of Eqn (2) is straightforward; the result for the intensity generated per incident electron, $I_{G}$, is

$$
I_{G}=\int_{0}^{z} \phi(z) d(z)=0.125\left(\phi_{0}+3 k\right)\left(Q_{0}+3 h\right)
$$

with $Q_{0}=\left(z_{r}-1.5 h\right)\left(1-e^{-2}\right)=0.865\left(z_{r}-1.5 h\right)$

The appropriate $\phi(z)$ relation for the thin film specimen and its integral are now needed.

The $\phi(z)$ Relation for a Thin Film Specimen

The thin film specimen has an effective backscattering coefficient, $\eta_{E^{*}}$ If there is no substrate, then $n_{E}$ is the backscattering coefficient for the film, $n_{B F}$. If there is a substrate then

$$
n_{E} \simeq n_{B F}+n_{S S} n_{T F}\left(1-n_{B F}\right)
$$


where $\eta_{\text {SS }}$ is the backscattering coefficient for the bulk substrate having atomic number $z_{S S}$, and $n_{T F}$ is the fraction of electrons transmitted through the thin film. We have been able to fit transmission and backscattering data available as follows:

$$
\eta_{B F} \simeq 4 n_{Z}\left(z / R_{K-0}\right)
$$

where $\eta_{z}$ is the bulk backscattering coefficient for atomic number $z, z$ is the film thickness in $\mathrm{mg} / \mathrm{cm}^{2}$ and $\mathrm{R}$ is the maximum electron range, in $\mathrm{mg} / \mathrm{cm}^{2}$, of Kanaya and Okayama [32]:

$$
\mathrm{R}_{\mathrm{K}-0}=2.76 \times 10^{-3} \mathrm{E}_{0}^{5 / 3} \mathrm{~A} / \mathrm{Z}^{8 / 9}
$$

A being the atomic weight of the target.

For $n_{\mathrm{TF}}$ we obtained

$$
n_{\mathrm{TF}}=\exp -\left\{\left(0.03 \times 2 \times 10^{-4} \mathrm{E}_{\mathrm{O}}\right) \mathrm{Z}\left(\mathrm{z} / \mathrm{R}_{\mathrm{K}-0}\right)\left[1+4\left(\mathrm{z} / \mathrm{R}_{\mathrm{K}-0}\right)\right]\right\}
$$

These expressions differ in form to some extent from those suggested by Reuter [17], but the results are similar.

To obtain $\phi(z)$ for the thin film specimen, one needs to substitute ${ }^{n_{E}}$ into Eqns (3) and (5a). Then the values of $\phi_{0}, h$ and $k$ for the thin film specimen, called $\phi_{0}^{+}, h^{+}$and $k^{+}$for the remainder of the discussion, can be obtained. The form of the $\phi(z)^{+}$curve is assumed to be as given in Eqn (2). The integral relationships are:

For the region $0 \leq z \leq 1.5 \mathrm{~h}^{+}$

$$
\int_{0}^{z<1.5 h^{+}} \phi^{+}(z) d(z)=\frac{z\left(\phi_{0}^{+}-k^{+}\right)}{h^{+2}}\left[\frac{z^{2}}{3}-h^{+} z+h^{+2}\right]+k^{+} z
$$


and for the region $1.5 h^{+} \leq z \leq z_{r}$

$$
I_{G}^{+}=\int_{0}^{z \geq 1.5 h^{+}} \phi^{+}(z) d(z)=\left(\phi_{0}^{+}+3 k^{+}\right)\left(3 h^{+}+Q\right) 0.125
$$

where $Q=\left(z_{r}-1.5 h^{+}\right)\left\{\left[1-\exp -\left[\left(2 z-3 h^{+}\right) /\left(z_{r}-1.5 h^{+}\right)\right]\right\}\right.$

The required ratio of photons generated in the thin film specimen to those generated in the standard can be obtained by dividing Eqn (12) or Eqn (13) by Eqn (7) depending on whether $z \geq 1.5 \mathrm{~h}^{+}$or not. Figure (4) shows $\phi(z)$ for bulk $\mathrm{Au}$ and for $\mathrm{Au}$ films on $\mathrm{Al}_{2} \mathrm{O}_{3}$ as calculated from Eqns (2) and (12).

But the value of $k_{r}$ measured is not $I_{G}^{+} / I_{G}$ but rather

$$
k_{r}=\frac{I_{G}^{+}}{I_{G}} \frac{f^{+}(x)}{f(x)}
$$

The value of $f(x)$ can be obtained from Eqn (1). For many thin film specimens $f^{+}(X)$ is virtually unity. We have assumed that $f^{+}(x)$ varies linearly with specimen thickness, being unity at $z=0$ and being $f(x)$ at $z=z_{r}:$

$$
f^{+}(x)=1+\left[\left(\frac{z}{z_{Y}}\right)(f(x)-1)\right]
$$

The required computations for obtaining $k_{r}$ as a function of specimen thickness for a pure element can be carried out with the aid of a desk calculator. We have also written a short (40 statements) computer program in the BASIC language to obtain this function.

Comparison of Results with Experimental Evidence

Several investigators have reported results of measurement of film thickness as a function of X-ray intensity; parameters such as incident 
beam energy and substrate material were varied as well [17], [20]-[22] Tables I-III give results of the method proposed in this report as compared with experimental results obtained by Reuter [17], and by Bishop and Poole [20]. Figure (6) shows a comparison between results calculated by our method and Monte Carlo and experimental results reported by Kyser and Murata [22]. Additional results for gold films on various substrates were reported by Bolon and Lifshin [20]. These workers utilized the Au-Ma line in conjunction with an instrument having an X-ray emergence angle of $18^{\circ}$. Therefore, the absorption correction factor for the pure element standard was about 2.5. Uncertainties in the absorption effect in this case make comparison of these experimental values with results from our model ambiguous as indicated in Fig. (7)..

The thin film procedure we propose reproduces, within about $\pm 10 \%$ relative, experimental data taken under a wide variety of conditions of incident beam energy, specimen thickness, and substrate material. But these comparisons are only for materials of known composition. The effect of introducing the complication of unknown composition as well as unknown thickness still requires consideration.

\section{Compositional Analysis of Thin Film Specimens}

Presumably, the investigator measures the emitted intensity for element $i$ from the thin film specimen, $I_{i, E}^{+}$, and the emitted intensity from a bulk specimen. We will assume that the intensity from bulk pure " $i "$ is $I_{i, E}$ since one can obtain this quantity even if multielement standards [33] or calculational schemes for pure element intensities [34] are used. If the intensity $I_{i, E}^{Z}$ emitted from the pure element having the exact thickness of the thin film specimen could be obtained, assuming both unknown 
and standard to be measured under identical experimental conditions, 1.e., same substrate, specimen tilt, incident beam energy and current, then the ratio

$$
I_{1, E}^{+} / I_{1, E}^{z}=C_{i}^{+}
$$

where $C_{i}$ is the true concentration of element " $i$ " in a film of thickness z. Equation (16) is valid since absorption, electron scattering, and retardation as well as fluorescence effects are negligible in thin films. The value of $\mathrm{I}_{i, E}^{z}$ can be calculated as a function of $z$ as has been described. The value of $I_{i, E}^{+} / I_{i, E}$ can be measured. But the film thickness $z$ is unknown.

However, if the ratio $I^{+} / I_{E}$ can be obtained for all components of the thin film specimen, a good approximation to $z$ can be made. This possibility exists because emitted $\mathrm{X}$-ray intensity from a thin film is a nearly linear function of thickness as shown by experimental results [21], [22]. So we presume $I^{+} / I_{E}$ data for all components to be measured; we can calculate $I_{E}^{z} / I_{E}$ for all components straightforwardly. We may then obtain the value of $z$ for which $I_{E}^{z} / I_{E}$ is equal to $I^{+} / I_{E}$; calling this value $(z)_{i}$ for the " $i$ th" element, we may similarly obtain $(z)_{j}$ and so on to $(z)_{n}$ for an " $n$ " component sample. Finally,

$$
z=(z)_{i}+(z)_{j}+\cdots+(z)_{n}
$$

where $z$ is the mass thickness of the unknown thin film specimen. Thus the intensities from a homogeneous mixture of thickness $z$ are equivalent to the intensities from a hypothetical layered structure of thickness $(z)_{i}+(z)_{j}+\ldots+(z)_{n}=z$. 
Having thus obtained the value of $z$, we may now obtain $I_{1, E}^{z} / I_{i, E}$. Then

$$
\frac{I_{1, E}^{+}}{I_{i, E}} \div \frac{I_{i, E}^{2}}{I_{i, E}}=\frac{I_{i, E}^{+}}{I_{i, E}^{2}}=C_{i}^{+}
$$

These assertions can be tested using the experimental data of Kyser and Murata for $\mathrm{Mn}-\mathrm{Bi}$ and $\mathrm{Co}-\mathrm{Pt}$ films on $\mathrm{Al}_{2} \mathrm{O}_{3}$ substrates [22]. Figure (8) shows $I_{E}^{z} / I_{E}$ for all of these elements as a function of $z$. Table IV lists the measured values of $\mathrm{I}_{i, E}^{+} / I_{i, E}$ reported for several specimens. Table IV also gives the $z$ values found from using Fig. ( 8 ) and Eqn (17) and the $C_{i}$ values subsequently obtained by using Eqn (18) as compared with those reported by Kyser and Murata [22].

\section{Extension to Spherical and Cylindrical Particles}

The major difference between the particle and thin film case is that the number of electrons which escape the particle of interest depends on the particle size; Fig. (9) shows the effect of bombarding spheres of Al with electron beams of varying incident energy. Calling the fraction of electrons escaping from the particle $n_{L}$, experimental evidence as well as Monte Carlo calculations led to the formulation of an empirical expression for $n_{L}$ :

$$
\eta_{L}=1-d p[(1-n) / R]
$$

where $d_{p}$ is the particle diameter and $n$ and $R$ are as defined previously. If $\eta_{L}$ is substituted into Eqns (3) and (5a), then values of $\phi_{0}, h$ and $k$ for particles can be obtained. Then the ratio of generated intensities from the particle to the bulk can be obtained for the particle in an 
analogous fashion to that discussed previously (Eqns 12,13 ). However, this ratio is not sufficient by itself since the particle shape must be taken into account as well. By using Monte Carlo calculations for comparison, the best approximation for spheres and cylinders appears to be:

$$
k_{i}^{p}=\left(\frac{I_{G}^{P}}{I_{G}}\right)^{3}\left\{1+\left[\frac{d}{z_{r}}(f(x)-1)\right]\right\}\left(\frac{100}{100+3 Z}\right)
$$

where $I_{G}^{P}$ is the generated intensity for the particle, $d_{p}$ is the particle diameter and all other terms are as defined previously. Equation (20) is only valid up to $d_{p}=z_{r}$. Then $k_{r}^{p}$ becomes unity. However, some of the generated intensity will be lost due to the shape of the particle as shown schematically in Fig. (10). For spheres and cylinders the volume ratio of the segment containing the $\mathrm{X}$-ray emission with respect to that of the bulk is given by

$$
\frac{v_{p}}{v_{z_{r}}}=\frac{1.5 d_{p}-z_{r}}{2 z_{r}}
$$

when $d_{p}>z_{r}$. Equation (21) predict.s that this ratio is unity when $d_{p}=2 z_{r}$. Hence, for the region $z_{x}=d_{p} \leq 2 z_{x}$ we have

$$
\mathrm{k}_{i}^{\mathrm{p}}=\mathrm{v}_{\mathrm{p}} / \mathrm{v}_{z_{\mathrm{r}}}
$$

The need for the cubing in Eqn (20) is apparently due to the 3 dimensional $\mathrm{X}$-ray distribution in depth; we are investigating this distribution in more detail. Figures (11)\&(12) show results from Eqns (20) and (22) as compared with Monte Cerlo calculations for particles. Agreement is reasonably good. 
The LaPlace transform of the function $\phi(z)$ is given by:

$$
F(0)=\int_{0}^{\infty} \phi(z) d z
$$

If there were no absorption of electron beam produced $\mathrm{X}$-rays by the sample, then the intensity of emitted $X$-rays would be $F(0)$. But as these X-rays travel out of the specimen in a direction toward the detector, they suffer absorption according to Beer's Law viz, $\phi(z) e^{-\chi z}$ where $\chi$ is $(\mu / \rho) \csc \psi, \psi$ being the average angle of emergence of the $X$-rays from the specimen and $(\mu / \rho)$ being the mass attenuation coefficient for the $\mathrm{X}$-ray line of interest. In this situation,

$$
F(x)=\int_{0}^{\infty} e^{-\chi z} \phi(z) d z
$$

The intensity of emitted $\mathrm{X}$-rays is given by:

$$
I_{E}=I_{G} \frac{F(x)}{F(0)}=I_{G} f(x)
$$

This absorption correction factor, $f(x)$ has been the subject of much study [ 8$],[14],[23],[27]$. The value of $f(x)$, which ranges from 0 to 1 , is probably the single most important parameter in quantitative X-ray microanalysis. Accurate $f(X)$ values are especially crucial for soft $X$-ray analysis, i.e., when $x$ is large. In fact, the accuracy of $X$-ray microanalysis deteriorates rapidly if $f(X)<0.7$ [23], [35]. One reason that $f(X)$ is not well known is that few determinations of this parameter have been made experimentally and also that few $\phi(z)$ curves have been available. With the relation given in Eqn (2), one can derive an expression for $f(x)$ : 
$f(x)=\left\{x^{-3}\left[0.25 x^{2}\left[4 \phi_{0}-e^{-1.5 h x}\left(\phi_{0}+3 k\right)\right]+x\left[h^{-1}\left(k-\phi_{0}\right)\left(e^{-1.5 h x}+2\right)+\right.\right.\right.$

$$
\begin{aligned}
& \left.2 h^{-2}\left(\phi_{0}-k\right)\left(1-e^{-1.5 h x}\right)\right]- \\
& \left.\left[\frac{0.25\left(\phi_{0}+3 k\right) e^{-1.5 x h}\left(z_{r}-1.5 h\right)}{1.5 h x-x^{2} r^{-2}}\right]\right\} \frac{8}{\left(\phi_{0}+3 k\right)\left(z_{r}+1.5 h\right)}
\end{aligned}
$$

This rather cumbersome expression -- compare with Eqn (1) -- is easily programmed for computer reduction. Its chief value should lie in the region $0.5 \geq f(x)$. As an example, the value of $f(x)$ from Eqn (26) is plotted against $x$ for $\mathrm{Cu}-\mathrm{L} \alpha$ \& $\mathrm{E}_{\mathrm{o}}=25 \mathrm{keV}$ in Fig. (13). The results compare well with the Monte Carlo derived curve.

The Atomic Number Effect Correction in X-ray Microanalysis

The so-called atomic number correction arises because the specimen is usually different in average atomic number than the standard. This difference is reflected in differences in electron backscatter and retardation between sample and standard. This correction is often called $k_{z}[8]$. In fact,

$$
k_{z}=\frac{F(0) \text { STD }}{F(0) \text { SAMPLE }}
$$

But since $F(0)=0.125\left(\phi_{0}+3 k\right)\left(z_{r}+1.5 h\right)$ based on Eqn (2), then

$$
k_{z}=\frac{Y_{1}-Y_{2} W}{Y_{1}^{*}-Y_{2}^{*} W}
$$

where $\mathrm{Y}_{1}=(1+2.8 n)\left(1+1.5 \mathrm{~h}_{0}\right), \mathrm{h}_{0}$ being $\mathrm{h} / \mathrm{z}_{\mathrm{r}}$

$$
Y_{2}=n\left(2.52+3.78 h_{0}\right)
$$




$$
\begin{aligned}
& Y_{1}^{*}, Y_{2}^{*}=Y_{1} \text { and } Y_{2} \text { for the sample } \\
& h_{0}=10^{-5} Z^{*}+z^{-1 / 2} \\
& \eta^{*}=\sum C_{i} \eta_{i} \\
& Z^{*}=C_{i} Z_{i}, C_{i} \text { being the true mass fraction of the } i \text { th element }
\end{aligned}
$$

in the specimen. In practice, $Y_{1}^{*}$ and $Y_{2}^{*}$ must be obtained from the iteration loop of the correction program. Equation (28) has been tested for $\mathrm{Ag}-\mathrm{Au}$ and $\mathrm{Au}-\mathrm{Cu}$ alloys (SRM 481, 482) comprising four compositions and a range of incident beam energies. The results are good as shown by the comparison in Tables $\mathrm{V}$ and $\mathrm{VI}$.

\section{Energy Dispersive X-ray Spectrometry}

An energy dispersive $\mathrm{X}$-ray spectrometer (EDS) was first used for electron microanalysis in 1968 [36]. Since then the number and quality of EDS systems has steadily and rapidly increased. Today a large majority of scanning electron microscopes (SEM) and many transmission electron microscopes (TEM) are equipped with EDS facilities. Thus, the electron microscopist can perform qualitative and quantitative analyses and can also map elemental distribution in the area scanned by the electron beam.

\section{EDS Systems}

The heart of the EDS detector is a $p-i-n$ (p-type, intrinsic, n-type) lithium-drifted silicon crystal. X-rays generated from the specimen are absorbed in the intrinsic region creating electron-hole pairs. These pairs are separated and collected by bias applied to the Si(Li) crystal to form a charge pulse. A charge sensitive field-effect- 
transistor preamplifier converts this pulse to a voltage pulse. After further amplification and pulse shaping, this signal is sent to a multichannel analyzer (MCA). The function of the MCA is to collect, classify, and store each pulse. Pulse voltage remains proportional to Initial photon energy. Pulse amplitude is converted to time so that an input signal can be linearly converted to some number say between 0 and 1024. This number is proportional to the incident $\mathrm{X}$-ray photon energy from the sample and also serves as an address of storage location in the MCA memory. Thus, if a 5 volt pulse corresponded to address (channel) 1024, a 1 volt pulse arriving at the MCA would produce an addition of one unit at channel 205. The energy distribution spectrum is built up in this way over a period of time; the spectrum can be displayed or sent to a computer for further processing.

Detector resolution is typically quoted at 150 to $170 \mathrm{eV}$ at a $5.9 \mathrm{keV}$ reference energy [37]. The resolution figure usually meant is the full width at half-maximum (FWHM) of the peak. There are two contributions to the value, (1) the inherent statistical aspect of the energy transfer from the photon in the Si(Li) crystal and (2) electronic noise arising from the preamplifier. If the electronic noise were reduced to zero, then the theoretical resolution (FWHM) at $5.9 \mathrm{keV}$ would be about $100 \mathrm{eV}$ [37]. In practice, noise reduction is accomplished by cooling the $\mathrm{Si}(\mathrm{Li})$ crystal as well as the first-stage preamplifier field effect transistor to about $77^{\circ} \mathrm{K}$. (This cooling also prevents the lithium from diffusing out of the intrinsic region.) Noise reduction can also be obtained by using pulsed optical feedback systems. These systems activate a light-emitting diode when the preamplifier output reaches a 
preset voltage level. The diode causes a leakage current to flow in the field-effect-transistor which restores it to the initial operating point [38]. This scheme eliminates noise usually associated with resistive feedback in conventional preamplifiers.

As stated, present-day EDS systems are supplied with resolution figures quoted at about $150 \mathrm{eV}$; in 1968, the figure was $600 \mathrm{eV}$. Most of the reduction was due to improved noise minimization procedures. However, this means of resolution improvement is nearly exhausted [39]. Thus, in the near future, dramatic resolution improvement is unlikely. Complete details concerning the principles and operation of energy dispersive $\mathrm{X}$-ray spectrometer systems can be found in the literature [37]-[41].

\section{Interpretation of Spectra}

The MCA is usually calibrated to correspond to some convenient energy increment, say 20 or $50 \mathrm{eV}$, per channel. For example, a 1024 channel $\mathrm{MCA}$ with a $20 \mathrm{eV}$ per channel calibration will collect all peaks in the energy range 0 to $20.5 \mathrm{keV}$. Moseley's Law relates the characteristic energy of an X-ray peak and the atomic number, $Z$, of the element responsible for the peak. In the context of an EDS spectrum, Moseley's Law can be expressed as:

$$
z=\exp \left[K_{1}\left(\operatorname{lnc}_{n}+K_{2}\right)\right]
$$

where $C_{n}$ is the peak channel number and $K_{1}$ and $K_{2}$ are numerical coefficients depending on the MCA calibration. Thus, appropriate atomic numbers can be assigned for the elements present in the electron irradiated region. Collection of the spectrum often takes only one or tio minutes; hence, qualitative analysis is in principle very rapid and spectral.interpretation is simple. 
However, there are some major potential sources of difficulty in using EDS techniques. These include (1) pulse pile-up effects; (2) difficulties in accurate determination of the spectral background caused by the X-ray continuum; (3) overlaps or interferences occurring when spectral peaks are closer in energy than the resolution of the EDS system; (4) problems associated with positive location and identification of weak peaks in the spectrum, i.e., detectability limits; and (5) difficulties associated with analysis of elements with $4 \leq z \leq 11$, i.e., the light elements

\section{(a) Pulse Pile-up}

The main amplifier of the EDS system can only process incoming signal pulses at some finite rate. When the time interval between pulses approaches this processing time, pulse pile-up results. Thus, high input count rates to the EDS system can cause pulse pile-up which appears as an energy distortion of one or both of the two closely spaced pulses. The net effect is to cause such pulses to be added to channels representing higher energy than the true pulse energy. Thus, extraneous peaks may appear in the final spectrum causing confusion and potential error in its interpretation. Particularly insidious is the appearance of sum-peaks representing the effective coincidence in time and summation of two strong monoenergetic pulses, e.g., $K \alpha+K \alpha, K \alpha+K \beta, K \beta+K \beta$, within the timing resolution of the detection and pulse processing system [37], [39].

Therefore, most modern EDS systems provide for pulse pile-up rejection by inserting a circuit to inspect the rate at which pulses arrive in the main amplifier. This circuit will switch the ICA input 
off should a second pulse arrive before the main amplifier processes Its predecessor. Pulse shaping time in the amplifier interacts as well; shorter shaping times permit higher input count rates at the expense of energy resolution. At present, input rates of 15 to 20,000 counts per second can be achieved without output count rate loss or serious resolution degradation in conventional systems [37], [39]. Special systems, such as switched beam apparatus, appear capable of accepting even higher input count rates [42].

All of the pulse processing methods cause the MCA to be inactive for finite time periods after arrival of a pulse. Hence, most MCA's have a so-called live-time option. If this option is selected, then counting time is adjusted to compensate for these inactive periods. Any comparative measurements ought to be performed with the live-time option [39].

(b) Background Correction

The development given here is based on a method developed by Fiori et al. [43] following Ware and Reed [44] and Lifshin et al. [39]. For background correction with an EDS system, an estimate of the continuum background intensity below a peak having photon energy, E, is required. Therefore, absorption of generated continuum photons within the specimen must be taken into account. Furthermore, the efficiency for photon absorption in the detector window and p-type silicon dead layer in the $\mathrm{Si}(\mathrm{Li})$ crystal as well as for transmission of photons through the Si(Li) crystal must be considered. Thus, the number of detected continuum photons $\mathrm{N}_{E}$ within the small energy range $E$ to $E+\Delta E$ may be written as: 


$$
\mathbb{N}_{E}=I \Delta E f_{E} P_{E}
$$

where $I \Delta E$ is the probability of photon production (due to one electron) in the energy range from $E$ to $E+\Delta E$. The form of the absorption effect designated $f_{E}$ has been expressed as [43]

$$
\begin{aligned}
1 / f_{E} & =\left(1+1.2 \times 10^{-6} \gamma X\right)^{2} \\
\gamma & =E_{0}^{1.65}-E^{1.65}
\end{aligned}
$$

$E_{0}$ being the incident electron beam energy and

$\chi=\csc \psi[M($ target, $E)]$ with

$\psi$ being the $X$-ray emergence angle and

$M($ target, E) being the X-ray mass attenuation coefficient of the target for photons of energy $E$.

The value of $M($ target, $\dot{E})$ is $\Sigma C_{i} M_{i}$ where $i$ refers to the $i^{\text {th }}$ element in the target. Hence, the background correction is a function of the target composition, since $C_{i}$ is the true mass fraction of element $i$. The detector efficiency, $P_{E}$, can be expressed as

$$
P_{E}=\exp -\left\{M(B e, E) t_{B e}+M\left(S i^{D}, E\right) t_{S i}^{D}\right\}\left\{1-\exp -\left[M(S i, E) t_{S i}\right]\right\}
$$

where $M(B e, E), M\left(S_{i}^{D}, E\right), M(S i, E), t_{B e}, t_{S i}^{D}$ and $t_{S i}$ are the respective $\mathrm{X}$-ray mass attenuation coefficients and mass thicknesses of the Be window, Si dead zone and Si active zone. There is also a gold conductive layer about $20 \mathrm{~nm}$ thick flashed onto the Si(Li) crystal which does not contribute significantly to $P_{E}[43],[44]$.

Lifshin et al. found that ILE in Eqn. (30) could be expressed as [39]: 


$$
I \Delta E=\bar{z}\left[a\left(E_{0}-E\right) / E+b\left(E_{0}-E\right)^{2} / E\right]
$$

where $\bar{Z}$ is the average atomic number of the target equivalent to $\sum C_{i} Z_{i}$ and "a" and "b" are empirical fitting factors.

In practice, $\mathrm{N}_{\mathrm{E}}$ is measured at two widely separated points in the spectrum, both free of all peak interferences and "a" and "b" determined by solving the resulting two equations in two unknowns. Then the entire curve of $N_{E}$ as a function of energy can be calculated. Figure (14) shows an as-collected spectrum of the mineral Kakanui Hornblende while Fig. (15) shows the background corrected spectrum. This method of background correction has been tested by comparison with measured spectra and by application in quantitative X-ray analyses [43].

(c) Peak Interference

Many X-ray peaks lie within $100 \mathrm{eV}$ of one another, e.g., $\mathrm{Pb}-\mathrm{M}$ and S-K [45]. Hence, peak overlap or interference may occur in the EDS spectrum. The usual means of attacking this problem is by means of spectrum stripping. However, to carry out spectrum stripping properly usually requires computer processing of the spectrum.

Gaussian parameters of each peak detected in the spectrum may be written as follows:

$$
I_{S}=I_{p} \exp -\left[\left(E-E_{p}\right)^{2} /\left(0.6 R_{H}\right)^{2}\right]
$$

where $I_{S}$ is the stripped intensity at energy $E$

$I_{p}$ is the peak intensity

$E_{p}$ is the peak energy and

$R_{H}$ is the detector resolution (FWHM) at energy $E_{P}$. 
For Eqn (34) to be valid, the background due to the continuum must be accurately subtracted from the raw spectrum.

In practice, spectrum stripping is carried out by stripping major peaks from the spectrum in regions where overlap is suspected or known to be present. Consider the spectrum in Fig. (15) in the region around the CaK $\alpha$ peak. The $\mathrm{K}-\mathrm{K} \alpha$ peak lies on the low energy shoulder of the CaK $\alpha$ peak; the $K-K \beta$ peak is totally obscured. For quantitative analysis of calcium and for proof positive of the existence of the $K-K \beta$ peak in the spectrum, one would have to first estimate the $K \alpha / K \beta$ ratio in potassium in the spectrum and then strip the $K B$ potassium peak from the calcium Ka peak. Results are shown in Fig. (16).

At this point, the need for computer interfacing with the EDS system should be clear. Background correction and spectrum stripping both require such a facility. The most advantageous arrangement would be for real-time interaction of the operator, EDS system and computer. For example, background correction could be carried out and the result. displayed on the MCA. Spectrum stripping could then be performed interactively by the operator in control of the computer. The stripped spectrum would be displayed for immediate analysis [46]. Furthermore, computer data reduction is required for quantitative analysis; sma11 on-line programs exist for this purpose [47].

(d) Detection of Weak Peaks

Lifshin and co-workers [39], [48] have pointed out that the limiting count-rate in an EDS system, which was suggested to be $15-20,000$ counts per second, is the total system count-rate for all energies. This fact can lead to serious difficulties if one is attempting to optimize 
sensitivity for a weak peak since the accompanying stronger peaks from other elements contained in the specimen will account for most of the observed total count-rate [48]. In addition, even with pure elements, peak-to-background ratios with the EDS system are usually less than 50 to. 1 and often less than 30 to 1 [49]. Thus, unambiguous detection of weak peaks in the spectrum may be difficult.

Such considerations underscore the need for accurate background corrections and adequate spectrum stripping methods via the interactive computer mode. Nevertheless, after these procedures have been carried out, there is perhaps no better instrument than the human eye-brain combination for deciding whether some feature in the spectrum is worth further investigation. Then a long collection time can be used to ascertain whether a real peak is present. Clearly, this procedure is subject to compromises dictated by available time, potential specimen damage by the primary beam and instrument stability.

The detectability limit of EDS facilities has been compared to that attainable with wavelength dispersive spectrometers [39], [49]. For example, elemental concentrations of silicon or molybdenum on the order of $0.1 \%$ were easily detected in steel standards [49] by EDS. Usually, EDS and wavelength dispersive methods give comparable detection limits [48].

(e) Light Element Analysis

Because of $\mathrm{X}$-ray absorption in the beryllium window of the detector (typically 8-12 $\mu \mathrm{m} \mathrm{Be}$ is used) and in the silicon dead-layer, elements with atomic number less than eleven are usually not analyzed by means of EDS methods. For example, the detector efficiency, $\mathrm{P}_{E}$, at the energy 
of oxygen $\mathrm{K} \alpha(0.52 \mathrm{keV})$ is about $0.2 \%$ if an $8 \mathrm{~m} \mathrm{Be}$ window intervenes [37]. But if the window is removed and Eqn (32) solved for $P_{E}$ with $\mathrm{t}_{\mathrm{S} 1}^{\mathrm{D}}$ of $0.2 \mu \mathrm{m}$ and a mass attenuation coefficient of $\mathrm{Si}$ for oxygen $\mathrm{K} \alpha$ of 8790 [50], then $P_{E}$ is $66 \%$. Therefore, windowless detectors offer a means for analysis of the light elements. However, the characteristic energies of adjacent elements in the region $11>\mathrm{Z}$ are $150 \mathrm{eV}$ or less. Hence, adjacent peaks will probably not be clearly resolved with EDS facilities.

Windowless detector systems have been developed [51], [52] and are available. With such systems, elements down to carbon have been observed and a detectability limit of about $0.4 \%$ for oxygen in aluminum found [51]. Oxide thicknesses of $\mathrm{SiO}_{2}$ on silicon substrates have been successfully measured [52]. Flow proportional counters having an ultrathin window can be used for the detection of light elements as well.

\section{Quantitative Analysis}

All schemes for carrying out quantitative analysis use a standard of known composition. In many cases, especially metals, pure elements are suitable. In the case of mineral or petrological samples, homogenous, compound standards close in average atomic number to the unknown are usually chosen. What the analyst measures is the relative $\mathrm{X}$-ray intensity ratio from the elements of interest in the specimen to the same elements in the standard. Both specimen and standard are examined under identical experimental conditions. The measured relative intensity ratio (commonly called $k$ ) must be accurately determined or any quantitative analysis scheme will result in the same inaccuracy. Peak intensities 
can usually be measured more easily and with better accuracy than integrated intensities. Using peak intensity values gives results as accurate as Integrated intensities [53]; hence, in all that follows, peak intensity is meant.

A useful exercise is to insert a pure element and treat one side as "specimen" and the other as "standard." Then have several operators measure "relative intensity" values (one side to the other) using different operating conditions. The results will indicate the maximum accuracy with which a relative intensity ratio can be obtained; values of 1.00 for the ratio in this test will not be obtained except by good fortune.

Once the $k$ values have been obtained, they must be corrected for several effects including (1) effects resulting from the nature of the differences in electron scattering and retardation between the specimen and the standard, i.e., the so-called atomic number effect, $k_{Z}$, absorption of $\mathrm{X}$-rays within the specimen, $\mathrm{k}_{\mathrm{A}}$, (3) fluorescence effects, $k_{F}$, and (4) continuum fluorescence, $k_{c}$. A common form of the correction equation is

$$
\mathrm{C}=\mathrm{k} \cdot \mathrm{k}_{\mathrm{Z}} \cdot \mathrm{k}_{\mathrm{A}} \cdot \mathrm{k}_{\mathrm{F}} \cdot \mathrm{k}_{\mathrm{C}}
$$

where $\mathrm{C}$ is the weight fraction of the element of interest. This method is often referred to as the $\mathrm{ZAF}$ method. Each of the effects 1isted has been studied in detail [54] and reviews have been written [ 8 ], [55], [56]. Empirical methods for data reduction have also been developed [57], [58]. A procedure using a mini-computer for on-line correction of $\mathrm{X}$-ray data from electron microprobe analysis has been developed [47]. This 
program, called FRAME, allows mass fractions to be computed on-line by the ZAF approach. Besides X-ray intensities, the only input data required are (1) the atomic numbers of the elements present, (2) the analytical Iine being used, and (3) the operating voltage. Other required parameters such as atomic weights and $\mathrm{X}$-ray mass attenuation coefficients are stored or calculated by the program. FRAME need not be used on-line. If desired, the program can be run as a batch or time-shared program for all analyses. The program is flexible, short, simple to use, and accurate. Furthermore, the absorption term $f_{E}$ in the expression for background subtraction in EDS spectra (Eqns 2,3) depends upon the specimen concentration. Such a program is needed then in order to obtain $f_{E}$ properly and in turn to carry out the background correction. Clearly, incorrect background subtraction will lead to errors in quantitation.

The correction procedure FRAME $B$ has been developed for electron probe microanalysis with a lithium-drifted silicon detector. This program, which computes on-line mass fractions of the elements contained in the specimen, is a modification of the correction procedure FRAME [33]. The input data required are: X-ray intensities from the peaks of interest and the continuum intensities at two energies, the atomic numbers of all the elements present, the analytical lines used and the operating voltage. Other required parameters, such as atomic weights, the X-ray emergence angle and X-ray mass attenuation coefficients, are stored or calculated by the program. FRAME B is in FORTRAN IV; it requires less than 5,000 words of conputer core, and will handle up to 13 elements The continuous spectrum for a specific set of conditions is obtained as follows. From the value of $N(E)$ measured at two energy levels where 
there are no characteristic lines or detector artifacts (incomplete charge collection, pulse pile-up, double energy peaks and escape peaks), the parameters $a$ and $b$ in Eqn (33) are calculated. They in turn define, through Eqn (30), the value of $N(E)$ at an energy $E$.

As pointed out by Ware and Reed [44], the absorption term $f_{E}$ is dependent upon the composition of the target. Therefore, when the composition of the target is unknown, it is necessary to include Eqn (31) in an iteration loop as shown in Fig. (17). This flow diagram is a simplified description of the program.

\section{Elemental Mapping and $\mathrm{SiC}$ in Al Composite}

The technique of $\mathrm{X}$-ray area scanning provides the investigator with what amounts to a scanning X-ray microscope. The amplified signal from the detector system -- energy-dispersive or crystal spectrometer -is made to modulate the brightness of a cathode ray tube (CRT) scanned in synchronism with the electron probe. Thus, on the CRT, a picture is obtained using the spatial varıacıon of X-ray emission from the surface. The same magnification controls, sweep system and amplifier as in the scanning electron microscope, are used. An X-ray area scan can show tones ranging from black to white, depending on experimental conditions. In places of high concentration of the element in the scanned area, the picture will be nearly photographically white; it will be gray where the element's concentration is lower and black where the element is absent.

When an energy-dispersive system is used to prepare the area scan photograph, care must be taken to ensure that no other peak interferes with the signal of the desired element. The desired peak should be 
carefully isolated by means of a single-channel analyzer; this analyzer Is often built into multichannel analyzers. The amplified output of the single-channel analyzer is displayed on a CRT and so provides the elemental distribution desired. Magnification can be varied in the usual way. Separate micrographs of all elements of interest in the scanned area can be built up to give the complete elemental distribution. A detailed discussion of $\mathrm{X}$-ray area scanning has been given by Heinrich [59].

In the case of an energy-dispersive detector, the time required to build up a satisfactory $X$-ray area scan may be very long. This hindrance results from the requirement imposed by Poisson statistics to provide at least 20,000 photons per micrograph; frequently 200,000 are needed and occasionally as many as 500,000 photons must be used. For the SEM, often run with beam currents on the order of $10^{-10}$ or $10^{-11} \mathrm{~A}$, the total number of photons produced is fairly low ( $10^{4}$ per second). Since one peak obtained from the total spectrum is of interest, the time to obtain a satisfactory micrograph is correspondingly long. Times in excess of 15 min are not uncommon in the preparation of $\mathrm{X}$-ray area scans.

If the specimen will not be harmed, higher beam currents are useful -- not only for elemental mapping but for all phases of X-ray analysis. The loss of resolution resulting from increasing beam current matters little since the X-ray emission volume is largely determined by electron diffusion and not by probe size except for thin specimens. Since it is difficult to decrease the effective volume of $\mathrm{X}$-ray emission below $1 \mu \mathrm{m}, \mathrm{X}$-ray scanning pictures are limited to magnifications of about 3000 diameters. 
Some further studies using elemental mapping were made on the SiC In aluminum composite provided by Marshall Space Flight Center. The manufacturing method has led to the formation of several phases: 1) SiC unreacted, 2) Al-Si, 3) Al-Si-C, 4) Al-C and silicon is present in the grain boundaries. The tungsten phases produced by aluminum reaction with the tungsten core of the SiC filament are $\mathrm{Al}_{4} \mathrm{~W}$ and $\mathrm{AI}_{3} \mathrm{~W}$. Less than $25 \%$ of the diameter of a SiC filament remains as SiC; the remainder has reacted. The results are shown in Fig. (18).

Discussion and Conclusions

A simple analytical method for the analysis of thin film laminate composite specimens has been devised. This method can be used to calculate film thickness and/or film composition. If a substrate is present, its composition is presumed known. For compositional deternination, the method suffers the drawback that all elements present must have their intensities measured. Results from this method agree with results reported in the literature to within about $\pm 10 \%$ relative.

For pure element films, the thin-film technique predicts the intensity ratio of film-to-bulk for thicknesses up to the $\mathrm{X}$-ray range, i.e., effectively bulk material. In the analysis of multielement films, the thickness range over which the technique can be applied is more limited. This is a result of the assumption that a thin, multicomponent film can be approximated as a layered structure of the pure element but having the same total mass thickness. As long as there is negiigible elastic scattering within the film, this approximation appears to be reasonable. As the thickness is increased, elastic scattering, which is composition 
dependent, will cause this simple approximation to fail. The available data for binary films permitted us to test the thin film technique up to a mass thickness of $100 \mu \mathrm{g} / \mathrm{cm}^{2}$ in Co-Pt and $\mathrm{Mn}-\mathrm{Bi}$ samples in which the Intensity from the thin film is about $20 \%$ of the bulk value. Further testing with data from thicker films is necessary to determine the range of thickness where the technique is applicable. The method can be used with tilted specimens in principle. However, no experimental data comparisons have as yet been carried out for these cases. The computational procedure is not overly cumbersome; results can be obtained with a desk calculator in a few minutes. In addition, the distribution in-depth of electron beam produced X-rays in a solid can be deduced with this method; agreement with published data is good.

The method can be extended to composites consisting of particulate reenforcers in a metal matrix provided the geometry of the particles is specified or known. The extension has been carried out for spherical and cylindrical particles. 
1. T. A. Hall in Quantitative Electron Probe Analysis (K. F. J. Heinrich, ed.) NBS Spec. Publ. 298, 269 (1968).

2. T. A. Hall in Physical Techniques in Biological Research (G. Oster, ed.) Vol. IA, P. 157 (1971).

3. T. A. Hall and P. D. Peters in Microprobe Analysis as Applied to Cells and Tissues (T. Hall, P. Echlin, and R. Kaufmann, eds.) Academic (New York and London) P. 229 (1974).

4. R. Tixier and J. Philibert, J. de Microscopie 1, 588 (1968).

5. M. H. Jacobs and J. Baborovska, Proc. 5th Eur. Conf. Elect. Micros. (Inst. Phys. London) p. 136 (1972).

6. G. Cliff and G. W. Lorimer, Proc. 5th Eur. Conf. Elect. Micros. (Inst. Phys. London) p. 140 (1972).

7. J. I. Goldstein and J. W. Colby in Practical Scanning Electron Microscopy (J. I. Goldstein and H. Yakowitz, eds.) Plenum (New York), p. 470 (1975).

8. H. Yakowitz in Practical Scanning Electron Microscopy (J. I. Goldstein and H. Yakowitz, eds.) Plenum (New York), p. 327 (1975):

9. J. W. Colby, Adv. X-ray Anal. 11, 287 (1968).

10. Y. Oda and K. Nakajima, J. Jap. Inst. Met. 37, 673 (1973).

11. R. Castaing, Thesis to Univ. of Paris (1951).

12. R. Castaing and J. Henoc, Proc. 4th Int'I Congr. X-ray Optics and Microanalysis (R. Castaing, P. Deschamps and J. Philibert, eds.) Hermann (Paris) p. 120 (1966).

13. P. Duncumb in Electron Microscopy and Analysis, Conf. Ser. 非0 (Inst. Phys. Lond), p. 132 (1971).

14. R. Castaing and J. Descamps, J. Phys. Radium 16, 304 (1955).

15. J. D. Brown and L. Parobek, Proc. 6th Int'l Congr. X-ray Optics and Microanalysis (G. Shinoda, K. Kohra and T. Ichinokawa, eds.) Univ. Tokyo Press, p. 163 (1972).

16. J. W. Criss and L. S. Birks in The Electron Microprobe, (T. D. McKinley, K. F. J. Heinrich, and D. B. Wittry, eds.) Wiley (New York) p. 217 (1966).

17. W. Reuter, Proc. 6th Int'1. Congr. X-ray Optics and Microanalysis (G. Shinoda, K. Kohra, and T. Ichinokawa, eds.) Univ. Tokyo Press, p. 121 (1972). 
18. G. Shinoda, K. Murata, and R. Shimizu in Quantitative Electron Probe Microanalysis (K. F. J. Heinrich, ed.) NBS Spec. Publ. 298, 155 (1968).

19. Proceedings of Workshop on Monte Carlo Calculations in Electron Probe Microanalysis (K. F. J. Heinrich, H. Yakowitz, and D. E. Newbury, eds.) NBS Spec. Pub1. (1976) - in press.

20. H. E. Bishop and D. M. Poole, J. Phys. D: App1. Phys. $\underline{6}, 1142$ (1973).

21. R. B. Bolon and E. Lifshin, SEM/73 (0. Johari, ed.) IITRI (Chicago), p. 285 (1973).

22. D. F. Kyser and K. Murata, IBM J. Res. Devel. 18 (4), 352 (1974).

23. K. F. J. Heinrich and H. Yakowitz, Analytical Chemistry -- in press.

24. K. F. J. Heinrich, Proc. 4th Int'1. Congr. X-ray Optics and Microanalysis (R. Castaing, P. Deschamps and J. Philibert, eds.) Herman (Paris), p. 159 (1966).

25. H. E. Bishop, Proc. 4th Int'1 Congr. X-ray Optics and Microanalysis (R. Castaing, P. Deschamps and J. Philibert, eds.) Hermann (Paris) p. 153 (1966).

26. D. J. Fathers, J. P. Jakubovics, D. C. Joy, D. E. Newbury and H. Yakowitz, phys. stat. sol. (a) 22, 609 (1974).

27. C. A. Andersen and M. F. Hasler, Proc. 4th Int'1. Congr. X-ray Optics and Microanalysis (R. Castaing, P. Deschamps, and J. Philibert, eds.) Hermann (Paris), p. 310 (1966).

28. S. J. B. Reed, Proc. 4th Int'1. Congr. X-ray Optics and Microanalysis (R. Castaing, P. Deschamps, and J. Philibert, eds.) Hermann (Paris), p. 339 (1966).

29. P. Duncumb, Proc. 2nd Int'1. Congr. X-ray Optics and Microanalysis (A. Engström, V. E. Cosslett, and H. Pattee, eds.) Elsevier (Amsterdam), p. 365 (1960).

30. K. F. J. Heinrich, private communication (1975).

31. M. Green, Thesis to Cambridge University (1962).

32. K. Kanaya and S. Okayama, J. Phys. D: App1. Phys. $\underline{5}, 43$ (1972).

33. C. Fiori, R. L. Myklebust, K. F. J. Heinrich and H. Yakowitz, Proc. Microbeam Analysis Soc. (10th Nat'1. Conf.) Las Vegas, Nev. (1975) Paper $\|^{20}$.

34. J. C. Russ, Proc. Microbeam Analysis Soc. (9th Nat'1. Conf.) Ottawa, Can. (1974) Paper \#22. 
54. Quantitative Electron Probe Microanalysis, (K. F. J. Heinrich, ed.) NBS Spec. Pub1. 298 (1968).

55. S. J. B. Reed in Microprobe Analysis (C. A. Andersen, ed.) Wiley, New York (1973) p. 53.

56. S. J. B. Reed, Electron Microprobe Analysis, University Fress, Cambridge $(1975)$.

57. T. O. Ziebold and R. E. Ogilvie, Anal. Chem. 36,322 (1964).

58. A. E. Bence and A. Albee, J. Geo1. 76, 382 (1968).

59. K. F. J. Heinrich, NBS Tech. Note 278 (1967).

60. M. Bayard in Microprobe Analysis (C. A. Andersen, ed.) Wiley, New York (1973) p. 323. 
Table I: Comparison of Thin Film Data of Reuter [17] with NBS Results

\begin{tabular}{|c|c|c|c|c|c|c|c|}
\hline Film & Substrate & $E_{0}$ & $\begin{array}{c}z \\
\left(\mu \mathrm{g} / \mathrm{cm}^{2}\right)\end{array}$ & $k_{R}$ & $k_{\text {NBS }}$ & ${ }^{z}$ NBS & ${ }^{2}$ REUTER \\
\hline $\mathrm{Al}$ & $\mathrm{Be}$ & 30 & 73 & 0.046 & 0.038 & 84 & 75 \\
\hline A1 & $\mathrm{Fe}$ & 30 & 73 & 0.073 & 0.065 & 86 & 83 \\
\hline Al & $\mathrm{Au}$ & 30 & 73 & 0.093 & 0.092 & 86 & 85 \\
\hline Al & $B$ & 12 & 14.5 & 0.0324 & 0.0339 & 13.9 & 14.2 \\
\hline $\mathrm{A} 1$ & B & 12 & 7.3 & 0.0166 & 0.0163 & 7.3 & 7.6 \\
\hline $\mathrm{A} 1$ & B & 12 & 4.4 & 0.0098 & 0.0096 & 4.4 & 4.6 \\
\hline $\mathrm{A} 1$ & B & 12 & 3.4 & 0.0075 & 0.0074 & 3.4 & 3.6 \\
\hline A1 & B & 4 & 14.5 & 0.407 & 0.351 & 16.5 & 14.6 \\
\hline $\mathrm{Al}$ & B & 4 & 7.3 & 0.182 & 0.164 & 8.0 & 7.0 \\
\hline A1 & B & 4 & 4.4 & 0.109 & 0.095 & 5.0 & 4.3 \\
\hline $\mathrm{A} 1$ & B & 4 & 3.4 & 0.084 & 0.072 & 3.9 & 3.3 \\
\hline $\mathrm{Cu}-\mathrm{K} \alpha$ & $Z=10$ & 30 & 83 & 0.063 & 0.062 & 84 & 72 \\
\hline $\mathrm{Cu}-\mathrm{K} \alpha$ & $\mathrm{Au}$ & 30 & 83 & 0.104 & 0.092 & 94 & 85 \\
\hline $\mathrm{Cu}-\mathrm{L} \alpha$ & $Z=10$ & 10 & 83 & 0.433 & 0.452 & 80 & 71 \\
\hline $\mathrm{Cu}-\mathrm{L} \alpha$ & $\mathrm{Au}$ & 10 & 83 & 0.590 & 0.662 & 73 & 82 \\
\hline $\mathrm{Ni}-\mathrm{L} \alpha$ & $Z=10$ & 6 & 48 & 0.612 & 0.596 & 49.5 & 45 \\
\hline $\mathrm{Ni}-\mathrm{L} \alpha$ & $Z=10$ & 12. & 48 & 0.232 & 0.214 & 51 & 43 \\
\hline$N i-L \alpha$ & $Z=10$ & 20 & 48 & 0.131 & 0.122 & 51 & 40 \\
\hline $\mathrm{Ni}-\mathrm{L} \alpha$ & $Z=10$ & 30 & 48 & 0.117 & 0.101 & 54 & 47 \\
\hline $\mathrm{Ni}-\mathrm{K} \alpha$ & $Z=10$ & 15 & 26 & 0.094 & 0.087 & 28 & 23 \\
\hline $\mathrm{N} i-\mathrm{K} \alpha$ & $Z=10$ & 18 & 26 & 0.053 & 0.053 & 26 & 23 \\
\hline $\mathrm{N} i-\mathrm{K} \alpha$ & $Z=10$ & 22 & 26 & 0.035 & 0.033 & 27 & 24 \\
\hline $\mathrm{Ni}-\mathrm{K} \alpha$ & $Z=10$ & 30 & 26 & 0.017 & 0.017 & 26 & 23 \\
\hline$A u-L \alpha$ & Pt & 30 & 77 & 0.122 & 0.088 & 105 & 94 \\
\hline $\mathrm{Au}-\mathrm{L} \alpha$ & $P t$ & 25 & 77 & 0.178 & 0.136 & 98 & 91 \\
\hline$A u-L \alpha$ & $\mathrm{Pt}$ & 30 & 193 & 0.276 & 0.243 & 215 & 198 \\
\hline$A u-L \alpha$ & $P t$ & 25 & 193 & 0.366 & 0.368 & 192 & 183 \\
\hline
\end{tabular}


Table II

Bishop and Poole: Thickness Measurement of Copper on Nickel Substrate [20] Compared with NBS Calculations (Nominal Cu thickness was $45 \mu \mathrm{g} / \mathrm{cm}^{2} ; \theta=0^{\circ}, \psi=75^{\circ}$ )

\begin{tabular}{|c|c|c|c|}
\hline $\mathrm{E}_{\mathrm{o}}(\mathrm{keV})$ & $\mathrm{k}_{\mathrm{Cu}}$ & ${ }^{2} \mathrm{~B}-\mathrm{P}$ & ${ }^{z}$ NBS \\
\hline 10.75 & 0.476 & 29 & 26 \\
\hline 12.88 & 0.263 & 31 & 35 \\
\hline 14.76 & 0.176 & 35 & 37 \\
\hline 17.86 & 0.105 & 37 & 38 \\
\hline 20.9 & 0.075 & 36 & 40 \\
\hline 26.0 & 0.045 & 35 & 39 \\
\hline 31.1 & 0.033 & 37 & 41 \\
\hline
\end{tabular}




\section{Table III}

Bishop and Poole: Thickness Measurement of $\mathrm{MgF}_{2}$ on Si Substrate [20] Compared with NBS Calculations

(Nominal $\mathrm{MgF}_{2}$ thickness was $30 \mu \mathrm{g} / \mathrm{cm}^{2} ; \mathrm{Mg}-\mathrm{K} \alpha$ monitored, $\theta=0^{\circ}, \psi=75^{\circ}$ )

\begin{tabular}{|c|c|c|c|}
\hline$E_{0}(\mathrm{keV})$ & $\mathrm{k}_{\mathrm{Mg}}$ & $z_{B-P}$ & ${ }^{z} \mathrm{NBS}$ \\
\hline 6.6 & 0.478 & 36 & 44 \\
\hline 8.7 & 0.263 & 35 & 40 \\
\hline 10.75 & 0.172 & 34 & 39 \\
\hline 13.34 & 0.109 & 31 & 36 \\
\hline 15.83 & 0.087 & 31 & 35 \\
\hline 20.9 & 0.061 & 27 & 34 \\
\hline 26.0 & 0.052 & 25 & 34 \\
\hline 31.1 & 0.044 & 20 & 32 \\
\hline
\end{tabular}




\section{Table IV}

Kyser-Murata: Analysis of Binary Films

[22]

$$
\begin{aligned}
& \mathrm{Co}-\mathrm{Pt} \text { on } \mathrm{SiO}_{2} \\
& \mathrm{Mn}-\mathrm{Bi} \text { on } \mathrm{SiO}_{2}
\end{aligned}
$$

Compared with NBS Calculations

\begin{tabular}{|c|c|c|c|c|c|}
\hline${ }^{\mathrm{k}_{\mathrm{Co}}}$ & $\mathrm{k}_{\mathrm{Pt}}$ & ${ }^{z_{K}-M}$ & ${ }^{2} \mathrm{NBS}$ & $\mathrm{C}_{\mathrm{Co}}^{\mathrm{K}-\mathrm{M}}$ & $\begin{array}{l}\mathrm{C}_{\mathrm{Co}}^{\mathrm{NBS}} \\
\end{array}$ \\
\hline 0.0177 & 0.0581 & 53 & $51^{\circ}$ & 0.185 & 0.199 \\
\hline 0.0305 & 0.114 & 89 & 99 & 0.163 & 0.197 \\
\hline 0.0148 & 0.0675 & 56 & 60 & 0.140 & 0.154 \\
\hline 0.0067 & 0.0465 & 40 & 41 & 0.101 & 0.103 \\
\hline $\mathrm{k}_{\mathrm{Mn}}$ & $\mathrm{k}_{\mathrm{Bi}}$ & ${ }^{z_{K}-M}$ & ${ }^{z} \mathrm{NBS}$ & $\mathrm{C}_{\mathrm{Mn}}^{\mathrm{K}-\mathrm{M}}$ & $\mathrm{C}_{\mathrm{Mn}}^{\mathrm{NBS}}$ \\
\hline--- & 0.0766 & 54 & 60 & 0 & 0 \\
\hline 0.0226 & 0.0515 & 53 & 59 & 0.255 & 0.263 \\
\hline 0.0293 & 0.0220 & 38 & 41 & 0.510 & 0.518 \\
\hline 0.0329 & 0.00668 & 29 & 30 & 0.800 & 0.796 \\
\hline 0.0610 & ---- & 42 & 41 & 1.0 & 1.0 \\
\hline
\end{tabular}

$$
\begin{gathered}
z \text {-values in } \mu \mathrm{g} / \mathrm{cm}^{2} \\
\theta=0^{\circ} \\
\psi=52.5^{\circ}
\end{gathered}
$$


Table V

Analysis of Au-Ag Alloys

\begin{tabular}{|c|c|c|c|c|}
\hline $\mathrm{E}_{\mathrm{o}}(\mathrm{keV})$ & ANALYTE & $\mathrm{C}_{\text {CERT. }}$ & $\mathrm{k}_{\text {MEAS. }}$ & $\mathrm{k}_{\text {CALC. (Eqn 28) }}$ \\
\hline 10 & $A g-L \alpha$ & 0.1996 & 0.191 & 0.187 \\
\hline 48.5 & $\mathrm{Ag}-\mathrm{L} \alpha$ & 0.1996 & 0.0795 & 0.0826 \\
\hline 10 & $A u-M \alpha$ & 0.8005 & 0.771 & 0.784 \\
\hline 48.5 & $\mathrm{Au}-\mathrm{M} \alpha$ & 0.8005 & 0.755 & 0.753 \\
\hline 10 & $\mathrm{Ag}-\mathrm{L} \alpha$ & 0.3992 & 0.386 & 0.374 \\
\hline 48.5 & $\mathrm{Ag}-\mathrm{L} \alpha$ & 0.3992 & 0.181 & 0.184 \\
\hline 10 & $\mathrm{Au}-\mathrm{M} \alpha$ & 0.6005 & 0.559 & 0.569 \\
\hline 48.5 & $\mathrm{Au}-\mathrm{M} \alpha$ & 0.6005 & 0.537 & 0.532 \\
\hline 10 & $A g-L \alpha$ & 0.5993 & 0.584 & 0.575 \\
\hline 48.5 & $\mathrm{Ag}-\mathrm{L} \alpha$ & 0.5993 & 0.339 & 0.341 \\
\hline 10 & $\mathrm{Au}-\mathrm{M} \alpha$ & 0.4003 & 0.362 & 0.369 \\
\hline 48.5 & $A u-M \alpha$ & 0.4003 & 0.337 & 0.334 \\
\hline 10 & $\mathrm{Ag}-\mathrm{L} \alpha$ & 0.7758 & 0.764 & 0.758 \\
\hline 48.5 & $\mathrm{Ag}-\mathrm{L} \alpha$ & 0.7758 & 0.542 & 0.550 \\
\hline 10 & $\mathrm{Au}-\mathrm{M} \alpha$ & 0.2243 & 0.201 & 0.202 \\
\hline 48.5 & $A u-M \alpha$ & 0.22 .43 & 0.181 & 0.177 \\
\hline
\end{tabular}


Table VI

Analysis of $\mathrm{Au}-\mathrm{Cu}$ Alloys

\begin{tabular}{|c|c|c|c|c|}
\hline $\mathrm{E}_{\mathrm{O}}(\mathrm{keV})$ & ANALYTE & ${ }_{\text {CERT. }}$ & $k_{\text {MEAS. }}$ & $\mathrm{k}_{\text {CALC. }}($ Eqn 28) \\
\hline 10 & $\mathrm{Cu}-\mathrm{L} \alpha$ & 0.1983 & 0.166 & 0.153 \\
\hline 40 & $\mathrm{Cu}-\mathrm{L} \alpha$ & 0.1983 & 0.067 & 0.074 \\
\hline 10 & $A u-M \alpha$ & 0.8015 & 0.749 & 0.756 \\
\hline 40 & $\mathrm{Au}-\mathrm{M} \alpha$ & 0.8015 & 0.675 & 0.676 \\
\hline 10 & $\mathrm{Cu}-\mathrm{L} \alpha$ & 0.3964 & 0.354 & 0.327 \\
\hline 40 & $\mathrm{Cu}-\mathrm{L} \alpha$ & 0.3964 & 0.166 & 0.179 \\
\hline 10 & $\mathrm{Au}-\mathrm{M} \alpha$ & 0.6036 & 0.531 & 0.538 \\
\hline 40 & $\mathrm{Au}-\mathrm{M} \alpha$ & 0.6036 & 0.428 & 0.436 \\
\hline 10 & $\mathrm{Cu}-\mathrm{L} \alpha$ & 0.5992 & 0.557 & 0.529 \\
\hline 40 & $\mathrm{Cu}-\mathrm{L} \alpha$ & 0.5992 & 0.313 & 0.336 \\
\hline 10 & $A u-M \alpha$ & 0.4010 & 0.332 & 0.336 \\
\hline 40 & $A u-M \alpha$ & 0.4010 & 0.253 & 0.246 \\
\hline 10 & $\mathrm{Cu}-\mathrm{L} \alpha$ & 0.7985 & 0.764 & 0.752 \\
\hline 40 & $\mathrm{Cu}-\mathrm{L} \alpha$ & 0.7985 & 0.550 & 0.576 \\
\hline 10 & $\mathrm{Au}-\mathrm{M} \alpha$ & 0.2012 & 0.154 & 0.157 \\
\hline 40 & $\mathrm{Au}-\mathrm{M} \alpha$ & 0.2012 & 0.110 & 0.104 \\
\hline
\end{tabular}




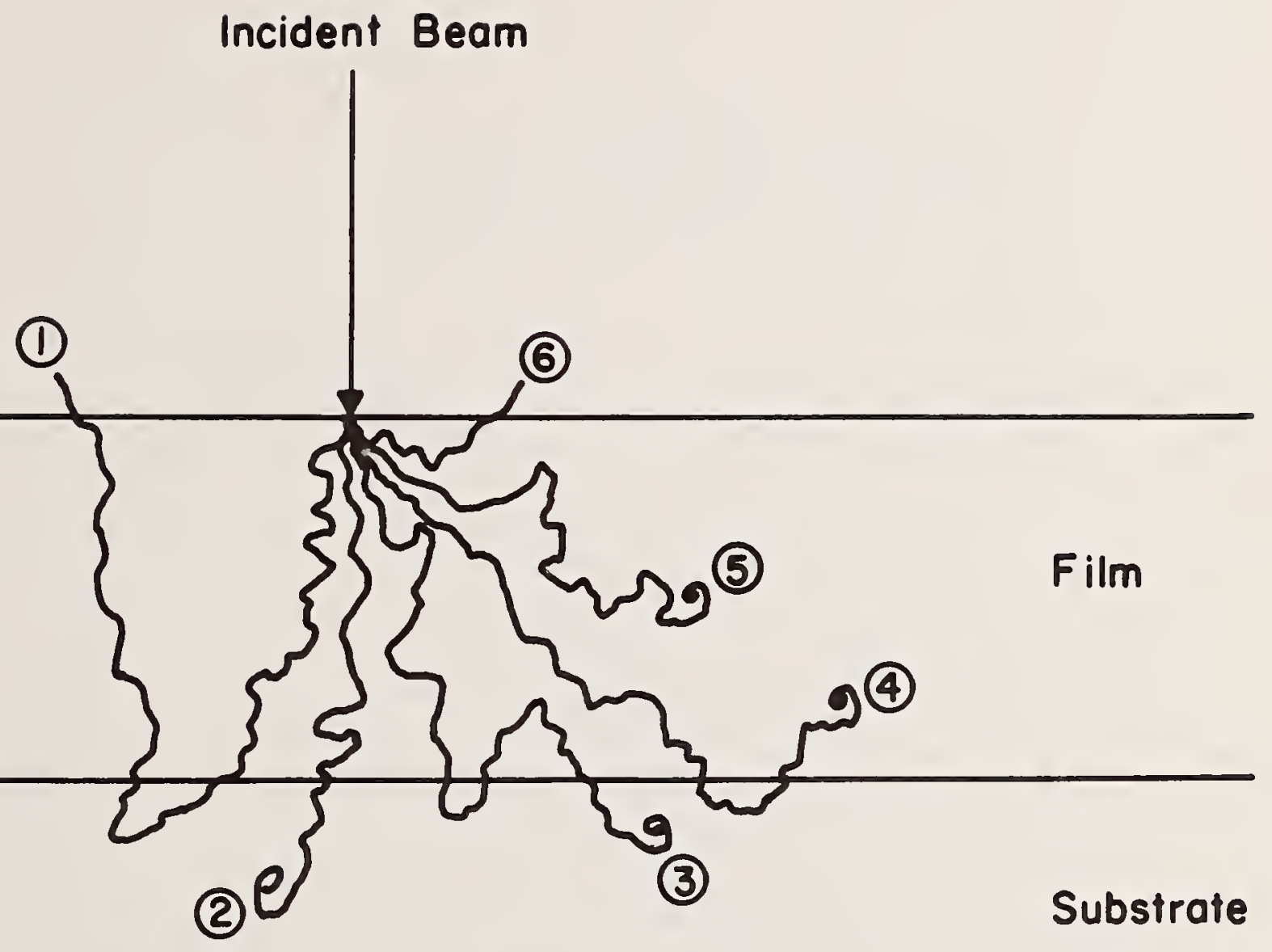

Fig. (1) Schematic representation of possible electron trajectories in a thin film-substrate target. 



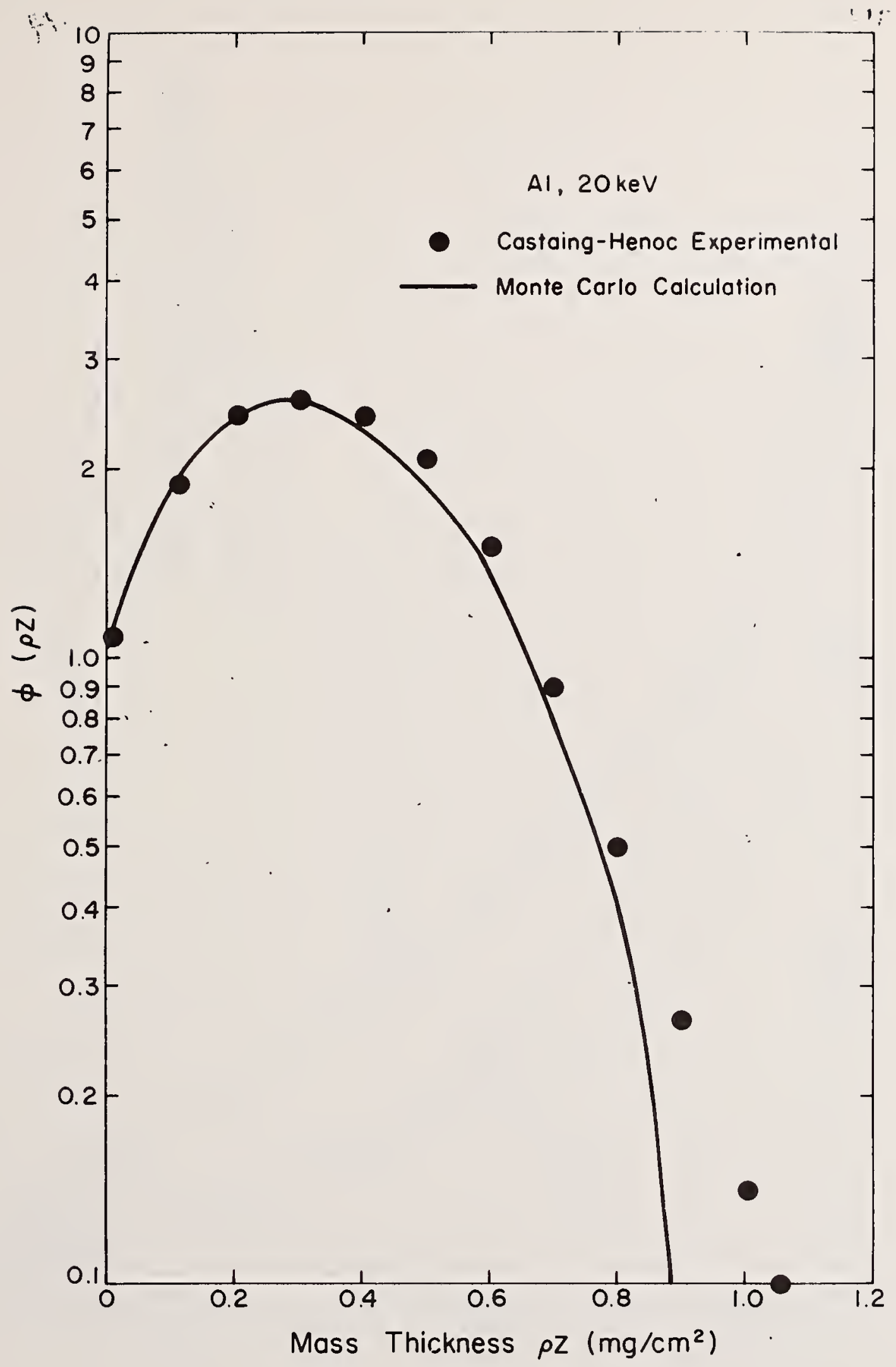

Fig. (2) Function $\phi(z)$ plotted against $z$ for aluminum target bombarded normally by $20 \mathrm{keV}$ electrons. After Castaing and Henoc [12]. 
5 


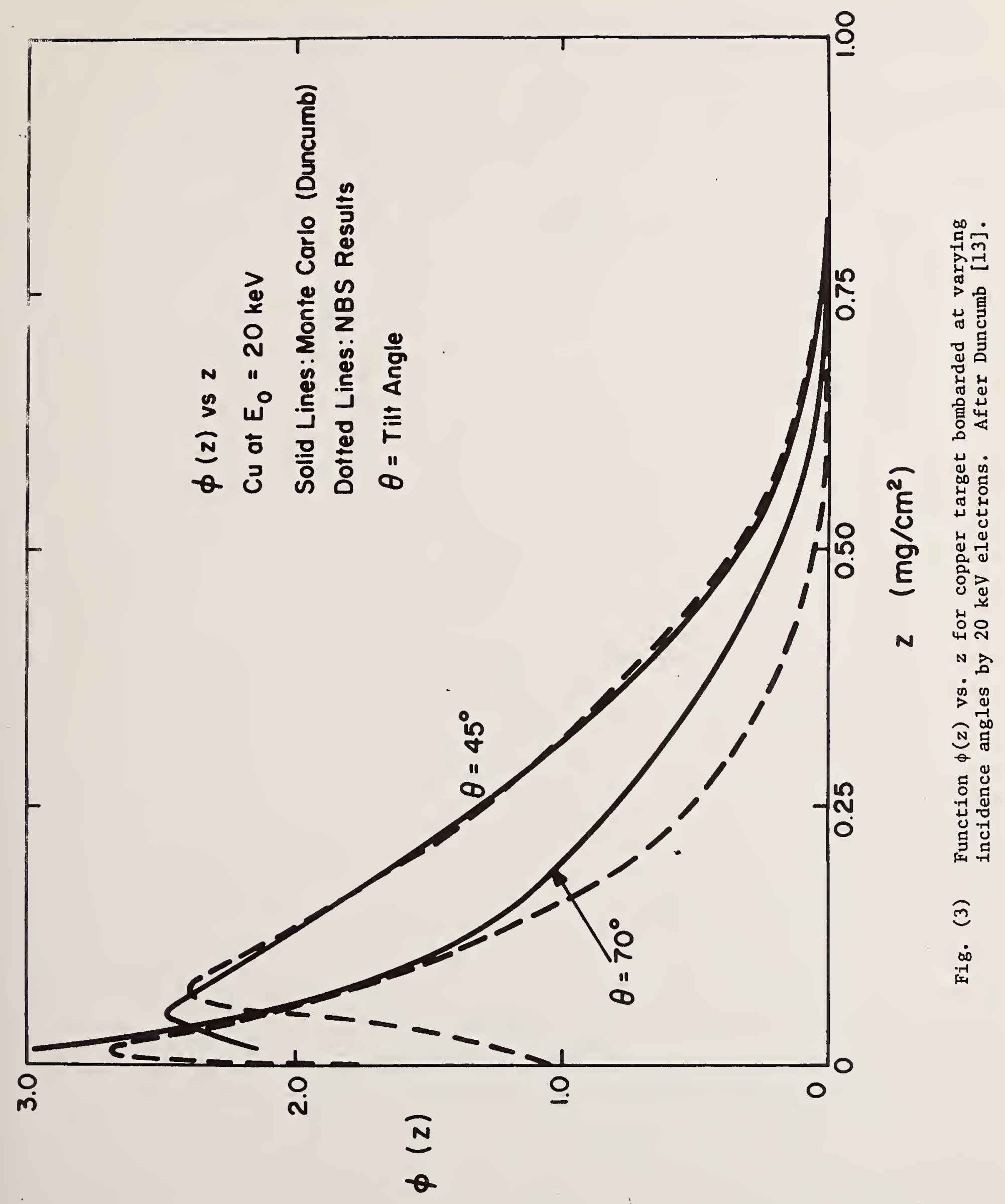





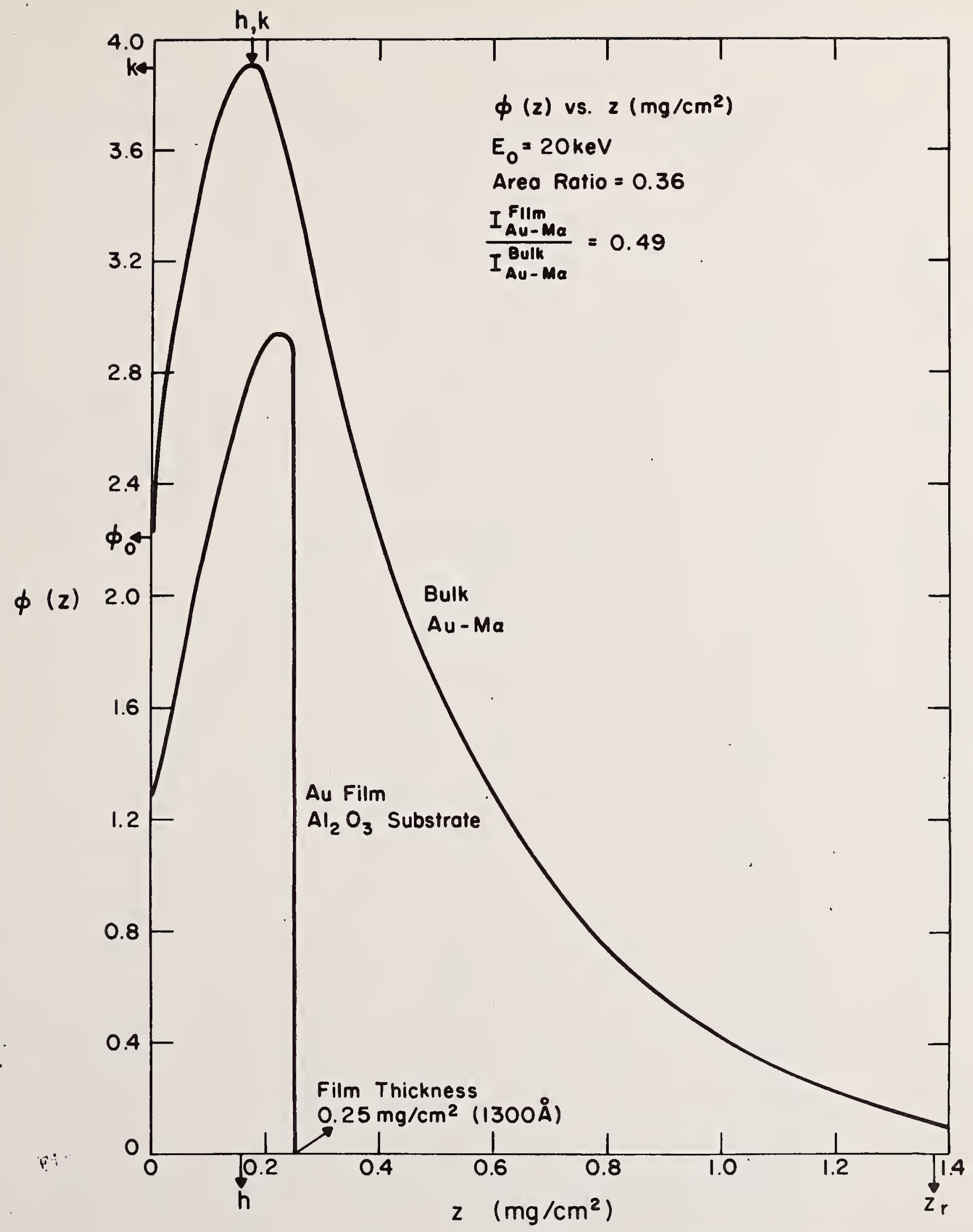

F1g. (4) Function $\phi(z)$ vs, $z$ for $A u-M \alpha$ radiation from solid gold and from $300 \AA$ thick gold on $\mathrm{Al}_{2} \mathrm{O}_{3}$ substrate bombarded normally by $20 \mathrm{keV}$ electrons. The parameters $\phi_{0}, \mathrm{~h}, \mathrm{k}$, and $\mathrm{z}_{\mathrm{r}}$ (see text) are indicated. 



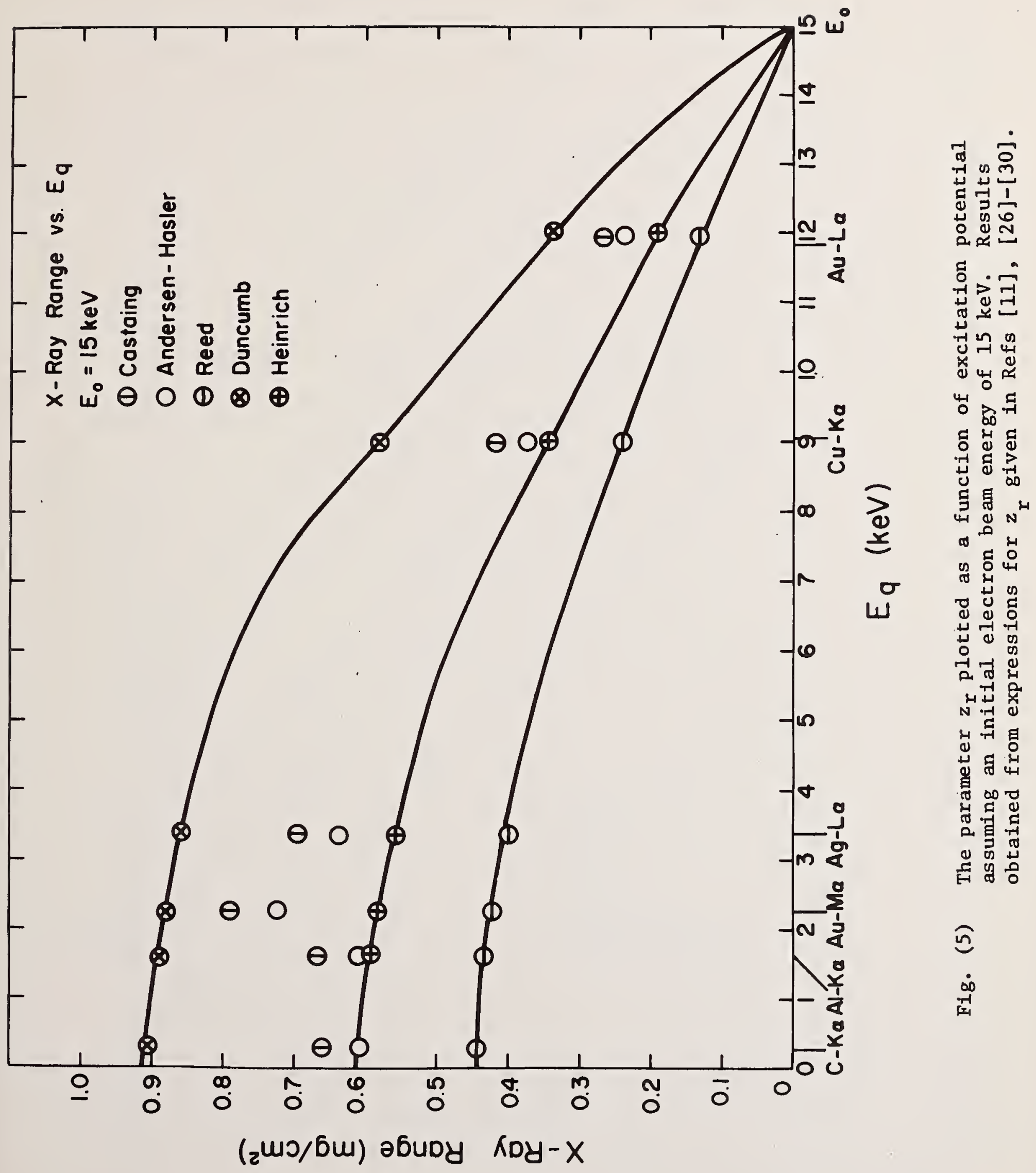





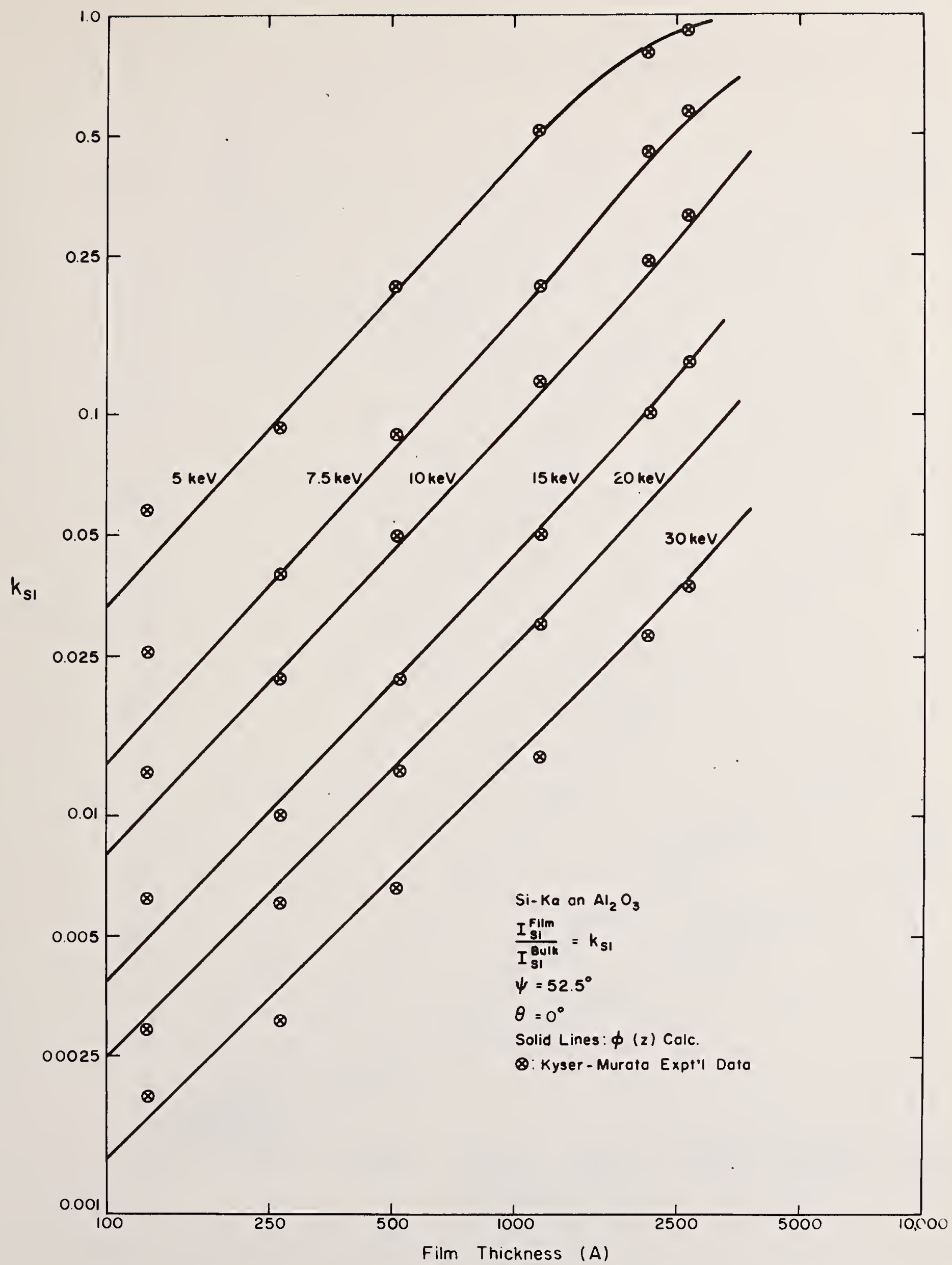

Fig. (6) Comparison of analytic model with experimental results taken from Kyser and Murata [22].

(6a) Results for $\mathrm{Si}$ films on $\mathrm{Al}_{2} \mathrm{O}_{3}$ substrate. 



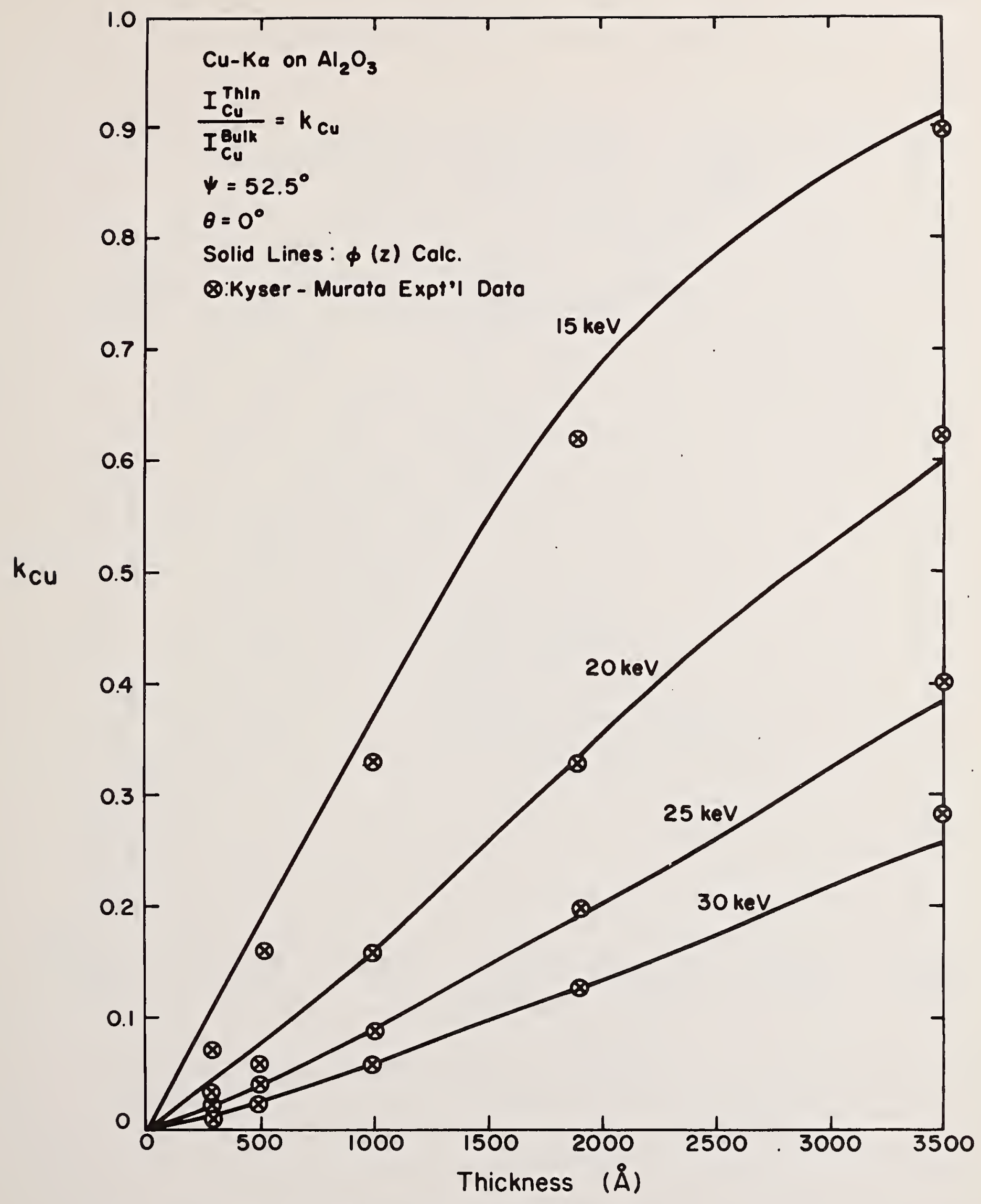

(6b) Results for Cu films on $\mathrm{Al}_{2} \mathrm{O}_{3}$ substrate. 



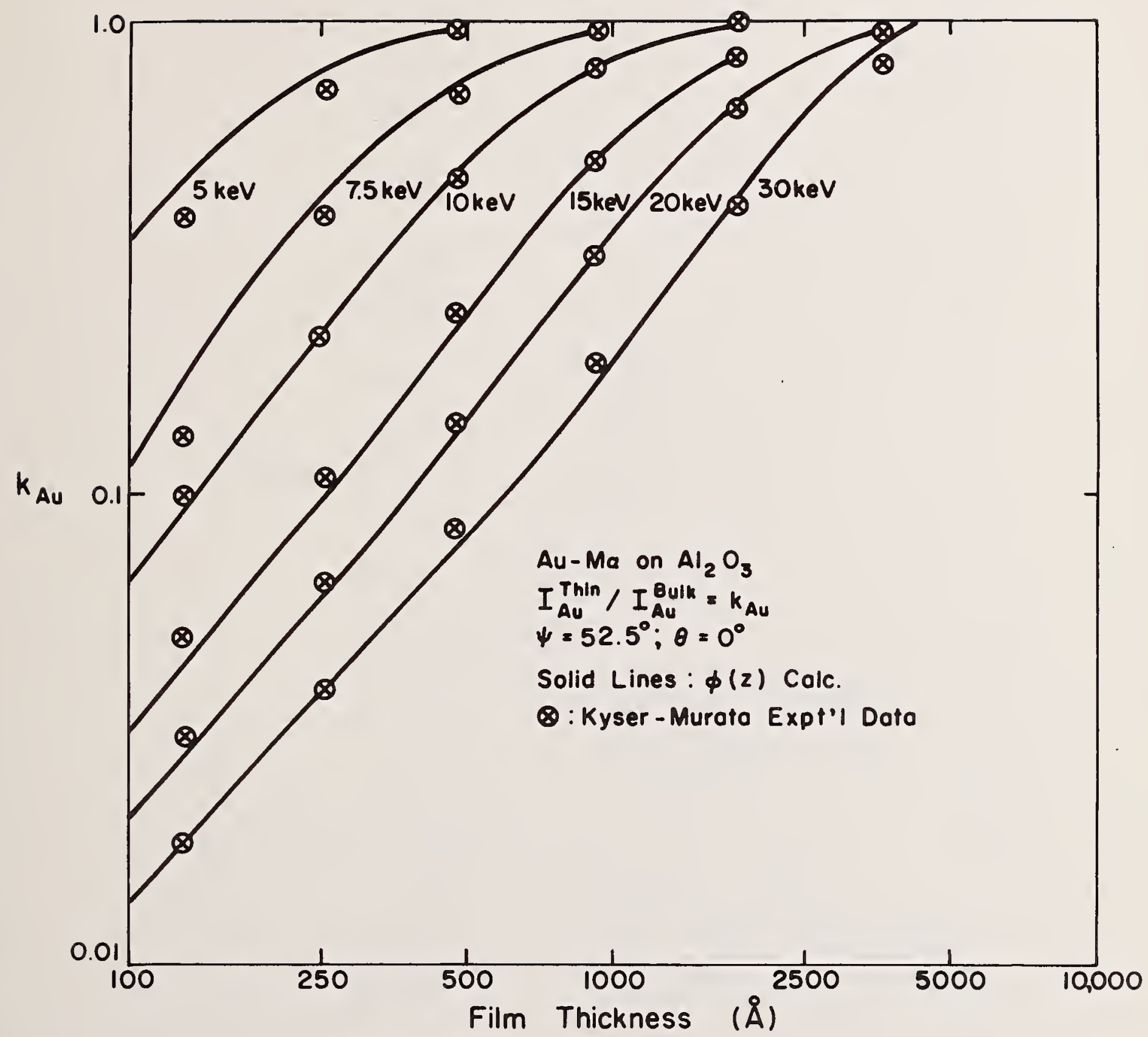

(6c) Results for Au films on $\mathrm{Al}_{2} \mathrm{O}_{3}$ substrate. 



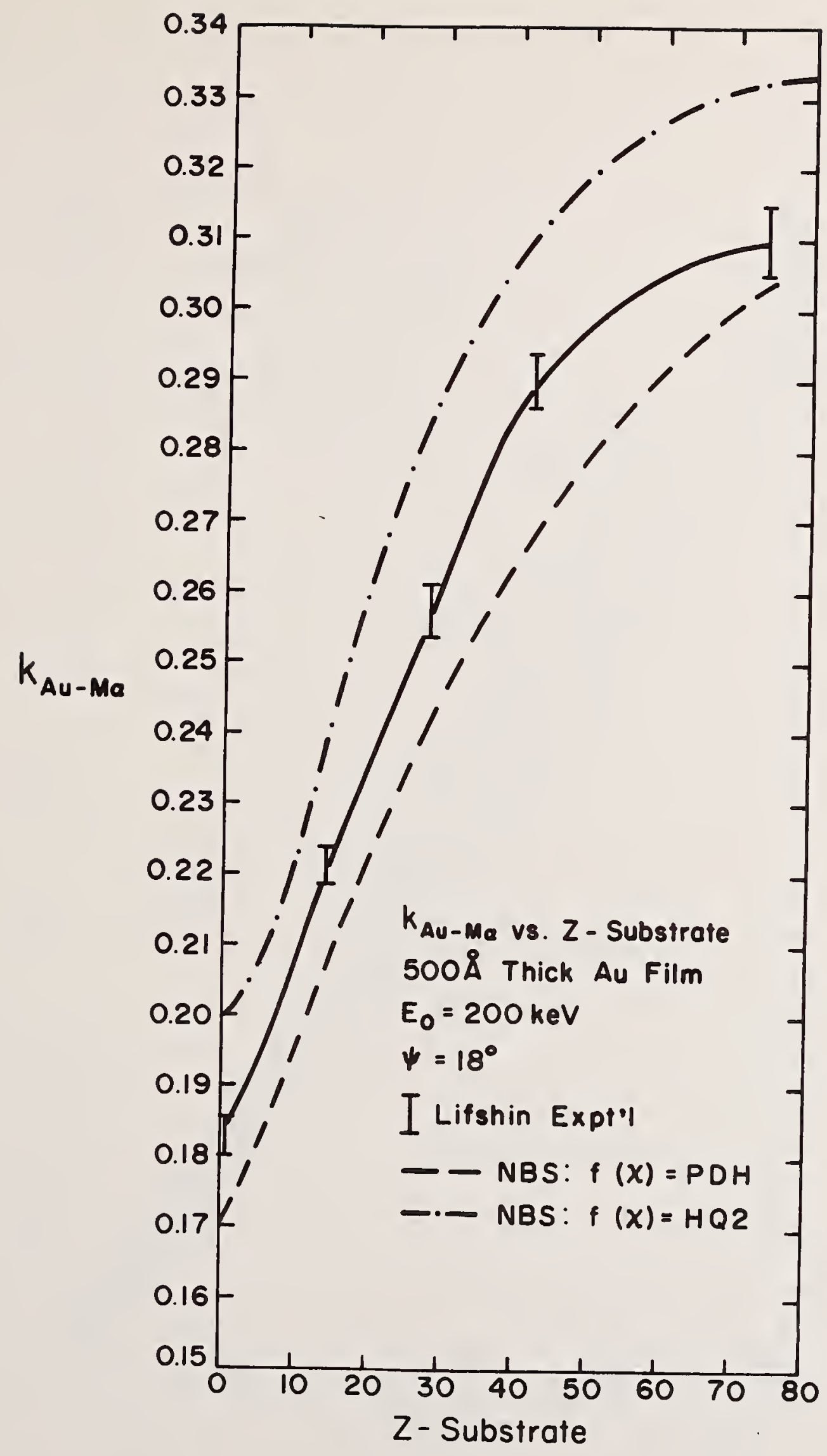

Fig. (7) The intensity ratio of Au-Ma X-rays from a $500 \AA$ thick gold film to Au-Ma from a solid block of gold as a function of substrate atomic number. Experimental data from Bolon and Lifshin [21]. Data calculated using two absorption correction expressions, Eqn (1) and the Philibert-Duncumb-Heinrich relation (see Ref [8]). 



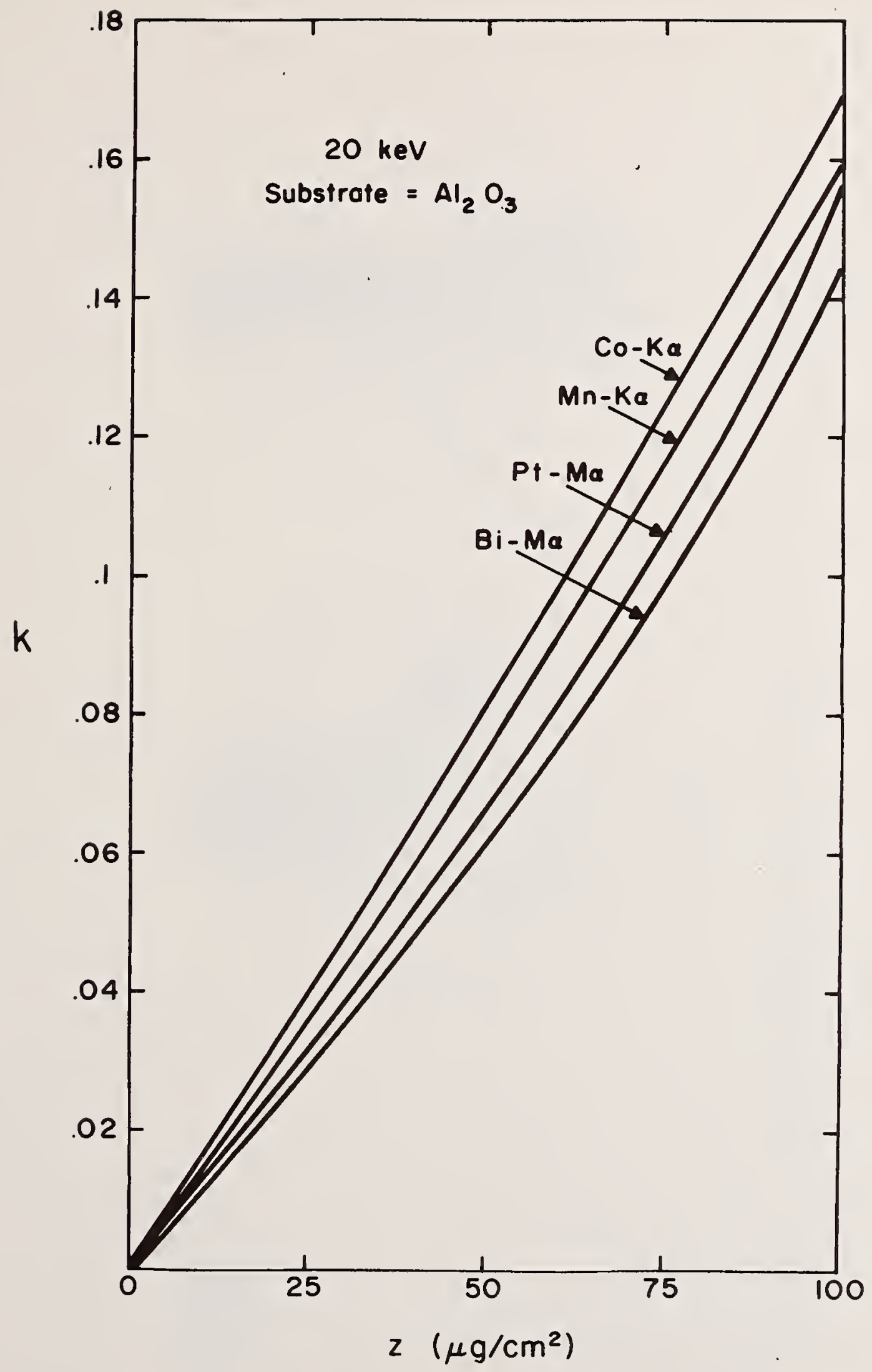

Fig. (8) Calculated intensity ratios as a function of thickness for films of fo, $\mathrm{Pt}, \mathrm{Mn}$, and $\mathrm{Bi}$ against the pure solid element. Specimens bombarded normally by $20 \mathrm{keV}$ electrons, $\psi=52.5^{\circ}$. This figure can be used to calculate the results given in Table IV. 


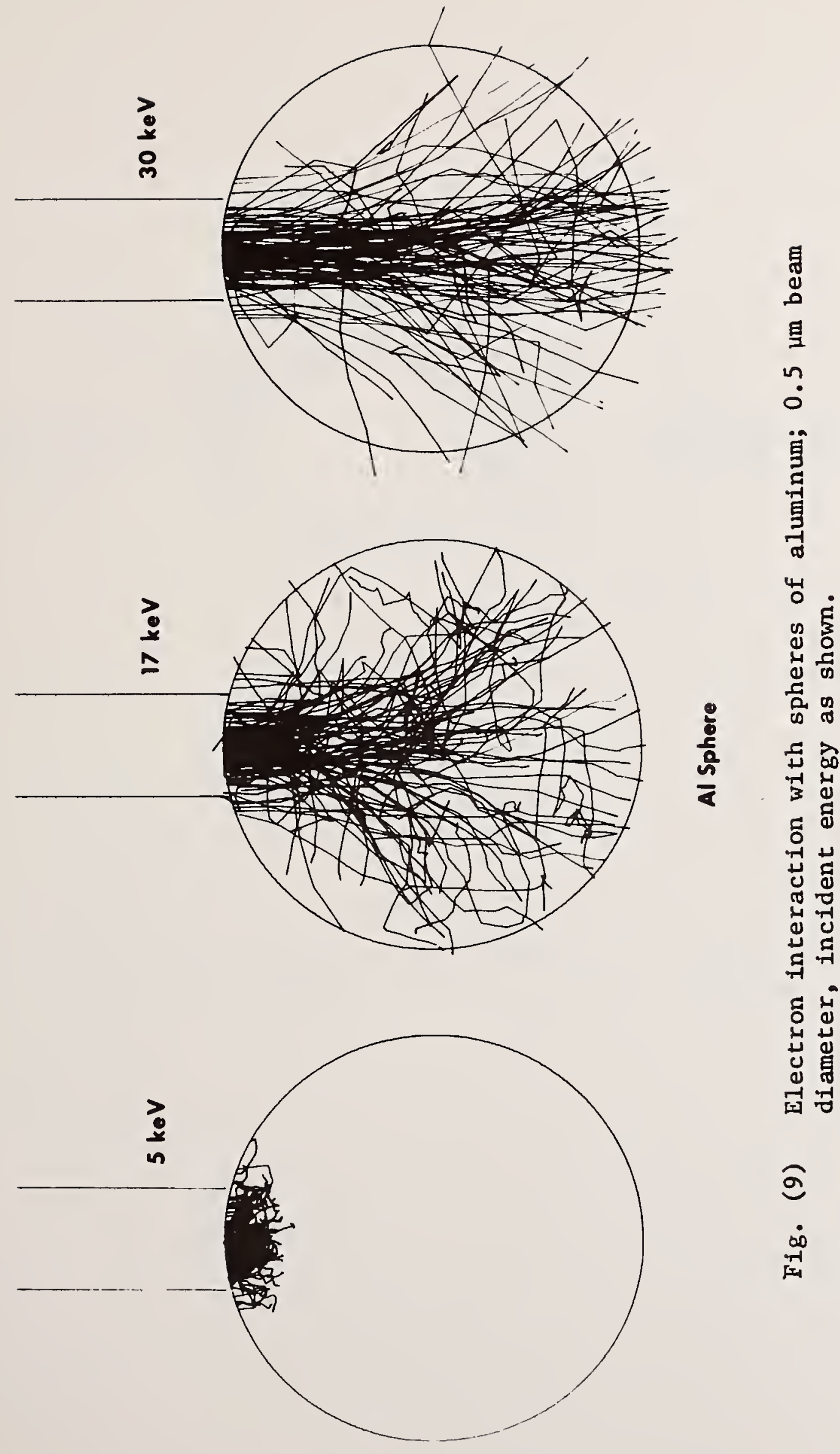



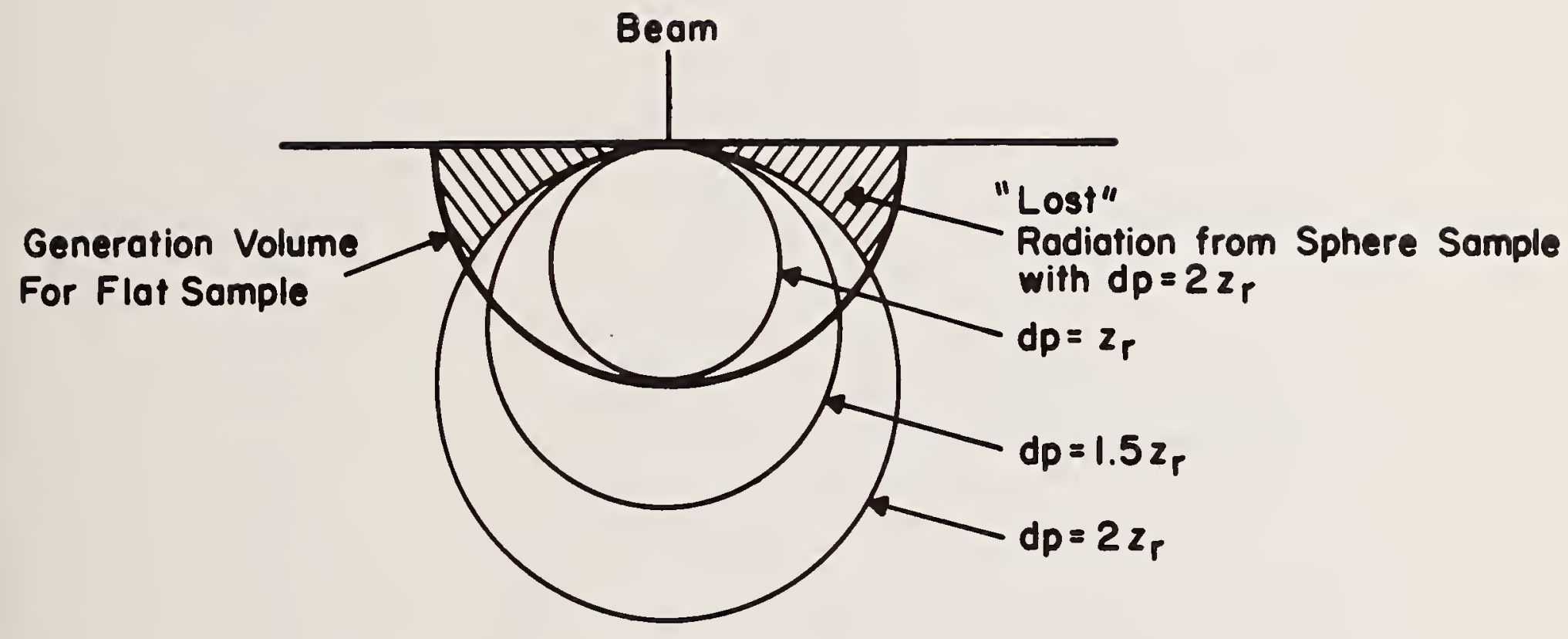

Fig. (10) Schematic representation of "missing" X-ray emission due to particle geometry. 



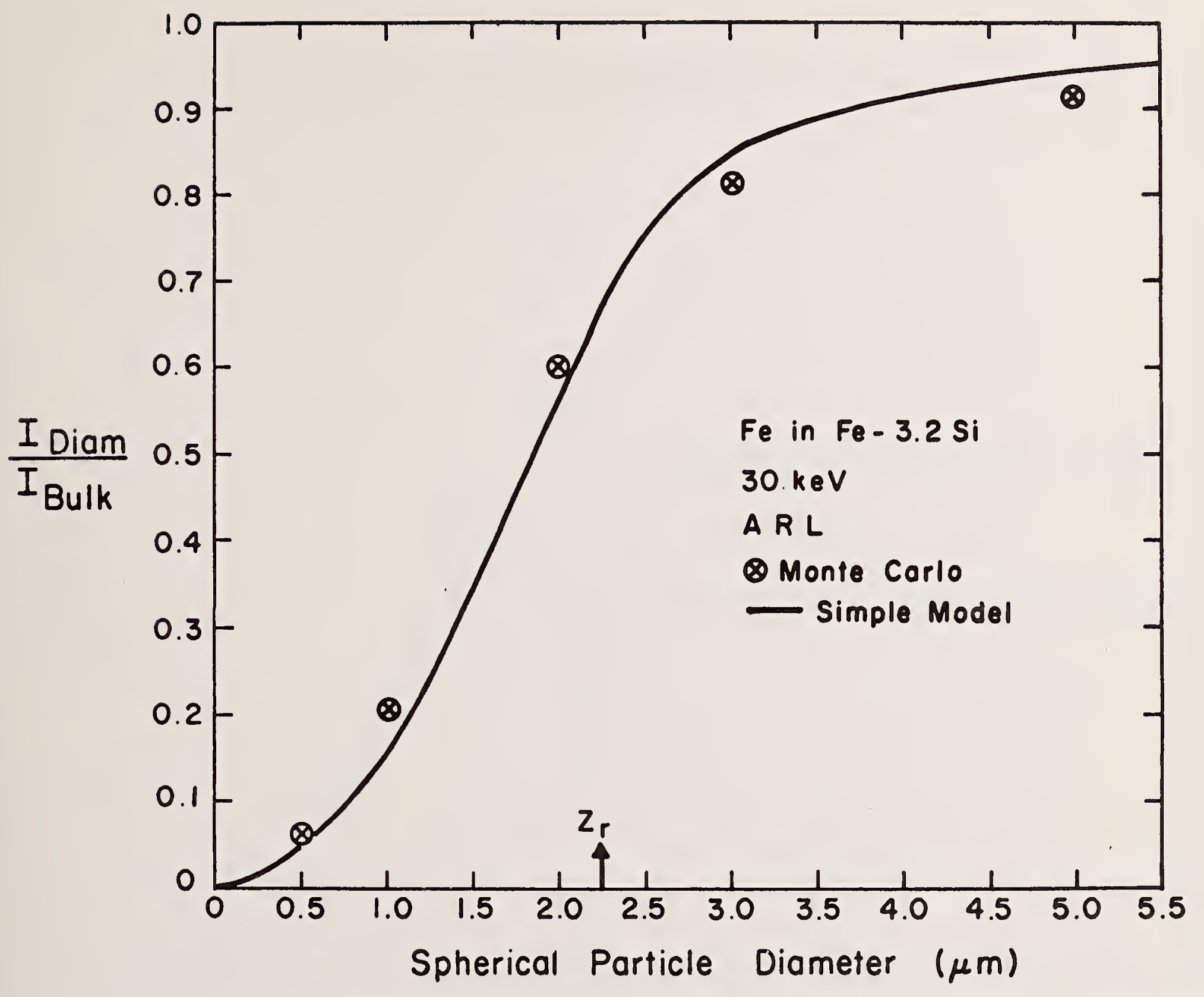

Fig. (11) Results of calculation for Fe-Si spheres. 



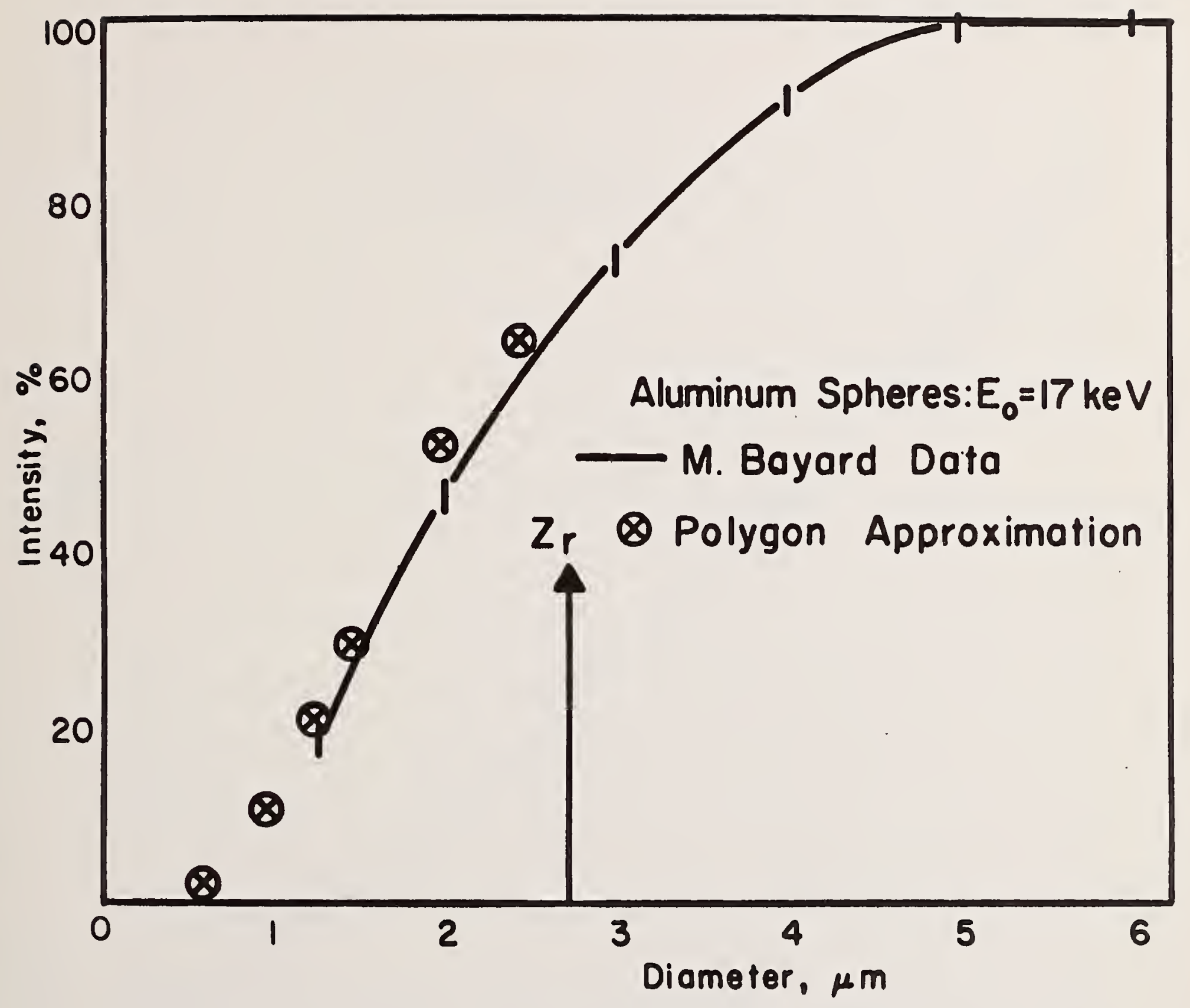

Fig. (12) Results of calculation for Al spheres as compared with experimental data of Bayard [60]. 



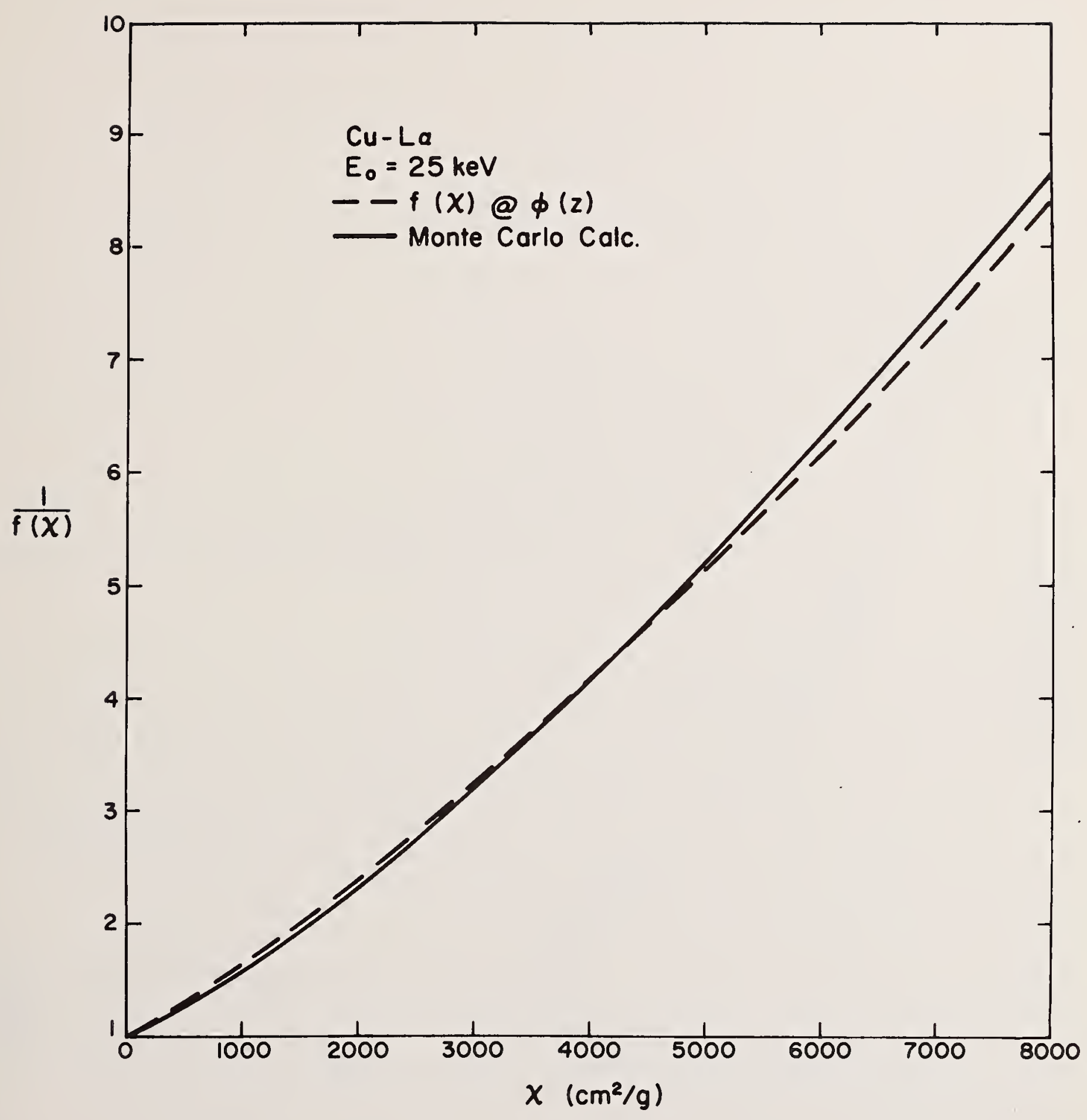

Fig. (13) Parameter $1 / f(X)$ vs. $X$ for $C u-L \alpha$; Eo $=25 \mathrm{keV}$. 



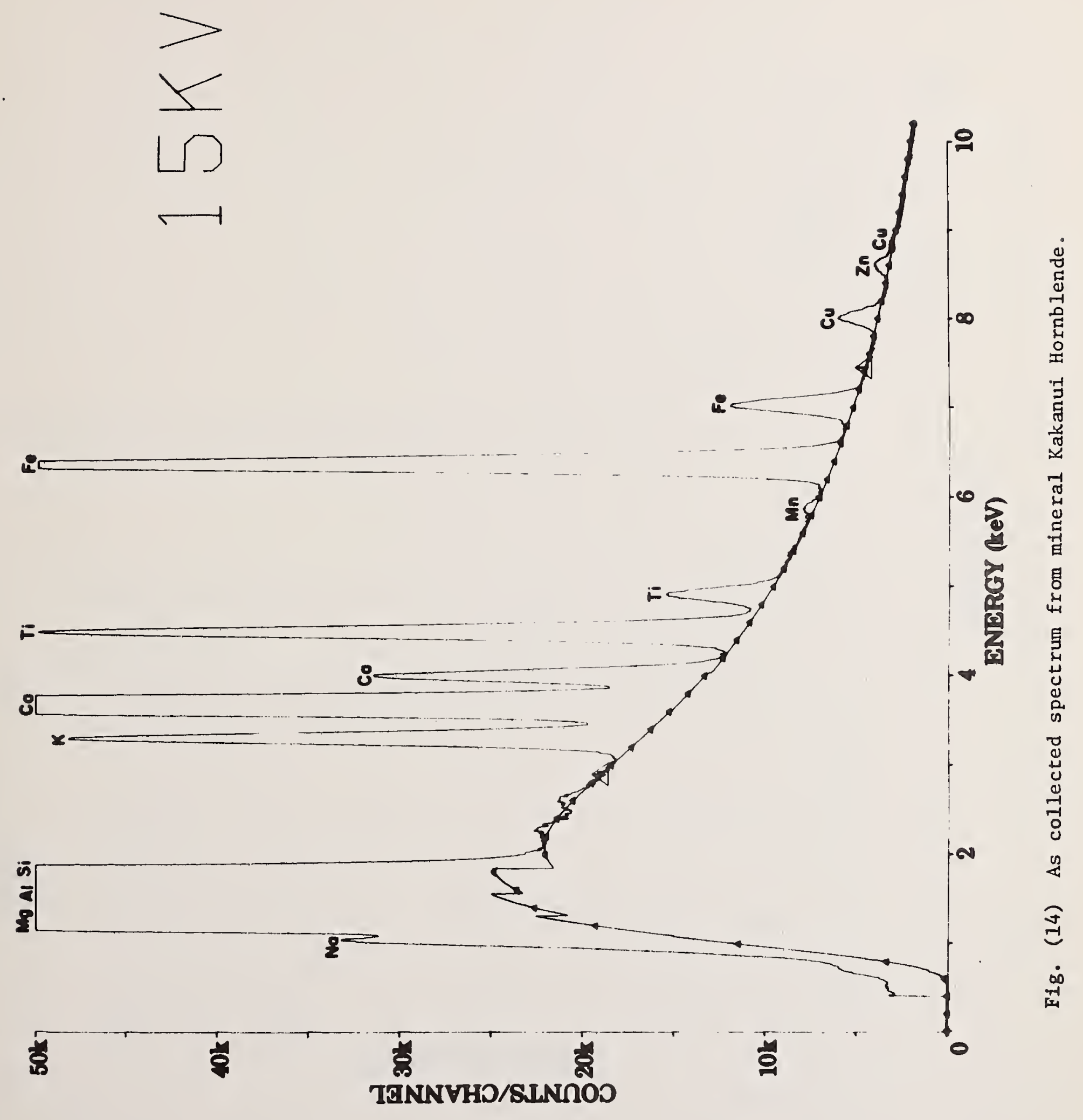





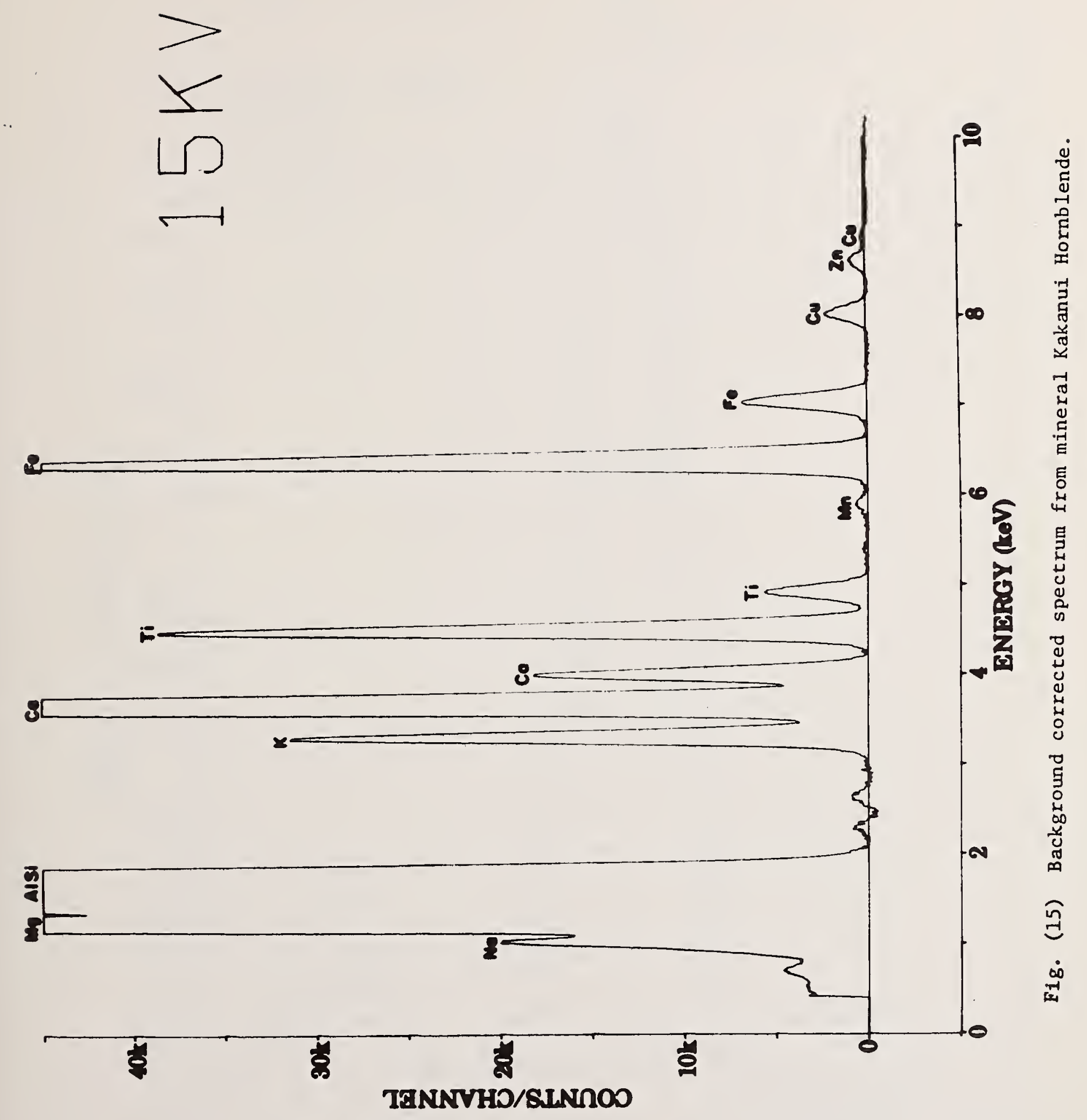





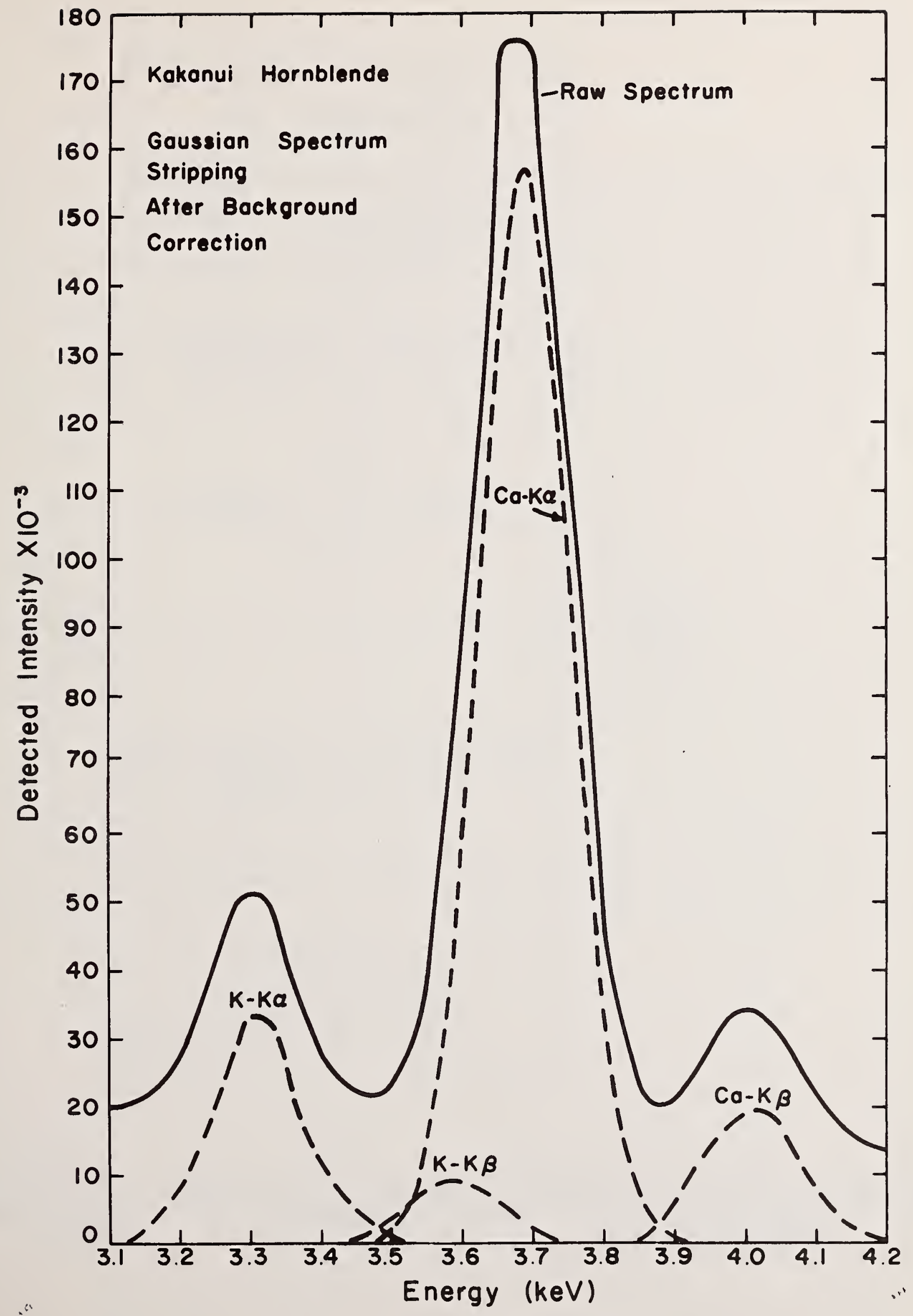

F1g. (16) Spectrum analysis of selected energy region from Fig. (15). 



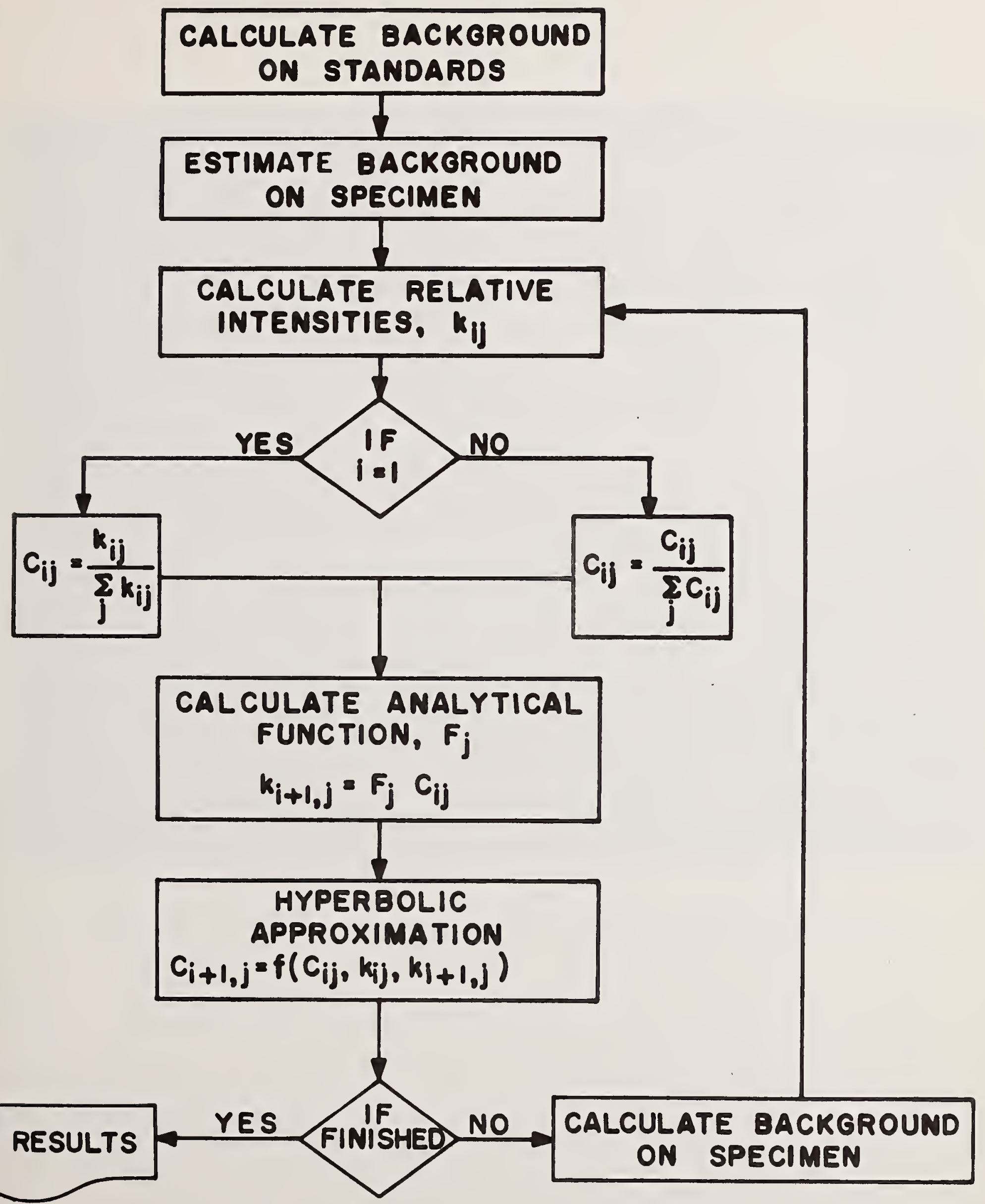

Fig. (17) Flow diagram of program FRAME B. 



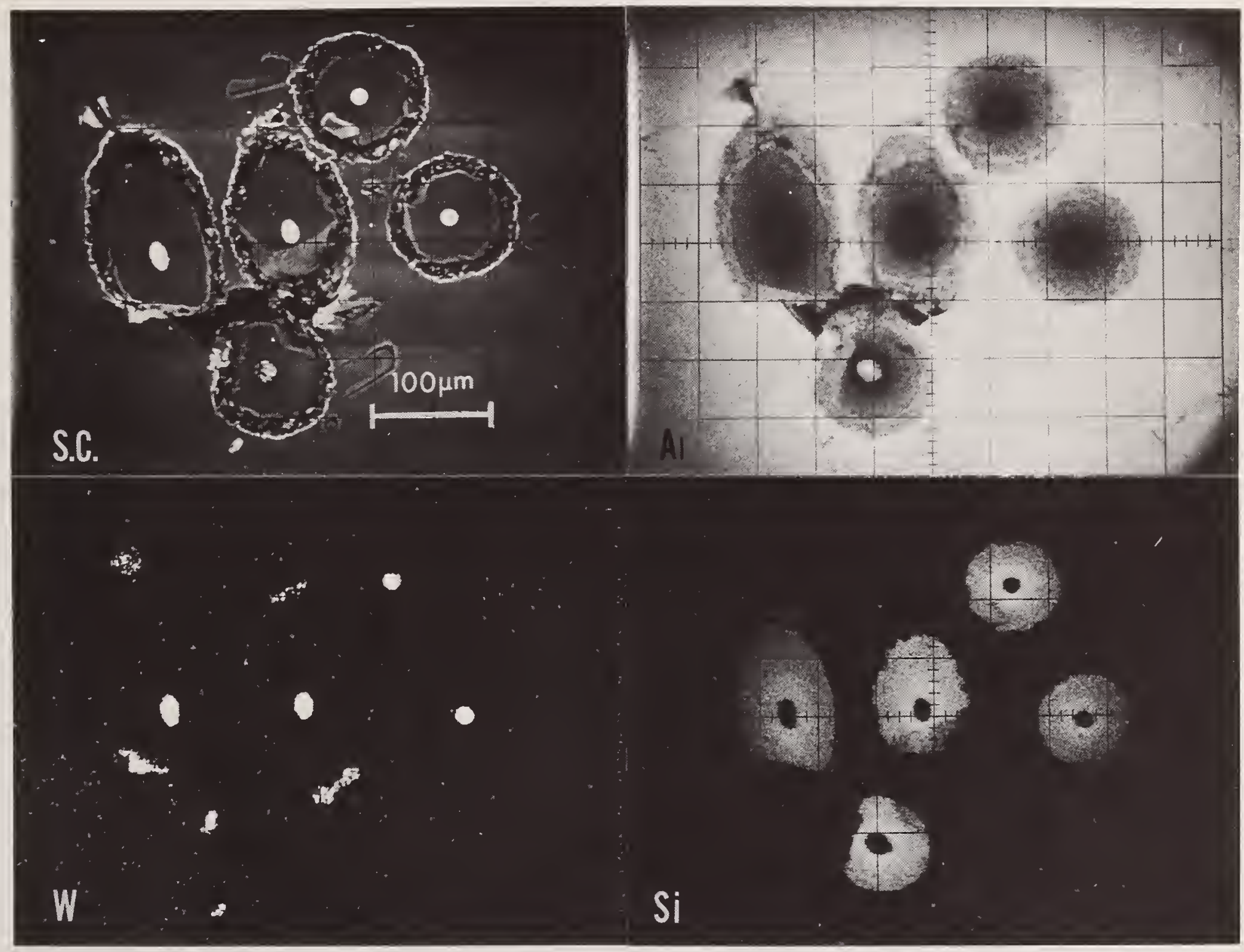

Fig. (18) Phase locations in SiC reenforced-Al matrix composite. 

Task 4

Melt Shape in Weightless Crystal Growth

\author{
S. R. Corie11, S. C. Hardy \\ Metallurgy Division \\ Institute for Materials Research \\ and \\ M. R. Cordes \\ Applied Mathematics Division \\ Institute for Basic Standards
}

Summary

Experimental and theoretical investigations of the floating zone process of crystal growth and purification have been carried out with particular emphasis on microgravity conditions. The shape and stability of a vertical rotating liquid zone subject to a vertical gravitational field have been calculated numerically. For a cylindrical volume and a weak gravitational field, analytic results have been obtained for the shape and stability of a zone for arbitrary direction of the gravitational field. Comparison of the numerical results with experiments on vertical water zones between two metal rods indicate that the liquid zone is slightly less stable than predicted theoretically. 
The floating zone process is widely used to purify materials and to grow single crystals ${ }^{1}$. For example, in recent years several hundred tons per year of silicon are prepared by this process ${ }^{2}$. One of its advantages is that the melt is not in contact with a crucible and contamination problems are minimized. In the earth's gravitational field there is apparently a maximum zone length (approximately $1.5 \mathrm{~cm}$ for silicon) above which the zone is unstable ${ }^{1,3}$ (i.e., the liquid zone fails). In microgravity zones of greater length can be stable. The shape of the liquid zone, in particular, the angle the liquid-vapor interface makes with the solid-vapor interface at the freezing interface, determines the shape of the resulting solid ${ }^{3-6}$. Thus, the shape of the zone is important for growing crystals of constant diameter.

We have carried out theoretical investigations of the shape and stability of liquid zones, including both numerical and analytical calculations. In addition, we have experimentally measured liquid zone shape and stability to compare with the predictions of theory.

\section{Theory}

As illustrated in Fig. 1, we consider a liquid zone of length L between two solids which are circular cylinders and are coaxial. The radii of the upper and lower solids are $R_{u}$ and $R$, respectively. Defining the usual cylindrical coordinate system $(r, \theta, z)$ with origin midway between the two solids and coaxial with them, we study the effects of a constant gravitational acceleration $g$ along the negative $z$-axis and of rotation with constant angular velocity $\Omega$ about the $z$-axis on the shape and stability of the liquid zone. For example, in the experiments to be described later, water zones 
between steel end plates were studied. In the studies of floating liquid zones in simulated zero gravity by Carruthers and Grasso ${ }^{7,8}$, the solids were glass and the liquid was a water-ethanol solution which was surrounded by nujol (a high molecular weight mineral oil). In a typical floating zone process the solids might be silicon and the liquid molten silicon. However, the present theory assumes that the liquid is either static or uniformly rotating. Thus, in applying the results of the calculations to the floating zone process, the effect of fluid flow in the melt is neglected. The error due to this approximation is undetermined at present and requires additional theoretical and experimental work.

We assume that the 1iquid-vapor interface is axisymmetric and denote the shape by the function $r(z)$. This assumption is consistent with the direction of the gravitational field and the axis of rotation. (In the Appendix we obtain some approximate analytic results for the case where the gravitational field is at an arbitrary angle to the liquid zone, and the 1iquid shape is not axisymmetric.) Since the liquid is static (or uniformly rotating), the energy $E$ of the liquid depends only on the shape $r(z)$ of the liquid-vapor interface. The energy is composed of gravitational, rotational, and surface contributions. In addition, there is the constraint that the liquid volume remain constant (we assume constant liquid density). Application of the calculus of variations ${ }^{9}$ to this problem yields differential equations which govern the shape and stability of the liquid. Since this procedure has been previously described ${ }^{10}$ and a similar calculation is given in an Appendix, we will simply state the basic equations. The requirement that the first variation $\delta E$ vanish gives the Euler equation for $r(z)$, i.e., $\gamma\left\{r^{-1}\left(1+r_{z}{ }^{2}\right)^{-\frac{1}{2}}-r_{z z}\left(1+r_{z}{ }^{2}\right)^{-3 / 2}\right\}=-\rho g z+\left(\rho \Omega^{2} r^{2} / 2\right)+p$ 
where $\gamma$ is liquid-vapor surface energy, the subscript $z$ denotes differentiation, i.e., $r_{z} \equiv(\mathrm{dr} / \mathrm{dz}), \rho$ is density difference between liquid and vapor (although we will use the word vapor for the fluid surrounding the liquid zone, it should be recognized that the calculation is valid for the vapor replaced by any fluid which is immiscible with the liquid zone), and $\mathrm{p}$ is a Lagrange multiplier (independent of $z$ ) which results from the volume constraint, i.e.,

$$
V=\int_{-L / 2}^{L / 2} \pi r^{2} d z=\text { CONSTANT }
$$

The left-hand side of Eq. (1) is the product of surface tension and the mean curvature of the interface. Thus, the equation is the familar Laplace equation which gives the pressure difference across a curved interface. The equation can be written in dimensionless form by introducing the variables $n=z / \alpha, \psi=r / \alpha, \varepsilon=\rho g \alpha^{2} / \gamma, \omega=\rho \Omega^{2} \alpha^{3} / 2 \gamma$, and $P=\alpha p / \gamma$, where $\alpha$ is an arbitrary length (to correspond with our previous calculations we take $\alpha=R /\left[1+\varepsilon_{R}\right]^{\frac{1}{2}}$ where $\left.\varepsilon_{R}=\rho \Omega^{2} R^{3} / \gamma\right)$. The differential equation for $\psi(\eta)$ is then $\psi^{-1}\left(1+\psi_{\eta}{ }^{2}\right)^{-\frac{1}{2}}-\psi_{\eta \eta}\left(1+\psi_{\eta}{ }^{2}\right)^{-3 / 2}=-\varepsilon \eta+\omega \psi^{2}+P$

where $\psi_{\eta}=(\mathrm{d} \psi / \mathrm{d} \eta)$. In this form it is apparent that the shape of the liquid zone is determined by the two dimensionless parameters $\varepsilon$ and $\omega$. If the shape $r(z)$ of the zone satisfies the Euler or Laplace equation, then the energy is stationary with respect to small variations in shape. In order to determine whether the energy is a minimum and the liquid zone stable with respect to small variations in the shape one must consider the second variation. If the second variation $\delta^{2} \mathrm{E}$ is positive then the energy 
is a minimum. From the calculus of variations ${ }^{9}$ in order for $\delta^{2} \mathrm{E}$ to be positive, the Legendre condition is necessary and the Jacobi differential equation must possess certain properties. For our problem the Legendre condition is always satisfied ${ }^{10}$. The Jacobi differential equation is

$[\mathrm{d} / \mathrm{dz}]\left[\gamma \mathrm{rW} /\left(1+\mathrm{r}_{\mathrm{z}}\right)^{3 / 2}\right]+\left[\rho \Omega^{2} \mathrm{r}^{2}+\gamma / \mathrm{r}\left(1+\mathrm{r}_{\mathrm{z}}{ }^{2}\right)^{\frac{1}{2}}\right] \mathrm{W}=-\mu \mathrm{r} / 2$

where $W=\delta r$ and $\mu$ is a Lagrange multiplier used to satisfy the volume constraint on $W$, viz.,

$$
\int_{-L / 2}^{L / 2} r W d z=0
$$

Consider a non-trivial $W$ which satisfies Eqs. (4) and (5) and the boundary condition $W(-L / 2)=0$. Since $r$ is positive, it follows from $E q$. (5) that $W$ must vanish once in the interval $(-\mathrm{L} / 2)<z<(\mathrm{L} / 2)$. The Jacobi condition which ensures stability $\left(\delta^{2} \mathrm{E}>0\right)$ requires that $W$ should not vanish again In the interval. Since $W$ satisfies a linear differential equation, the stability criterion may be written as

$$
z^{*}>(\mathrm{L} / 2)
$$

where $z *$ (called the conjugate point) satisfies the equation

$$
u\left(z^{*}\right) \int_{-L / 2}^{z^{*}} v r d z-v\left(z^{*}\right) \int_{-L / 2}^{z^{*}} u r d z=0
$$

and is the first root of this equation greater than $(-\mathrm{L} / 2)$. The functions $u(z)$ and $v(z)$ are non-trivial solutions of the homogeneous $(\mu=0)$ and inhomogeneous $(\mu \neq 0)$ Jacobi equation which vanish at $z=(-L / 2)$. Since $z *$ is independent of the choice of $\mu, u_{z}(-L / 2)$ and $v_{z}(-L / 2)$, any convenient value of these quantities may be used. Thus, the shape and stability of a 
liquid zone are determined by the solutions of the Euler and Jacobi equations, respectively.

We discuss the appropriate boundary conditions for $r(z)$, the zone shape. If the trijunction (intersection of solid, liquid, and vapor phases) does not occur at a corner of the solid, as illustrated in Fig. 2, then the balance of forces in the direction tangential to the solid requires that

$$
\gamma_{\mathrm{SL}}+\gamma_{\mathrm{LV}} \cos \theta_{\mathrm{L}}^{*}=\gamma_{\mathrm{SV}}
$$

where $\gamma_{S L}, \gamma_{L V}$, and $\gamma_{S V}$ are the solid-liquid, liquid-vapor, and solid-vapor surface energies, respectively, and $\theta_{L}^{*}$ is the angle between the solid-liquid and liquid-vapor interface measured in the liquid. Since for given substances and conditions the surface tensions are fixed, this equation specifies $\theta_{\mathrm{L}}^{*}$ at the trijunction. For a liquid between two solids without corners, the slope $r_{z}$ of the liquid shape is then specified at the trijunctions. If the trijunction occurs at a corner in the solid as illustrated in Fig. 2, the following inequalities must be satisfied ${ }^{11}$

$$
\text { either } \gamma_{\mathrm{SL}}-\gamma_{\mathrm{SV}} \geq \gamma_{\mathrm{LV}} \cos \theta_{\mathrm{V}} \text { or } \theta_{\mathrm{V}}>\pi
$$

and either $\gamma_{S V}-\gamma_{S L} \geq \gamma_{L V} \cos \theta_{L}$ or $\theta_{L}>\pi$

where $\theta_{V}$ and $\theta_{L}$ are defined in Fig. 2. The above inequalities are equivalent to

$$
\theta_{\mathrm{L}}^{*} \leq \theta_{\mathrm{L}} \leq \pi-\theta_{\mathrm{S}}+\theta_{\mathrm{L}}^{*}
$$

where $\theta_{S}=2 \pi-\left(\theta_{L}+\theta_{V}\right)$ and $\cos \theta_{L}^{*}=\left(\gamma_{S V}-\gamma_{S L}\right) / \gamma_{L V}$. Note that for $\theta_{S}=\pi$, the inequalities demand that $\theta_{\mathrm{L}}=\theta_{\mathrm{L}}^{*}$. For $\theta_{\mathrm{S}}<\pi$, there is a range of possible $\theta_{L}$ values. If one considers a rounded corner from a macroscopic viewpoint, an identical range of angles is possible. The appropriate boundary condition is that the liquj.d be at the corner. If as a result of a calculation of $r(z)$ one finds that $\theta_{L}$ does not satisfy the appropriate inequalities, then obviously such a liquid zone is unstable (i.e., the liquid will pull away 
from the corner) and the calculation should be repeated with different boundary conditions. Experimentally the range of allowed $\theta_{L}$ values can be increased by using composite solids, i.e., each edge is a different material. For example, Carruthers and Grasso ${ }^{7-8}$ coated the vertical surface of their glass with grease to prevent capillary run-away. In the floating zone process, the temperature gradients presumably prevent the liquid from moving along the solid-vapor interface. Thus, for the situation with corners shown in Fig. 1 , we will use the boundary conditions $r(-L / 2)=R$ and $r(L / 2)=R_{u}$. In other circumstances one might wish to specify $\mathrm{r}_{z}$ at one or both of the endpoints $(z= \pm L / 2)$ rather than $r$.

Since there is an unknown Lagrange multiplier $\mathrm{p}$ in the Euler equation, an additional condition on the liquid zone is required. In an experiment where given volume of liquid is placed between two inert solids, the additional condition is to specify the volume of liquid in the zone. For the floating zone process, one can also specify the volume in the zone. However, in order to grow a solid of uniform diameter, recent evidence ${ }^{4-6}$ indicates that the slope $\mathrm{r}_{\mathrm{z}}$ at the freezing interface must have a fixed value (e.g., arctan $\left(r_{z}\right) \cong 11$ degrees for silicon $\left.{ }^{6}\right)$. Thus, rather than specifying the volume, one could specify $\dot{r}_{z}$ at the freezing interface.

Numerical Calculations

An algorithm and computer program has been developed for solving the Euler equation (with boundary conditions $r(-\mathrm{L} / 2)=\mathrm{R}, r(\mathrm{~L} / 2)=\mathrm{R}_{\mathrm{u}}$, and the volume specified) to find the liquid shape $r(z)$ and the Jacobi equation to determine the conjugate point and thus the stability of the zone. Since the boundary conditions on the Jacobi equation specify the function and its derivative at $\mathrm{z}=(-\mathrm{L} / 2)$, i.e., an initial value problem, the solution of this equation presents no problems. The Euler equation with $r(-L / 2)=R$ 
and $\mathrm{r}(\mathrm{L} / 2)=\mathrm{R}_{\mathrm{u}}$ is a two point boundary-value problem with the additional complication that the Lagrange multiplier $\mathrm{p}$ is unknown and must be chosen to yield the correct zone volume which we denote by $\mathrm{V}^{\infty}$. We will solve the Euler equation as an initial value problem by guessing values of $\mathrm{r}_{\mathrm{z}}(-\mathrm{L} / 2)$ $\equiv \mathrm{s}$ and $\mathrm{p}$. In general, the values of $\mathrm{r}(\mathrm{L} / 2)$ and the volume obtained from our initial guess will not be the desired ones. An iteration procedure which allows us to find $s$ and $p$ such that $r(L / 2)=R_{u}$ and $V=V^{\infty}$ will now be described.

We denote the values of $s$ and $p$ at the $n^{\text {th }}$ iteration by $s^{n}$ and $p^{n}$, respectively. We assume that $r\left(L / 2, s^{n}, p^{n}\right)-R_{u} \equiv R_{u}^{n}-R_{u}$ and $V\left(s^{n}, p^{n}\right)$ - $\mathrm{V}^{\infty}$ are sufficiently small that we can expand in a Taylor series about $\left(s^{n}, p^{n}\right)$, retain only linear terms in the expansion, and equate the result to zero (Newton's method). This yields

$$
\begin{aligned}
& \left(s^{n+1}-s^{n}\right)\left(\partial R_{u}^{n} / \partial s\right)+\left(p^{n+1}-p^{n}\right)\left(\partial R_{u}^{n} / \partial p\right)=R_{u}-r\left(\frac{L}{2}, s^{n}, p^{n}\right) \\
& \left(s^{n+1}-s^{n}\right)(\partial V / \partial s)+\left(p^{n+1}-p^{n}\right)(\partial V / \partial p)=v^{\infty}-V\left(s^{n}, p^{n}\right) .
\end{aligned}
$$

Here $\left(\partial R_{u}^{n} / \partial s\right)=\partial r\left(L / 2, s^{n}, p^{n}\right) / \partial s,\left(\partial R_{u}^{n} / \partial p\right)=\partial r\left(L / 2, s^{n}, p^{n}\right) / \partial p$, $(\partial V / \partial s)=\partial V\left(s^{n}, p^{n}\right) / \partial s$, and $(\partial V / \partial p)=\partial V\left(s^{n}, p^{n}\right) / \partial p$. These equations can be solved to find $\mathrm{p}^{\mathrm{n}+1}$ and $\mathrm{s}^{\mathrm{n}+1}$. The required partial derivatives are calculated numerically by the following procedure. Since the operators $(\partial / \partial s)$ and $(\partial / \partial p)$ commute with $(d / d z)$, the functions $r_{s}=\partial r(z, s, p) / \partial s$ and $r_{p}=\partial r(z, s, p) / \partial p$ satisfy the differential equations

$$
\begin{aligned}
& r_{s z z}-r_{s z}\left(\partial f / \partial r_{z}\right)-r_{s}(\partial f / \partial r)=0 \\
& r_{p z z}-r_{p z}\left(\partial f / \partial r_{z}\right)-r_{p}(\partial f / \partial r)=(\partial f / \partial p) .
\end{aligned}
$$


Here the subscripts indicate differentiation and $f(r, r, p)=\left[\left(1+r_{z}{ }^{2}\right) / r\right]$ $+\left[1+r_{z}{ }^{2}\right]^{3 / 2}\left[(\rho g z / \gamma)-\left(\rho_{\Omega} 2 r^{2} / 2 \gamma\right)-(p / \gamma)\right]=r_{z z}$. The appropriate boundary conditions at $z=(-\mathrm{L} / 2)$ are $r_{s}=0, r_{s z}=1, r_{p}=0$, and $r_{p z}=0$. These are initial value problems which are solved numerically to obtain $r_{s}$ and $r_{p}$. The $(\partial V / \partial s)$ and $(\partial V / \partial p)$ are obtained by numerical evaluation of the integrals

$$
(\partial V / \partial s)=\int_{-L / 2}^{L / 2} 2 \pi r r s d z
$$

and

$$
(\partial V / \partial p)=\int_{-L / 2}^{L / 2} 2 \pi r r_{p} d z
$$

Equations (1), (2), and (12-15) can be written as a system of nine first order differential equations. The method used to solve the initial value problem is a variable order, variable step formulation of the classic Adams methods as implemented by $\mathrm{Gear}^{12}$. As previously described, Newton's method is used to find new values of $s$ and $P$ and the system of differential equations is solved again. This iteration is continued until the relative change in $p$ and $s$ is less than a certain value (usually $10^{-3}$ ). An absolute change criterion is used if $\mathrm{s}$ and $\mathrm{p}$ are close to zero.

The iteration procedure can fail for a number of reasons. Frequently $r_{z}$ becomes very large and the calculation fails. If $r_{z}$ remained finite, one could presumably solve this problem by allowing the step size to become very small. However, it is known ${ }^{13}$ that solutions of the Euler equation can be multi-valued and $\mathrm{r}_{z}$ can become infinite. Presumably to solve such problems it would be necessary to use a different coordinate system than 
the $(r, z)$ system; we have not attempted this. Computations with $r_{z} \cong$ 37 have been successfully performed.

It is difficult to make any general statements about the initial guesses for $s$ and $p$. For certain cases, the initial $s$ and $p$ can be quite far from the final values and the iteration method will work. In other cases, it is necessary that the initial choice be close to the final values. In this case one can find reasonable initial choices by slowly incrementing some parameter from a known solution, i.e., a primitive continuation procedure. For example, if a solution is known for zero gravity and one wants a solution for a large gravitational field, one can slowly increase the gravitational field using the values of $s$ and $p$ at the smaller gravitational field as the initial choice of $s$ and $p$ for the larger gravitational field.

\section{Numerical Results}

Before presenting the results of a number of calculations, we will describe in some detail a particular stability calculation. We take

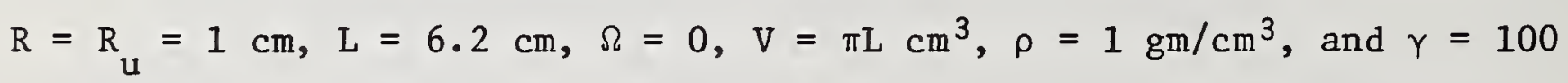
$\mathrm{mJ} / \mathrm{m}^{2} \quad$ (although we use these values, it should be realized that only certain dimensionless combinations of these quantities are important). We wish to find the maxirtum value of the gravitational acceleration for which the zone is stable (we denote this value by $g *$ and the corresponding $\varepsilon$ value by $\left.\varepsilon^{*}=\rho g * R^{2} / \gamma\right)$. It is evident that for $g=0$, a solution of Eq. 
is $r=R$ with $p=\gamma / R$. This provides initial values for $p$ and $s$, viz., $p=100$ and $s=0$. Since we know that for $L=2 \pi$, stability occurs at zero gravitational field we expect $\mathrm{g} *$ to be small. Upon carrying out calculations we find that for $g<g^{*}$, the iteration procedure works very well. For example, for $\mathrm{g}=0.05 \mathrm{~cm} / \mathrm{s}^{2}$, only four iterations are necessary to obtain a solution with $\mathrm{s}=0.041378$ and $\mathrm{p}=100.08$. However, for $g>g^{*}$, the iteration procedure fails. For example, for $\mathrm{g}=0.071 \mathrm{~cm} / \mathrm{s}^{2}$ and initial values of $s=0.075677$ and $p=100.27$ (which are the final values of $\mathrm{s}$ and $\mathrm{p}$ for $\mathrm{g}=.070 \mathrm{~cm} / \mathrm{s}^{2}$ ) after 10 iterations the relative change in $s$ for the next iteration is 0.25 . It is also apparent from the calculations that $\mathrm{s}$ is not converging toward a definite value but is oscillating; thus, it is unlikely that a greater number of iterations would lead to a solution.

Occasionally, large changes in $\mathrm{s}$ and $\mathrm{p}$ will occur and a solution of the Euler equation will be found which is unstable. For example, for $\mathrm{g}=0.074 \mathrm{~cm} / \mathrm{s}^{2}$ and initially $\mathrm{s}=0.075677$ and $\mathrm{p}=100.27$, a solution was found with $\mathrm{s}=-0.15319$ and $\mathrm{p}=101.21$. The conjugate point occurred at 2.848, which is less than $\mathrm{L} / 2$, which indicates that the solution was unstable. Physically, it was obvious that the solution was unstable since it bulged at the top and was constricted at the bottom. Using $s=$ -0.15319 and $p=101.21$ as initial values and changing $g$ one can find a 
family of unstable solutions, e.g., $s=-0.13340$ and $p=100.89$ yields a similar unstable solution for $g=0$. In general, the Euler equation with the boundary conditions $r(-L / 2)=R, r(L / 2)=R_{u}$ and fixed volume has a number of solutions. We have found solutions where $r_{z}=0$ for more than two values of $z$. Two solutions of the Euler equation are illustrated in Fig. 3. Both solutions satisfy the boundary conditions and have the same volume. The solid curve is the stable solution while the dashed curve is unstable. Physically, it is reasonable to assume that there is never more than one stable solution.

By approaching g* very slowly from below, it is possible to show that the failure of the iteration procedure corresponds to the solution of the Euler equation becoming unstable. For example for $g=0.07014,0.07017$ and $0.07020 \mathrm{~cm} / \mathrm{s}^{2}$, the conjugate point occurs at 3.102 , slightly greater than 3.100 and slightly less than 3.100, respectively ( a conjugate point less than 3.100 indicates instability). For $g=0.07020$ the magnitude of the relative change in $s$ and $p$ for the last iteration was 0.019 and 0.00011 , respectively. If the usual criterion (that the relative change be less than 0.001 ) had been used, the iteration process would have failed for $g=0.07017$ and 0.07020 and succeeded for $g=0.07014$. However, the solution for $g=0.07020$ satisfies the boundary conditions $r(L / 2)=1.0000$ 
and the volume constraint (e.g., $r_{z}(-L / 2)=0.078917$ and $r_{z}(L / 2)=$ 0.078915 ; these derivatives should be equal if $\mathrm{V}=\pi \mathrm{L}$ ).

In general, we will identify the failure of the iteration procedure as $g$ (or some other parameter) is slowly incremented with the onset of instability. This may slightly underestimate $g^{*}$; in the preceding example (requiring that the relative change in $\mathrm{s}$ and $\mathrm{p}$ be less than 0.001 ) we would take $g^{*}=0.07014$ whereas the solution is actually stable for $g=0.07017$. If $r_{z}$ is large, only a lower bound for $g^{*}$ can be obtained since it is difficult to tell whether the failure of the iteration process is due to instability or to large values of $\mathrm{r}_{z}$.

With a gravitational field present we have not been able to find in the unstable region a solution of the Euler equation which appears to be a continuation from the stable region of the stable solution (although solutions exist in the unstable region, they are qualitatively different from the stable solution). The basic question of whether the solution does not exist or whether the present numerical method is failing to find it is unresolved at present and requires further investigation. In zero gravity it is well known that such unstable solutions exist, e.g., $r=R$ for $L>2 \pi R$ (the present numerical program also calculates this rather trivial example).

A number of tests of the numerical calculations have been performed. We will describe a few of these. For $V=\pi R^{2} L, g=0, R_{u}=R$, and finite rotation, the computed conjugate point agrees with analytic results ${ }^{10}$. For $g=0, \Omega=0, R=R_{u}$, and $L / R=2$, we find that the smallest volume for which there is a stable zone is $\mathrm{V}=.387 \pi \mathrm{R}^{2} \mathrm{~L}$ (the iteration procedure fails below this volume); this appears to be in agreement with the 
analytic results of Gillette and Dyson ${ }^{14}$ (to the accuracy obtainable from their Fig. 6). For $R=R_{u}, V=\pi R^{2} L, \Omega=0$ and $\varepsilon$ small, we can compare the numerical solution for $r(z)$ with an approximate analytic solution (see reference 10 or the appendix). In Table 1, we compare the numerical values of $s$ and $p$ with the analytic values. For sufficiently small $\varepsilon$ there is agreement between the numerical and analytic results. How small an $\varepsilon$ is required is clearly a strong function of $\mathrm{L} / \mathrm{R}$. In fact, as $\varepsilon$ approaches $\varepsilon^{*}$ (where instability occurs; for (L/R) $=6.2, \varepsilon^{*}=7.02\left(10^{-4}\right)$ and for $\left.(\mathrm{L} / \mathrm{R})=5, \varepsilon^{*} \cong 0.073\right)$, the analytic and numerical results do not agree. This indicates that the analytic results are incorrect at $\varepsilon^{*}$ (even though $\varepsilon^{*}$ can be very small). Thus, the analytic prediction of the stability-instability demarcation will not be correct; this will be discussed subsequently.

The liquid zone shape depends on the five dimensionless parameters $\mathrm{R}_{\mathrm{u}} / \mathrm{R}, \mathrm{V} / \pi \mathrm{R}^{2} \mathrm{~L}, \mathrm{~L} / \mathrm{R}, \varepsilon$ and $\varepsilon_{\mathrm{R}}$. It is clear that with this many variables one cannot study all possible combinations. Our initial efforts have been to study the case $R_{u} / R=1, V / \pi R^{2} L=1, \varepsilon_{R}=0$ with $L / R$ and $\varepsilon$ variable. This allows comparison of our numerical results with our previous analytic studies for small $\varepsilon$ (see ref. 10 or the Appendix) and with the present experimental results and those of Carruthers and Grasso ${ }^{7-8}$. We have also carried out a small number of calculations with other values of the parameters.

For $R_{u}=R, V=\pi R^{2} L$, and $\varepsilon_{R}=0$, we have plotted in Figure 4 the maximum stable value of $L / 2 \pi R$ as a function of $\log \varepsilon$. For a given $\varepsilon$ the zone is stable if $L / 2 \pi R$ lies to the left of the curve and unstable if to 
Table 1

Comparison of Numerical and Analytic Values of $s$ and $p\left[V=\pi R^{2} L, R=R_{u}\right.$ and $\left.\Omega=0\right]$

\begin{tabular}{|c|c|c|c|c|c|}
\hline \multirow[b]{2}{*}{$(L / R)=6.2$} & \multirow[b]{2}{*}{$10^{4} \varepsilon$} & \multicolumn{2}{|c|}{ NUMER ICAL } & \multicolumn{2}{|c|}{ ANALYTIC } \\
\hline & & $10^{3} \mathrm{~s}$ & $\mathrm{p}$ & $10^{3} \mathrm{~s}$ & $\mathrm{p}$ \\
\hline \multirow{14}{*}{$(L / R)=5.0$} & 1 & 7.5575 & 100.00 & 7.5489 & 100.00 \\
\hline & 2.5 & 19.208 & 100.02 & 18.872 & 100.00 \\
\hline & 5 & 41.378 & 100.08 & 34.745 & 100.00 \\
\hline & 6 & 53.015 & 100.13 & 45.293 & 100.00 \\
\hline & 7 & 75.677 & 100.27 & 52.843 & 100.00 \\
\hline & 7.02 & 78.917 & $100 \cdot 30$ & 52.994 & 100.00 \\
\hline & $10^{4} \varepsilon$ & $10^{3} \mathrm{~s}$ & $\mathrm{P}$ & $10^{3} \mathrm{~s}$ & $\mathrm{p}$ \\
\hline & 10 & 4.3462 & 100.00 & 4.3466 & 100.00 \\
\hline & 20 & 8.6928 & 100.00 & 8.6932 & 100.00 \\
\hline & 50 & 21.745 & 100.01 & 21.733 & 100.00 \\
\hline & 100 & 43.570 & 100.03 & 43.466 & 100.00 \\
\hline & 200 & 87.811 & 100.11 & 86.932 & 100.00 \\
\hline & 500 & 234.11 & 100.83 & 217.33 & 100.01 \\
\hline & 700 & 376.62 & 102.47 & 304.26 & 100.01 \\
\hline
\end{tabular}


the right. For large values of $\varepsilon, r_{z}$ becomes large (e.g., for $\varepsilon=2.89$, $\left.\log \varepsilon=0.461, \mathrm{r}_{\mathrm{z}}(\mathrm{z}=-\mathrm{L} / 2)=23.6\right)$ and as previously indicated we may underestimate the stability of the zone. This is indicated by the dashed curve in Fig. 4; to the left of this curve the zone is certainly stable. As indicated previously, the analytic results for small $\varepsilon$ do not agree with the numerical results; a comparison and tabulation of the numerical results are given in Table 2. In the table the largest discrepancy between numerical and analytic results actually occurs for the smallest non-zero value of $\varepsilon$; at $L / R=6.24$ the ratio of the analytic $\varepsilon$ and the numerical $\varepsilon$ is 1.47 . This disagreement is not understood at present and the development of a valid analytic approximation requires further research. No values of the analytic $\varepsilon$ are given for $\varepsilon>0.025$ since there is no reason to expect agreement with the numerical results.

We briefly give the results of a few other calculations to illustrate the types of problems which can be investigated. For $R=R_{u},\left(V / \pi R^{2} L\right)=$ $0.5, L / R=2$ and $\varepsilon_{R}=0$, the maximum value of $\varepsilon$ for which the zone is stable is 1.537. For $R=R_{u} / 2,\left(V / \pi R^{2} L\right)=7 / 3$ (a conical volume), $L / R=$ 5 , and $\varepsilon=0$, the maximum value of $\varepsilon_{R}$ for which the zone is stable is 0.134. The zone shape for this case is illustrated in Fig. 1; the solid and dashed lines are for $\varepsilon_{R}=0$ and $\varepsilon_{R}=0.123$, respectively. For $R=$ $R_{u}, L / R=10$, and $\left(V / \pi R^{2} L\right)=16.667$ (a spherical volume, $V=\pi L^{3} / 6$ ), and $\varepsilon=0$, the maximum value of $\varepsilon_{R}$ for which the zone is stable is $\varepsilon_{R}=0.0361$. For this geometry the initial slopes are $3.89,29.1$, and 0.016 for the cases $\left(\varepsilon=0, \varepsilon_{R}=0\right),\left(\varepsilon=0.054, \varepsilon_{R}=0\right)$ and $\left(\varepsilon=0, \varepsilon_{R}=0.0361\right)$, respectively. 
TABLE 2

The Stability-Instability Demarcation:

Bond Number $\varepsilon$ versus Zone Length for

$R_{\mathrm{u}}=\mathrm{R}, \mathrm{V}=\pi \mathrm{R}^{2} \mathrm{~L}$ and $\varepsilon_{\mathrm{R}}=0$. Comparison of Numerical and Analytic Values.

\begin{tabular}{|c|l|l|}
\hline \multirow{2}{*}{$\mathrm{L} / \mathrm{R}$} & \multicolumn{2}{|c|}{$\varepsilon$} \\
\cline { 2 - 3 } & NUMERICAL & ANALYTIC \\
\hline $2 \pi$ & 0.0 & 0.0 \\
6.24 & 0.000258 & 0.000380 \\
6.20 & 0.000702 & 0.00102 \\
6.10 & 0.00239 & 0.00332 \\
5.92 & 0.00723 & 0.00927 \\
5.85 & 0.0100 & 0.0121 \\
5.70 & 0.0162 & 0.0189 \\
5.63 & 0.0200 & 0.0224 \\
5.20 & 0.0518 & \\
4.81 & 0.100 & \\
4.31 & 0.200 & \\
3.99 & 0.300 & \\
3.56 & 0.500 & \\
2.94 & 1.00 & \\
2.00 & $>2.89$ & \\
1.00 & $>12.8$ & \\
0.50 & $>52$ & \\
\hline
\end{tabular}


Also of interest is a computation with $R=R_{u}, L / R=7, \varepsilon=0, \varepsilon_{R}=0$, and various values of $\left(V / \pi R^{2} L\right)$. It was possible to approach the instabilitystability demarcation from both the stable and unstable regime $[\mathrm{r}(z)=\mathrm{R}$ is a solution in the unstable region for $\left.\left(V / \pi R^{2} L\right)=1\right]$. For $\left(V / \pi R^{2} L\right)=1.270$ and $\left(V / \pi R^{2} L\right)=1.255$ the conjugate points occur at 3.518 and 3.485 , respectively (i.e., the liquid zones are stable and unstable, respectively). Both zones are symmetric about $z=0$; however, the unstable zone has a negative slope at $z=(-L / 2)$ while the stable zone has a positive slope at $z=(-L / 2)$. Thus, the unstable zone has $r_{z}=0$ for three values of $z$ (viz., $z=0$ and $z$ near $\pm L / 2$ ) whereas for the stable zone $r_{z}=0$ only at $z=0$. If we require that the relative change in $s$ be less than $10^{-3}$, the iteration procedure fails for $\left(V / \pi R^{2} L\right)$ equal to 1.260 and 1.265 ; it would work if we slightly relaxed the $10^{-3}$ requirement. The $\left(\mathrm{V} / \pi \mathrm{R}^{2} \mathrm{~L}\right)$ obtained for the stabilityinstability demarcation appears to agree with the results of Gillette and Dyson $^{14}$.

\section{Experimental Observations}

We have made some observations of water zones between non-rotating flat faces of coaxial circular cylinders of equal diameter in order to compare the shape and stability with the predictions of the calculations. By using very small radii, one can simulate microgravity, e.g., for $\mathrm{R}=2.7 \times 10^{-2} \mathrm{~cm}$, $\varepsilon=10^{-2}$. The solid surfaces used have generally been steel, but some measurements have also been made with glass surfaces. The top cylinder is rigidly mounted and the bottom cylinder is clamped to a micromanipulator that permits precise variation of the vertical position. To minimize volume changes during measurement due to evaporation, the steel rods and water zone are enclosed in a glass tube sealed at the top and containing 
a reservoir of water at the bottom.

The theoretical calculations previously discussed have found the maximum stable length $L$ of a zone whose volume is $\pi R^{2} L$, where $R$ is the radius of the end surfaces. The technique used experimentally to reproduce the conditions assumed in the theory is to find the maximum stable length as a function of zone volume. The stability of the zone of volume $\pi \mathrm{R}^{2} \mathrm{~L}$ can then be determined from this function.

The experiments are performed by injecting a small drop of known volume into the space between the two coaxial rods using a micrometer syringe. The liquid zone is then extended by slowly lowering the bottom rod. When the zone fails, the length is recorded, and the process repeated with a different volume. The zone is observed through the microscope during the extension to verify that failure is due to necking of the zone rather than detachment from the upper surface. In all cases the zone wetted the upper face up to the edge.

The result of a typical measurement using this process is presented in Figure 5 which shows the variation of maximum length with zone volume for an end face of radius $0.1205 \mathrm{~cm}$. The line through the origin gives the volume of a cylindrical zone of length $\mathrm{L}$ and radius $0.1205 \mathrm{~cm}$. Thus, the intersection of this line with the experimental curve determines the length at which a cylindrical zone of volume $=\pi \mathrm{R}^{2} \mathrm{~L}$ will fail. For Figure 5 , this intersection is at $\mathrm{V}=2.45 \times 10^{-2} \mathrm{~cm}^{3}$ and $\mathrm{L}=0.515 \mathrm{~cm}$.

A series of measurements of maximum stable length $L$ for a zone of volume $\pi \mathrm{R}^{2} \mathrm{~L}$ have been made using rods of different $\mathrm{R}$. The results are shown in Figure 6 where $(L / R)$ exp is plotted as a function of the Bond number, $\varepsilon=\frac{\rho \mathrm{gR}^{2}}{\gamma}$. Figure 6 also shows the theoretical prediction of the numerical calculations summarized in Table 2 and the dependence suggested by Carruthers 
and Grasso, i.e., $L / R=2 \pi /(1+\varepsilon)^{\frac{1}{2}}$ (the range of $\varepsilon$ in their experiments was approximately 0.4 to 14$)$. Our experimentally measured (L/R) are within about $2 \%$ of the theoretical prediction for all values of $\varepsilon$. An observed zone is compared with a calculated profile in Figure 7 . The agreement is excellent.

There is a large difference between our numerical and experimental results and those of Carruthers and Grasso, e.g., for $\varepsilon=1.35$, they find an $\mathrm{L} / \mathrm{R}$ that is about $55 \%$ larger than our theoretical prediction and our experimentally observed value. The reasons for such large differences are unclear. Perhaps the boundary conditions used in our experimental and theoretical work, viz., $r(-L / 2)=r(L / 2)=R$ and $V=\pi R^{2} L$, do not model their experimental situation. We have done a few calculations with volumes slightly different than $\pi R^{2} L$; these have only a slight effect on the maximum stable length. For example, for $\varepsilon=0.5$, the maximum stable $L / R$ is 3.51 , 3.56 and 3.60 for $\left(V / \pi R^{2} L\right)$ equal to $0.95,1.0$ and 1.05 , respectively. We plan further investigations of the effect of the boundary conditions on the stability criterion. In addition, it is desirable to study the effect of various containing fluids on the zone stability, e.g., are there differences between a vapor and a liquid as containing fluid?

In addition to the measurements of water zones, observations of molten zones in thin tin cylinders have also been made. This work was initiated to simulate an experiment proposed for the sounding rocket program.

We thank H. W. McLaughlin and R. F. Sekerka for many stimulating discussions. 
1. W. G. Pfann, Zone Melting (John Wiley \& Sons, Inc., New York, 1966).

2. W. Keller, Third American Conference on Crystal Growth, Stanford University, 1975, p. 67.

3. W. Heywang, Z. Naturforschg. 11a, 238 (1956).

4. P. I. Antonov, in: Growth of Crystals, Vol. 6a, Ed. N. N. Sheftal (Consultants Bureau, New York, 1968) p. 145.

5. W. Bardsley, F. C. Frank, G. W. Green, and D. T. J. Hurle, J. Crystal Growth 23, 341 (1974).

6. T. Surek and B. Chalmers, J. Crystal Growth 29, 1 (1975).

7. J. R. Carruthers and M. Grasso, J. Crystal Growth 13/14, 611 (1972).

8. J. R. Carruthers and M. Grasso, J. App1. Phys. 43, 436 (1972).

9. 0. Bolza, Lectures on the Calculus of Variations (Dover Publications, Inc., New York, 1961).

10. S. C. Hardy and S. R. Coriell, NBS Space Processing Research (NBSIR 74-611) November 1974, p. 60.

11. J. W. Gibbs, The Scientific Papers of J. Willard Gibbs, Vol. I (Dover Publications, New York, 1961) p. 326.

12. C. W. Gear, Numerical Initial Value Problems in Ordinary Differential Equations (Prentice-Hall, Inc., Englewood Cliffs, N.J., 1971).

13. J. F. Padday, Phil. Trans. Roy. Soc. London A269, 265 (1971).

14. R. D. Gillette and D. C. Dyson, Chemical Engineering 2, 44 (1971). 



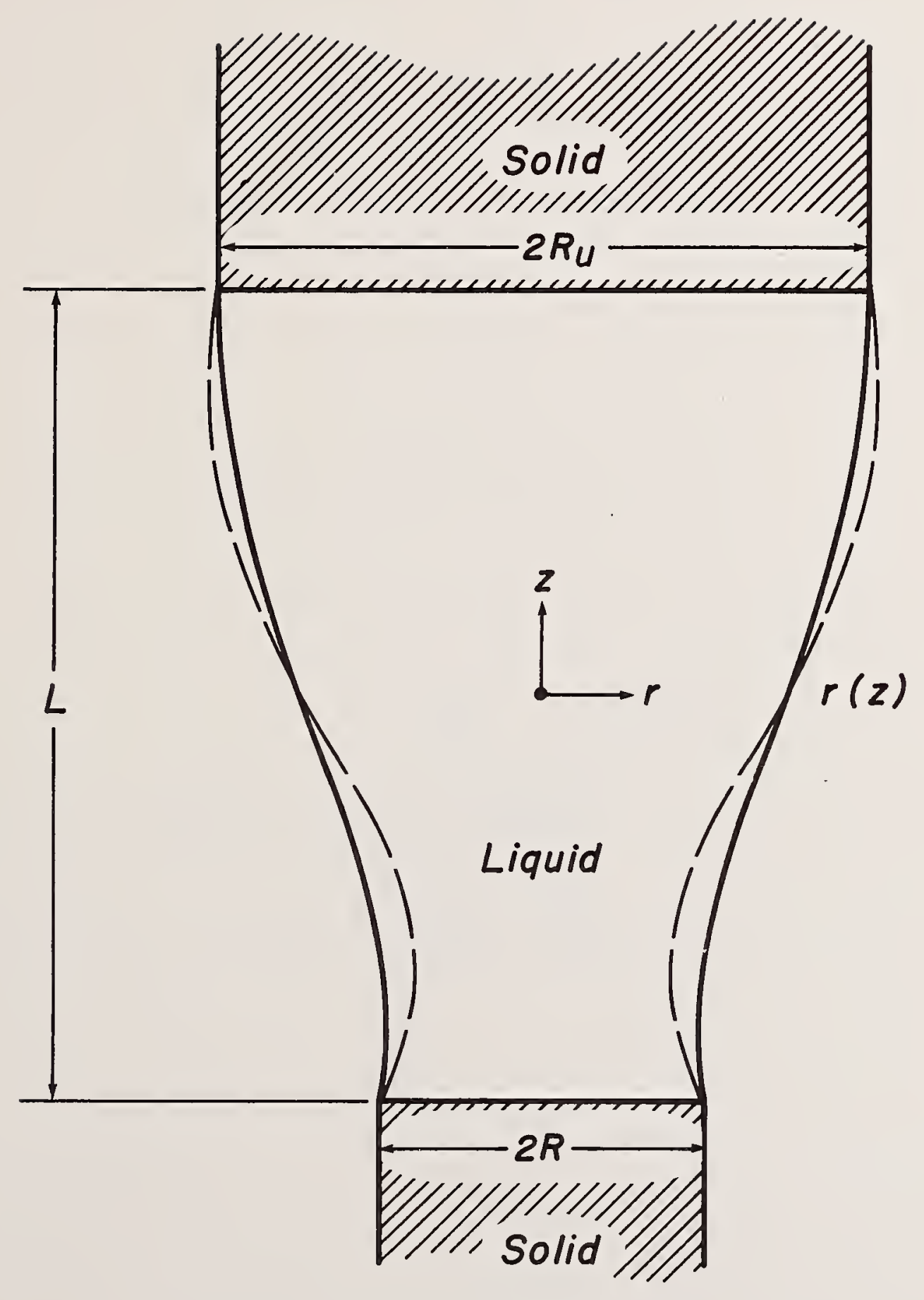

Fig. 1 Stable liquid zones between solids of radil $R_{U}$ and $R$. For these zones $(L / R)=5,2 R=R_{u}$, the volume $V=(7 / 3) \pi R^{2} L$, and the gravitational field is zero. The solid curve corresponds to zero rotation while the dashed curve corresponds to finite rotation $\left(\varepsilon_{R}=0.123\right)$; a $20 \%$ increase in this rotation rate would make the zone unstable. 



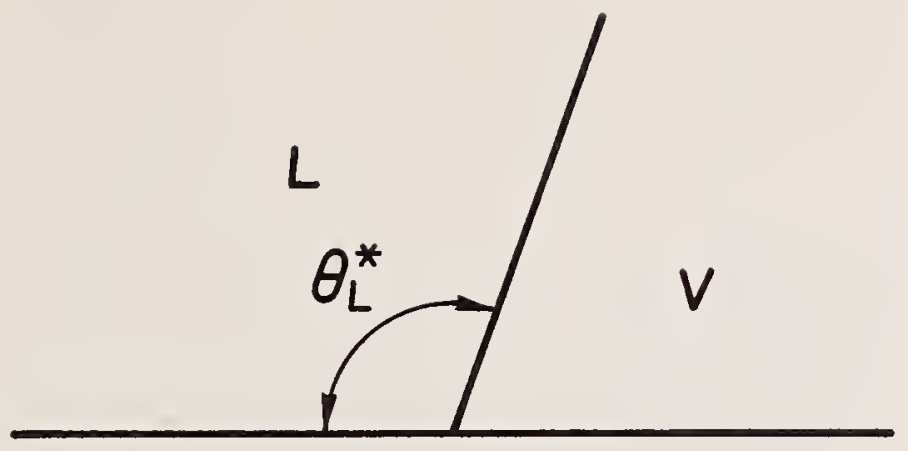

$S$

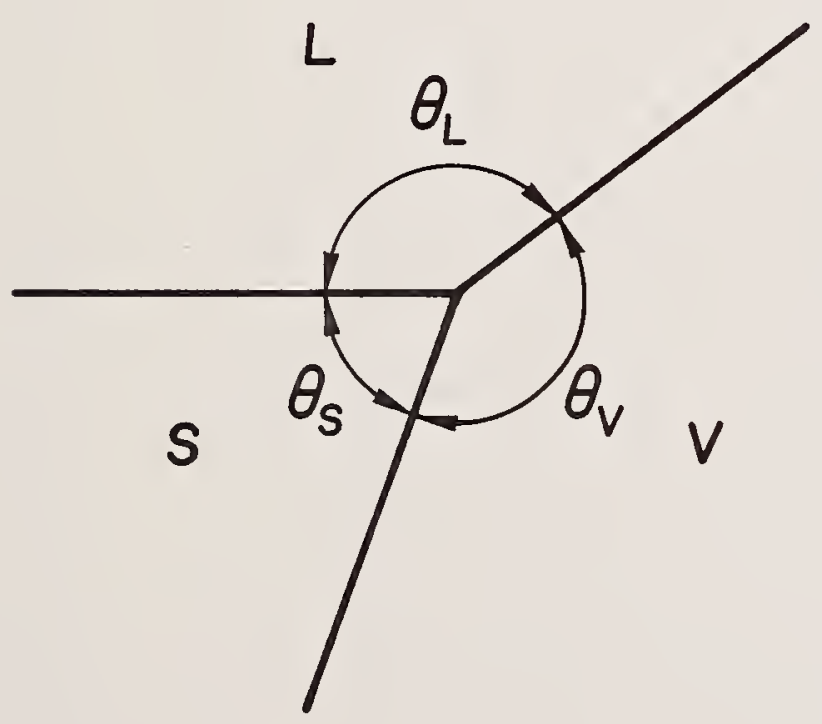

Fig. 2 Trijunctions where solid, liquid and vapor phases meet. In the top figure, the solid surface is flat; in the bottom figure the trijunction occurs at a corner of the solid. 



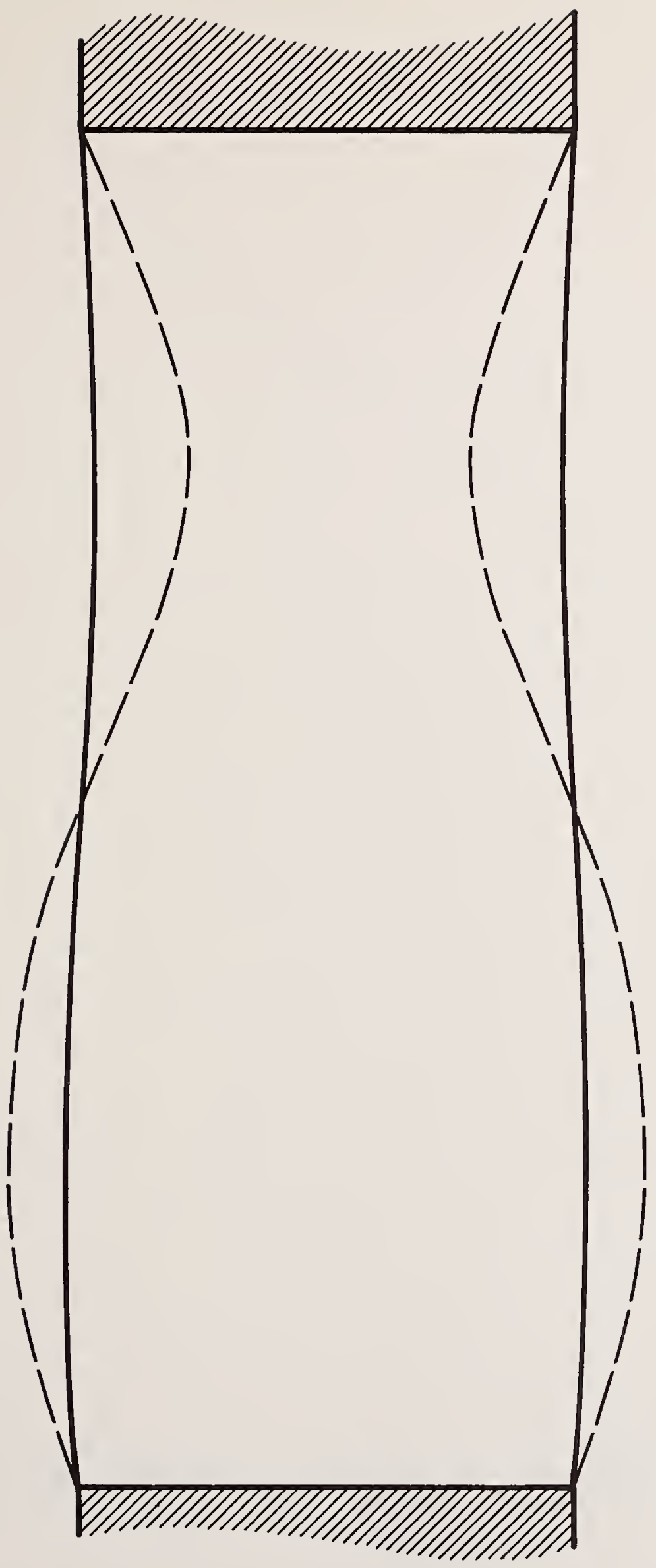

Fig. 3 Two liquid zones satisfying the same boundary conditions and having the same volume. For these zones, $(L / R)=5.5, R=R_{u}, V=\pi R^{2} L, \varepsilon_{R}=0$, and $\varepsilon=0.01$. The zone indicated by the solid curve is stable (the conjugate point $z^{*}=3.512 \mathrm{R}$ ) ; the zone indicated by the dashed curve is unstable $\left(z^{*}=2.020 \mathrm{R}\right)$. Both zone shapes satisfy the Euler equation (with different values of $\mathrm{p}$ ). 



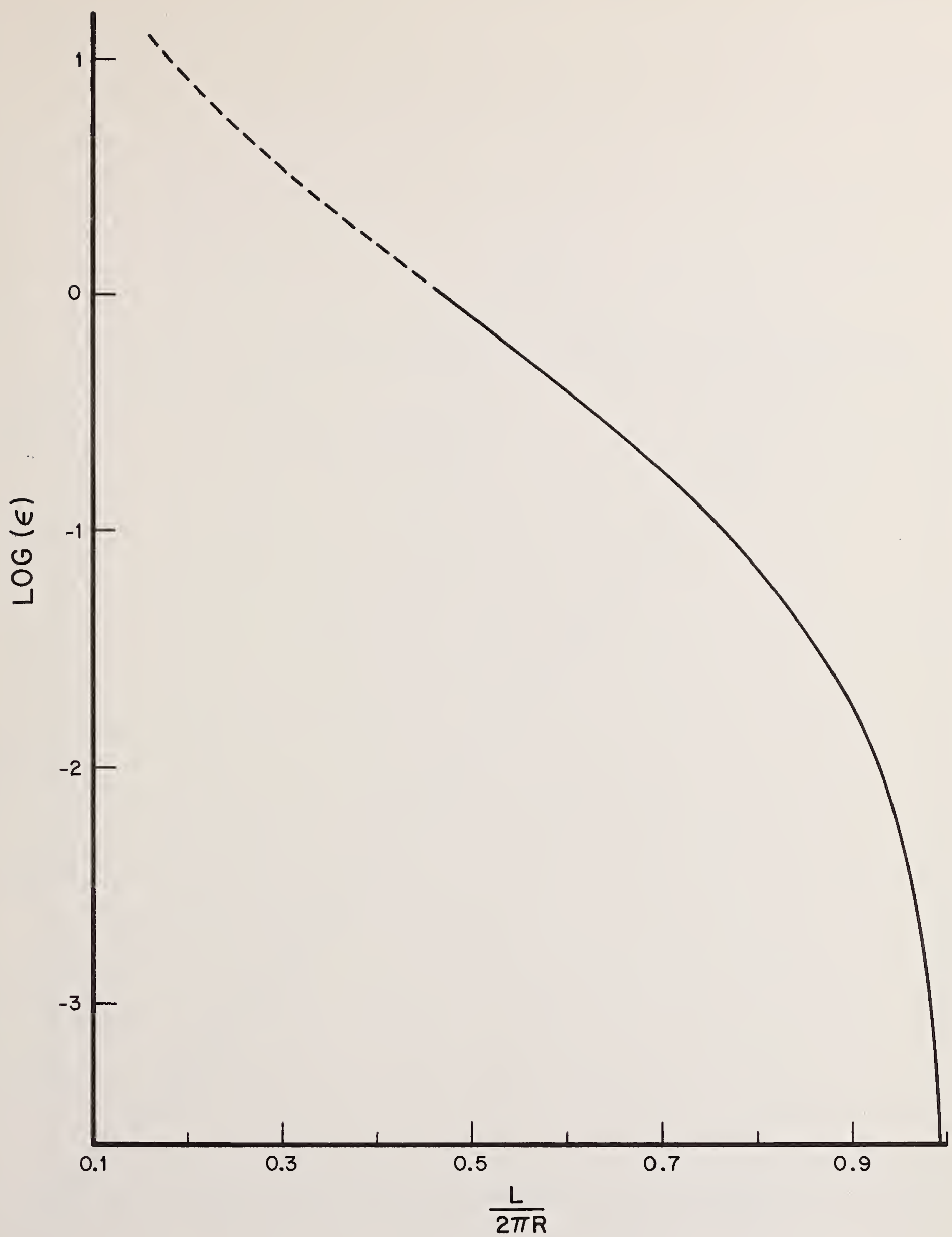

Fig. 4 Numerical results for the stability-instability demarcation; $10 \log _{10} \varepsilon$ as a function of $(\mathrm{L} / 2 \pi \mathrm{R})$ for $\mathrm{R}=\mathrm{R}_{\mathrm{u}}, \mathrm{V}=\pi \mathrm{R}^{2} \mathrm{~L}$, and no rotation. The liquid zones are stable to the left of the curve. The Bond number $\varepsilon \equiv \rho \mathrm{gR}^{2} / \gamma$. 



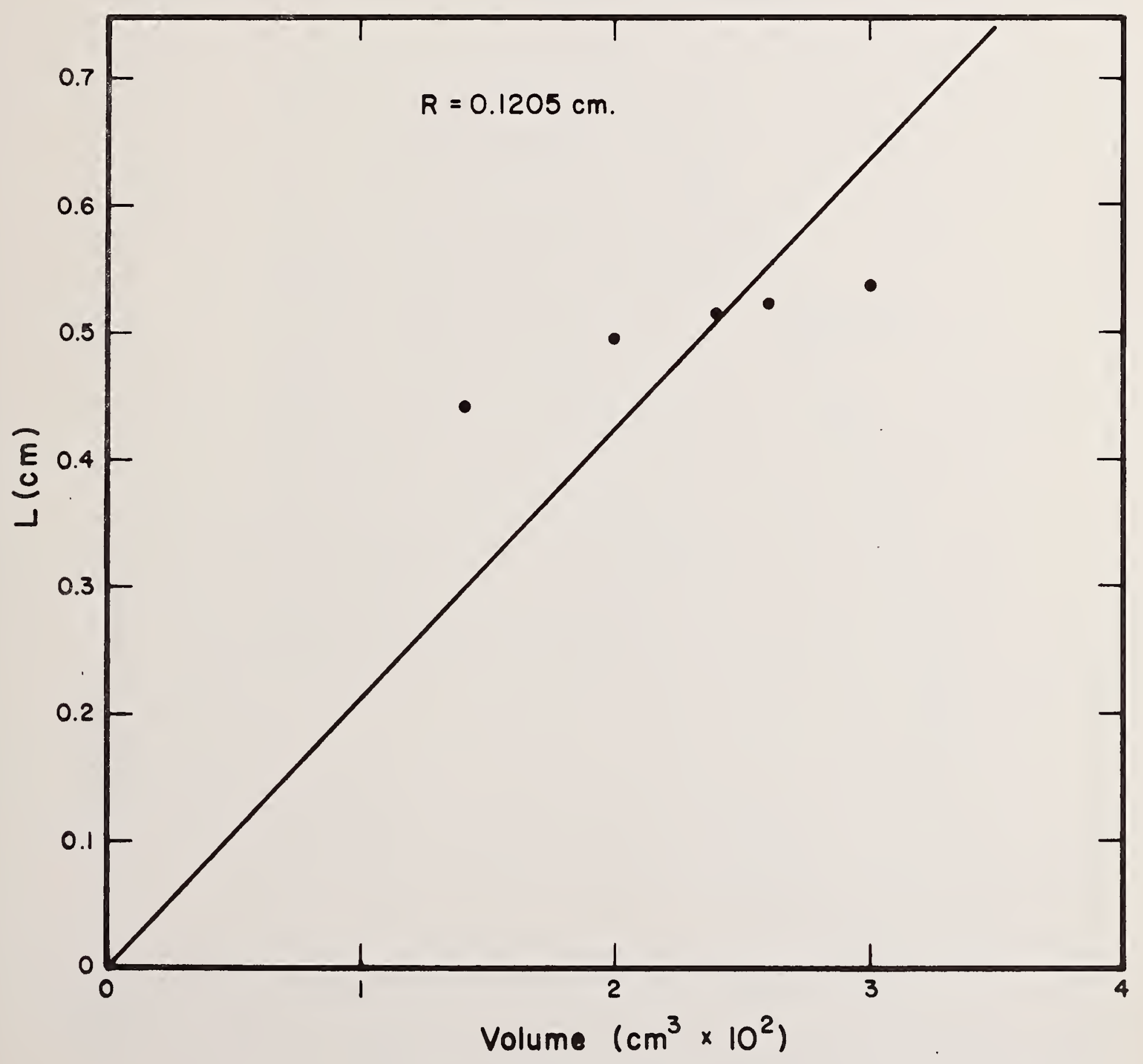

Fig. 5 The maximum stable zone length as a function of zone volume. The straight line indicates the volume of a cylindrical zone. 



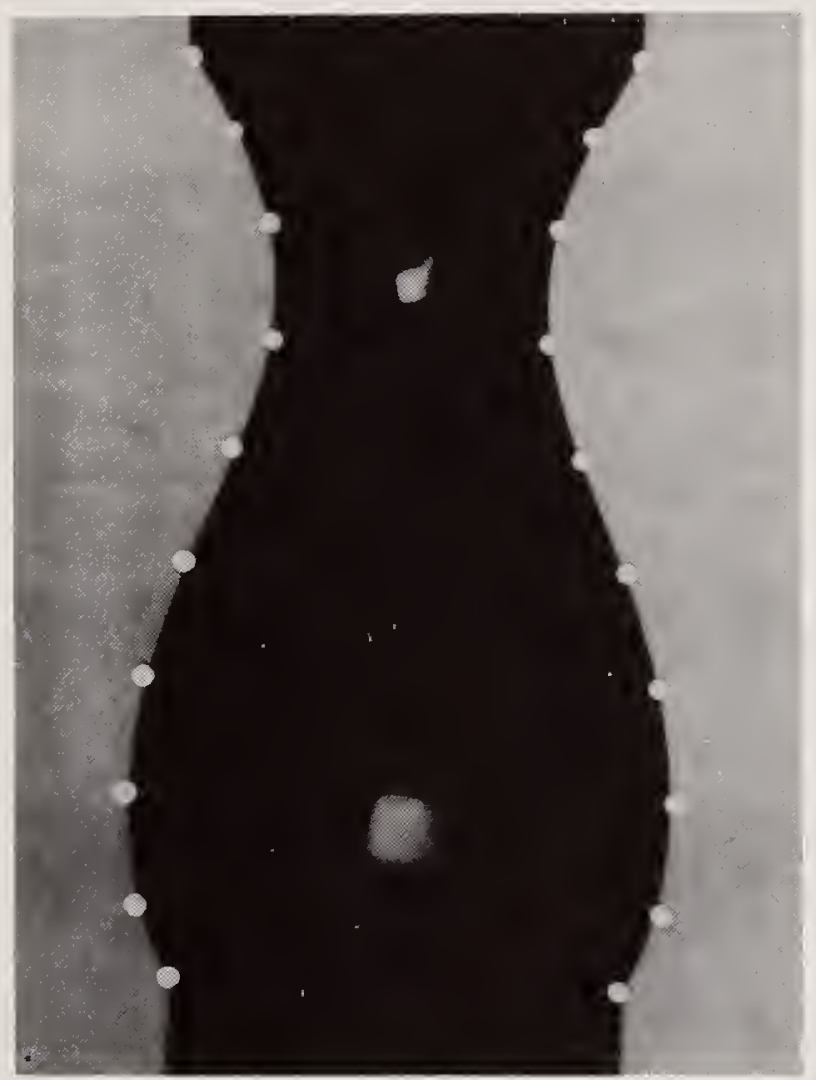

Fig. 7 A comparison of observed and calculated zone shape $\left(\mathrm{V}=.020 \mathrm{~cm}^{3}\right.$, $\mathrm{R}=0.1205 \mathrm{~cm}, \mathrm{~L}=0.497 \mathrm{~cm}, \gamma=72 \mathrm{mJm}^{-2}$, no rotation). 
In this appendix we formulate the relevant differential equations for the shape and stability of a liquid zone when the gravitational field is in an arbitrary direction. For the special case of $R=R_{u}$, the volume $V=$ $\pi R^{2} L$, no rotation, and a small gravitational field, we obtain approximate analytic results for the zone shape and stability; in particular, we find the maximum stable zone length as a function of gravitational field. We also find that a horizontal liquid zone is more stable than a vertical liquid zone.

We apply the calculus of variations ${ }^{9}$ to the stability of a static liquid zone between two solid circular surfaces (plates) which are parallel and coaxial. We assume that the liquid remains at the edges of the plates and that the gravitational field vector makes an angle $\Delta$ with the line joining the center of the circular plates. The distance between the plates is $L$ and the radii of the upper and lower plates are $R_{u}$ and $R$, respectively (subsequently, we take $R_{u}=R$ ). We define a Cartesian coordinate system $(x, y, z)$ with origin at the center of the plates and midway between them. The z-axis coincides with the line joining the center of the plates and is directed toward the upper plate. The $x$-axis is chosen such that the gravitational field vector lies in the $(x-z)$ plane and makes an angle $\Delta$ with the $(-z)$ axis, i.e., $\Delta=0$ corresponds to a gravitational field in the $(-z)$ direction. It is more convenient to use a cylindrical coordinate system $(r, \theta, z)$ which is related to the $(x, y, z)$ system by the usual transformation. The shape of the liquid zone may then be written as $r=r(\theta, z)$ with the boundary conditions $r(\theta,-L / 2)=R$ and $r(\theta, L / 2)=R_{u}$. We also assume that the volume in the zone is fixed, i.e., 
$V=\int_{-L / 2}^{L / 2} d z \int_{0}^{2 \pi} d \theta\left\{r^{2}(\theta, z) / 2\right\}=$ CONSTANT

For a static liquid, the energy is completely determined by the shape of the liquid-vapor interface $r(\theta, z)$. The calculus of variations allows us to determine $r(\theta, z)$ such that the energy is stationary and in addition to determine whether the energy is a minimum and the shape stable. The energy can be written as a sum of surface and gravitational energies, i.e.,

$$
E^{\prime}=\int_{-L / 2}^{L / 2} d z \int_{0}^{2 \pi} d \theta \quad F\left(r, r_{\theta}, r_{z}, \theta, z\right)
$$

with

$$
F\left(r, r_{\theta}, r z, \theta, z\right)=\gamma\left[r^{2}+r_{\theta}^{2}+r^{2} r_{z}^{2}\right]^{\frac{1}{2}}+p g\left[\left(r^{3} / 3\right) \cos \theta \sin \Delta+\left(r^{2} z / 2\right) \cos \Delta\right] .
$$

Here, $\gamma$ is the liquid-vapor surface tension, $\rho$ is the density difference between liquid and vapor, $g$ is the magnitude of the gravitational acceleration, and the subscripts $\theta$ and $z$ denote partial differentiation, e.g., $r_{\theta}=(\partial r / \partial \theta)$ and $r_{z}=(\partial r / \partial z)$. Since the volume is fixed we introduce the Lagrange multiplier $p$ and define $H\left(r, r_{\theta}, r_{z}, \theta, z\right)=F\left(r, r_{\theta}, r_{z}, \theta, z\right)-p r^{2} / 2$ and $E=\int_{-L / 2}^{L / 2} d z \int_{0}^{2 \pi} d \theta\left\{H\left(r, r_{\theta}, r_{z}, \theta, z\right)\right.$.

If $E^{\prime}$ is a minimum, then $E$ is also a minimum since the integral of $r^{2}$ is a constant. In order for $\mathrm{E}$ to be stationary, it is necessary that

$$
(\partial H / \partial r)-(\partial / \partial \theta)\left(\partial H / \partial r_{\theta}\right)-(\partial / \partial z)\left(\partial H / \partial r_{z}\right)=0 .
$$


Upon evaluation of this expression, we obtain the Euler equation

$$
\begin{aligned}
& P \Psi-\varepsilon\left[\Psi^{2} \cos \theta \sin \Delta+\Psi n \cos \Delta\right]=\left\{\Psi^{2}+\Psi_{\theta}^{2}+\psi^{2} \Psi_{\eta}^{2}\right\}^{-3 / 2}
\end{aligned}
$$

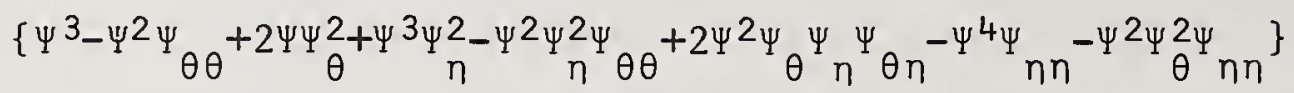

where we have introduced the dimensionless variables, $P=R p / \gamma, \eta=z / R$, $\Psi=r / R, \varepsilon=\rho g R^{2} / \gamma$ and the subscripts indicate partial derivatives. The expression on the right hand side of the Euler equation is equal to $\Psi \mathrm{K}$, where $\mathrm{K}$ is the mean curvature of the surface $\Psi=\Psi(\theta, n)$. The boundary conditions on $\Psi$ are $\Psi(\theta,-\beta)=1$ and $\Psi(\theta, \beta)=\mathrm{R}_{\mathrm{u}} / \mathrm{R}$ with $\beta=\mathrm{L} / 2 \mathrm{R}$. The dimensionless Lagrange multiplier $\mathrm{P}$ is chosen to give the desired volume in the zone.

Using standard techniques from the calculus of variations one calculates the second variation of E. Considering small variations in $\Psi$, i.e., $\psi \rightarrow \psi+\delta \Psi \equiv \psi+W(\theta, n)$, one obtains the Jacobi equation

$$
(\partial J / \partial W)-(\partial / \partial \theta)\left(\partial J / \partial W_{\theta}\right)-(\partial / \partial \eta)\left(\partial J / \partial W_{\eta}\right)=0
$$

with

$$
\begin{aligned}
J= & H_{\Psi \Psi} W^{2}+H_{\Psi_{\eta} \Psi_{\eta}} W_{\eta}^{2}+H_{\Psi_{\theta} \Psi_{\theta}} W_{\theta}^{2}+2 H_{\Psi \Psi} W W_{\theta} \\
& +2 H_{\Psi \Psi} W_{\eta}+2 H_{\Psi_{\eta} \Psi_{\theta}} W_{\theta} W_{\eta}+M \Psi W
\end{aligned}
$$

where the subscripts denote partial derivatives and we have introduced the Lagrange multiplier $M$ in order that the integral

$$
\int_{-\beta}^{\beta} d \eta \int_{0}^{2 \pi} d \theta \Psi W=0,
$$

which ensures that the volume in the liquid zone is constant. The Jacobi equation may be written as

$$
\begin{aligned}
& W\left\{2 \varepsilon \Psi \cos \theta \sin \Delta+\varepsilon n \cos \Delta-P+B \Psi_{\theta}^{2}\left(1+\Psi_{n}^{2}\right)\right\} \\
& -W_{\theta}\left\{B\left[\Psi \Psi_{\theta}+\Psi \Psi_{\theta} \Psi_{n}^{2}\right]\right\}+W_{n}\left\{\left[\left[\Psi^{3} \Psi_{n}\left(1+\Psi_{n}^{2}\right)+2 \Psi \Psi_{n}{ }_{\theta}^{2}\right]\right\}\right.
\end{aligned}
$$




$$
\begin{aligned}
& +\{\partial / \partial \theta\}\left\{B\left[W\left(\Psi \Psi_{\theta}+\Psi \Psi_{\theta} \Psi_{\eta}^{2}\right)+W_{\eta}\left(\Psi^{2} \Psi_{\eta} \Psi_{\theta}\right)-W_{\theta}\left(\Psi^{2}+\Psi_{\eta}^{2} \Psi_{\eta}^{2}\right)\right]\right\} \\
& \text { - }\{\partial / \partial \eta\}\left\{B\left[W\left(\Psi^{3} \Psi_{\eta}+\Psi^{3} \Psi_{\eta}^{3}+2 \Psi \Psi_{\eta} \Psi_{\theta}^{2}\right)+W_{\eta}\left(\Psi^{4}+\Psi^{2} \Psi_{\theta}^{2}\right)-W_{\theta}\left(\Psi^{2} \Psi_{\theta} \Psi_{\eta}\right)\right]\right\} \\
& =-\mathrm{M} \Psi \text {, }
\end{aligned}
$$

where $B=\left\{\Psi^{2}+\psi_{\theta}^{2}+\psi^{2} \Psi_{n}^{2}\right\}^{-3 / 2}$

The stability of the liquid zone is determined by the properties of the Jacobi equation. Let $u(\theta, n)$ and $v(\theta, n)$ be solutions of the homogeneous $(M=0)$ and inhomogeneous (Mf0) Jacobi equation, respectively, such that $u(\theta,-\beta)=$ $v(\theta,-\beta)=0$. Further, let

$$
\begin{aligned}
& \mathrm{m}=\int_{0}^{2 \pi} d \theta \int_{-\beta}^{n(\theta)} \operatorname{dn}\left\{\begin{array}{ll}
\Psi & u
\end{array}\right\} \\
& \mathrm{n}=\int_{0}^{2 \pi} d \theta \int_{-\beta}^{n(\theta)} \mathrm{dn}\{\Psi \mathrm{v}\} \\
& \mathrm{D}[\mathrm{n}(\theta),-\beta]=\mathrm{m} v-\mathrm{n} u
\end{aligned}
$$

The liquid is unstable if in the region $0<\theta \leq 2 \pi$ and $-\beta<n \leq \beta$, there exists a curve (or boundary) $\eta^{*}=\eta^{*}(\theta)$ such that $D\left[\eta^{*}(\theta),-\beta\right]=0$. If no such $\eta *$ exists, the liquid zone is stable.

The preceding paragraphs give the basic equations for the shape and stability of a liquid zone. In principle these equations can be solved numerically for arbitrary volumes, gravitational fields, and upper plate radius $R_{u}$. We proceed however to obtain an analytic solution for the special case where $V=\pi R^{2} L$, the gravitational field is small $(\varepsilon<<1)$ and $R_{u}=R$. We expand all quantities in a power series in $\varepsilon$, and retain terms of the order of $\varepsilon^{2}$, e.g.,

$$
\begin{aligned}
& \Psi=\Psi_{0}+\varepsilon \Psi_{1}+\varepsilon^{2} \Psi_{2} \\
& P=P_{0}+\varepsilon P_{1}+\varepsilon^{2} P_{2}
\end{aligned}
$$




$$
W=W_{0}+\varepsilon W_{1}+\varepsilon^{2} W_{2} \text {, etc. }
$$

In view of the quantitative disagreement between our numerical and analytic results for $\Delta=0$ or $\pi$ (see Tables 1 and 2), this expansion procedure is apparently not rigorous even if $\varepsilon<<1$; the results obtained should be regarded as approximations.

For $\Psi(\theta, \eta)$, we obtain

$$
\begin{aligned}
\Psi_{0} & =1 \\
\Lambda \Psi_{1} & =-P_{1}+\cos \theta \sin \Delta+\eta \cos \Delta \\
\Lambda \Psi_{2} & =-P_{2}+\Psi_{1} \cos \theta \sin \Delta+2 \Psi_{1} \Psi_{1 \theta \theta}+\Psi_{1}^{2}+\frac{1}{2} \Psi_{1}^{2}-\frac{1}{2} \Psi_{1}^{2} n
\end{aligned}
$$

with $\mathrm{P}_{0}=1$ and the operator $\Lambda=1+\left(\partial^{2} / \partial \theta^{2}\right)+\left(\partial^{2} / \partial \eta^{2}\right)$. The boundary conditions on $\Psi_{1}$ and $\Psi_{2}$ are that $\Psi_{1}(\theta, \pm \beta)=\Psi_{2}(\theta, \pm \beta)=0$. The constants $\mathrm{P}_{1}$ and $\mathrm{P}_{2}$ are chosen so that the integrals

$$
\int_{-\beta}^{\beta} d \eta \int_{0}^{2 \pi} d \theta\left\{\Psi_{1}\right\}=\int_{-\beta}^{\beta} d \eta \int_{0}^{2 \pi} d \theta\left\{\Psi_{1}^{2}+2 \Psi_{2}\right\}=0 .
$$

We find that

$\Psi_{1}=\cos \Delta\{\eta-[\beta \sin \eta / \sin \beta]\}+\sin \Delta \cos \theta\left(\eta^{2}-\beta^{2}\right) / 2$ with $P_{1}=0$ and

$$
\begin{aligned}
\Psi_{2}= & \cos ^{2} \Delta\left\{\eta^{2}-\left(\beta^{2} / 2\right)-\left(\beta^{2} / 2\right)\left(\sin ^{2} \eta / \sin ^{2} \beta\right)\right. \\
& \left.+[\beta / \sin \beta]\left[\left(\eta^{2} / 2\right) \cos \eta-\left(\beta^{2} / 2\right) \cos \eta+2 \cos \eta-2 \cos \beta\right]\right\} \\
& +\sin \Delta \cos \Delta \cos \theta\{\beta / \sin \beta\}\{3 \sin \eta-3(\eta / \beta) \sin \beta-\eta \cos \eta+\eta \cos \beta\} \\
+ & \sin ^{2} \Delta\left\{-\left(\eta^{2}-\beta^{2}\right)^{2} / 16+\left(3 \eta^{2} / 4\right)-\left(3 \beta^{2} / 4\right)\right. \\
& +\left(\beta^{3} / 2\right)[\cos \beta-\cos \eta] /[\beta \cos \beta-\sin \beta] \\
& +\cos 2 \theta\left[\left(\eta^{2}-\beta^{2}\right) / 16+\left(\eta^{2} / 4\right)+(1 / 6)\right. \\
& \left.\left.\quad-\left(\cosh \left\{3^{\frac{1}{2}} \eta\right\} / \cosh \left\{3^{\frac{1}{2}} \beta\right\}\right)\left(\left\{\beta^{2} / 4\right\}+\{1 / 6\}\right)\right]\right\}
\end{aligned}
$$

with

$$
\hat{\mathrm{P}}_{2}=\cos ^{2} \Delta\left\{\left(\beta^{2} / 2\right)-(5 / 2)+(2 \beta \cos \beta / \sin \beta)+\left[\beta^{2} /\left(2 \sin ^{2} \beta\right)\right]\right\}
$$


Expanding the Jacobi equation in powers of $\varepsilon$, we obtain

$$
\begin{aligned}
& \Lambda \mathrm{W}_{\mathrm{O}}=\mathrm{M}_{\mathrm{O}} \\
& \Lambda \mathrm{W}_{1}=\mathrm{M}_{1}+\mathrm{M}_{0} \Psi_{1}+\mathrm{W}_{0}\left[2 \sin \Delta \cos \theta+\eta \cos \Delta-\mathrm{P}_{1}+\Psi_{1_{\theta \theta}}-\Psi_{1_{\eta n}}\right] \\
& \text { - } \mathrm{W}_{\text {onn }}{ }^{\Psi_{1}}-\mathrm{W}_{\text {on }}{ }^{{ }^{1}{ }_{1}} \\
& \Lambda W_{2}=M_{2}+M_{1} \Psi_{1}+M_{o} \Psi_{2}+W_{o}\left[2 \Psi_{2 \theta \theta}+\Psi_{2}+\Psi_{1} \cos \theta \sin \Delta\right. \\
& \text { - } \left.(3 / 2) \Psi_{1_{\theta}}^{2}-4 \Psi_{1} \Psi_{I_{\theta \theta}}-\Psi_{1}^{2}+\left(\Psi_{I_{\eta}}^{2} / 2\right)\right] \\
& +W_{1}\left[2 \Psi_{1 \theta \theta}+\Psi_{1}+\cos \theta \sin \Delta\right] \\
& +\mathrm{W}_{o \eta}\left[2 \Psi_{1_{\theta}} \Psi_{1_{\theta \eta}}+\Psi_{1_{\eta}} \Psi_{1_{\theta \theta}}+{ }^{3 \Psi_{1}}{ }_{1_{\eta}} \Psi_{1_{\eta n}}-\Psi_{2_{\eta}}\right] \\
& +W_{o n n}\left[-\Psi_{2}+\left(\Psi_{1_{\theta}}^{2} / 2\right)+(3 / 2) \Psi_{1_{\eta}}^{2}\right] \\
& +W_{1_{\theta \theta}} \Psi_{1}+W_{1_{\theta}}{ }_{1_{\theta}}-W_{1_{\eta}}{ }^{\Psi_{1}}-W_{1_{\eta \eta}}{ }^{\Psi_{1}}
\end{aligned}
$$

In obtaining the equations for $W_{1}$ and $W_{2}$, we have used the result that $W_{0}$ is independent of $\theta$. In fact, the solutions $u_{0}$ and $v_{0}$ of the homogeneous and inhomogeneous Jacobi equations, respectively, are

$$
\dot{u}_{0}=\sin (n+\beta)
$$

and

$$
v_{0}=M_{0}[1-\cos (n+\beta)]
$$

To zeroth order in $\varepsilon$ a simple calculation gives $\eta *(\theta)=2 \pi-\beta$ and the stability criterion is $n^{*}>\beta$, i.e., the well known result $\beta<\pi(L<2 \pi R)$. It is convenient to evaluate quantities at $n_{0}^{*}=2 \pi-\beta$ rather than at $n^{*}=$ $\eta_{0}^{*}+\varepsilon \eta_{1} *+\varepsilon^{2} \eta_{2} *$. Thus, we expand in a Taylor series about $\eta_{0}{ }^{*}$. For example, given a function $f(\theta, \eta)=f_{0}+\varepsilon f_{1}+\varepsilon^{2} f_{2}$, we evaluate this function at $n=n *(\theta)$ by

$$
\begin{aligned}
\mathrm{f}\left(\theta, n^{*}\right)= & \mathrm{f}_{\mathrm{o}}+\varepsilon\left[\eta_{1} * \mathrm{f}_{o n}+\mathrm{f}_{1}\right]+\varepsilon^{2}\left[\left(\mathrm{f}_{o n n} / 2\right)\left(n_{1}^{*}\right)^{2}+n_{2} * \mathrm{f}_{o n}\right. \\
& \left.+\mathrm{f}_{2}+\eta_{1} * \mathrm{f}_{1_{n}}\right],
\end{aligned}
$$


where $f$ and its derivatives on the right hand side of the equation are evaluated at $n_{0}{ }^{*}$. Applying the above formula to the quantities appearing in $D[n(\theta),-\beta]$, we obtain the following expressions for $\eta_{1} *$ and $\eta_{2} *$ :

$$
\begin{aligned}
\eta_{1}^{*}= & -u_{1}\left(\eta_{0}^{*}\right) \\
n_{2} * & -u_{2}\left(\eta_{0}^{*}\right)-\eta_{1}^{*} u_{1_{\eta}}\left(\eta_{0}^{*}\right) \\
& +\left\{v_{1}\left(\eta_{0}^{*}\right) /\left(4 \pi^{2} M_{0}\right)\right\} \int_{0}^{2 \pi} d \theta \int_{-\beta}^{\eta_{0}^{*}} d \eta\left\{u_{0} \Psi_{1}+u_{1}\right\}
\end{aligned}
$$

Since $v_{1}\left(\eta_{0}^{*}\right)$ is proportional to $M_{0}$, the right hand side of the equation for $\eta_{2} *$ is independent of $M_{0}$.

In general $n_{1} *$ and $n_{2} *$ could depend on $\theta$; however, for the particular problem being considered, we will indicate why $\eta_{1} *$ and $\eta_{2} *$ are independent of $\theta$. Specifically we consider $n_{1}^{*}=-u_{1}\left(\eta_{0}^{*}\right)$; now $u_{1}\left(n_{0}^{*}\right)$ may be written as

$$
\begin{aligned}
\mathrm{u}_{1}\left(\eta_{0}^{*}\right)= & -\{\cos \Delta\}\{2 \pi(\pi-\beta)\} \\
& +\sum_{n=1}^{\infty}\left\{B_{\operatorname{sn}} \sin (n \theta)+B_{c n} \cos (n \theta)\right\}
\end{aligned}
$$

where the coefficients $B_{s n}$ and $B_{c n}$ are arbitrary constants. The $u_{1}(n)$ which gives rise to the above form of $u_{l}\left(n_{0}^{*}\right)$ satisfies the appropriate differential equation and the boundary condition $\mathrm{u}_{1}(-\beta)=0$. It is clear that the average value of $u_{1}\left(n_{0}^{*}\right)$ over the interval $0<\theta \leq 2 \pi$ is the $\theta$ independent term $\{-[\cos \Delta][2 \pi(\pi-\beta)]\}$. Further unless $B_{s n}=B_{c n}=0$ for all $\left.n, u_{1} i_{0}^{*}\right)$ will be both larger than and smaller than this average value. This will impose a less stringent test of stability than that obtained by setting $B_{s n}=B_{c n}$ $=0$ for all $\mathrm{n}$. Thus, we require that $n_{1} *$ and $n_{2}$ * be independent of $\theta$. Evaluation of the expressions for $\eta_{1}$ and $\eta_{2}$ yields $n_{1} *=\cos \Delta\{2 \pi(\pi-\beta)\}$ 


$$
\begin{aligned}
n_{2} *= & \pi \cos ^{2} \Delta\left\{-9-6 \pi \beta+4 \pi^{2}+4 \beta^{2}-14 \beta(\cos \beta / \sin \beta)-\left[9 \beta^{2} /\left(2 \sin ^{2} \beta\right)\right]\right\} \\
& +\pi \sin ^{2} \Delta\left\{-\left[3 \beta^{2} / 2\right]+\left[\beta^{3} \cos \beta /(\beta \cos \beta-\sin \beta)\right]\right\}
\end{aligned}
$$

The stability criterion $\beta<n^{*}$ can be written

$$
0<(\pi-\beta)(1+\varepsilon \pi \cos \Delta)+\left(\varepsilon^{2} / 2\right) \eta_{2} *
$$

Assuming $0<\pi-\beta \equiv \mu<1$ and expanding the above inequality in powers of $\mu$, we obtain

$$
\begin{aligned}
& \varepsilon^{2}\left\{\cos ^{2} \Delta\left[\left(9 \pi^{3} / 4 \mu^{3}\right)-\left(23 \pi^{2} / 2 \mu^{2}\right)+(55 \pi / 4 \mu)-\left(\pi^{3} / 4 \mu\right)\right]\right. \\
& \left.+\sin ^{2} \Delta\left[\left(\pi^{3} / 4 \mu\right)\right]\right\}<1+\varepsilon \pi(\cos \Delta)
\end{aligned}
$$

For $\Delta=0$ or $\pi$, the stability criterion is

$$
\beta<\pi\left[1-(3 \varepsilon / 2)^{2 / 3}\right]
$$

while for $\Delta= \pm(\pi / 2)$, the stability criterion is

$$
\beta<\pi\left[1-\left(\varepsilon^{2} \pi^{2} / 4\right)\right]
$$

Thus a gravitational field along the z-axis (which connects the centers of the two end plates) is more destabilizing than a gravitational field perpendicular to the z-axis. 


\section{Task 5}

VAPOR TRANSPORT SYNTHESIS AND CRYSTAL GROWTH

H. S. Parker

Inorganic Materials Division

Institute for Materials Research

\section{Summary}

The major objective of this study is the investigation of advanced techniques for vapor transport synthesis and crystal growth and definition of the limitations imposed by convective effects in a temperature gradient, for comparison with results of similar experiments in near zero-gravity conditions. The growth of mercurous chloride by an evaporation-condensation mechanism has been chosen not only for its adaptability to this method but also for the potential technological importance as an infra-red polarizing material. During the previous contract period, transport rates were shown to be high at temperatures in the $400^{\circ} \mathrm{C}$ range and temperature differences $(\Delta \mathrm{T})$ of $60-80^{\circ} \mathrm{C}$.

Seeded growth experiments were undertaken during this contract period and improvements in the transport tube geometry and technique are described. Growth at temperatures in the $350^{\circ}-390^{\circ} \mathrm{C}$ range at lower $\Delta \mathrm{T}$ has resulted in good quality growth of $0.6 \mathrm{~cm}$ diameter boules over $1 \mathrm{~cm}$ in length.

The existence of a tetragonal-monoclinic transition in mercurous chloride was found to occur at about $168 \pm 5 \mathrm{~K}$ as determined by low temperature, $x$-ray powder diffraction. This transition should be investigated further to determine if the monoclinic phase is non-centrosymmetric in view of the potential as an infra-red laser modulator. 
The major objective of this study is the investigation of advanced techniques for vapor transport synthesis and crystal growth and definition of the limitations imposed by convective effects in a temperature gradient, for comparison with results of similar experiments in near zero-gravity conditions. Mass transport in the presence of a temperature gradient can be achieved either by the addition of a suitable transporting agent, generally a halide, or by a simple evaporation-condensation mechanism. The general applications of the technique have been described by Schafer [1], Laudise [2], and more recently by Kaldis [3]. In growth by chemical transport, the reversible reaction occurring at source and growth regions between the material and transporting agent must be considered, and separation of the effect from other growth parameters is often difficult. In those cases where the vapor pressure of the material is sufficiently high, transport and growth can occur via a simpler evaporation-condensation process. However, independent control of the total pressure in the system is lost, since the vapor pressure of the material is fixed solely by the operating temperature. The supersaturation and growth rate are, in part, determined by the choice of $\Delta \mathrm{T}$ and thus $\Delta \mathrm{p}$. In both cases, however, convective and diffusive effects are closely interrelated in a gravity environment. Independent control of parameters such as temperature, temperature gradient and pressure can seldom be achieved without influencing the relative contributions of convection and diffusion to the observed transport. 
The work described in this report is a continuation of the study of the growth of mercurous chloride by an evaporation-condensation mechanism as described in the previous report [4]. Although the growth of mercurous chloride by the vapor Bridgman technique has been demonstrated by previous work at NBS and elsewhere $[5,6,7]$, the present study is primarily concerned with attempts to successfully achieve single crystal growth in a static system. That is, by evaporation-condensation in a temperature gradient with no relative motion of transport tube and gradient.

In addition, the behavior of mercurous chloride at low temperatures was examined by $x$-ray diffraction to determine if a phase transition existed. Such a transition, if to a phase which lacked a center of symmetry, could make mercurous chloride (cooled below the transition temperature) an important material for infra-red laser modulator or phase matching applications. A brief survey of compounds in the $\mathrm{Tl}-\mathrm{Pb}-\mathrm{Cl}-\mathrm{Br}$ systems was made to establish if compounds of technological importance as infra-red materials might exist in these systems and be adaptable to vapor growth techniques. Experimenta1 Procedures

The details of the crystal growth procedure, including a description of the multiple-tapped gradient furnace and ampoule loading procedure was reported previously [4] and only modifications will be described here. During the period of this report, efforts were devoted primarily to seeded growth experiments. All experiments conducted in the tapped gradient furnace were made utilizing a geometry which minimized the convective effect, that is, the transport ampoule was vertical, with the hotter (source) end at the top. A "transparent" furnace with a gold-coated 
quartz tube as thermal insulation was used for some growth runs, permitting continuous observation of charge and seed. This furnace does not have a tapped winding and was operated horizontally to provide a suitable thermal environment.

Seeds of suitable dimensions (approximately $3 \times 3 \times 6 \mathrm{~mm}$ ) were prepared by cleaving from a vapor Bridgman grown boule of good optical quality. Prior to insertion of the ampoule into the transport furnace, a brief thermal etch was given the seed in a separate furnace.

In the initial seeded experiments, the transport tube was inserted into the gradient furnace at cemperature. In subsequent experiments, an auxiliary heater was added to the seed end of the transport tube to maintain a small reverse gradient until the tube equilibrated with the furnace temperature. At that time, power to the auxiliary heater was manually reduced slowly in an attempt to provide a gradual increase in the transport rate.

Because of continuing problems with nucleation on several sites at the seed end of the tube, two major modifications were adopted. Experimental runs were started with transport tube and furnace at room temperature and raised to the desired growth temperature over a six hour period, maintaining the desired final gradient during the heating period. The second modification was to the transport tube. Two concentric quartz tubes are utilized at the seed end of the ampoule, with a 2-3 mm annular space. The inner tube is pointed and sealed at one end and attached to the closed end of the outer cube by a quartz rod of 2-4 inm diameter. The annular space is then evacuated and sealed off prior to charging the tube. 
In this fashion, the heat flow is primarily axial in the growth region, with the tip the coldest part. After an initial period of heating in a reverse temperature gradient, the normal gradient conditions are established and transport proceeds.

In order to obtain $x$-ray diffraction patterns of mercurous chloride at low temperatures, use was made of a commercial powder diffraction unit which uses a Geiger-Muller counter in conjunction with a high angle goniometer having a horizontal axis. A copper casing was designed to surround the conventional glass slide normally used with this equipment. Nitrogen gas, chilled by passing through about 14 meters of copper tubing passing through a liquid nitrogen bath, was led to the copper casing through insulated tubing and directed over the slide and specimen. A rotating seal, coaxial with the goniometer, permitted operation of the goniometer in the usual fashion. Temperatures were monitored by a copper-constantan thermocouple attached to the slide at the edge of the specimen. Temperatures as low as about $95 \mathrm{~K}$ were easily reached. To prevent formation of ice on the specimen surface, the x-ray shield of the goniometer was covered with a thin plastic film which did not seriously attenuate the diffracted beam. Phase relations in the systems $\mathrm{Tl}-\mathrm{Pb}-\mathrm{Cl}-\mathrm{Br}$ were examined by conventional phase equilibrium techniques. Mixtures were prepared from end-member compounds, reacted at various temperatures in evacuated sealed quartz tubes, quenched and examined by petrographic microscope and $\mathrm{x}-\mathrm{ray}$ diffraction methods. 


\section{Experimental Results}

Transport experiments reported previously [4] have shown that at source temperatures in the $405-410^{\circ} \mathrm{C}$ range and a $\Delta \mathrm{T}$ of $60-80^{\circ} \mathrm{C}$, corresponding to a $\Delta \mathrm{p}$ of about 1.5 atmospheres, transport rates in excess of 0.4 grams per hour are obtained. Similar experiments conducted in the same minimum convection geometry but at much lower $\Delta \mathrm{T}$ have shown similar rates. With the source at $411^{\circ} \mathrm{C}$ and a $\Delta \mathrm{T}$ at $15^{\circ}$ (corresponding to a $\Delta p$ of $0.8 \mathrm{~atm})$, a rate of 0.21 grams per hour over an average transport path length of $8 \mathrm{~cm}$ was observed. With the same source temperature and a $\Delta \mathrm{T}$ of $27^{\circ}$ (corresponding to a $\Delta \mathrm{p}$ of $1.0 \mathrm{~atm}$ ), a rate of 0.76 grams per hour was observed. The reason for the inconsistency in the relationship of rate to $\Delta \mathrm{T}$ is not presently known. A possible explanation may be that the actual $\Delta \mathrm{T}$ within the transport tube differs considerably from that shown by the monitoring thermocouples.

All seeded experiments in which the transport tube was inserted with the furnace at temperature were unsuccessful. The initial deposit was polycrystalline in all cases. Use of a small auxiliary heater to provide a small reverse gradient in the initial heating to temperature of the transport tube did not aid in preventing formation of a polycrystalline deposit. The use of the modified transport tube described above yielded much more satisfactory results. After an initial period of heating in a reverse gradient, normal gradient conditions are established and growth proceeds. At a source temperature of $390^{\circ} \mathrm{C}$ and a $\Delta \mathrm{T}$ of $20^{\circ} \mathrm{C}(\Delta \mathrm{p}=0.6$ $\mathrm{atm}$ ) boules $6 \mathrm{~mm}$ diameter and $6-8 \mathrm{~mm}$ in length were obtained in eight hour growth runs. It was found that if the vacuum-insulated portion of 
the tube is too long, it is impossible to maintain a temperature gradient over the entire region available for growth. As a result, growth continues only until the source and growth interfaces are essentially isothermal with the free volume in the tube. The optimum length of the insulated region in the present furnace appears to be about $2 \mathrm{~cm}$.

The most success in seeded growth was obtained by placing the modified transport tube in the furnace at room temperature and raising the furnace temperature to the desired growth temperature over a six hour period. The final $\Delta \mathrm{T}$ was maintained during this heating period. At a source temperature of $355^{\circ} \mathrm{C}$ and $\Delta \mathrm{T}$ of $15^{\circ}(\Delta \mathrm{p}=0.1$ atm) over the last six centimeters of transport distance, transport times of 36 hours were used to provide specimens of reasonable size for evaluation. The total transport distance was 12 centimeters. Optical quality of the resulting boules was much better than previously obtained, and single crystal areas in excess of $1 \mathrm{~cm}$ in length were obtained. See Figure 1.

The use of the horizontal "transparent" furnace for visual monitoring of transport rates and progress of growth is still undergoing development. Attempts at seeded growth runs were unsuccessful, resulting in polycrystalline deposits, apparently due to too large a $\Delta \mathrm{T}$ between source and seed for this furnace geometry. Several growth runs were made with the source at $390^{\circ} \mathrm{C}$ and a $\Delta \mathrm{T}$ as 1 ow as $10^{\circ} \mathrm{C}(\Delta \mathrm{p}=0.2 \mathrm{~atm})$. In future experiments, it is planned to use a small negative gradient (seed hotter) during the heating up cycle. In this case, it will be necessary to use a seed of sufficient size to prevent complete evaporation before the gradient is reversed. 
As previously indicated, if mercurous chloride exhibits a phase transition to a non-centrosymmetric form, it would have the potential for phase-matching and modulator applications over a broad infra-red range. For this reason, it seemed desirable to investigate the possibility of such a transition. An x-ray powder diffraction pattern of mercurous chloride was obtained at $98 \mathrm{~K}$. The pattern was indexed as monoclinic using a least squares calculation yielding unti cell dimensions of $a=c=4.4382 \pm 0.0005 \AA, b=10.905 \pm 0.001 \AA$ and $\beta=90.40^{\circ}$. The room temperature is tetragonal with $a=4.4801 \pm 0.0005 \AA$ and $c=10.907 \pm 0.001 \AA$. In order to determine the transition temperature, the split of the tetragonal 220 reflection into the monoclinic 202 and $20 \overline{2}$ reflections were observed at a series of temperatures between $98 \mathrm{~K}$ and $298 \mathrm{~K}$. These data are shown in Figure 2 and indicate the transition to occur at $168 \pm 5 \mathrm{~K}$. No discontinuous effects were noted for the change of the tetragonal 008 reflection to the monoclinic 080 reflection indicative of no change in the tetragonal c-axis at the transition. A manuscript describing this work in greater detail is in preparation. It is hoped that in the future a definitive study can be made of the lowtemperature phase to determine presence or absence of a center of symmetry. The compounds of major interest in the $\mathrm{T} 1-\mathrm{Pb}-\mathrm{Cl}-\mathrm{Br}$ systems were found to be $\mathrm{Tl}_{3} \mathrm{PbCl}_{5}$ and the corresponding bromide, $\mathrm{Tl}_{3} \mathrm{PbBr}_{5} \cdot$ Based on $x$-ray precession photographs of $\mathrm{Tl}_{3} \mathrm{PbCl}_{5}$, the compound appears to be of tetragona1, non-centrosymmetric symmetry. The bromide was found to be orthorhombic at room temperature, with a transformation to tetragonal 
symmetry at about $280^{\circ} \mathrm{C}$ as determined by elevated temperature $\mathrm{x}$-ray powder diffraction. Considerable solid solubility was found to exist between these compounds. A lowering of the transition temperature of the bromide could be followed with increasing additions of the chloride, up to 20 mole percent chloride and a transition temperature of about $155^{\circ} \mathrm{C}$. Conclusion and Discussion

Vapor transport experiments for the growth of mercurous chloride during the course of the current and previous work of this project have shown that high transport rates can be achieved at temperatures below $400^{\circ} \mathrm{C}$. One of the major problems in the growth of mercurous chloride by an evaporation-condensation in a temperature gradient which is fixed with respect to the growth tube as been nucleation at several sites near. the colder end, even with seeds present. In the usual vapor Bridgman technique where the growth tube is slowly moved through the gradient, the problem is less common, although extremely slow growth rates, of the order of $2 \mathrm{~mm}$ per day, are required for high quality growth. To provide specimen crystals in shorter growth times in order to be compatible with expected durations of low gravity experiments in space, much effort has been devoted to utilizing faster transport rates which means greater supersaturation at the growing interface. On the basis of results obtained thus far, it appears that growth periods of at least 24 - 36 hours will be required for good quality growth under optimum conditions. The use of lower temperature gradients to reduce transport rates and improve crystal quality will also impose a greater 
burden on the temperature control of the furnace. The use of an axial heat sink and vacuum insulation at the growth end of the transport tube shows considerable promise.

On the basis of the results of this study and the technological potential of mercurous chloride as an infra-red material, a proposal was submitted to NASA in response to NASA-AAFE request AO No. OA75-3 suggesting the vapor growth of mercurous chloride by evaporation-condensation as a part of the Advanced Applications Flight Experiment Program.

Further investigation of the low temperature monoclinic form of mercurous chloride should be pursued to determine the presence or absence of a center of symmetry in view of the further potential application of a non-centrosymmetric form as a modulator or phase-matching material for infra-red laser applications. As a further extension of growth by evaporation-condensation, other potentially useful materials such as the thallium-lead halides should be examined for adaptability to this technique. 
[1] a) H. Schafer, "Chemical Transport Reactions", Academic Press, New York, 1964.

b) H. Schafer, "Preparative Methods in Solid State Chemistry", P. Hagenmu1ler, Ed., 251-277, Academic Press, New York, 1972.

[2] R. A. Laudise, "The Growth of Single Crystals", 225-256, PrenticeHa11 Inc., New Jersey, 1970.

[3] E. Kaldis, in "Crystal Growth, Theory and Techniques, Volume I", C.H.L. Goodman, Ed., 49-191, Plenum Press, New York, 1974.

[4] E. Passaglia and R. L. Parker, "NBS Space Processing Research", NBSIR 74-611, 69-78, November 1974.

[5] R. A. Forman, W. S. Brower and H. S. Parker, "Mercurous Chloride Polarizer Material", AFML-TR-74-56, April 1974.

[6] R. A. Forman, W. S. Brower and H. S. Parker, "U.S. Patent 3,902,782, Mercurous Chloride Prism Polarizers", Assigned to United State of America, September 2, 1975.

[7] C. Barta, Kristali und Technik 5 , 541 (1970). 

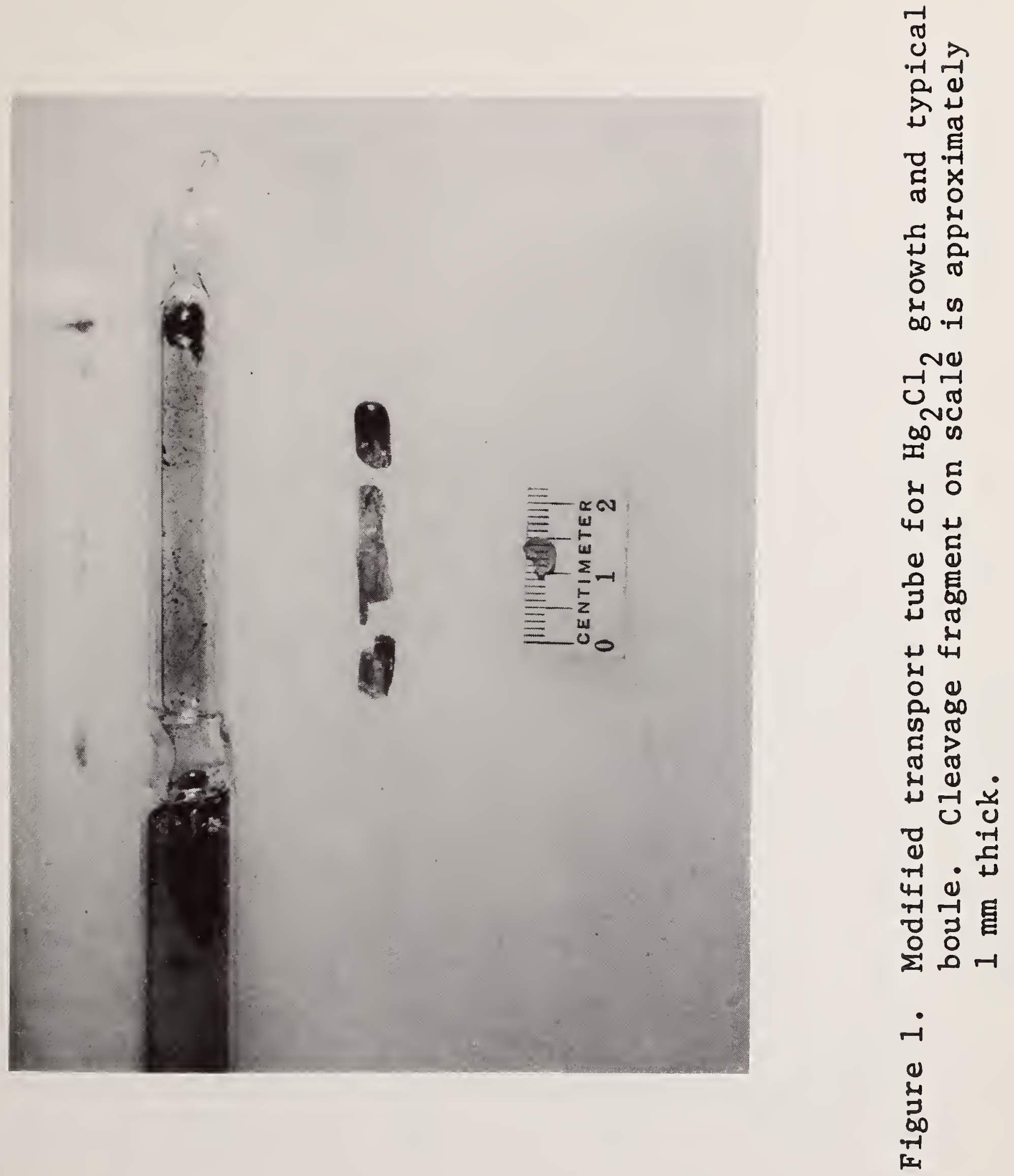



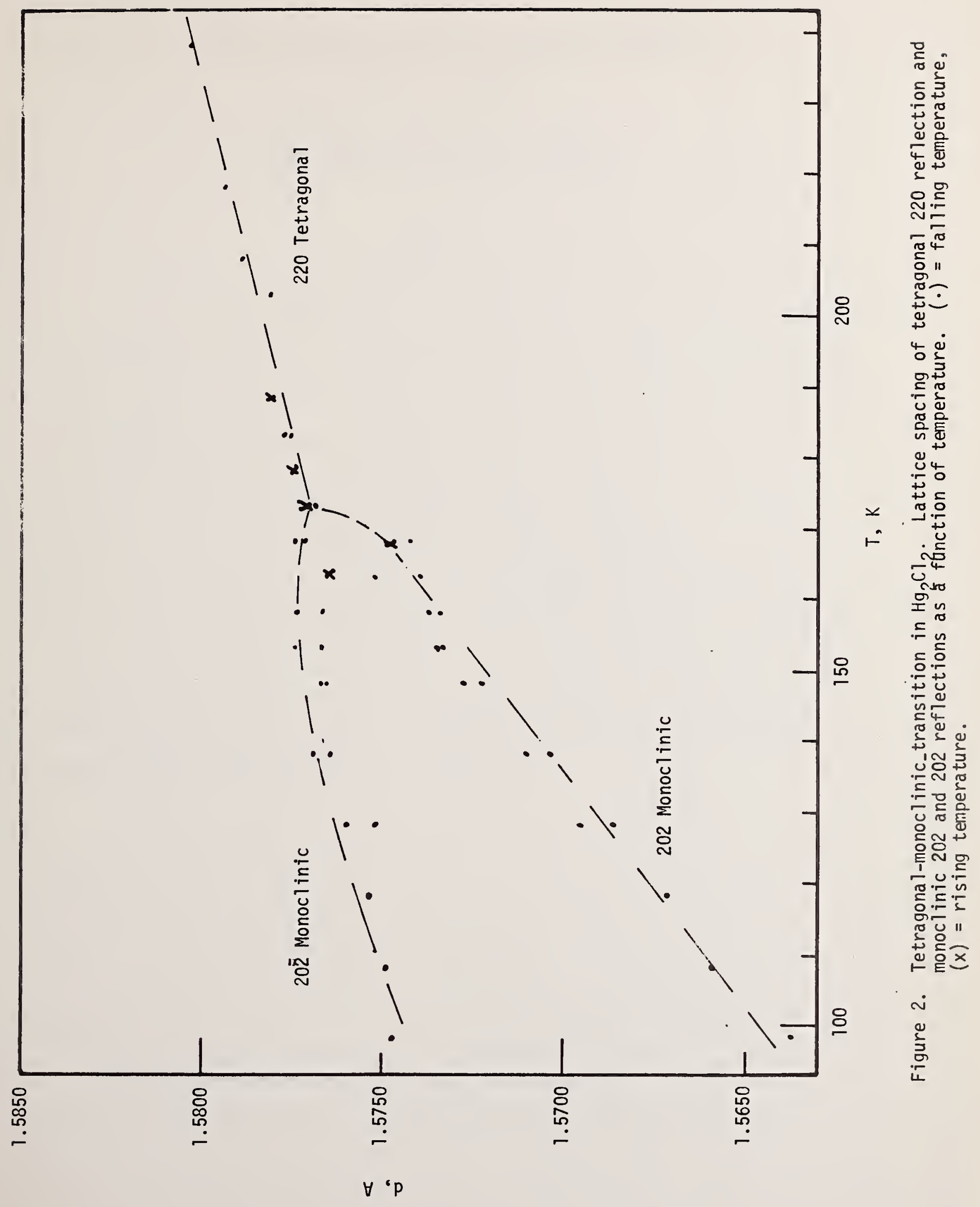



Task 6

Surface Traction and Other Surface Phenomena

\author{
Alan L. Dragoo \\ Inorganic Materials Division \\ Institute for Materials Research
}

\title{
Summary
}

Ground-based experiments and apparatus were developed for studies of convection in drops. A cell consisting of a spherical glass bulb with a heater below and a heat exchanger above to establish a vertical temperature gradient was assembled for studies of convection in a spherical volume of fluid. Preliminary work with this apparatus indicated some of the photographic procedures required in a similar study of thermocapillary convection at low-gravity and provided an estimate of the temperature gradient required for such an experiment using a drop of light fluorocarbon oil. A neutral buoyancy cell was assembled for studies of the coalescence of drops and of possible non-thermal driving-forces for convection. 


\section{Introduction}

Although thermocapillary flow in drops may require low-gravity so that a drop can be levitated for a long period, ground-based studies can be used to develop experimental techniques, to provide a buoyancy analogue upon which thermocapillary flow can be modeled and to estimate conditions for thermocapillary flow. This report is devoted largely to two such ground-based experiments which were initiated in the last year.

The first experiment consists of observations and measurements of flow in a liquid contained in a spherical bulb which is heated from below and cooled from above. The objectives of this experiment are to develop a buoyancy analogue to first-order themocapillary flow in a drop, to develop experimental procedures and to estimate conditions for thermocapillary flow. In conjunction with this experiment, some theoretical work was performed on the problem of buoyancy convection in a fluid sphere with rigid walls. Preliminary experimental results and observations about experimental techniques which are relevant to a study of thermocapillarity will be presented in the report.

The second experiment is intended to study the behavior of silicone oil drops in a neutral buoyancy cell. This cell will be used for studies of the coalescence of drops and to investigate possible non-thermal driving-forces for convection--for example, oscillating electric and acoustic fields. In this report, the neutral buoyancy cell will be described. 
Although buoyancy-driven convection in a bulb of liquid is not exactly comparable to thermocapillary convection in a drop with a free surface, there is sufficient similarity between the two phenomena that buoyancy flow can serve as a model for thermocapillary flow. The two types of flow are approximately similar in two different liquids if

$$
\frac{\text { Buoyancy force in liquid 非1 }}{\text { Viscosity of liquid } \|_{1}} \approx \frac{\text { Surface traction of liquid } \#_{2}}{\text { Viscosity of liquid } \#_{2} \text {. }}
$$

For buoyancy convection, this ratio is the Grashof number, $\mathrm{N}_{\mathrm{Gr}}$. For thermocapillary convection, this ratio is given by the ratio, $\mathrm{Na}_{\mathrm{Ma}} / \mathrm{N}_{\mathrm{Pr}}$, of the Marangoni number to the Prandt1 number. These numbers are expressed in terms of the fluid properties in the table of nomenclature at the end of this chapter of the report. As shown in Table 1 , by choosing an oil with a kinematic viscosity on the order of $100 \mathrm{cs}^{*}$ for the ground-based, buoyancy study, a condition can be achieved which approximates the thermocapillary convection in a drop of Krytox oil**' with a kinematic viscosity of about 35 cs. Using a model similar to the one developed by Chandrasekar [1] for the problem of thermal instability in fluid spheres, the hydrodynamic equations were solved in the linearized Boussinesq approximation. The present model differs from that of Chandrasekar in assuming an applied temperature field with a $\cos \theta$-dependence in contrast to an applied field with only a radial dependence

*The unit "cs" stands for centistokes, $1 \mathrm{cs}=10^{-2} \mathrm{~cm}^{2} / \mathrm{s}=10^{-6} \mathrm{~m} / \mathrm{s}$. **The use of a commercial product name is intended only for the purpose of identification and does not imply approval or acceptance by National Bureau of Standards nor does it imply that the product is necessarily the best material for the purpose described in this report. 
Table 1. Comparison ${ }^{+}$of Liquids for Ground-Based Study (G-B) of a Liquid in a Spherical Bulb and for Low-Gravity Study (L-G) of a Levitated or Tethered Drop

$\begin{array}{lccc}\text { Liquid } & \text { Study } & \underline{v, 10^{-2} \mathrm{~cm}^{2} / \mathrm{s}} & \frac{\mathrm{N}_{\mathrm{Ma}} / \mathrm{N}_{\mathrm{P}}}{143 \mathrm{AA}} \\ \text { fluoro- } & \text { L-G } & 36 & 0.411\end{array}$

Glycero1

G-B

757

0.00659

DC 200 silicone oil

O1ive $0 i 1$

11
11
11

"1

$$
\begin{array}{r}
100 \\
200 \\
500 \\
1000
\end{array}
$$

0.490 .

0.118

0.0188

0.00470

These comparisons are made for a drop or a bulb with a radius of $1 \mathrm{~cm}$ and a constant temperature gradient in vertical direction of $1^{\circ} \mathrm{C} / \mathrm{cm}$.

as in his work. The complete temperature field is specified by

$$
T(r, \theta)=T_{0}-T_{1} r \cos \theta+\theta(r, \theta),
$$

where $\theta(r, \theta)$ is the perturbation associated with the fluid flow. The present model also was solved only for steady flow since only a comparison with previous work [2] on steady thermocapillary flow was desired. By making a further assumption about the solution for $\theta$, single mode solutions can be obtained for the velocity-field problem -that is,

$$
w=r u_{r}=W(r) P_{\ell}(\theta),
$$


where $u_{r}$ is the radial component of the velocity and

$$
W(r)=a_{1} r^{\ell}+a_{3} f_{\ell}(\lambda r)
$$

for the mode of order $\ell$. The function $f_{\ell}\left(\lambda_{r}\right)$ is the spherical Bessel function $j_{\ell}(\lambda r)$ if $\lambda$ is real and the modified spherical Bessel function $i_{\ell}(\lambda r)$ if $\lambda$ is imaginary. The set $\{\lambda\}$ of wavenumbers is defined by the condition $\mathrm{f}_{\ell+1}(\lambda R)=0$. The theta-component of the velocity is

$$
u_{\theta}=-\frac{1}{\ell(\ell+1)}\left[\frac{d w}{d r}+\frac{w}{r}\right] \quad P_{\ell}(\theta)
$$

Consiclering the $\ell=1$ mode,

$$
\begin{aligned}
& u_{r}=\left[a_{1}+a_{3} f_{1}(\lambda r) / r\right] \cos \theta \\
& u_{\theta}=-\left[a_{1}+a_{3} \lambda\left(2 f_{0}(\lambda r)-f_{2}(\lambda r)\right)\right] \sin \theta .
\end{aligned}
$$

Eqns. (5a) and (5b) can be compared to the similar results for steady thermocapillary flow--eqns. (31a) and 31b) in [2]. Moreover, for small $r$, eqns. (5a) and (5b) become approximately

$$
\begin{aligned}
& u_{r} \approx\left[a_{1}+a_{3} \lambda-a_{3} \lambda^{3} r^{2} / 6\right] \cos \theta \\
& u_{\theta} \approx-\left[a_{1}+2 a_{3} \lambda-a_{3} \lambda^{3} r^{2} / 3\right] \sin \theta
\end{aligned}
$$

It is this region of small $\mathrm{r}$ which is most amenable for study by photographic techniques.

Photographic techniques for observing circulation in drops rising or descending in liquid columns were reviewed by Kintner, et. al. [3] In these techniques, flow patterns were traced out by fine particles entrained in the drops. These authors discussed a variety of stilland motion-picture techniques, methods of illumination, methods for time-marking and a procedure for data analysis. 
Particles moving near the center of the drop yield photographs with the least amount of distortion of their image traces due to the spherical lens effect of the drop. The motions of particles in the neighborhood of the drop's center are also the easiest to interpret in terms of the first-order convective mode. For convection driven by a vertical temperature gradient, the flow in the neighborhood of the equatorial plane will be predominately upward or downward across the plane. Such flow is characteristic of all odd-order modes; even-order modes give flows which are parallel to the equatorial plane. By the proper design of temperature conditions, the first-order mode can be made to predominate. This mode is the only mode with flow through the center of the drop. In practice, the convection may be composed of several modes. However, if axisymmetric conditions can be realized, the rate of flow is expected to tend toward an extremum at the axis of the drop. If even-order modes are negligible, an extremum also is to be expected at the center of the drop. A significant even-order component is evidenced if the trajectories of particles across the equatorial plane are not perpendicular to the plane.

\section{Experimental Apparatus}

\section{Buoyancy Convection Apparatus}

Fig. 1 shows a sample bulb, heater and heat exchanger used in the convection experiment and indicates the positions of the heater and of the heat exchanger. The spherical portion of the bulb has a volume of $62.7 \mathrm{~cm}^{3}$. The lower stem of the bulb admits a thermocouple to the lower pole of the bulb. A plastic tube connects this stem to a reservoir of the liquid for the study. The 
upper stem admits a thermocouple to the upper pole of the bulb. Three other thermocouples (not shown) are located about the outside of the bulb: one below, one above and one that is movable.

The heater consists of a coil of Kanthal wire wound in a second helix inside a short length of alumina tube. The wire is held in place by a castable alumina cement. The heater is powered by a filament power supply and, at the maximum output voltage ( $8 \mathrm{~V})$, draws about 2.1 A of current.

The inner wall of the heat exchanger is a copper tube which fits around the upper glass stem of the sample bulb. The outer wall is a plastic tube. The space between these two tubes is loosely packed with copper ribbon to improve the heat transfer to the circulating water.

Fig. 2 shows the apparatus assembled for bright-field illumination: the light beam from the microscope lamp is passing through the box containing the sample bulb and is incident upon the camera. Darkfield illumination dan be obtained by moving the camera to a port on the right-hand side of the box so that the camera views the bulb perpendicular to the incident beam of light. The port for dark-field illumination is not shown in the picture.

The box shields the sample bulb from stray light and reduces the temperature fluctuations near the bulb. The inside of the box is painted with matte black paint to remove the light scattered inside the box. The box consists of two compartments: the upper, and larger, compartment houses the sample bulb and the heat exchanger; the lower compartment houses the heater. The two 
compartments are separated by a floor of corrugated paper backed by a layer of asbestos felt and a thin sheet of molybdenum. A small hole at the center of the floor allows the lower stem of the sample bulb to pass into the lower chamber. A coll of copper tubing circulates water around the perimeter of the heater compartment.

A single-lens reflex camera with a lens focusable down to $0.24 \mathrm{~m}$ (9 $1 / 2$ inches) and panatomic-X film are used to record the flow patterns. To improve detection of the particles in the liquid, the camera can be equipped with a bellows.

Glycerol was used in the preliminary study because it was readily available and its properties were sufficiently well known. Aluminum dust was used as the tracer.

\section{Neutral Buoyancy Cell}

Fig. 3 shows the neutral buoyancy cell. It is fabricated from $9.5 \mathrm{~mm}$ thick acrylic plastic sheet and is $25 \mathrm{~cm}$ high. One pair of opposite walls have ports for feedthroughs. One feedthrough is shown in the picture. This feedthrough contains a teflon core with a hole which matches the outer diameter of the syringe needle and a rubber septum. The teflon core and the rubber septum can be replaced with cores with holes up to $9.5 \mathrm{~mm}$ diameter so that larger tubes can be inserted into the neutral buoyancy cell. Experimental Results

At a power setting of $9.7 \mathrm{~W}(6.0 \mathrm{~V}$ output; $1.62 \mathrm{~A}$ through the heater) glycerol convects slowly. At this power setting, a temperature difference of $101.8^{\circ} \mathrm{C}\left(21.6^{\circ}\right.$, lower pole; $123.4^{\circ}$, upper pole $)$ was measured. For glycerol this temperature difference across the bulb yields a Rayleigh number, $\mathrm{Ra}_{2}^{\prime}$, of $1.03 \times 10^{5}$. 
Fig. 4 is a photograph of particle trajectories in glycerol with a temperature difference of $168^{\circ}$ across the bulb. The particles were photographed using the bellows to give a reproduction ratio of $1: 1$ and with dark-field illumination. The exposure time was 20 s. Conclusions and Discussion

These preliminary results with glycerol show first that buoyancy convection can be established in this viscous liquid and second that the observation of the convection is not obscured by the spherical lens effect. If the Grashof number for the observed buoyancy convection in glycerol is equated to the ratio $\mathrm{N}_{\mathrm{Ma}} / \mathrm{N}_{\mathrm{Pr}}$ for thermocapillary convection in Krytox $143 \mathrm{AA}$ oil, the temperature gradient of $168^{\circ}$ used for the glycerol implies a temperature difference of $34^{\circ}$ for a drop of Krytox oil with the same radius $(2.46 \mathrm{~cm})$.

The best photographs were obtained using dark-field illumination and the bellows. When bright-field illumination was used, the illumination could be improved somewhat if the incident light were oblique to the optic axis of the bulb and camera rather than along the axis so that the condensing effect of the bulb was reduced.

In the present work, the exposures lasted on the order of 5 to 30 s so that they could be timed with a stopwatch. However, if motionpictures are taken, such as with a DAC (data acquisition camera), then timing marks must be recorded on the film or a clock must be included in the objective field of the camera. 


\section{Nomenclature}

$$
\begin{aligned}
& \text { g = magnitude of the gravitational acceleration } \\
& \mathrm{N}_{\mathrm{Gr}}=\mathrm{R}^{3} \rho^{2} \mathrm{~g} \beta\left(\mathrm{T}-\mathrm{T}_{\mathrm{o}}\right) / \mu^{2} \text {, Grashof number } \\
& N_{M a}=\left.\sigma_{T} \nabla T\right|_{R} R^{3} / \mu \alpha \text {, Marangoni number } \\
& \mathrm{N}_{\operatorname{Pr}}=\mu / \rho \alpha \text {, Prandt } 1 \text { number } \\
& \mathrm{R} \quad=\text { radius of bulb or of drop } \\
& R a_{2}^{\prime}=R^{3} \rho g \beta\left(T-T_{0}\right) / \mu \alpha \text {, Rayleigh number } \\
& \mathrm{T}=\text { local temperature } \\
& \mathrm{T}_{\mathrm{o}} \quad=\text { average temperature } \\
& \alpha=\text { thermal diffusivity } \\
& \text { B = bulk thermal expansion coefficient } \\
& \mu \quad=\quad \text { viscosity } \\
& \nu \quad=\mu / \rho \text {, kinematic viscosity } \\
& \rho \quad=\text { density } \\
& \sigma \quad=\text { surface tension } \\
& \sigma_{T}=(\mathrm{d} \sigma / \mathrm{dT})_{\mathrm{T}_{\mathrm{o}}}
\end{aligned}
$$

Acknowledgments: Thanks are due to Mr. Arnold Driver for his assistance with photographing the convection in glycerol. 
References

1. Chandrasekar, S., Hydrodynamic and Hydromagnetic Stability, Oxford University, Clarendon Press, London (1961).

2. Dragoo, A. L., "Steady Thermocapillary Convection Cells in Liquid Drops," to be published: cf. NBS Space Processing Research NBSIR 74-611, E. Passag1ia and R. L. Parker, Eds. (1974).

3. Kintner, R. C., Horton, T. J., Grauman, R. E., and Ambeckar, S., Can. J. Chem. Eng., 39, 235 (1961). 



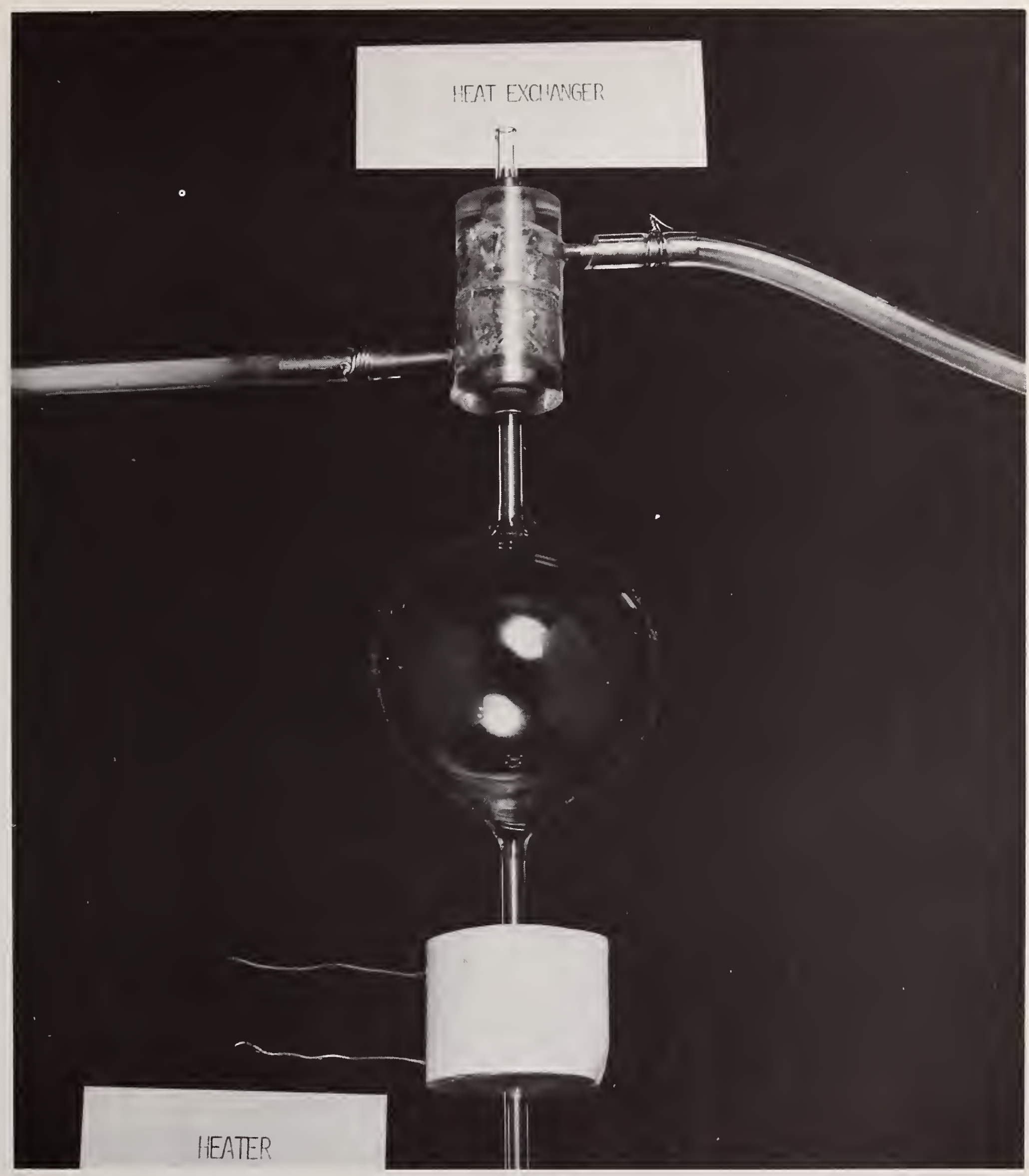

Fig. 1 -- Sample bulb, heater and heat exchanger. 



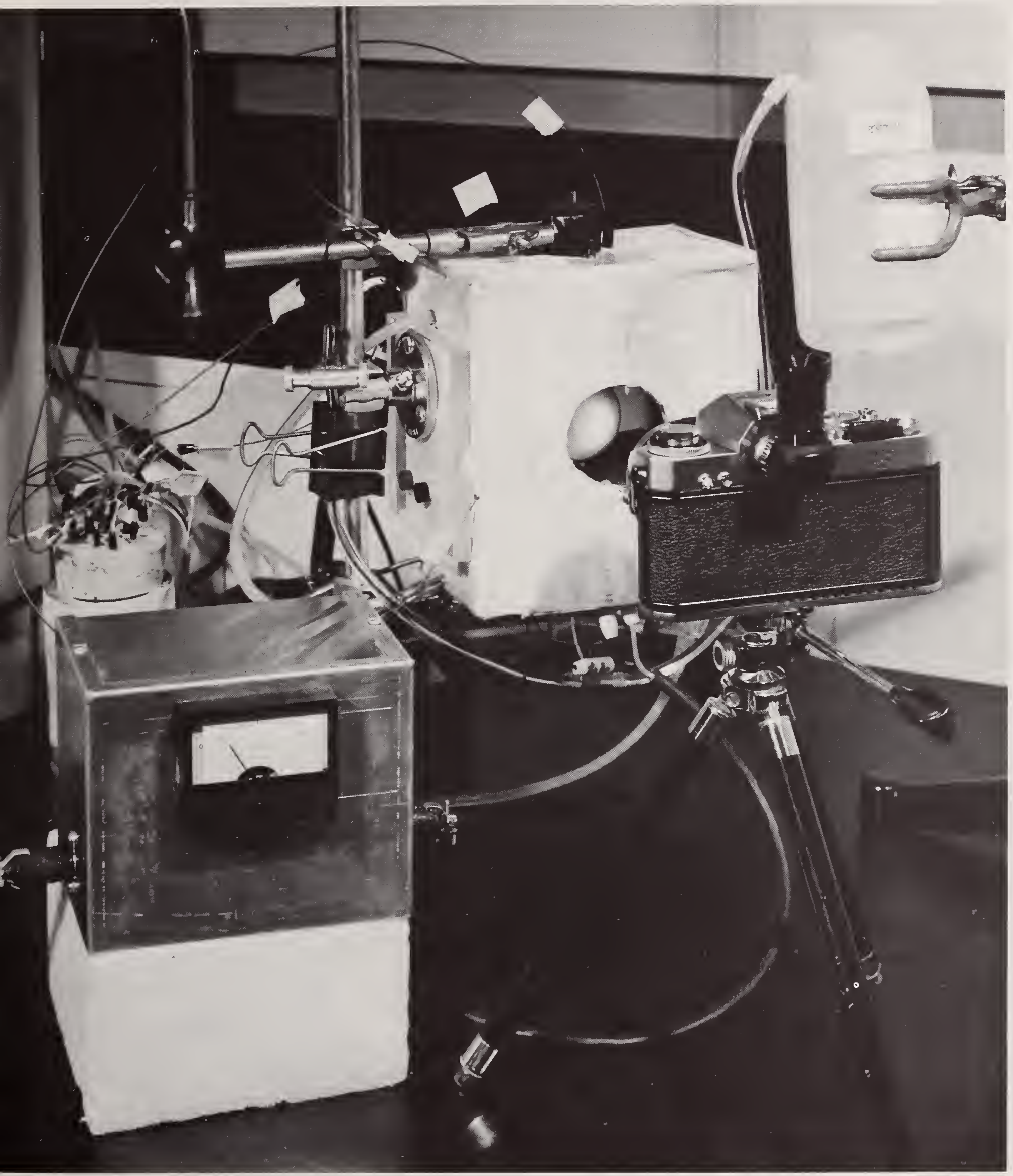

Fig. 2 -- Buoyancy convection apparatus arranged for bright-field illumination. 



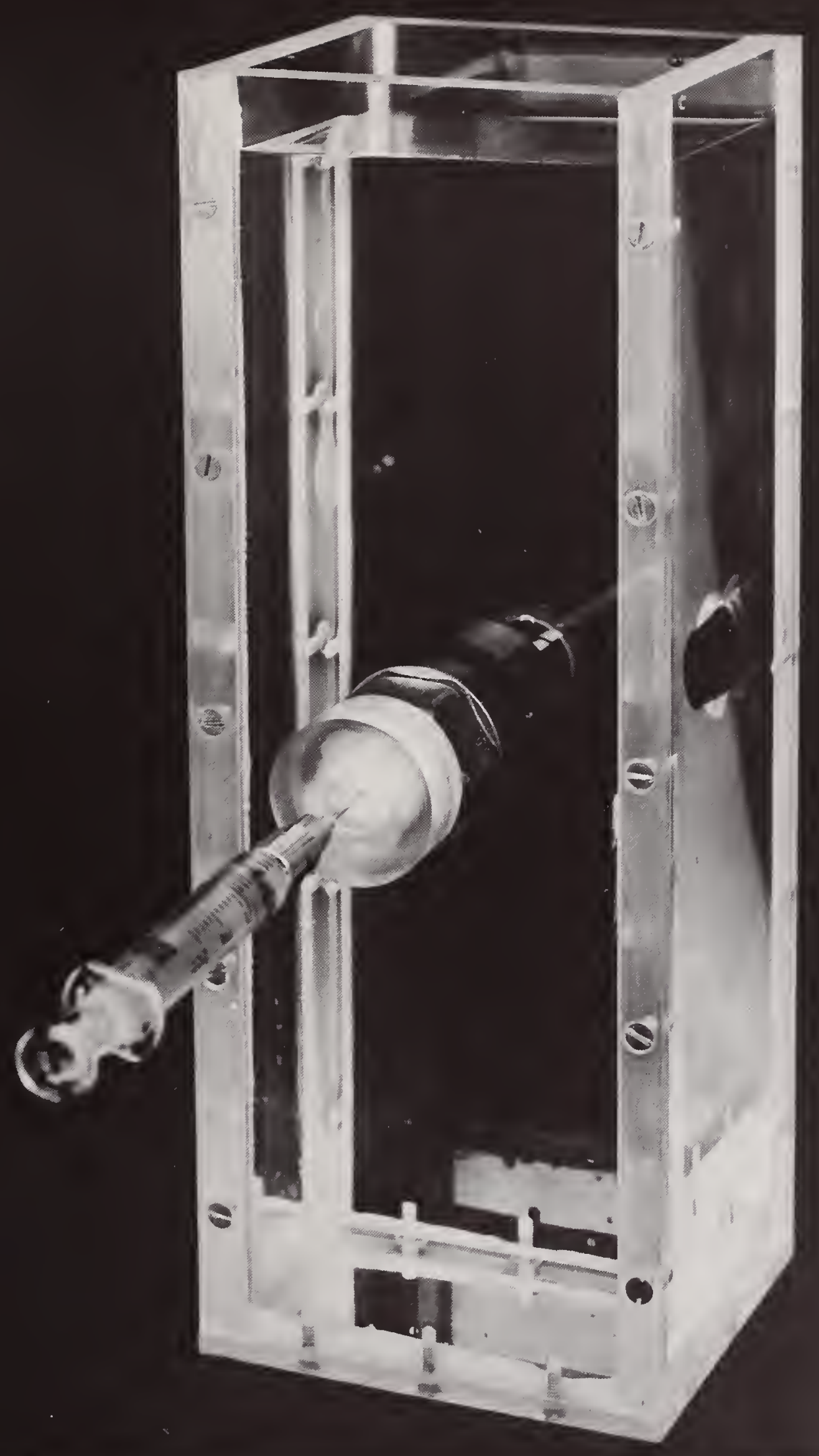

Fig. 3 -- Neutral buoyancy cell. 



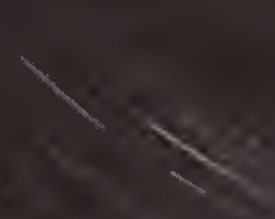

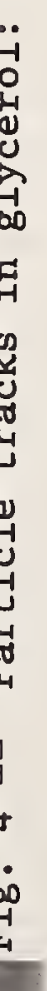





\section{Task 7}

\section{Consulting Support}

NBS personnel continue to consult extensively with NASA Headquarters and NASA field offices as required. We have visited Marshall Space Flight Center, Alabama on numerous occasions and have provided advice on many aspects of the Space Processing Program, including Shuttle Payloads. We continue our Space Processing Seminar at NBS. 

National Aeronautics and Space Administration Washington, D. C. 20546

Mr. F. L. Williams

Code ES

Dr. J. H. Bredt
Code ES
1 copy

4 copies

National Aeronautics and Space Administration Johnson Space Center

Houston, Texas 77058

Mr. W. E. Rice

Mr. J. P. Loftus

Mr. J. A. Mason

Mr. E. J. Svreek
Code EA

Code AT

Code DA

Code FM5
2 copies

1 copy

1 copy

1 copy

National Aeronzutics and Space Administration George C. Marshall Space Flight Center

Marshall Space Flight Center, Alabama 35812

\author{
Mr. H. P. Gierow \\ Mr. K. R. Taylor \\ Mr. B. 0. Montgomery \\ Dr. W. G. Johnson \\ Mr. R. E. Lake \\ $\mathrm{Mr}$. R. Schwinghamer \\ Dr. R. S. Snyder \\ Mr. E. C. McKannan \\ Mr. R. C. Ruff \\ Miss M. H. Johnston \\ Mr. H. Wuenscher \\ Mr. I. C. Yates, Jr. \\ Mr. L. H. Berge \\ Mr. G. M. Arnett \\ Mr. T. C. Bannister \\ Mr. M. C. Davidson \\ Mr. A. C. Krupnick \\ Mr. W. B. McPherson \\ Mr. J. H. Hess \\ Mr. L. L. Lacy \\ Mr. M. F. Nowakowski \\ Dr. R. E. Allen \\ Mr . P. H. Rhodes \\ Mr. J. Bond \\ Mrs. B. R. Facemire \\ Mr. C. F. Schafer \\ Mr. B. R. Aldrich \\ Mr. A. Boese \\ Mr. R. A. Taylor \\ Mr. J. R. Williams \\ Mr. V. H. Yost \\ Dr. M. P. L. Siebel
}

1 copy

2 copies

1 copy

1 copy

1 copy

1 copy

1 copy

1 copy

1 copy

1 copy

1 copy

1 copy

1 copy

1 copy

1 copy

1 copy

1 copy

1 copy

1 copy

1 copy

1 copy

1 copy

1 copy

1 copy

1 copy

1 copy

1 copy

1 copy

1 copy

1 copy

1 copy

1 copy 
Jet Propulsion Laboratory

California Institute of Technology

4800 Oak Grove Drive

Pasadena, California 91103

Dr. C. H. Savage

Code 158-235

1 copy

Dr. M. M. Saffren

Code 183-301

1 copy

Dr. T. G. Wang

Code 183-401

1 copy

Dr. D. D. Elleman

Code 183-401

1 copy

Dr. J. W. Lucas

Code 180-700

1 copy

National Aeronautics and Space Administration Langley Research Center

Hampton, Virginia 23665

Dr. L. T. Melfi, Jr.

Code 401A

Mr. B. W. Cocke, Jr.

Code $401 \mathrm{~A}$

Dr. R. A. Outlaw

Code 234

Dr. J. P. Mugler

Code 215B

Code 215B

Mr. W. C. Ayers
1 copy

1 copy

1 copy

1 copy

1 copy

1 copy

National Aeronautics and Space Administration

Ames Research Center

Moffett Field, California 91103

Dr. J. A. Parker

Code SC

1 copy

Grumman Aerospace Corporation

Bethpage, New York 11714

Dr. C. H. Li

1 copy

General Electric Company

Space Sciences Laboratory

P.0. Box 8555

Philadelphia, Pennsylvania 19101

Dr. R. T. Frost

1 copy

European Space Research Organization

114 Avenue Charles de Gaulle

92 Neuilly, France

Dr. G. Seibert

1 copy

Gesellschaft fur Weltraumforschung mbH

505 Por z-Wahn

Linder Hohe

German Federal Republic

Dr. A. Bewersdorff

1 copy 
NBS.114A (REV. 7.73 )

\begin{tabular}{|c|c|c|}
\hline $\begin{array}{l}\text { U.S. DEPT. OF COMM. } \\
\text { BIBLIOGRAPHIC DATA } \\
\text { SHEET }\end{array}$ & $\begin{array}{l}\text { 1. PUBL.ICATION OR REPORT NO. } \\
\text { NBSIR 76-980 }\end{array}$ & $\begin{array}{l}\text { 2. Gov't Accession } \\
\text { No. }\end{array}$ \\
\hline $\begin{array}{l}\text { 4. TITLE AND SUBTITIE } \\
\text { NBS Space } P r\end{array}$ & \multicolumn{2}{|c|}{ NBS Space Processing Research } \\
\hline \multicolumn{3}{|c|}{$\begin{array}{l}\text { 7. AUTHOR(S) } \\
\text { E. Passaglia, R. L. Parker }\end{array}$} \\
\hline \multicolumn{3}{|c|}{$\begin{array}{l}\text { 9. PERFORMING ORGANIZATION NAME AND ADDRESS } \\
\qquad \begin{array}{l}\text { NATIONAL BUREAU OF STANDARDS } \\
\text { DEPARTMENT OF COMMERCE } \\
\text { WASHINGTON, D.C. } 20234\end{array}\end{array}$} \\
\hline $\begin{array}{l}\text { 12. Spunsoring Organization } \\
\text { NASA - Advanced } \\
600 \text { Independence } \\
\text { Washington, D.C. }\end{array}$ & $\begin{array}{l}\text { and Complete Address (Street, City } \\
\text { ssions Program } \\
\text { venue S.W. } \\
20546\end{array}$ & $t e, Z I P)$ \\
\hline
\end{tabular}

15. SUPPI,EMENTARY NOTIS

Annual Report

16. ABSTRACT (A 200-word or less factual summary of most significant information. If document includes a significant bibliography or literature survey, mention it here.)

This report describes NBS work for NASA in support of NASA's Space Processing Program covering the period November 1, 1974 to December 31, 1975. The objectives of the NBS program are to perform ground-based studies (and, where appropriate, space-based studies) of those aspects of space that cculd possibly provide a unique environment for making materials more perfect or more pure. The approach taken deals primarily with experimental and theoretical studies of the possible effects of the absence of gravitational forces on those materials preparation processes where the presence of gravity may be important in reducing perfection or purity. The materials preparation processes studied comprise six tasks in the areas of crystal growth, purification and chemical processing, and the preparation of composites.

17. KEY WORDS (six to twelve entries; alphabetical order; capitalize only the first letter of the first key word unless a proper name; separated by semicolons)

Convection; crystal growth; crystal perfection; microgravity; purification; space processing.

18. AVAILABILITY $\quad$ Un Unimited

$[\neg$ For Official Distribution. Do Not Release to NTIS

Order From Sup. of Doc., U.S. Government Printing Office Washington, D.C. 20402, SD Cat. No.C13

X Order From National Technical Information Service (NTIS) Springfield, Virginia 22151

\begin{tabular}{|c|c|}
\hline $\begin{array}{c}\text { 19. SECURITY CLASS } \\
\text { (THIS REPURT) } \\
\text { Unclassified } \\
\text { UNCLASSIFIED }\end{array}$ & 21. NO. OF PAGES \\
\hline $\begin{array}{c}\text { 20. SECURITY CLASS } \\
\text { (THIS PAGE) } \\
\text { UNCLASSIFIED }\end{array}$ & 22. Price \\
$\$ 7.75$ \\
\hline
\end{tabular}


